\title{
Photonuclear Data Index
}

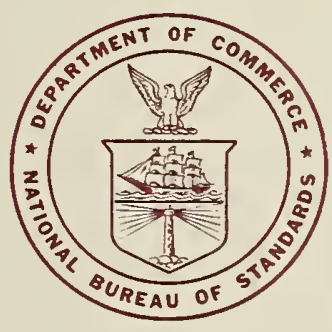

United States Department of Commerce

National Bureau of Standards

Miscellaneous Publication 277 


\section{THE NATIONAL BUREAU OF STANDARDS}

The National Bureau of Standards is a principal focal point in the Federal Government for assuring maximum application of the physical and engineering sciences to the advancement of technology in industry and commerce. Its responsibilities include development and maintenance of the national standards of measurement, and the provisions of means for making measurements consistent with those standards; determination of physical constants and properties of materials; development of methods for testing materials, mechanisms, and structures, and making such tests as may be necessary, particularly for government agencies; cooperation in the establishment of standard practices for incorporation in codes and specifications; advisory service to government agencies on scientific and technical problems; invention and development of devices to serve special needs of the Government; assistance to industry, business, and consumers in the development and acceptance of commercial standards and simplified trade practice recommendations; administration of programs in cooperation with United States business groups and standards organizations for the development of international standards of practice; and maintenance of a clearinghouse for the collection and dissemination of scientific, technical, and engineering information. The scope of the Bureau's activities is suggested in the following listing of its three Institutes and their organizational units.

Institute for Basic Standards. Applied Mathematics. Electricity. Metrology. Mechanics. Heat. Atomic Physics. Physical Chemistry. Laboratory Astrophysics. ${ }^{*}$ Radiation Physics. Radio Standards Laboratory:* Radio Standards Physics; Radio Standards Engineering. Office of Standard Reference Data.

Institute for Materials Research. Analytical Chemistry. Polymers. Metallurgy. Inorganic Materials. Reactor Radiations. Cryogenics. ${ }^{*}$ Materials Evaluation Laboratory. Office of Standard Reference Materials.

Institute for Applied Technology. Building Research. Information Technology. Performance Test Development. Electronic Instrumentation. Textile and Apparel Technology Center. Technical Analysis. Office of Weights and Measures. Office of Engineering Standards. Office of Invention and Innovation. Office of Technical Resources. Clearinghouse for Federal Scientific and Technical Information."

\footnotetext{
* Located at Boulder, Colorado, 80301.

**Located at 5285 Port Royal Road, Springfield, Virginia, 22171.
} 
UNITED STATES DEPARTMENT OF COMMERCE - John T. Connor, Secretary NATIONAL BUREAU OF STANDARDS • A. V. Astin, Director

\title{
Photonuclear Data Index
}

\author{
Prepared by \\ Photonuclear Data Group \\ Institute for Basic Standards \\ National Bureau of Standards \\ Wrashington, D. C.
}

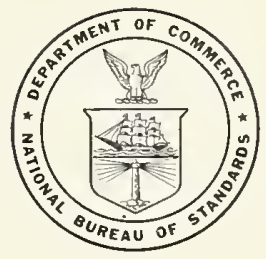

National Bureau of Standards Miscellaneous Publication 277

Issued April 1, 1966 


\section{Photonuclear Data Group}

N. V. Baggett, 'T. M. Collins, E. G. Fuller,

J. C. Holland, J. H. Hubbell, and J. S. O'Connell 


\section{Foreword}

A project on photonuclear data is being conducted in the Radiation Physics Division of the NBS Institute for Basic Standards with the support of the NBS Office of Standard Reference Data. The objective of this project is the creation and maintenance of a current Photonuclear Data File which will be used as source material for a series of miscellaneous reports covering various aspects of the field.

The present publication is the first report of the series. It is essentially an index of the data in the File as of January 1, 1965 . It is expected that it will be possible to make revisions and additions to the index rather rapidly so that it can be kept both complete and up to date. It is anticipated that many of the future publications of the project will contain critically evaluated data and will be included in the National Standard Reference Data Series.

The NBS Office of Standard Reference Data was established to carry out the Bureau's assigned responsibility to administer the National Standard Reference Data System (NSRDS). This System is a governmentwide effort to give the American technical community optimum access to the quantitative data of physical science, critically evaluated and compiled for convenience. The NSRDS was established by the President's Office of Science and Technology, acting upon the recommendation of the Federal Council for Science and Technology. Its general objective is to coordinate and integrate existing data evaluation and compilation activities into a systematic, comprehensive program, supplementing and expanding technical coverage when necessary, establishing and maintaining standards for the output of the participating groups, and providing mechanisms for the dissemination of the output as required.

A. V. Astin, Director. 


\section{Contents}

$\begin{array}{llc}\text { Page } & \text { Pag }\end{array}$

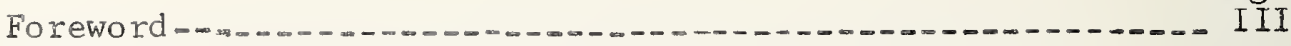

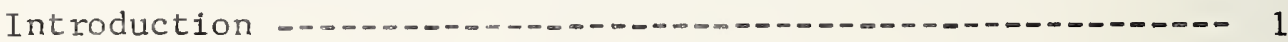

The Photonuclear Data File.......... 3

Scope of the Data Index-..... 3

Description of the Index

I. Reference number (REF)

II. Nucleus excited (NUCLIDE)

III. Reaction -

IV. Type of information (RES) $\ldots \ldots \ldots \ldots$

V. Excitation energy range (EXCIT) _.............. 6

VI. Source type and energy range (SOURCE)

VII. Detector type, energy, and angular range (DETECTOR)

VIII. Remarks -...... 7

References-_.

Data Index _.......... 9

Bibliography - 


\author{
Photonuclear Data Project \\ Radiation Physics Division \\ National Bureau of Standards, Washington, D. C.
}

AB STRACT

An index to experimental data on photonuclear reactions is presented. Organized by element and isotope, each entry in the index supplies quantitative information for a specific reaction on the ranges of excitation energy, source energy, detected particle energy, and emission angles for reaction produced covered in each reference. Information is also given on the type of measurement and detector used.

\title{
INTRODUCTION
}

The function of this data index is to furnish a means of readily locating experimental data in the field of photonuclear reactions. This index differs from a bibliography in that it supplies quantitative information about the content of a paper. Each entry ir the index corresponds to the measurement of a specific photonuclear reaction for a specific nuclide or group of nuclides. An attempt is made to give as complete a description of each measurement as is possible in a single line. The type of measurement is indicated as well as the range of excitation energies covered, the type of detector used and its energy response, and the type of angular distribution data obtained. The object has been to give a description of each measurement that is complete enough to permit an individual looking for specific types of data in the field to locate the pertinent references without having to go through a large number of irrelevant papers as might be the case if only the title of the paper or the reaction studied were listed for each entry.

For the purposes of this index the general criterion as to what constitutes a measurement of photonuclear data is that the measurement must give information on the electromagnetic matrix element between the ground state and excited states of some nucleus. The most common type of reactions are: $(Y, Y),(Y, n),(Y, p),\left(p, Y_{0}\right)$, and $\left(e, e^{1}\right)$. Two reactions which fit the matrix element criterion, but which were not included in the compilation because of their rather special nature are heavy particle coulomb excitation and the thermal neutron capture reaction: $\left(n, Y_{p}\right)$. The energy region of interest is from 0 to about $150 \mathrm{MeV}$. Most of the experiments are concerned with the excitation energy range 8 to $30 \mathrm{MeV}$, the region of the giant dipole resonance.

* Participants in the photonuclear data project: N. V. Baggett, T. M. Collins, E. G. Fuller, J. C. Holland, J. H. Hubbell, and J. S. O'Conne11. 
REF.

R.R. Harvey, J.T. Caldwe 1, R.L. Bramblett, S.C. Fultz

Phys. Rev. 136, B126-31 (1964)

Positron annihilation; ion chamber

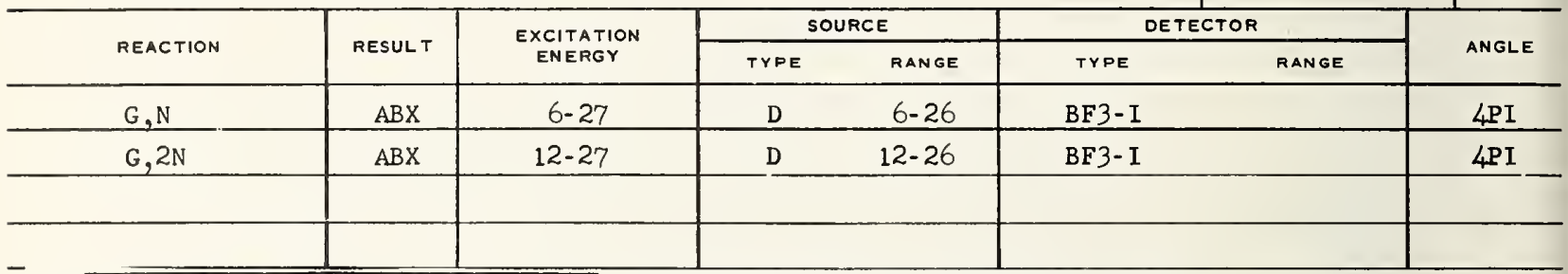

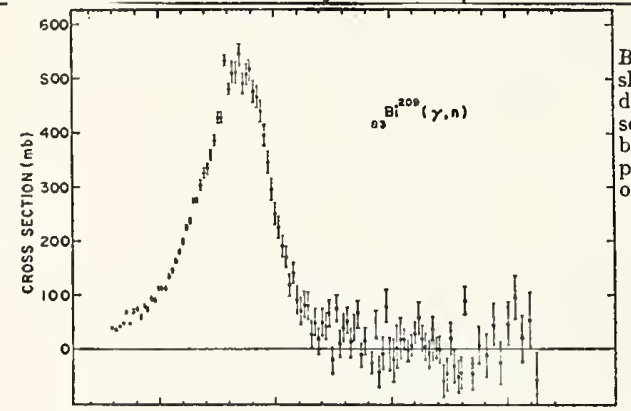

FIG. 4. Top figure shows data points for $\sigma[(\gamma, n)+(\gamma, n p)]$ for $\mathrm{Bi}^{2009}$, obtained from single-neutron counting data. Center figure shows data for $\sigma(\gamma, 2 n)$ obtained from double-neutron counting data. Data points for the compound nucleus formation cross section of $\mathrm{Bi}^{209}$, i.e., $\sigma[(\gamma, n)+(\gamma, n p)]+\sigma(\gamma, 2 n)$ are shown in the bottom figure. Solid curve is a plot of a Lorentz line having the parameters given in Table II. The data are uncertain below $8 \mathrm{MeV}$ wing to low beam intensities encountered.

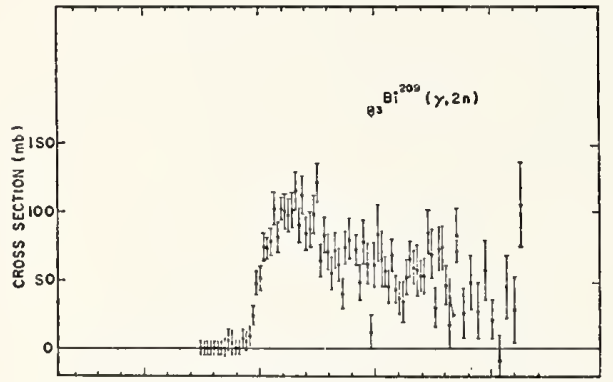

TABLE II. Lorentz line parameters and $\sigma_{-2}$ values for $\mathrm{Pb}$ isotopes and $\mathrm{Bi}$.

\begin{tabular}{|c|c|c|c|c|c|}
\hline Isotope & $\begin{array}{c}\text { Peak } \\
\sigma_{0} \\
(\mathrm{mb}) \\
\end{array}$ & $\begin{array}{c}\text { Width } \\
\Gamma \\
(\mathrm{MeV})\end{array}$ & $\begin{array}{c}\operatorname{lin} \\
(\mathrm{MeV})\end{array}$ & $\left(\mathrm{m}^{\circ}\right)^{\sigma-2} \mathrm{MeV}^{-2}$ & $\begin{array}{l}0.00225 A^{6 / 3} \\
\left(\mathrm{ml}^{6} / \mathrm{MeV}\right)\end{array}$ \\
\hline $\begin{array}{l}\mathrm{Pb}^{206} \\
\mathrm{~Pb}^{207} \\
\mathrm{~Pb}^{2018} \\
\mathrm{Bi}^{200}\end{array}$ & $\begin{array}{l}525 \\
485 \\
495 \\
520\end{array}$ & $\begin{array}{l}3.75 \\
3.87 \\
3.78 \\
3.83\end{array}$ & $\begin{array}{l}13.7 \\
13.6 \\
13.6 \\
13.5\end{array}$ & $\begin{array}{l}15.6 \pm 1.6 \\
14.5 \pm 1.5 \\
14.1 \pm 1.4 \\
16.6 \pm 1.7\end{array}$ & $\begin{array}{l}16.2 \\
16.3 \\
16.4 \\
16.6\end{array}$ \\
\hline
\end{tabular}

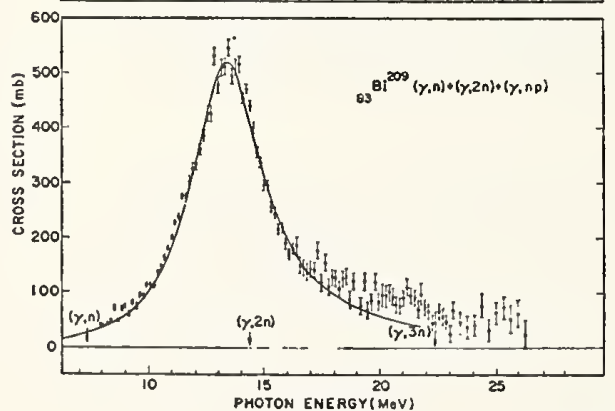

TABLE I. Integrated cross sections in $\mathrm{MeV}-\mathrm{b}$, up to $28 \mathrm{MeV}$, for $\mathrm{Pb}$ isotopes and $\mathrm{Bi}$.

\begin{tabular}{|c|c|c|c|c|c|}
\hline Isotope & $\int_{0}^{28} \sigma(\gamma, n) d l i$ & $\int_{0}^{28} \sigma(\gamma, 2 n) d E$ & $\int_{0}^{28} \sigma d E$ & $\int_{0}^{2 B} \sigma d E+W$ & $0.06 \mathrm{NZ} / \mathrm{A}$ \\
\hline $\begin{array}{l}\mathrm{Pb}^{206} \\
\mathrm{~Pb}^{207} \\
\mathrm{~Pb}^{206} \\
\mathrm{Bi}^{200}\end{array}$ & $\begin{array}{l}2.22 \\
2.05 \\
1.96 \\
2.17\end{array}$ & $\begin{array}{l}0.56 \\
0.60 \\
0.95 \\
0.76\end{array}$ & $\begin{array}{l}2.7 S \pm 0.28 \\
2.65 \pm 0.27 \\
2.91 \pm 0.29 \\
2.93 \pm 0.29\end{array}$ & $\begin{array}{l}3.07 \pm 0.36 \\
2.95 \pm 0.30 \\
3.21 \pm 0.32 \\
3.25 \pm 0.33\end{array}$ & $\begin{array}{l}2.96 \\
2.97 \\
2.98 \\
3.00\end{array}$ \\
\hline
\end{tabular}

Fig. 1. Example of Photoruclear Data Sheet. See text for defiritions of symbols used. 
The source of the data used to compile this index is the Photonuclear Data File. This file consists of data sheets containing abstracts of the significant data from each reference. The data are presented in a form such that an experienced worker in the field of photonuclear reactions can judge the quality of the data contained in each reference. Each sheet gives information about the type of reaction studied and the method of measurement. In addition, the significant figures and tables in the original paper are given, often on a reduced scale. Each data sheet covers either one element or one isotope of an element so that a given reference normally results in several data sheets. Fig. 1 is an example of one of these data sheets.

In addition, a master "reprint" file of each paper abstracted is maintained. Various index files permit the location of a given paper in the compilation by means of any of the authors of the paper or by the institutions at which the work was carried out.

\section{SCOPE UF THE DATA INDEX}

This version of the index covers experimental photonuclear physics articles published in the period from 1955 through 1964. For the period 1955 through 1959 papers were located by using the bibliographies of Toms ${ }^{1}$ and the International Atomic Energy Agency Bibliographical Series No. 10, (Photonuclear Reactions) ${ }^{2}$ and Atomic Energy Review ${ }^{3}$. For the period 1960 through 1964, six journals (Physical Review, Physical Review Letters, Nuclear Physics, Physics Letters, Journal of Experimental and Theoretical Physics (JETP), and Il Nuovo Cimento) were searched - issue by issue - and Nuclear Science Abstracts was used to find papers in other journals. The above-mentioned bibliographies were also used to cross check this period.

Only articles published in journals were abstracted. In a few cases, conference reports were used if the article was fairly complete and $i t$ was likely that this was the final report of the experiment.

\section{DESCRIPTION OF THE INDEX}

The data index of each element begins with the isotopic abundances for that element and a list of particle thresholds for each isotope. The abundances were taken from a compilation by Gladys Fuller. This reference should be consulted for remarks concerning the accuracy of these values and possible variations with the source of the sample. The reaction thresholds were taken from a list of Q-values computed by J. H. E. Mattauch, W. Thiele, and A. H. Wapstra ${ }^{5}$. The values given in reference 5 have been arbitrarily rounded off to the nearest $0.1 \mathrm{MeV}$ except for those cases where the uncertainties quoted are of the order of $1 \mathrm{MeV}$. In these cases, thresholds are given to the nearest $\mathrm{MeV}$. 
In the index itself the data content of an experimental pape is listed under eight categories:

I. Reference number

II. Nucleus excited

III. Reaction

IV. Type of information

V. Excitation energy range

VI. Source type and energy range

VII. Detector type, energy and angular rarge

VIII. Remarks

For each element or isotope if known, entries are grouped by the reactions studied. The entries under a given nucleus and reaction are ordered chronologically.

\section{Reference number (REF)}

The reference number is used to cite the full bibliographical reference which is giver in the bibliography following the data index. This number is made up of the year and the first two letters of the first author's name, plus an additional serial number. For example, a paper published in 1963 by Galileo would have a reference number 63 Gal.

\section{Nucleus excited (NUCLIDE)}

The atomic number ( $\mathrm{Z}$ ), chemical symbol, and mass number ( $A$ ) of the excited nucleus (not necessarily the target nucleus) is given. The mass number is 1 isted only if the isotopic assignment is unambiguous.

\section{Reaction}

The following table defines the symbols used under IN, OUT:

$\$$ polarization of the incident or outgoing particle or polarization or alignment of the target

A alpha particle

D deuteron

E electron 


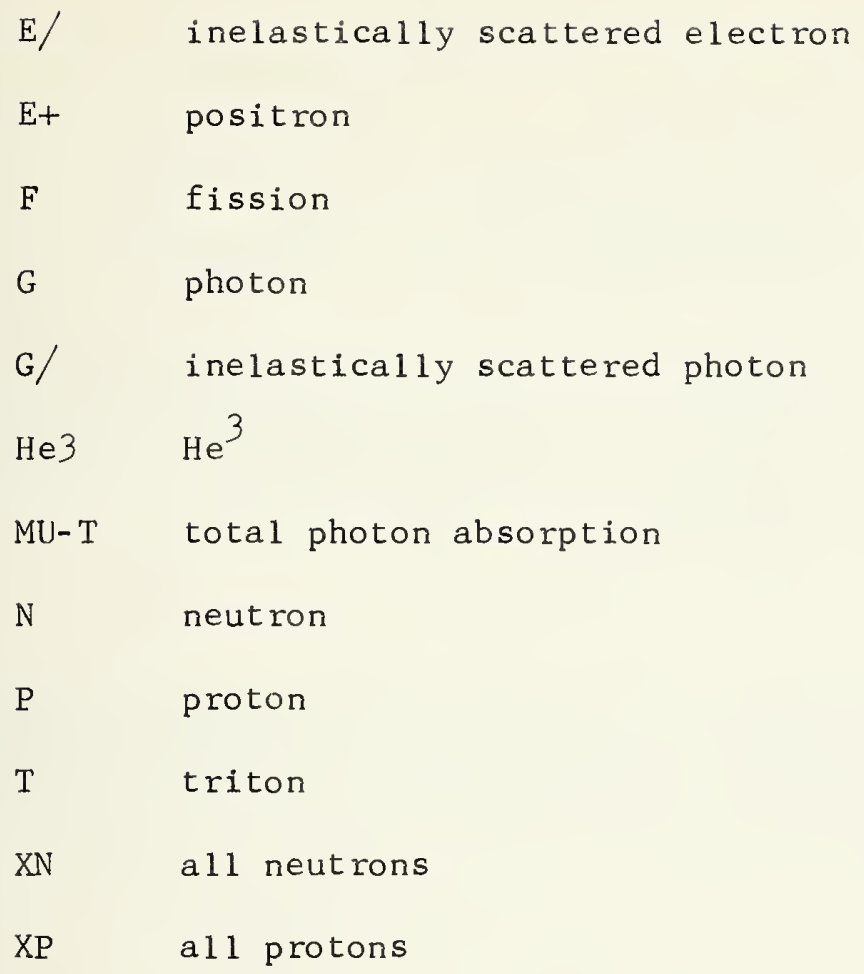

When two reaction products are listed under OUT they were detected in coincidence, e.g., the notation $E, E / P$ means the inelastically scattered electron and proton were detected in coincidence in a reaction of the type: $A+e \rightarrow(A-1)+e+p$, while $E$, $P$ means that only the proton was detected.

IV. Type of information (RES)

The following catalog of abbreviations applies to the type of result obtained in an experiment:

$A B I$ absolute integrated cross-section data $\int \delta d E_{Y}$

ABX absolute cross-section data

ABY absolute yield data

FMF form factor

LFT excitated state lifetime

NOX no cross-section data

RLI relative integrated cross-section data 
V. Excitation energy range (EXCIT)

The excitation energy range of the nucleus involved in the gammaray transition is given in $\mathrm{MeV}$. For reactions initiated by gamma rays the excitation energy is taken as the gamma-ray energy; for reactions initiated by particles the binding energy and kinematic corrections are made. The abbreviation THR stands for threshold.

VI. Source type and energy range (SOURCE)

The source of incident particles is characterized by the letter $C$ or $D$ indicating that the source was continuous or discrete in energy. The source energy is indicated under MIN-MAX. The usual source of photons is bremsstrahlung which would be marked $C$. The range of end point energies is given.

VII. Detector type, energy, and angular range (DETECTOR)

The following abbreviations apply under detector TYPE:

ACT measurement of radioactivity of the target

BBL bubble chamber

BF3 $\mathrm{BF}_{3}$ neutron counter with moderator, e.g., Halpern detector, long counter

$\mathrm{CCH} \quad$ cloud chamber

EMU emulsions (photographic plates)

IфN $\quad$ ionization chamber

MAG magnetic spectrometer

MGC magnetic Compton spectrometer

MGP magnetic pair spectrometer

MळD moderated neutron detector not employing a $\mathrm{BF}_{3}$ counter, e.g., rhodium foil, Szilard-Chalmers reaction, $\mathrm{He}^{3}, \mathrm{Li}^{6}$ reactions 


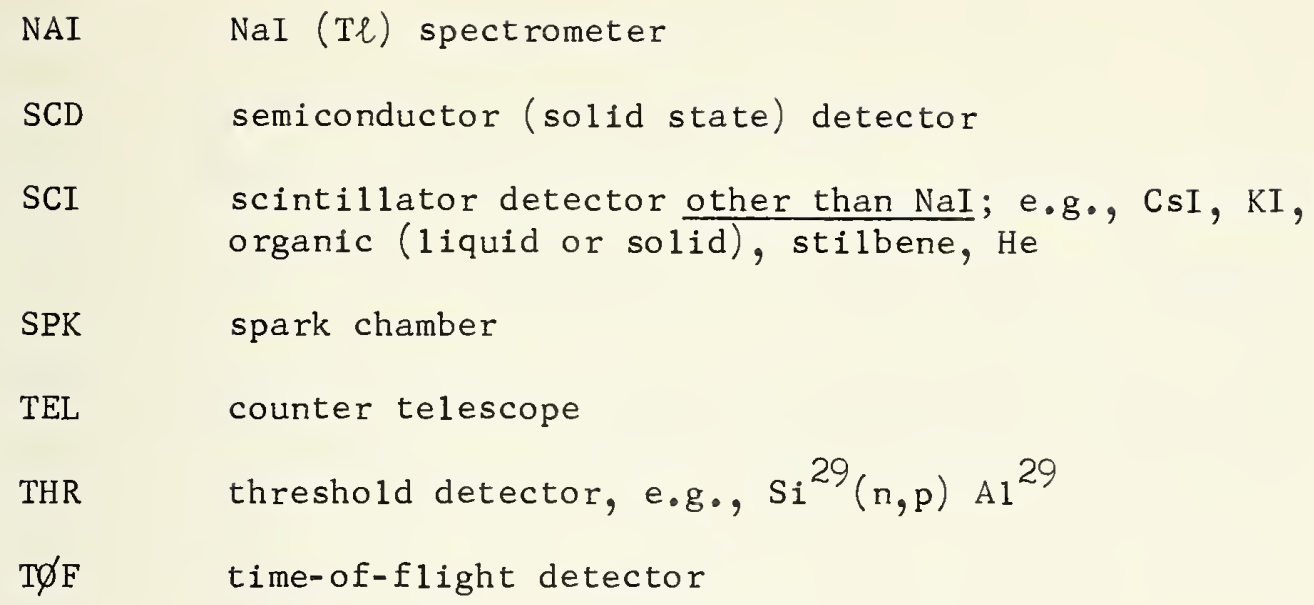

The symbols D or I under TYPE means that the reaction product was detected differentially or integrally in energy. For example, a scintillator (SCI) is usually used differentially (D) while a $B_{3}$ detector used with a neutron moderator (BF3) integrates over neutron energy. The range of detected particle angular distribution is shown under ANG with the following designations:
$0-180$
one number in this column means the measurements were made at this angle only (angle in degrees)
$4 \mathrm{PI}$
a $4 \pi$ geometry was used or a method like radioactivity or a total absorption measurement
DST
an angular distribution was measured

VIII. Remarks

This additional information was selected in a fairly unsystematic way and limited by the available space. It should therefore not be regarded as exhaustive or consistent. An asterisk indicates that a completed data sheet was not in the file as of the time the bibliographic card was prepared. In this case the information given in the index was obtained from the abstract of the article referenced.

Some of the abbreviations used in this column are:

BREAKS levels located by "breaks" in the yield curve
$\mathrm{CF} \quad$ compared with
COINC coincidence
DT BAL detail balance


G-WIDTH $\Gamma_{\gamma}$, gamma-ray transition width

J-PI spin and parity assignments of levels are made

POL polarization

Q-SQUARE momentum transfer squared $\left(q^{2}\right)$

REL relative

SEP ISOTPS separated isotope used

\section{REFERENCES}

1. M. E. Toms, "Bibliography of Photo- and Electronuclear Disintegrations", Bibliography No.22, U.S. Naval Research Laboratory, Washington, D. C.

2. Bibliographical Series No.10, "Photonuclear Reactions", International Atomic Energy Agency, Vienna.

3. B. I. Goryachev, Atomic Energy Review 2, 71-148 (1964).

4. Gladys Fuller, 1959 Nuclear Data Tables (NUCLEAR DATA PROJECT)National Academy of Sciences, National Research Council, Washington, D。C.

5. J.H. E. Mattauch, W. Thiele, and A. H. Wapstra, Nuclear Physics 67, $32(1965)$. 


\begin{tabular}{|c|c|c|c|c|c|c|c|c|c|}
\hline A & ABUND. & & SE & I ION & ENERGIES & (MEV) & & & \\
\hline & & $G, N$ & $G, P$ & $G, T$ & $\mathrm{G}, \mathrm{HE} 3$ & $G, A$ & $G, 2 \mathrm{~N}$ & $G, N P$ & $G, 2 P$ \\
\hline 1 & 99.99 & $*$ & * & * & * & * & * & * & * \\
\hline 2 & $1.5(-2)$ & 2.2 & 2.2 & * & * & * & $*$ & * & * \\
\hline 3 & * & 6.3 & $8 \cdot 5$ & * & * & * & 8.5 & $8 \cdot 5$ & * \\
\hline
\end{tabular}

REF

$58 \mathrm{MO} 2$

$60 \mathrm{WY} 1$

$580 \times 1$

$58 \mathrm{AL} 1$

$550 \times 1$

$60 \mathrm{GO} 1$

$613 A 3$

63ST 1

59FR 1

$61 K E 3$

$62 \mathrm{BA} 1$

62PE 1

$63 \mathrm{BA} 1$

$57 B A 1$

$58 \mathrm{BA} 1$

6OGE 3

$61 \mathrm{JOI}$

6ITAl

$63 \mathrm{BE} 7$

$63 \mathrm{BO} 4$

$63 \mathrm{FRI}$

55001

56001

$55 \mathrm{ALI}$

56D I I

$56 \mathrm{KE} 1$

$56 \mathrm{WHI}$

$57 \mathrm{ALI}$

$58 T A 2$

$58 \mathrm{WHI}$

6OFE2

60GA

$61 \mathrm{MY} 1$

63LOI

64L I I

$64 \mathrm{BO} 2$

$64 \mathrm{IM}$
NUCLIDE REACTION RES $Z$ A IN,OUT

$\begin{array}{llll}I H & 1 & G, M U-T & A B X \\ 1 H & 1 & G, M U-T & A B X \\ 1 H & 1 & G, G & A B X \\ 1 H & 1 & G, G & A B X \\ 1 H & 1 & G, G & A B X \\ 1 H & 1 & G, G & A B X \\ 1 H & 1 & G, G & A B X \\ 1 H & 1 & G, G & A B X\end{array}$

EXCIT

SOURCE

MIN-MAX TYPE MIN-MAX

C 94

C 90

C 87

SCI - D
NAI-D 13-80
TEL-D

$30-95$
75

C 75

C 247

C 900
IH 2 E,E/ SPC

IH 2 E,E/ RLX

$1 \mathrm{H} 2$

$1 \mathrm{H} 2$

$1 \mathrm{H} 2$

$1 \mathrm{H} 2$

$1 \mathrm{H} 2$

$1 \mathrm{H} 2$

$1 \mathrm{H} 2$

IH 2

IH 2

$1 \mathrm{H} 2$

$1 \mathrm{H} 2$

$1 \mathrm{H} 2$

$1 \mathrm{H} 2$

$1 \mathrm{H} 2$

$1 \mathrm{H} 2$

$1 \mathrm{H} 2$

$1 \mathrm{H} 2$

IH 2

IH 2

IH 2

IH 2

IH 2

IH 2

$1 \mathrm{H} 2$

$1 \mathrm{H} 2$

1 H 3

$1 \mathrm{H} 4$

$E, E /$

$E, E /$

$G, N$

$G, N$

$G, N$

$\$ G, N$

$G, N$

$\Phi G, N$

$\Phi G, N$

$\Phi G, N$

$G, N P$

$G, N P$

$G, P$

$G, P$

$G, P$

$G, P$

$G, P$

$G, P$

$G, P$

$\$ G, P$

$G, P$

$G, P$

$\Phi G, P$

$\Phi G, P$

$G, N$

$N, G$

$A B X$

$A B X$

RLX

NOX
$E, E /$

$0-95$
$0-20$
0

D175

D204-500

MAG-D

D 42

D 42

$M A G-D$

O 20

$\begin{array}{ll}D & 42 \\ D & 42\end{array}$

$\begin{array}{lll}\text { RLY } & \text { THR } & 265 \\ A B X & \text { THR } & 255\end{array}$

$\begin{array}{ll}A B X & T H R \\ \text { NOX } & \text { THR }\end{array}$

C -265

$M A G-D$

$M A G-D$

C170,255

CTHR

NOX 01

D 3

C 22

NOX $1230 \quad 30$

$\mathrm{SPC} \quad 3 \quad 9$

1123

c $3-9$

C 24

c

RLX THR $340 \quad C 340$

$A B X \quad 20 \quad 65 \quad C \quad 20-65$ EMU-I

ABX THR 293 C136-293 TEL-D

$A B X \quad 75 \quad 450 \quad C 150-450$ TEL-D

$A B X \quad 60250$ C $60-250$ EMU-D

ABX THR 148 C 54-148 TEL-D

$A B X$ THR $200 \quad C 200$

$A B X \quad 923 \quad C \quad 9-23 \quad N A I-I$

$A B X 150300 \quad C 150-300$ EMU-D

$A B X \quad 5090 \quad C 94 \quad$ TEL-D

$A B X 350900 \quad C 500-900$ TEL-D

NOX 2294 C294 SPK-D

RLX $80-140$ C400-500 MAG-D

DST
$124 *$
DST *
DST
DST *
90

4P I *

DST $Q=206 \mathrm{MEV} / \mathrm{C}$

145

180 GAS TARGET

180 SOLID TARGET

180

DST *

DST

4PI THRESHOLD

DST NEUTRONS POLARIZED

DST

DST NEUTRONS POLARIZED

DST NEUTRONS POLARIZED

148 NEUTRONS POLARIZED

DST *

DST *

DST

DST *

DST

DST

DST *

DST *

DST

44 PROTONS POLARIZED

DST

DST

58

90 PHOTONS POLARIZED

4P I

4PI NO H4 FOUND 
A ABUND. (1)

$3 \quad 1.4(-4) \quad 7.7$

$4 \quad 99.99 \quad 20.6$
SEPARATION ENERGIES (MEV)

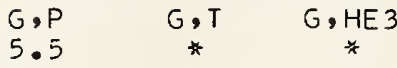

$19.8 \quad 19.8 \quad 20.6$

(1) ABUNDANCE DEPENDS ON SOURCE

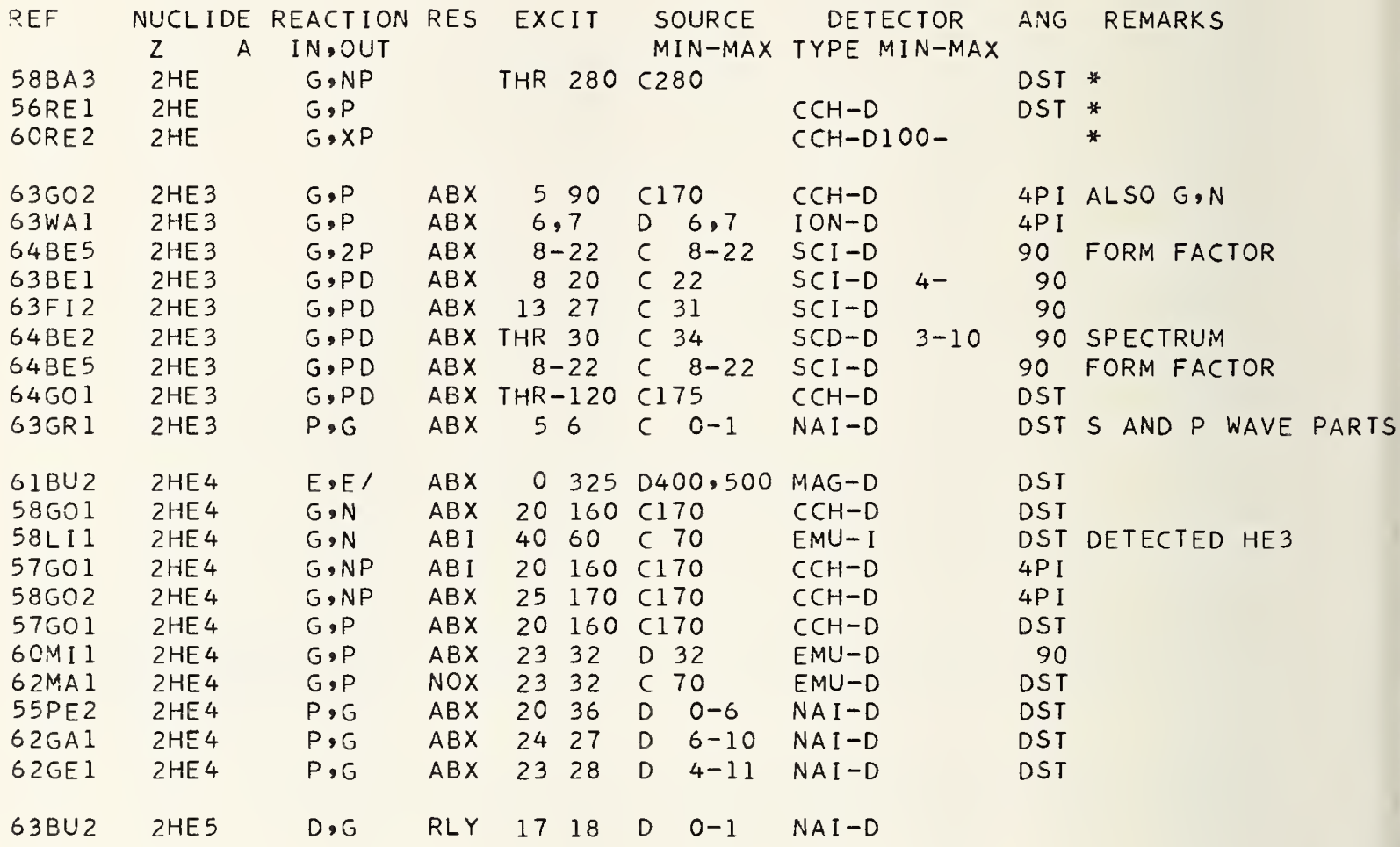

\section{LITHIUM $\quad Z=3$}

\begin{tabular}{|c|c|c|c|c|c|c|c|c|c|}
\hline A & ABUND. 11$)$ & & SE & ATION & ENERGIES & (MEV) & & & \\
\hline 6 & 7.42 & $G, N$ & $\begin{array}{l}G, P \\
4.7\end{array}$ & $\begin{array}{r}G, T \\
15,8\end{array}$ & $\begin{array}{l}\text { G, HE } 3 \\
15.8\end{array}$ & $\begin{array}{l}G, A \\
1,5\end{array}$ & $G, \underset{t}{2 N}$ & $\begin{array}{r}G, N P \\
3.7\end{array}$ & $G \cdot 2$ \\
\hline 7 & 92.58 & 7.3 & 10.0 & 2.5 & 28.3 & 2.5 & 12.9 & 12.0 & \\
\hline
\end{tabular}

(1) ABUNDANCE DEPENDS ON SOURCE

\begin{tabular}{|c|c|c|c|c|c|c|c|c|c|c|}
\hline REF & NUCL IDE & REACT ION & RES & EXCI T & SOURCE & DET & ECTOR & ANG & REMARKS & \\
\hline & Z A & IN,OUT & & & MIN-MAX & & MI N-MAX & & & \\
\hline $58 \mathrm{AL} 1$ & $3 \mathrm{LI}$ & $G, G$ & $A B X$ & 2 & & & & 124 & * & \\
\hline $64 G 03$ & $3 \mathrm{LI}$ & $E, E /$ & $A B X$ & 4 & D $40-70$ & $M A G-D$ & & 180 & FMF & \\
\hline $60 \mathrm{KU} 2$ & $3 \mathrm{LI}$ & $G, N$ & $A B X$ & THR 90 & C 90 & TEL -D & $10-$ & DST & & \\
\hline 55BA 5 & $3 \mathrm{LI}$ & $G, \times N$ & $A B X$ & THR -250 & $C 250$ & & $30-$ & DST & * & \\
\hline $55 \mathrm{HE} 1$ & $3 \mathrm{LI}$ & $G, \times N$ & RLX & 1332 & C 13-32 & BF 3-I & & 4PI & ENR I CHED & L I 7 \\
\hline 58RY 1 & $3 \mathrm{LI}$ & $G, \times N$ & $A B X$ & 524 & $5-24$ & $B F 3-I$ & & 4PI & & \\
\hline 60FAl & $3 \mathrm{LI}$ & $G, \times N$ & $A B X$ & 1156 & C 11-57 & BF 3-I & & 4P I & & \\
\hline 63003 & $3 \mathrm{LI}$ & $G, \times N$ & $A B X$ & 875 & $8-100$ & BF 3-I & & 4PI & & \\
\hline 64AL 1 & $3 \mathrm{LI}$ & $G, \times N$ & $A B X$ & THR 19 & -19 & $B F 3-I$ & & 4P I & & \\
\hline 55001 & $3 \mathrm{LI}$ & $G, N P$ & & THR -252 & $C 140-252$ & $S C I-D$ & & DST & * & \\
\hline
\end{tabular}


REF

56001

$58 \mathrm{BA} 3$

$56 \mathrm{KLI}$

60KO5

$63 \mathrm{KII}$

$63 K \cup 2$

$58 \mathrm{WH} 2$

6IMA2

62BE 1

$55 \mathrm{MII}$

$61 \mathrm{SH} 6$

$63 K \cup 2$

64 IMI

64ER 2

$59 C O 4$

62806

$625 E 1$

$635 K 1$

60BA 4

$63 \mathrm{BAI}$

$63 B E 2$

$638 E 8$

$63 \mathrm{BII}$

$56 E D 2$

SOPR I

64GR2

$59 R O 1$

$63 \mathrm{COI}$

$64 B A 2$

6OPR I

6OBA3

$60 \mathrm{KO} 3$

6OPR I

$62 \mathrm{VO} 1$

$64 \mathrm{SH} 2$

$60 \mathrm{CHI}$

$62 \mathrm{CH}^{2}$

59DA I

$62 \mathrm{VOI}$

$64 \mathrm{KO} 4$

$60 \mathrm{CHI}$

$62 \mathrm{CH} 2$

$62 \mathrm{VO} I$

$64 \times 04$

$60 \mathrm{CHI}$

64NEI

$58 B E 3$

$595 W 1$

60803

$62 \mathrm{MOI}$

$63 \mathrm{MOI}$

$64 \mathrm{BOI}$

63BA I

$63 B E 3$

$63 B E 8$

$64 \mathrm{BI} 4$

64GR2

$59 R O 1$

6OSTI
NUCLIDE REACTION RES

$Z$ A IN,OUT

$\begin{array}{lllll}3 L I & G, N P & R L X & T H R-340 & C 340 \\ 3 L I & G, N P & & \text { THR } 280 & C 280 \\ \text { 3LI } & \text { G,P } & \text { SPC THR } 80 & C 30\end{array}$

SOURCE

DETECTOR

ANG REMARKS

L1

$3 L I$

$3 L I$

3 LI

$3 L I$

$3 L I$

$3 L I$

$3 L I$

$3 L I$

$3 L I$

3LI 4

3 L I 6

3 LI 6

3 L I 6

$3 L 16$

3L I 6

3 L I 6

3 L I 6

$3 L I 6$

3 L I 6

3 L I 6

3 L I 6

3 LI 6

3 L I 6

$3 L I 6$

3L I 6

$3 L I 6$

3 LI 6

3 LI 6

3 L I 6

3 L I 6

$3 L I 6$

3 LI 6

3 L I 6

$3 L I 6$

$3 L I 6$

3 LI 6

3 L I 6

$3 L I 6$

$3 L$ I 6

3 L I 6

3 L I 6

3 L I 6

3 LI 6

$G, P$

$G, P \quad A B X \quad 1030 \quad C \quad 30$

$G, X P$

$G, X P$

$G, D$

$G, T$

$G, T$

$G, T$

$P, G \quad A B Y$

$A B X \quad 40100 C 45-110$.

NOX $1021 \quad C \quad 21$

SCI -D $1-10$

RLY THR-2I CTHR-21

THR 10

C 10

$A B X \quad 12 \quad 27$

c 30

EMU-I

D $0-3 \quad N A I-D$

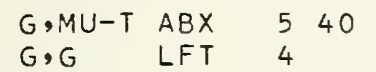

$G, G$

$G, G$

$G, G$

$E, E /$

$E, E /$

$E, E /$

$E, E /$

$E, E /$

LFT

NOX

LFT

$A B I$

$A B I$

FMF

FMF

$G, N$

$A B X$

$G, N$

$A B X$

$G, N$

RL I

GXN ABX

$G, X N \quad A B X$

$G, X N \quad A B X$

$G, N P$ RLI

$G, P$

$G, P$

$G, P$

$G, P$

$G, P$

$G, X P$

$G, X P$

$G, D$

$G, D$

$G, D$

$G, \times D$

$G, \times D$

$G, T$

$G, T$

$G, \times T$

$\mathrm{G}, \mathrm{H}^{4}$

$A B Y$

$S P C$

SPC

$A B Y$

$A B X$

ABX

RLY

$A B X$

$A B Y$

$$
\text { 5-40 }
$$

C 6

C 4

C 17

C 4

D 40

$\begin{array}{llll}3 & 16 & D & 42\end{array}$

28

D101

7 DI00-180 MAG-D

NAI I D

NAI -D

NAI I D

NAI -D

MAG-D

MAG-D

$M A G-D$

540

D101

D 6

$\begin{array}{llll}5 & 17 & C & 17\end{array}$

$M A G-D$

MOD-I

59 D $5-9$

520

550

5-60

$\begin{array}{lll}7 & 17\end{array}$

3080

523

$\begin{array}{ll}5 & 17\end{array}$

1190

$8-10$

C 4-20

$S C I-I$

BF 3-I

$B F 3-I$

$B F 3-I$

c 5-60

C 17

BF 3-I

SCI-I

c $35-87$

C 28

c 17

TEL-D

EMU-D $1-18$

SCI-D 5-9

c $30-90$

c 10

c 90

TEL-D 7-15

EMU-D 1-3

TEL-I 15-30

$20 \quad 90$

C $30-90$

D 2,3

TEL-D 15-30

I $O N-D$

890

C $30-90$

C 35

RLY 1790 C 90

RLY $16 \quad 90$

ABY $23 \quad 90$

SPC THR 35

c 90

C $30-90$

c 35

RLY $3190 \quad$ C 90

ABY $\quad 24-250 \quad C 250$

3 L I 7

$3 L I 7$

3L I 7

$3 \mathrm{LI} 7$

3 L I 7

3L I 7

3LI 7

3L I 7

3L I 7

3 LA 7

3LI 7

3 L I 7

3L I 7
$G, G$

$G, G$

$G, G$

$G, G$

$G, G$

$G, G$

E,E/

$E, E /$

$E, E /$

$E, E /$

$G, N$

$G, X N$

$G, N P$
LFT 1

$\begin{array}{ll}\text { LFT } & 1 \\ \text { LFT } & 1\end{array}$

LFT 0

LFT 0

LFT 0

LFT 13

ABI $\quad 7 \quad 14$ 
Li

$\mathrm{Be}$

\section{$62 G R 2$}

$62 \mathrm{LII}$

$62 \mathrm{SHI}$

$62 \vee 01$

$63 \mathrm{KU1}$

$63 N E 1$

$64 \mathrm{SH} 1$

$60 \mathrm{CHI}$

$62 \mathrm{CH}_{2}$

$62 \mathrm{VOI}$

$60 \mathrm{CH} 1$

$62 \mathrm{CH} 2$

$60 \mathrm{MI} 2$

$62 \mathrm{VOI}$

$63 \mathrm{KUI}$

$60 \mathrm{CHI}$

$64 N E I$
NUCLIDE REACTION RES EXCIT $Z$ A IN, OUT

$3 \mathrm{LI} 7$

$3 \mathrm{LI} 7$

$3 \mathrm{LI} 7$

3 L I 7

3L I 7

3 L I 7

3L I 7

$3 \mathrm{LI} 7$

3 L I 7

3L I 7

3 L I 7

$3 \operatorname{LI} 7$

3 L I 7

$3 \operatorname{LI} 7$

3 LI 7

$3 \operatorname{LI} 7$

3LI 7
$G$,P $A B Y \quad 1030$

$\$ G, P$

$G, P$

$G, P$

$G, P$

$G, 2 P$

$G, 2 P$

$G, X P$

$G, X P$

$G, D$

$G, \times D$

$G, \times D$

$G, T$

$G, T$

$G, T$

$G, X T$

$\mathrm{G}, \mathrm{H}^{4}$
ABY 1030

ABX 1216

$A B Y \quad 1790$

ABY $3 \quad 10$

ABY THR 320

$A B X$ THR-210

ABX 2590

RLY $25 \quad 90$

ABY 1790

RLX $25 \quad 90$

$\begin{array}{lll}R L Y & 25 & 90\end{array}$

$A B X \quad 615$

$A B Y \quad 14 \quad 90$

ABY $14 \quad 25$

RLX 1990

ABY 23-250
SOURCE

MIN-MAX TYPE MIN-MAX

C $10-30 \quad$ ACT-I

C355 TEL-D 155

C $12-16$

EMU-D 2-5

C $25-90$

D 20-30

C 320

c 210

C 90

C 25-90

C 25-90

C 90

C 25-90

c $11-20$

C $25-90$

c 30

C 90

C 250
TEL-D 7-15

TEL-D 4-12

$A C T-I$

ACT - I

TEL-I $15-30$

$T E L \rightarrow D$

TEL-D 7-15

TEL-D 15-31

$T E L-D$

EMU-D

TEL-D 7-15

TEL-D 7-13

TEL-D $17-30$

ACT-I
ANG

REMARKS

4PI

DST PLR P EY C SCT

DST G-WDTH

DST

DST

4P I

90 H5 LIMIT

DST

DST REL TO DEUTERONS

DST

DST REL TO PROTONS, SPC

DST REL TO PROTONS

DST J-PI

DST

DST

DST REL TO PROTONS, SPC

4PI NO H4 FOUND

BERYLLIUM $Z=4$

A ABUND。

$9 \quad 100.00$
SEPARATION ENERGIES (MEV)

$\begin{array}{rrrrrr}G, N & G, P & G, T & G, H E 3 & G, A & G, 2 N \\ 1.7 & 16.9 & 17.7 & 21.2 & 2.5 & 20.6\end{array}$

$2.5 \quad 20.6$

$G, N P$

$G, 2 P$

*
REF

$59 \mathrm{GE} I$

6OMA2

$61 M E 1$

$62 \mathrm{CA} 3$

$63 \mathrm{MII}$

63PE 1

63REI

63RII

$64 \mathrm{SC2}$

64TAI

$62 \mathrm{MI} 4$

64 TE 1

60BA 4

$62 E D I$

$63 N G 1$

$63 N G 2$

55101

5SED 1

56FAI

$53 A S$ I.

$58 \mathrm{BA} 2$

$59 \mathrm{GII}$

$59 \mathrm{THI}$

$60 \mathrm{KU2}$

61 JAI

$62 \mathrm{BO} 4$

$62 \mathrm{BO} 7$

62 CUI

62102

$63 \mathrm{BO} 4$

$64 \mathrm{CO} 1$
NUCLIDE REACTION RES Z A IN.OUT

$4 B E 8$

4BE 8

$4 B E 8$

4BE 8

$4 B E 8$

4BE 8

$42 E 8$

4BE 8

4BE 8

$P, G$

$P, G$

$A B X$

EXCIT

SOURCE

DETECTOR

ANG

REMARKS

4BE 9

पBE 9

4BE9

$4 B E 9$

4BE9

4BE9

4BE9

4BE 9

4BE9

पBE 9

4BE 9

4BE 9

4BE9

पBE 9

4BE9

$\triangle B E 9$

4BE 9

4BE 9

4BE 9

$4 B E 9$

4 BE9

$P, G$

2125

$M I N-M A X$

TYPE MIN-MAX

$P, 0$

$P, G$

$P, G$

$P, G$

$P, G$

$P, G$

$P, G$

NOX

1718

D $0-1$

RLX 1820$$
\text { D }
$$

D 0

$N A I-D$

90

DST

DST

DST

DST

DST

DST

$A B X \quad 1926 \quad D \quad 4-8 \quad$ NAI-D

LFT 18 D $0-3$ NAI-D

$\begin{array}{llllll}\text { RLX } & 17 & 19 & D & 0-2 & \text { NAI }-D\end{array}$

$A B X$

2125

D $4-9$

NAI-D

90

DST $J-P I$

GSMU-T ABX $1527 \quad C \quad 31 \quad$ MGC-D $15-32$

G, MU-T ABX 2021 D 20-21 NAI-D

E.E $\quad A B I$ 2

$M A G-D \quad 36-42$

E,E/ SPC 020

$E, E /$

$E, E /$

LFT

D 42

TEL-D 22-42

$G, N$

$G, N$

$G, N$

$G, N$

$G, N$

$G, N$

$G, N$

$G, N$

$G, N$

$\$ G, N$

$G, N$

$G, N$

$G, N$

$\$ G, N$

$\$ G, N$

FMF

16

447

$M A G-D$ 
NUCLIDE REACTION RES EXCIT $Z$ A IN,OUT

$58 B A 2$

$59 B A 3$

$57 \mathrm{LOI}$

$64 A R 2$

$55 B A 5$

$550 I 1$

$59 K \cup 1$

6OBE 1

6 CST 1

$56 \subset 01$

$56 \mathrm{KL} 1$

56 ST 1

$57 \mathrm{CH} 1$

$58 \mathrm{CH} 1$

$62 \mathrm{CL} 1$

$62 C U 1$

$62 \mathrm{LI} 1$

$62 \mathrm{VO} 2$

$63 \mathrm{KI} 1$

$55 \mathrm{TAl}$

58 W'H2

$59 \mathrm{CHI}$

$60 \mathrm{CH} 1$

$62 \mathrm{CH} 2$

$62 B A 1$

$59 \mathrm{CH} 1$

$60 \mathrm{CH} 1$

$62 \mathrm{CH} 2$

$60 \mathrm{CHI}$

$64 \mathrm{BE} 1$

$64 \mathrm{BE} 1$
$E, N \quad A B X$

4BE9 E,N ABI

4BE9

4859

पBE9

पBE9

4BE9

4BE9

$4 \mathrm{BE} 9$

4BE9

$4 \mathrm{~B} 59$

4BE9

4 BE 9

4BE9

4BE 9

$4 B E 9$

$4 B E 9$

4BE 9

पBE9

पBE?

पBE 9

पBE9

4 BE 9

पBE9

पBE9

4BE9

4 BE9

$4 \mathrm{BE} 9$

4 BE9

$4 B E 9$

4BE9
$\mathrm{G}, 2 \mathrm{~N}$

$G, 2 N$

$G, X N$

$G, X N$

$G, X N$

$G, X N$

$G, N P$

$G, P$

$G, P$

$G, P$

$G, P$

$G, P$

$G, P$

$G, P$

$\$ G, P$

$G, P$

$G, P$

$G, X P$

$G, X P$

$G, X P$

$G, X P$

$G, X P$

$G, D$

$G, X D$

$G, X D$

$G, X D$

$G, X T$

$G, A$

$G, 2 A$
SPC
216

ABY $20 \quad 30$

RLY 2157

NOX 270

NOX 1288

$A B X \quad 217$

ABY 1924

780

SPC 1725

SPC 1780

SPC THR 44

$A B X \quad 16 \quad 54$

$A B X \quad 16 \quad 28$

NOX 190

RLY $30 \quad 90$

$A B X \quad 4090$

$A B Y \quad 4060$

FLY 1900

$\begin{array}{lll} & \end{array}$

SPC THR 34
SOURCE

DETECTOR

ANG REMARKS

Be

MIN-MAX TYPE MIN-MAX

C $2-16$ BF $3-1$

$-17$

C 20-30

C 20430 ACT-I

$A B X T H R-250$

c 250

c 70

$A C T-I$

C 88

TEL-I 10-

RLX 149320

C 320

C 24

C 30-80

c 25

C 60-80

C 44

C 16-54

C 32

C 355

$A B X \quad 4090$

C 40-90

NOX THR $335 \quad C 335$

$A B X \quad 67-280 \quad C 280$

$A B X \quad 40100$

1790

C 45

C 90

c 90

C $40-90$

C 45-62

c 90

C 90

C 40-90

C 90

c 34

C 18-34
4PI COMPARED WITH G,N 
REF NUCLIDE

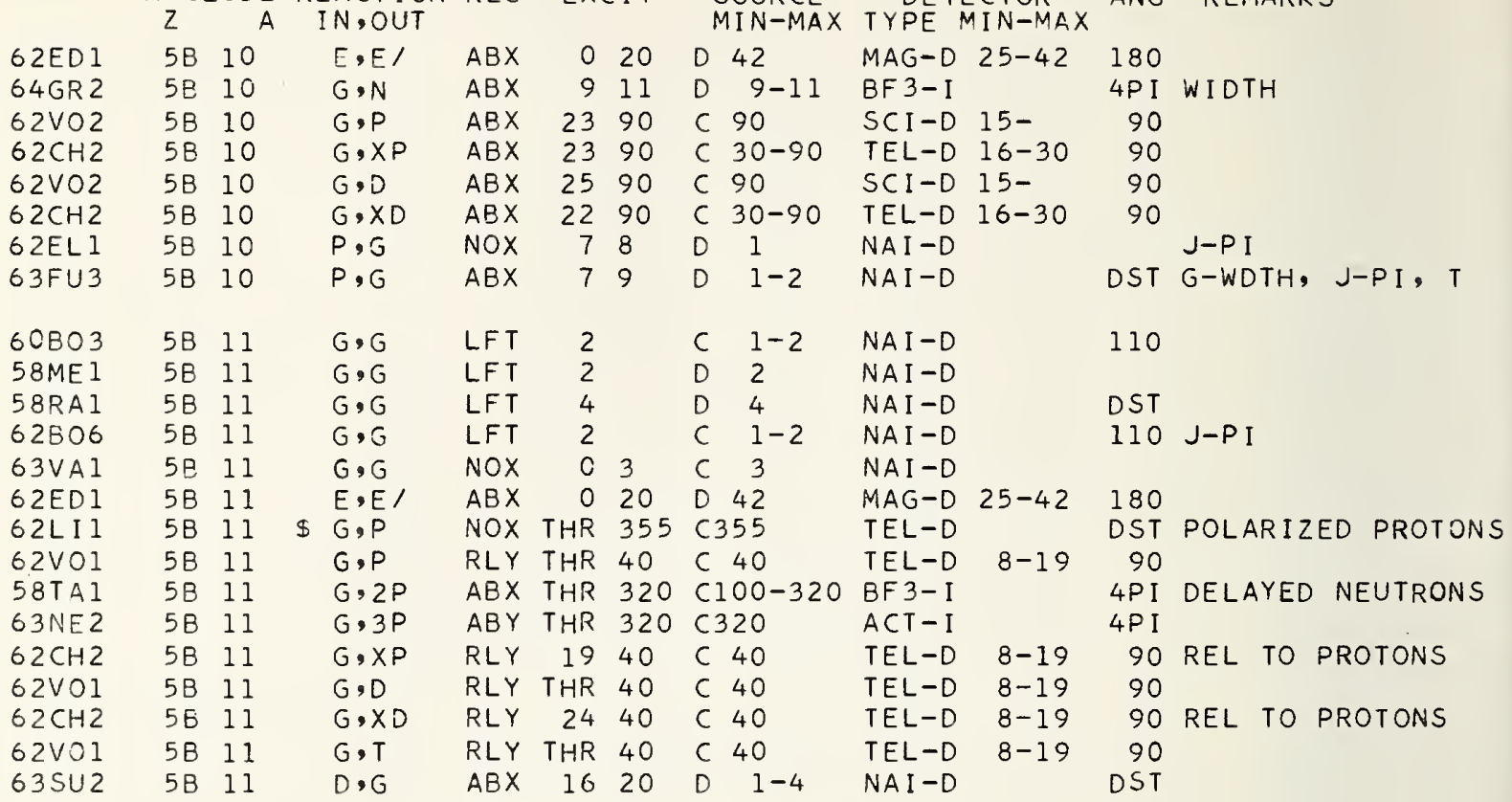

\section{CARBON $Z=6$}

\begin{tabular}{|c|c|c|c|c|c|c|c|c|c|}
\hline A & ABUND.11) & & S & AT I ON & ENERGIES & (MEV) & & & \\
\hline , & 98.89 & $\begin{array}{r}G, N \\
18.7\end{array}$ & $\begin{array}{r}G, P \\
16.0\end{array}$ & $\begin{array}{r}G, T \\
27.4\end{array}$ & G, HE 3 & $\begin{array}{l}G, A \\
7,4\end{array}$ & $G, 2 N$ & G,NP & $G, 2 P$ \\
\hline 3 & 1.11 & 4.9 & 17.5 & 23.9 & 24.4 & 10.7 & 23.7 & 20.9 & 31.6 \\
\hline
\end{tabular}

(1) ABUNDANCE DEPENDS ON SOURCE 
REF

NUCL IDE REACTION RES

2

6IDE4

6 IWI I

62SE 1

$635 C 3$

59PE5

$56 \mathrm{FRI}$

59BAI

60BA4

$61 \mathrm{BO} 4$

GIDUI

62EDI

$63 \mathrm{BO} 3$

$63 \mathrm{GO} 4$

$63 L E I$

$64 \mathrm{CR} 1$

$64 \mathrm{GO} 2$

$64 \mathrm{GO} 3$

64 AMI

$56 \mathrm{TZ2}$

$58 \mathrm{BA} 5$

6OEM2

$62 F U 5$

62MI 2

$558 A 5$

550 I I

$56 \mathrm{FAl}$

$578 A 1$

$58 B A 1$

$59 K U 1$

6 IPR I

$62 \mathrm{MI} 2$

$63 \mathrm{CO} 3$

$63 \mathrm{FUI}$

$58 \mathrm{WH} 2$

$60 \mathrm{STI}$

64TA3

$55 \mathrm{JO} 1$

$55 \mathrm{ST} 2$

$56 \mathrm{KL} \mathrm{I}$

56MAI

$56 \mathrm{STI}$

$57 \mathrm{CHI}$

57 L I I

$59 B A 2$

$64 \mathrm{SH}_{4}$

64TA3

$55 \mathrm{TAl}$

$56 \mathrm{COI}$

$57 M I 2$

$58 \mathrm{BA} 4$

$58 \mathrm{CHI}$

$59 \mathrm{BA} 2$

$59 \mathrm{CHI}$

59PE 4

6OBA I

$60 \mathrm{CHI}$

6ICE I

GIVAI

$62 \mathrm{CH} 2$

$62 \mathrm{HE} \mathrm{I}$

62 LI I

\section{A IN,OUT}

$G, G$

$G, G$

$G, G$

$G, G$

$G, G /$

$E, E /$

$E, E /$

$E, E /$

E,E/

E,E/

$E, E /$

E, E/

E, E/

E, E/

$E, E /$

$E, E /$

E, E/

$E, E / P$

$G, N$

$G, N$

$G, N$

$G, N$

$G, N$

$G, X N$

$G, \times N$

$G, X N$

$G, X N$

$G, X N$

$G, X N$

$G, X N$

$G, \times N$

$G, X N$

$G, X N$

$G, N P$

$G, N P$

$G, N P$

$G, P$

$G, P$

$G, P$

$G, P$

$G, P$

$G, P$

$G, P$

$G, P$

$G, P$

$G, X P$

$G, X P$

$G, X P$

$G, X P$

$G, X P$

$G, X P$

$G, X P$

$G, X P$

$G, X P$

$G, X P$

$G, X P$

$G, X P$

$G, X P$

$G, X P$

$\$ G, X P$
$G, P$

$A B X$

SPC

$\mathrm{ABX}$

$A B X$

$A B X$

$A B X$

EXCIT

SOURCE

MIN-MAX TYPTECTOR ANG REMARKS

C

1936 C 32

NAI $-D$

$50-130$

50

TEL-D

NAI -D

-Max

0
15

$A B X \quad 19-61$

C 19

NAI $-D$

$2-19$

140

DST *

C $19-61$

$0-35$ DI 87

$\begin{array}{lllll}A B X & 0 & 30 & D & 43 \\ S P C & 0 & 30 & D & 42\end{array}$

SPC $\quad 0 \quad 164 \quad 0194$

MAG-D

MAG-D $10-43 \quad 160$

MAG-D 12-42 DST ABI, MULTIPOLARITY

$M A G-D$

13-18 D 46-154 MAG-D

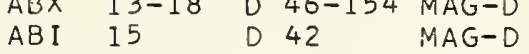

4250200

MAG-D

$20-190 \quad 135$

$030 \quad 042$

035 D 55

$4 \quad 10$

D250

$A B X \quad 10-32$

D $40-70$

15

D $40-70$

RLX THR I50 D550

ABX 22-24 C 22-24

RLX 19-21

19-21

SPC $20 \quad 30$ C 30

$A B X \quad 2131 \quad C \quad 31$

ABX $18 \quad 26 \quad$ D $18-26$

$A B X$ THR-250 C250

NOX THR 70 C 70

NOX THR 23 C 23

RLY THR 265 C -265

ABY THR $255 \quad C 170,255$

ABY THR 88 C 88

RLY $30 \quad 85$

ABX $18 \quad 26$

C $30-8$

$A B X$ THR 80

D $18-26$

CTHR-80

$A B X 20 \quad 29$

C 31

$M A G-D \quad 12-42 \quad 180$

MAG-D 20-55 180 Q-RANGE $0-1$

$M A G-D$

$M A G-D$

$M A G-D$

$M A G-D$

ABX THR 110 C 40-110

RLX $157 \quad 320 \quad C 320$

SPC THR-100 CI70

RLY THR 65 C 65

SPC THR-23

C 23

EMU-D $1-10$ DST

$S C I-D \quad 1-10 \quad 0 S T$

$\mathrm{BF} 3-\mathrm{I}$

$30-$ DST *

$S C I-I \quad D S T$

SCI-I DST

DST WIDTHS

DST FMF

I $80 \mathrm{FMF}$

51

* NEUTRON WIDTH

* BREAKS

SPC THR 80

C $30-80$

ABX 18

D 18

SPC THR 25

C 25

THR-I 21 -

DST *

TEL-D $15-40$ DST

THR-I 1I- 4PI

BF 3-I 4PI

$B F 3-1$

SCI-D 2-11 90

DST COINC WITH BF 3

TEL-D 37-65

76 REL TO H2 CROS SEC

(

$\mathrm{CCH}-\mathrm{D}$

4PI

SCI-D 14- DST

SPC THR 80

C $60-80$

SPC THR-35

C 35

EMU-D

EMU-D

EMU-D $\quad 0-3$

EMU-D

EMU-D

EMU-D

SPC $34 \quad 89$ C 82,89

SCI-D I 8-52

$A B X \quad 20 \quad 23 \quad D \quad 20-23$

SPC THR-100 C 170

$S C I-D$

$\mathrm{CCH}-\mathrm{D}$

$\begin{array}{lll}A B X & -280 & C 280\end{array}$

ABY THR 24 C 24

SPC THR-3I C 31

RLX THR 40

$c$

SPC THR 44

SPC THR 89

$-40 \quad M A G-D$

SPC THR 80

30,44

TEL-D 50-75

*

DST * MULTIPOLARITY

ABX 2262

C 82,89

C 80

ABY THR 90

C 22-90

EMU $-D$

4PI

C 90

ABX THR 342 D245-342

NOX $20 \quad 40$ C 24,40

EMU-D

TEL-D I8-52

SCI -D DST

DST * YIELD

DST *

90

4PI WIDTHS

$4 P I$

RLY THR 90 C 90

SPC $19 \quad 27$ C 31

RLY THR 335 C335

$\begin{array}{rrr}\text { TEL-D } & 19-39 & 58 \\ \text { TEL-I } & 16-30 & \text { DST REL TO DEUTERONS }\end{array}$ 
62 PAI

63F 14

$63 K I 1$

63 MUI

63 WA2

6 IVAI

62001

$58 \mathrm{MA} 1$

56L I 1

$58 M A 1$

$62 \mathrm{MO} 2$

$60 \mathrm{CHI}$

$59 \mathrm{CHI}$

$62 \mathrm{CH} 2$

$55 \mathrm{HA} 1$

$58 \mathrm{MAI}$

60GA2

GISE3

$635 H_{2}$

$61 J A 2$

$620 P 2$

56 T21

$64 \mathrm{GUI}$

55 BA 1

55 SP 2

$57 C A 2$

$58 \mathrm{BA} 2$

59CO 1

59SAI

60GE2

$60 \mathrm{GE} 3$

61 RO 2

$62 \mathrm{BO} 3$

$62 D E 1$

$58 \mathrm{BA} 2$

$590 \mathrm{Cl}$

$57 \mathrm{CO} 1$

$58 B A 2$

61THI

58 TA 1

$55 \mathrm{CA} 2$

55001

$55 \mathrm{GL} 1$

$55 \mathrm{GOI}$

$57 M U 1$

$64 \mathrm{GR} I$

64 TO 1

$59 \mathrm{GE} 1$

$59 \mathrm{GO} 1$

$61 \mathrm{GO} 2$

GISE2

$63 B E 5$

$63 R E 1$

64AL 2

64AL 3

$64 \mathrm{BL} 1$

\section{A IN,OUT}

6C G.XP

6C

$6 C$

6C

6C

$6 C$

$6 \mathrm{C}$

$6 C$

6C

$6 C$

$6 C$

$6 C$

$6 C$

$6 C$

$6 C$

$6 C$

$6 c$

$6 C$

6C

6C $11 \quad P, G$

6C 11

6C 12

6C 12

6C 12

6C 12

6C 12

6C 12

6C 12

6C 12

6C 12

6C 12

6C 12

6C 12

6C 12

6C 12

6C 12

6C 12

6C 12

6C 12

6C 12

6C 12

6C 12

6C 12

6C 12

6C 12

6C 12

6C 12

6C 12

6C 12

6C 12

6C 12

6C 12

6C 12

6C 12

6C 12

6C 12

\section{$G, X P$}

$G, X P$

$G, X P$

$G, X P$

$E, P$

$E, X P$

$G: P T$

$G, P A$

$G, P A$

$G, P A$

$G, X D$

$G, X D$

$G, X D$

$G, 3 A$

$G, 3 A$

$G, 3 A$

$G \cdot 3 A$

$G, 3 A$

$P, G$
$P, G$

$G, N$

$G, N$

$G, N$

$G, N$

$G, N$

$G, N$

$G, N$

$G, N$

$G, N$

$G, N$

$G, N$

$E, N$

$G, 2 N$

$G, X N$

$E, N$

$G, P$

$G, 3 P$

$G, 3 A$

$G, 3 A$

$G, 3 A$

$G, 3 A$

$G .3 A$

$G, 3 A$

$G, 3 A$

$P, G$

$P, G$

$P, G$

$P, G$

$P, G$

$P, G$

$P, G$

$P, G$

HE $3, G$
ABY THR $150 \quad \mathrm{C} 150$

SPC $19 \quad 30 \quad C \quad 31$

ABY THR $335 \quad 335$

ABY THR 24 C 24

RLY THR 65 C 65

NOX 2040

C 24,40

SPC $25 \quad 30$

D 25-30

$A B X$ THR 250 C150,250 EMU-D

$A B X 3050$ C 25-70 EMU-I

$A B X$ THR $250 \quad C 150,250$ EMU-D

$\begin{array}{llll}A B I & 25 & 120 & C 120\end{array}$

EMU-D

RLX THR 90 C 90

SPC THR 80 C 80

RLY THR 90 C 90

TEL-I 16-30

$\begin{array}{lll}R L Y & 9 & 33\end{array}$

C 27.33

TEL-D $15-30$

$A B X$ THR $250 \quad 150.250$ EMU

$A B X \quad 15,18$ D 15,18 EMU-I

RLY 18 D 18 EMU-D

NOX $870<70$

$E M U-D$
$E M U-D$

NOX 10

$A B X \quad 1114$

D $0-2$

NAI - D

NAI I D

\section{G, MU-T ABX 23}

$E, E / F M F \quad 15$
RLX THR

$0^{23}$

C 18-260 ACT-I

ABX $20 \quad 31 \quad C \quad 20-31$ ACT-I

RLY 24145 D 24-145 ACT-I

$A B X \quad 2021 \quad D \quad 20-21 \quad A C T-I$

RLX 19-31 C 31 ACT-I

RLY 1920

c 19.

$A C T-I$
$A C T-I$

NOX THR

CTHR

BF 3-I

$A B X \quad 1923 \quad C \quad 19-23 \quad A C T-I$

$A B \times 1890 \quad C \quad 18-90 \quad A C T-I$

ABX $20 \quad 21 \quad D \quad 20-21 \quad A C T-I$

RLY $24 \quad 145$ D $24-145$ ACT-I

RLI THR-100 CTHR-100 ACT-I

ABX $1941 \quad C \quad 19-40 \quad$ BF 3-I

RLY 24145 D $24-145$ ACT-I

$A B ! 1823$ C $18-23 \quad A C T-I$

$A B \times 100320 \quad(100-320 \quad$ ACT-I

$A B X \quad 12-18$ D $12-18$ EMU-D

$A B X \quad 770 \quad C 70$ EMU-D

SPC 18 D 18 EMU-D

C EMU-D

15.18 D 15,18 EMU-D

$A B Y \quad 724 \quad C \quad 17-24$ EMU-I

$A B X \quad 922 \quad C 22 \quad E M U-D$

$A B X \quad 1923 \quad C \quad 4-8$

$R L \times \quad 1926 \quad 0 \quad 3-11$

ABX $19 \quad 27$

D 3-11

RLY 16

D 0

RLY 2128

D 6-13

$A B X \quad 30 \quad 39$

$A B \times \quad 1929$

D $15-25$

NOX $20 \quad 30$

D $4-14$

$A B \times 28$
NAI -D

NAI -D 19-26

NAI - D

NA I - I

NAI -D

NAI I D

NAI I D

NAI $-D$
NAI - I

60

O SPECTRUM

20

$S T$

DST

2-13 DST

DST * SPC

DST * SPC

4P I

DST REL TO PROTONS

* yld rel to g,

DST REL TO PROTONS

DST

DST * SPC

4PI

4PI Q-VALUE DISTRIB

90 J-PI, G-WIDTH
0

DST $Q=0.2-0.5$, WIDTH

4PI ABY 18-250

* threshold

4PI

4PI COMPARED WITH $E, N$

4PI

4PI * BREAKS

4PI THRESHOLD

4PI THRESHOLD

4PI

4P I

4PI 
REF

60ED 1

$64 G R 2$

$57 \mathrm{COI}$

$615 A 2$

$57 \mathrm{CO}$

$64 \mathrm{KO} 2$

64DE2
NUCLIDE REACTION RES $Z$ A IN,OUT $6 C 13$

6C 13

$6 C \quad 13$

6C 13

6C 13

6C 13

\section{$G, N$}

$$
G, N
$$

$A B X$ $G, \times N$ $G, \times N$ $G, P$ $G, P$ $G, \times P$

$$
A B X
$$

$A B X$

$S P C$

$A B X$

$S P C$

$A B X$
EXCIT

67

$\begin{array}{ll}6 & 41\end{array}$

$\begin{array}{ll}7 & 14\end{array}$

$17 \quad 45$

$20-32$

$18-50$
SOURCE MIN-MAX TYPE MIN-MAX

ANG REMARKS

D 6-7

BF 3-I
$B F 3-I$

$B F 3-I$

TOF $-D$

D $6-11$

C 14

C 17-40 ACT-I

c 32

C $18-50$
4P I

$4 P I$

$4 \mathrm{PI}$

79

4 PI

ACT -I 4 PI

NI TROGEN $Z=7$

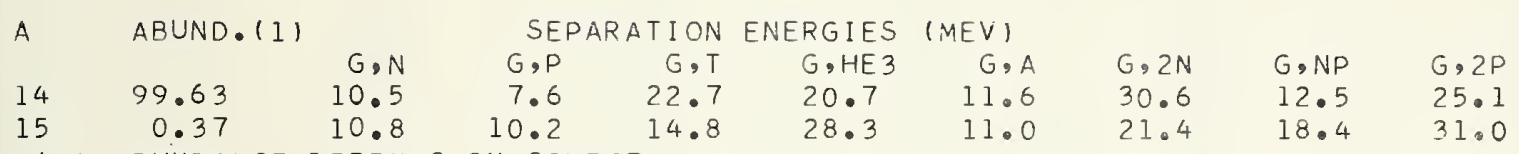

(1) ABUNDANCE DEPENDS ON SOURCE

REF NUCLIDE REACTION RES EXCIT SOURCE DETECTOR ANG REMARKS

\section{Z A IN,OUT}

$64 \mathrm{BO} \quad 7 \mathrm{~N}$

6OMU1 $7 \mathrm{~N}$

60BA7 $7 \mathrm{~N}$

$63 F \cup 2 \quad 7 N$

$60 \mathrm{BA} 7 \quad 7 \mathrm{~N}$

$60 K O 1 \quad 7 N$

$56 \mathrm{JOI} \quad 7 \mathrm{~N}$

$57 L I I \quad 7 N$

$586 R 1 \quad 7 \mathrm{~N}$

6OBA7 $\quad 7 \mathrm{~N}$

$60 K O 1 \quad 7 \mathrm{~N}$

6OWAI $7 \mathrm{~N}$

6ORE2 $7 \mathrm{~N}$

63 FI $4 \quad 7 N$

$64 \mathrm{KOI} \quad 7 \mathrm{~N}$

$58 \mathrm{MAI} \quad 7 \mathrm{~N}$

$62 M O 2 \quad 7 N$

$64 \mathrm{TO2} \quad 7 \mathrm{~N}$

$60 H E 2 \quad 7 N \quad 13$

62PA2 $7 \mathrm{~N} 13$

62WAI $7 \mathrm{~N} 13$

$63 \mathrm{FII} \quad 7 \mathrm{~N} 13$

$63 Y 02 \quad 7 N \quad 13$

60CAI $7 \mathrm{~N} 14$

$62 \mathrm{GOI} \quad 7 \mathrm{~N} 14$

$615 W 1 \quad 7 N \quad 14$

62EDI $7 \mathrm{~N} 14$

63BAI $7 \mathrm{~N} 14$

64BII $7 \mathrm{~N} 14$

$55 \mathrm{CHI} \quad 7 \mathrm{~N} 14$

$59 M U 1 \quad 7 \mathrm{~N} 14$

6OFAl $7 \mathrm{~N} 14$

$60 \mathrm{GE} \mathrm{I} \quad 7 \mathrm{~N} 14$

$60 G E 2 \quad 7 \mathrm{~N} 14$

6OKI2 7N 14

6OSA2 $7 \mathrm{~N} 14$

$62 \mathrm{GOI} \quad 7 \mathrm{~N} 14$

$G, G$

$G, N$

$G, \times N$

$G, \times N$

$G, N P$

$G, N P$

$G, P$

$G, P$

$G, P$

$G, P$

$G, P$

$G, P$

$G, \times P$

$G, X P$

$G, X P$

$G, P A$

$G, P A$

\section{$P, G$}

$P, G$

$P, G$

$P, G$

$P, G$

$G, G$

E, E/

$E, E /$

$E, E /$

$G, N$

$G, N$

$G, N$

$G, N$

$G, N$

$G, N$

$G, N$

$G, N$
LFT 13

RLY THR 2C C 15-20

MIN-MAX TYPE MIN-MAX

SPC $10 \quad 30 \quad C \quad 31$

RLI THR $200 \quad$ C200

SPC THR 90 C 90

$S P C$

SPC THR-70

c

( 30,70

ABY 8 D 8

RLX THR $200 \quad$ C200

ABI THR 90 C 90

SPC 816 C 12,16

$S P C \quad 0 \quad 31 \quad C \quad 31$

SPC THR-31 C 15-31

$A B \times$ THR 250 C150,250

$\begin{array}{llll}A B I & 18 & 120 & C 120\end{array}$

$A B \times$ THR $22 \quad C 22$

$A B X$

$A B X$

$A B X$

$A B X$

$\begin{array}{rl}2 & 3 \\ 8 & 11 \\ 15 & 20 \\ 11 & 41 \\ 2 & 4\end{array}$

D $0-1$

D $6-9$

D $14-20$

D $10-49$

D $1-2$

$G, M U-T A B X$

$G, M U-T$ AB I

\section{$\begin{array}{lllll}7 & 21 & 0 & 20-21\end{array}$ \\ $\begin{array}{llll}0 & 170 & C 170\end{array}$}

$2 \quad D \quad 2$

$0 \quad 10 \quad 0 \quad 42$

$\begin{array}{llll}0 & 18 & 0 & 42\end{array}$

08

D 120,180

$10-16$

$\begin{array}{ll}11 & 17\end{array}$

C $10-16$

C $11-17$

$A B X$

$\begin{array}{ll}11 & 61\end{array}$

c $10-12$

RLY

$10 \quad 12$

c $10-11$

$A B X$

$\begin{array}{lll}10 & 11\end{array}$

C $10-25$

RLY 1013 C 10-13

$A B I$ c $13-61$
NAI $-D$

$A C T-I$

$\mathrm{CCH}-\mathrm{D}$

SCI -0

CCH-D

$\mathrm{CCH}-\mathrm{D}$

EMU-D

EMU-D

I ON-D

$\mathrm{CCH}-\mathrm{D}$

$\mathrm{CCH}-\mathrm{C}$

EMU-D $1-8$

$\mathrm{CCH}-\mathrm{D} 100-$

SCI -D 2-20

SCI -D 2-22

$E M U-D$

EMU-D

EMU-D

NAI $-D$

NAI -D

NAI $-C$

NAI $-D$

NAI -D

NAI $-D$

$\mathrm{CCH}-\mathrm{D}$

NAI $-D$

$T E L-D$

MAG-D 25-42 180 G-WDTH

$M A G-D$

$A C T-I$

$A C T-I$

BF 3-I

$A C T-I$

$A C T-I$

$A C T-I$

$A C T-I$

$C C H-D$
$100 \mathrm{ABI}$

4PI BREAKS

DST

90

DST

DST PROTON SPECTRUM

DST

DST * G-WIDTH

DST

DST

DST

90

$90 \mathrm{ABX}$

DST \# SPC

4PI

DST

90

90

90

DST

DST FORM FACTORS

4PI * ALSO USED BF 3

$4 \mathrm{PI}$

4PI

$4 \mathrm{PI}$

4PI E-THRESHOLD

$4 \mathrm{PI}$

$4 P I$

4PI 
$62 \mathrm{KO}$

$590 \mathrm{Cl}$

56 WR 1

$62 \mathrm{GOI}$

$62 \mathrm{KO} 2$

$55 \mathrm{CO} 1$

$62 \mathrm{KO} 2$

$62 \mathrm{GOI}$

$56 W R 1$

$58 \mathrm{CO} 2$

$62 \mathrm{GO}$

$56 L I 1$

56WR 1

$55 \mathrm{COI}$

56 WR 1

$62 \mathrm{GO} 1$

$55 C 01$

56WR 1

6 OHE 2

6ORO 1

6ORO2

$61 \mathrm{HE} 1$

$61 \mathrm{SE} 1$

63PR 1

$58 \mathrm{RH} 1$

$64 \mathrm{KO} 3$

63 F I 3
NUCLIDE REACTION RES

2 A

$7 N 14$

$7 \mathrm{~N} 14$

$7 N 14$

$7 \mathrm{~N} 14$

$7 \mathrm{~N} 14$

$7 \mathrm{~N} 14$

$7 \mathrm{~N} 14$

$7 \mathrm{~N} 14$

$7 N 14$

$7 \mathrm{~N} 14$

$7 N \quad 14$

$7 \mathrm{~N} 14$

$7 \mathrm{~N} 14$

$7 \mathrm{~N} 14$

$7 \mathrm{~N} 14$

$7 N 14$

$7 N 14$

$7 N 14$

$7 \mathrm{~N} 14$

$7 \mathrm{~N} 14$

$7 N \quad 14$

$7 N 14$

$7 \mathrm{~N} 14$

$7 N 14$

$\begin{array}{ll}7 N & 15 \\ 7 N & 15 \\ 7 N & 15\end{array}$

\section{IN,OUT}

$G, N$

$G, 2 N$

$G, N P$

$G$,NP

$G, N P$

$G, N P 3 A$

$G, N P 3 A$

$G, N A$

$G, P$

$G, P$

$G, P$

$G, P A$

$G, P A$

$G, D 3 A$

$G, A$

$G, A$

$G, 2 A$

$G, 2 A$

$P, G$

$P, G$

$P, G$

$P, G$

$P, G$

$P, G$

$G, P$

$G, P$

$G, X P$
$\begin{array}{ccc}A B X & 11 & 90 \\ \text { RLI } & T H R-100\end{array}$

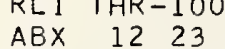

$A B I$

12170

C $19-23$

$A B X \quad 1690$

170

THR 70

c 70

C 70

RLY $20 \quad 90$

$A B I$

$A B X$

NOX

$A B I$

717

723

C 90

C 170

C 19-23

C 18-30

7170

C 170

$A B X \quad 25 \quad 50$

c 25-70

$A B X \quad 1723$

c 19-23

THR 70

C 70

$A B X \quad 1223 \quad C \quad 19-23$

$\begin{array}{llll}A B I & 12 & 170 & C 170\end{array}$

ABX THR 70

C 70

$A B X \quad 16 \quad 23$

C 19-23

$\mathrm{ABX} \quad 8$

D $0-1$

D 2

NOX

9
10

\begin{tabular}{lr}
$A B Y$ & 10 \\
\hline$L Y$ & 6
\end{tabular}

RLY 6,9

D 2-3

D $0-1$

D 2

NOX 9

D 2

ABX 1125

C 19,25

SPC $12 \quad 31$

c 19-31

C 25,31
DETECTOR

TYPE MIN-MAX

CCH-D

$A C T$ - I

$\mathrm{CCH}-\mathrm{D}$

$\mathrm{CCH}-\mathrm{D}$

$C \mathrm{CH}-\mathrm{D}$

EMU-D

$\mathrm{CCH}-\mathrm{D}$

$\mathrm{CCH}-\mathrm{D}$

$\mathrm{CCH}-\mathrm{D}$

EMU-D

$\mathrm{CCH}-\mathrm{D}$

EMU-I

CCH-D

EMU-D

CCH-D

$\mathrm{CCH}-\mathrm{D}$

EMU-D

CCH-D

NA I -D

NAI -D

NAI -D

NAI -D

MGP-D

NAI -D

$2-15$

DST

4DI REL TO G, N

$4 P I$

$4 \mathrm{PI}$

DST

4P I

$4 \mathrm{PI}$

4P I

90

4P I

4PI

$4 P I$

4PI

4P I

DST G-WDTH

DST J-PI

DST J-P I

DST J-PI

DST G-WDTH, AMPL RATIO

EMU-D $\quad 1-8 \quad$ DST

$S C I-D \quad 90 \mathrm{ABX}$

$S C I-C \quad 3-13$

$90 \mathrm{ABI}$, SEP I SOTOPES

OXYGEN $Z=8$

A ABUND.(1)

$\begin{array}{cc}16 & 99.76 \\ 17 & 3.7(-2) \\ 18 & .20\end{array}$

(1) ABUNDANC

$G, N$
15.7
4.1
8.0

\section{SEPARAT ION}

SEPARATION
$G, P$ G, T

EN

$\begin{array}{cr}\text { NERGIES } & \text { (MEV) } \\ \text { G.HE3 } & \text { G.A } \\ 22.8 & 7.2 \\ 18.8 & 6.4 \\ 25.6 & 6.2\end{array}$
15.9
15.8
25.6

18.6

EV)
G.A
7.2
6.4
6.2
$\mathrm{G}, 2 \mathrm{~N}$
28.9
19.8

G,NP

16.3

$21 \cdot 9$

$G, 2 P$
$22 \cdot 3$
$25 \cdot 3$
$29 \cdot 0$

\section{REF}

59PE 2

6OWY 1

64TE 1

59PE5

GORE 1

S9PE 5

60 IS 1

61 I 51

63 BI 2

$64 \mathrm{~B} \mathrm{I} 2$

$64 \mathrm{GO} 2$

$59 M I 2$

6OKU2

$61 B R 1$

62 B I 4

62 FU 5

64BR 1
NUCLIDE REACTION RES EXCIT

$$
Z \text { A IN,OUT }
$$

$$
80
$$

80

80

80

80

80

80

80

80

80

80

80

80

80

80

80

80
G, MU-T ABX THR 30 G, MU-T ABX 1835

$G, M U-T \quad A B X \quad 2022$

$G, G$

$G: G$

$G, G /$

$E, E /$

$E, E /$

$E, F /$

$E, E /$

$E, E /$

$G, N$

$G, N$

$G, N$

$G, N$

$G, N$

$G, N$

SOURCE

DETECTOR ANG REMARKS

MIN-MAX TYPE MIN-MAX 
REF

$64 \mathrm{TA} 4$

$57 B A 3$

$63 \mathrm{CO} 3$

$63 \mathrm{FUl}$

$64 \mathrm{~F} \mathrm{I} \mathrm{I}$

$64 Y Y_{1} 1$

$62 \mathrm{BI} 4$

55001

56001

$56 \mathrm{JO} 1$

$565 \mathrm{ST} 1$

$57 \mathrm{JO} 1$

$57 M I 3$

$61 \mathrm{FII}$

$615 \mathrm{H}^{3}$

$57 \mathrm{BR} 1$

$57 \mathrm{MII}$

61 HE2

63 F I 4

$58 \mathrm{MAl}$

$62 \mathrm{MO} 2$

$62 \mathrm{BI} 4$

$57 \mathrm{JO} 1$

$64 \mathrm{TO} 2$

$58 \mathrm{MA} 1$

$64 \mathrm{TO} 2$

$59 \mathrm{CO} 2 \quad 80 \quad 15$

61CO1 8015

$63 \mathrm{BA} 2$

$63 \mathrm{HE} 1$

585 I 3

$60 \mathrm{CA} 1$

$60 Z$ I I

$62 \mathrm{BU} 1$

$63 \mathrm{BU} 1$

$57 \mathrm{SW} 1$

62 SEl

62 BI 2

$62 \mathrm{BI} 3$

$62 E D 1$

$63 \mathrm{BA} 1$

63 I 1

$64 B 13$

$55 \mathrm{CAl}$

55PE 1

$57 \mathrm{CA} 2$

$57 \mathrm{ER} 1$

$57 S P 2$

$57 \mathrm{SV} 1$

$58 B E 1$

$59 \mathrm{~K} \mathrm{II}$

$59 M I 3$

59PE3

$595 A 2$

60GE 1

6OGE 2

6OSAl

$61 \mathrm{KE} 1$

$61 \mathrm{RO} 2$

80

80

80

80

80

80

80

80

80

80

80

80

80

80

80

80

80

80

80

80

80

80

80

80

80

80

$\begin{array}{ll}80 & 15 \\ 80 & 15\end{array}$

$80 \quad 15$

$80 \quad 16$

8016

8016

8016

8016

8016

8016

8016

8016

8016

8016

8016

8016

8016

8016

8016

8016

8016

8016

8016

$80 \quad 16$

8016

8016

8016

8016

$80 \quad 16$

8016

8016

8016
NUCLIDE REACTION RES EXCIT $Z$ A IN,OUT

$G, \times N$

$G, \times N$

$G, \times N$

$G, X N$

$G, X N$

$E, N$

$G, N P$

$G, N P$

$G, P$

$G, P$

$G, P$

$G, P$

$G, P$

$\Phi G, P$

$G, X P$

$G, X P$

$G, X P$

$G, X P$

$G, P A$

$G, P A$

$G, T$

$G, A$

$G, A$

$G, 4 A$

G, 4A

SPC THR 31

SPC THR 32

SPC THR 35

SPC

$S P C$

RLY THR-30

AEI THR 35

SPC THR 21

SPC $10 \quad 19$

NOX THR 30

SPC 035

SPC 1430

$A B \times$ THR 22

$A B \times$ THR 22

$P, G$

$P, G$

$P, G$

NOX 8

$P, G$

LFT

8
8

G, MUIT ABX 2021

$G, M U-T$ ABX 1226

G,MU-T ABX 1927

$G, G$

$G, G$

$E, E /$

$E, E /$

$E, E /$

$E, E /$

$E, E /$

$G, N$

$G, N$

$G, N$

$G, N$

$G, N$

$G, N$

$G, N$

$G, N$

$G, N$

$G, N$

$G, N$

$G, N$

$G, N$

$G, N$

$G, N$

$G, N$

$\begin{array}{lll}A B X & 17 & 18\end{array}$
$G, N \quad$ SPC 1626

ABX $\quad 16 \quad 75$

$A B Y \quad 60 \quad 150$

THR-252

RLX THR-340

$A B X \quad 1226$

ABX. THR $250 \quad$ C 150,250

$23120 \quad C 120$

$A B Y 60150$ C 60-150

SPC 719

C 19-

C 22

$A B X$ THR $250 \quad C 150,250$

c 22

$A B \times \quad 1925$

$A B \times \quad 1925$

D $14-20$

D $14-20$

D 1

D $0-1$ NAI - D

G,MU-T RLX 1625

C 15-25 ACT-I

$G, M U-T \quad A B X \quad 1226$

$E, E /$
LFT 7,7

NOX 7

FMF 19

FMF $\quad 44,49$

NOX 016

ABX 19

NOX 2226

D 20-21

C 30

C250

C250

D 7,7

C 16

D 140-215 MAG-D

0150

D 41

D 41

MGP-D $12-26$

MGP-D $19-30$

$M G P-D \quad 12-26$

$N A I-D$

NAI -D

$M A G-D$

$M A G-D$

$M A G-D$
$M A G-D$

D 100-218 MAG-D

$\begin{array}{cccccc}A B X & 17 & & D & 0 & A C T-I \\ A B I & 15 & 23 & C & 16-23 & A C T-I\end{array}$

$A B I \quad 15 \quad 23$

c $15-30$

C 16-32

$A C T-I$

$A B X \quad 16 \quad 32$

RLX $16 \quad 25$

ABX $20 \quad 25$

C 14-25

$A C T-I$

RLY 1517

ABI 1518

C 16-30

NAI $-D$

C 15-17 ACT-I

C 15-19 ACT-I

NOX $16 \quad 31$

NOX $16 \quad 17$

C 31

EMU-D

$A C T-I$

$A C T-I$

ABX $15 \quad 17$

C 15-17 ACT-I

NOX 1517

C 15-17

$A C T-I$

NOX 1516

C 15-16

$A C T-I$

D $17-18$

$A C T-I$

$A C T-1$

ANG REMARKS

0

90

$4 \mathrm{PI}$

90

70

90 BRANCHING RATIOS

4P I MULTIPOLAR ITY

DST *

DST *

DST * 


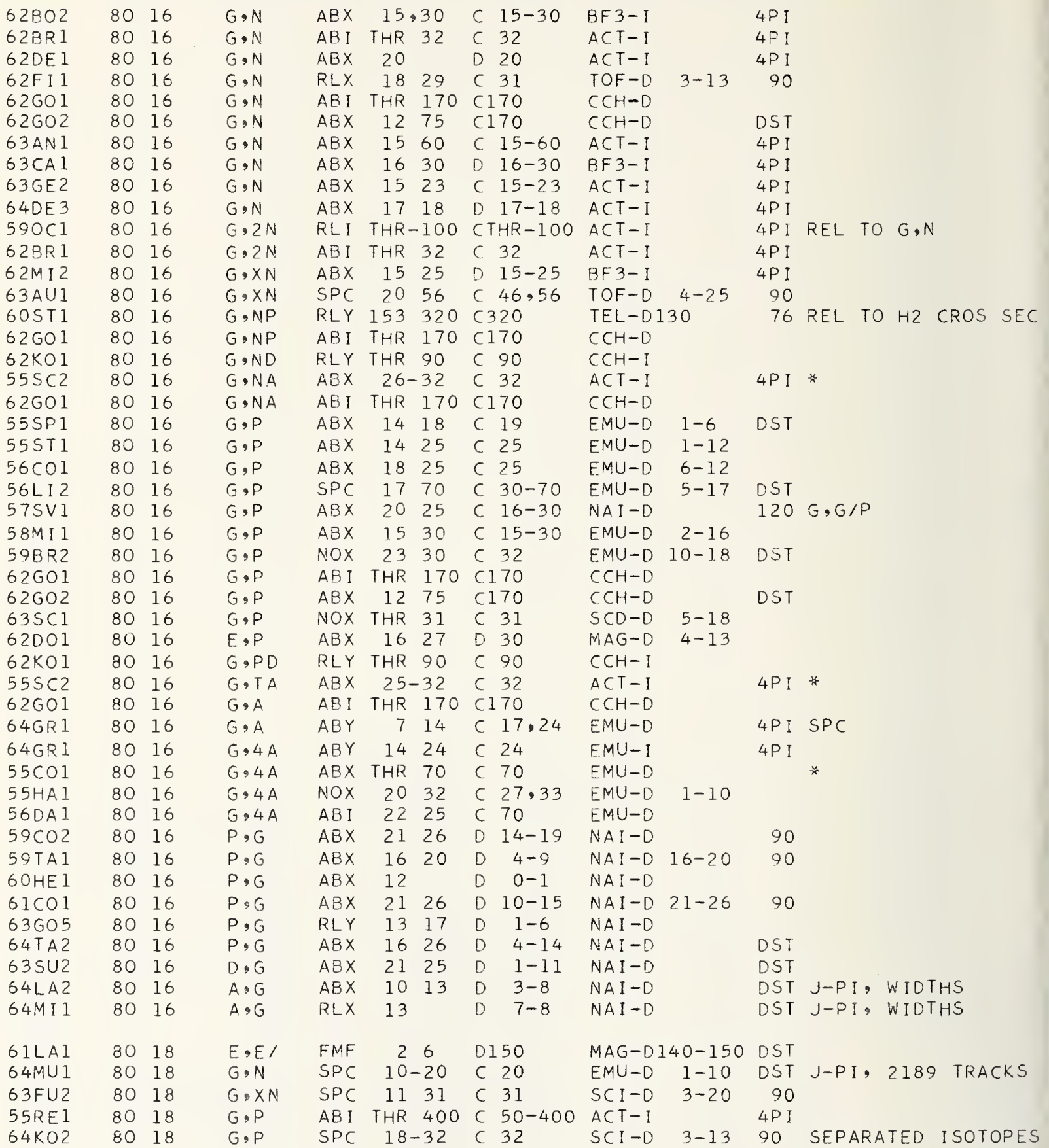



A ABUND.
SEPARATION ENERGIES (MEV)

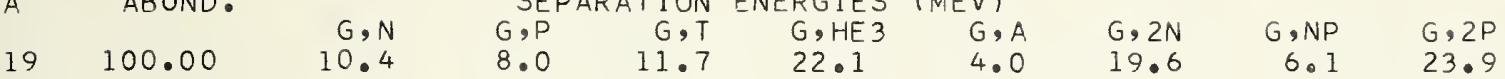

RE

NUCLIDE REACTION RES

EXCIT

SOURCE DETECTOR ANG REMARKS $Z$ A IN,OUT MIN-MAX TYPE MIN-MAX

\begin{tabular}{|c|c|c|c|c|c|c|c|c|c|c|c|c|}
\hline & & & 190 & & & & & IAA & TYPE 1 & $M A$ & & \\
\hline 63SE 1 & $9 F$ & 17 & $P, G$ & FLY & 4 & & D & 3 & $N A I-D$ & & DST & J-PI, WIDTH \\
\hline $64 T E 1$ & $9 \mathrm{~F}$ & 19 & $G, M U-T$ & $A B X$ & 20 & 21 & $D$ & $20-21$ & $N A I-D$ & & 96 & \\
\hline 60803 & $9 \mathrm{~F}$ & 19 & $G, G$ & LFT & 1 & & $C$ & $0-2$ & NAI - D & & 100 & $J-P I$ \\
\hline 6ORE 1 & $9 \mathrm{~F}$ & 19 & $G, G$ & $A B X$ & 7 & & $D$ & 7 & NAI -D & & 90 & \\
\hline $62 B 06$ & $9 \mathrm{~F}$ & 19 & $G, G$ & LFT & 0 & 4 & C & $0-4$ & NAI -D & & DST & \\
\hline $64 \mathrm{BO} 1$ & $9 \mathrm{~F}$ & 19 & $G, G$ & LFT & 1 & 3 & C & $1-3$ & NAI - D & & 100 & $A B I$ \\
\hline $63 B A 1$ & $9 \mathrm{~F}$ & 19 & $E, E /$ & SPC & 0 & 16 & 0 & 42 & $M A G-D$ & & 180 & \\
\hline $58 \mathrm{BE} 1$ & $9 \mathrm{~F}$ & 19 & $G, N$ & NOX & 10 & 11 & C & $10-11$ & $A C T-I$ & & $4 \mathrm{PI}$ & THRESHOLD \\
\hline $60 \mathrm{GE} 2$ & $9 \mathrm{~F}$ & 19 & $G, N$ & NOX & 10 & 11 & $c$ & $10-11$ & $A C T-I$ & & $4 \mathrm{PI}$ & THRESHOLD \\
\hline $60 \mathrm{KI} 4$ & $9 \mathrm{~F}$ & 19 & $G, N$ & $A B X$ & 10 & 23 & C & $10-23$ & $A C T-I$ & & 4P I & \\
\hline $605 A 2$ & $9 \mathrm{~F}$ & 19 & $G, N$ & $R L Y$ & 10 & 12 & C & $10-12$ & $A C T-I$ & & 4PI & \\
\hline 6OWA2 & $9 \mathrm{~F}$ & 19 & $G, N$ & $A B X$ & 10 & 240 & C. & $120-240$ & $A C T-I$ & & 4P I & \\
\hline $620 E 1$ & $9 \mathrm{~F}$ & 19 & $G, N$ & $A B X$ & 20 & 21 & D & $20-21$ & $A C T-I$ & & 4P I & \\
\hline $590 C 1$ & $9 \mathrm{~F}$ & 19 & $G, 2 N$ & RL I & THR - & -100 & & THR -100 & $A C T-I$ & & 4PI & REL TO $G, N$ \\
\hline $60 \mathrm{FO} 2$ & $9 \mathrm{~F}$ & 19 & $G, P$ & $R L X$ & 10 & 18 & C & 16,19 & $E M U-D$ & & DST & \\
\hline S2BR3 & $9 \mathrm{~F}$ & 19 & $G, P$ & $S P C$ & 18 & & D & 18 & EMU-D & $2-10$ & DST & \\
\hline 63MUI & $9 \mathrm{~F}$ & 19 & $G, P$ & $S P C$ & 11 & 24 & $c$ & 24 & $S C D-D$ & $3-11$ & 120 & \\
\hline 62001 & $9 \mathrm{~F}$ & 19 & $E, P$ & SPC & 10 & 19 & $D$ & $18-30$ & $M A G-D$ & $2-10$ & DST & \\
\hline $55 R E 1$ & $9 \mathrm{~F}$ & 19 & $G, 2 P$ & $A B I$ & THR & 400 & C & $80-400$ & $A C T-I$ & & 4P I & \\
\hline $55 \mathrm{LA} 1$ & $9 \mathrm{~F}$ & 19 & $G, \times P$ & $S P C$ & & -17 & & $10-17$ & & & DST & * AB I \\
\hline
\end{tabular}

NEON $Z=10$

\begin{tabular}{|c|c|c|c|c|c|c|c|c|c|}
\hline A & ABUND. & & SE & ATION & ENERG I ES & (MEV) & & & \\
\hline & & $G, N$ & $G, P$ & $G, T$ & G, HE 3 & $G, A$ & $G, 2 N$ & G,NP & G,2P \\
\hline $\begin{array}{l}20 \\
21\end{array}$ & $\begin{array}{r}90.92 \\
0.26\end{array}$ & $\begin{array}{r}16.9 \\
6.8\end{array}$ & $\begin{array}{l}12.8 \\
13.0\end{array}$ & $\begin{array}{l}23.9 \\
21.6\end{array}$ & $\begin{array}{l}21.2 \\
19.9\end{array}$ & $\begin{array}{l}4.7 \\
7.3\end{array}$ & $\begin{array}{l}28.5 \\
23.6\end{array}$ & $\begin{array}{l}23.3 \\
19.6\end{array}$ & \\
\hline 22 & 8.82 & 10.4 & 15.3 & 21.5 & 26.3 & 9.7 & 17.1 & 23.4 & 26. \\
\hline
\end{tabular}

REF NUCLIDE REACTION RES EXCIT SOURCE DETECTOR ANG REMARKS

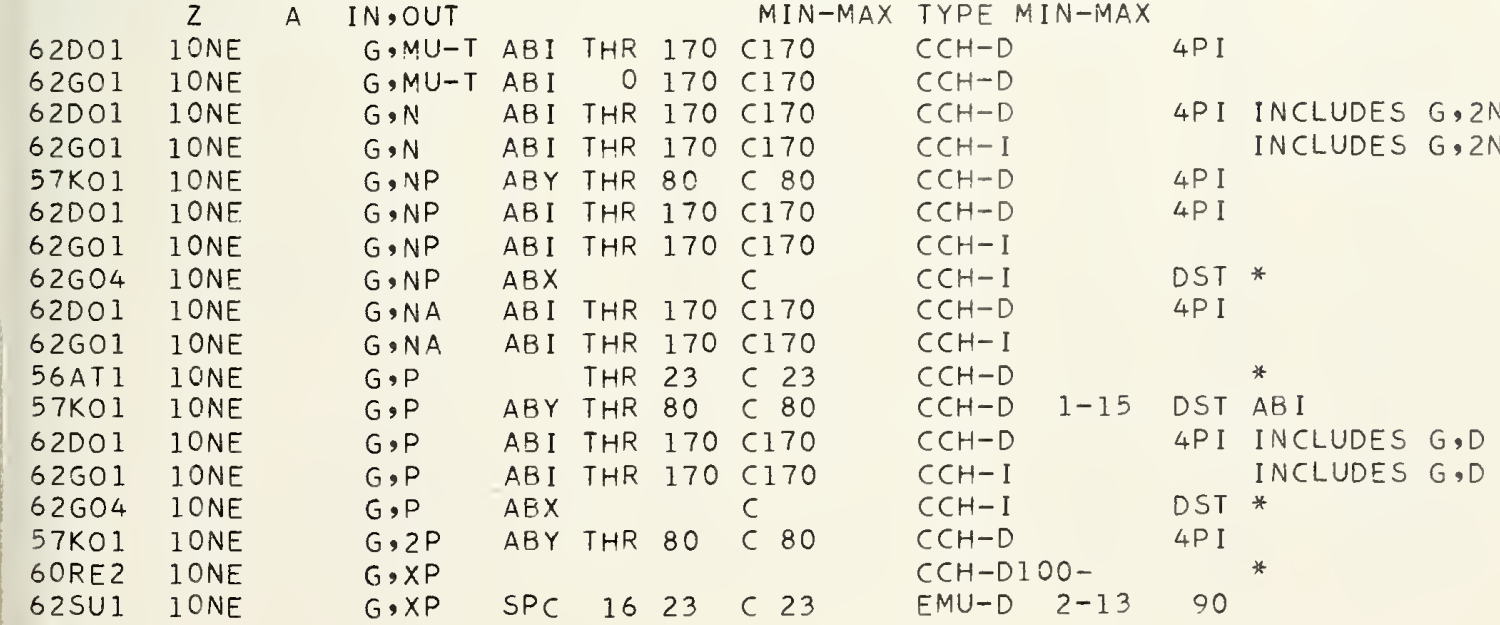




\begin{tabular}{|c|c|c|c|c|c|c|c|c|c|c|c|c|}
\hline REF & NUCL IDE & REACTION & RES & EX & CI T & & SOURCE & DET & IECTOR & ANG & REMARKS & \\
\hline & Z A & IN,OUT & & & & & MIN-MAX & TYPE & MIN-MAX & & & \\
\hline F I 4 & IONE & $G, X P$ & SPC & 15 & 29 & $C$ & 31 & $S C I-D$ & $2-15$ & 90 & & \\
\hline ATI & IONE & $G, P A$ & & THR & 23 & $c$ & 23 & $\mathrm{CCH}-\mathrm{D}$ & & & * & \\
\hline $\mathrm{GO4}$ & 1ONE & $G, P A$ & $A B X$ & & & $c$ & & $\mathrm{CCH}-\mathrm{I}$ & & DST & * & \\
\hline AT 1 & 1ONE & $G, A$ & & THR & 23 & $c$ & 23 & $\mathrm{CCH}-\mathrm{D}$ & & & * & \\
\hline $\mathrm{GO1}$ & IONE & $G, A$ & $A B I$ & THR & 170 & $\mathrm{Cl}$ & 170 & $\mathrm{CCH}-\mathrm{I}$ & & & & \\
\hline $\mathrm{KO}$ & IONE & $\mathrm{G}, 5 \mathrm{~A}$ & $A B Y$ & THR & 80 & $C$ & 80 & $\mathrm{CCH}-\mathrm{D}$ & & 4PI & & \\
\hline RE I & IONE & $G, N 17$ & AB I & THR & 400 & $c$ & $90-400$ & $A C T-I$ & & 4PI & & \\
\hline$B A 1$ & 1 ONE 20 & $E, E /$ & SPC & 0 & 16 & $D$ & 42 & $M A G-D$ & & 180 & J-PI, WIDTHS, & $A B X$ \\
\hline HA 1 & $10 N E 20$ & $G, N$ & $A B X$ & 15 & 18 & $D$ & 15,18 & I ON-D & & $4 P I$ & & \\
\hline HAl & 1ONE 20 & $G, 2 A$ & $A B X$ & 15 & 18 & $D$ & 15,18 & I ON-D & & 4PI & & \\
\hline 3R 1 & $10 N E 20$ & $P, G$ & RLY & 17 & 23 & $D$ & $4-11$ & NAI I D & & & & \\
\hline$A L 3$ & 1ONE2O & $P, G$ & NOX & 17 & 22 & $D$ & $4-9$ & NA I -D & & DST & & \\
\hline TA 1 & 1ONE 20 & $P, G$ & $A B X$ & 17 & 24 & $D$ & $4-11$ & NAI - D & & 90 & & \\
\hline 02 & 1ONE 22 & $G, N P$ & SPC & THR & 90 & $C$ & 90 & $\mathrm{CCH}-\mathrm{D}$ & $2-20$ & DST & SEPARATED ISO & TOP \\
\hline 02 & 1ONE22 & $G, P$ & SPC & THR & 90 & $c$ & 90 & $\mathrm{CCH}-\mathrm{D}$ & $2-24$ & DST & SEPARATED ISO & \\
\hline $1 A_{1}$ & $10 N E 22$ & $G, A$ & $A B X$ & 15 & 18 & $D$ & 15,18 & I ON-D & & $4 \mathrm{PI}$ & & \\
\hline
\end{tabular}

$$
\text { SODIUM } Z=11
$$

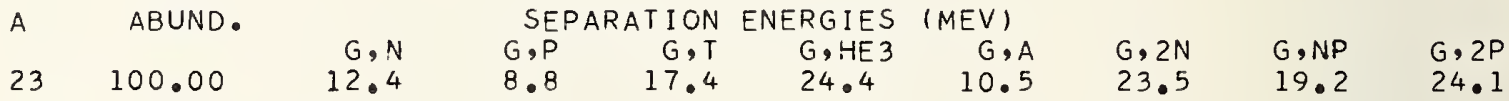

\begin{tabular}{|c|c|c|c|c|c|c|c|c|c|c|c|c|}
\hline $\begin{array}{l}\text { REF } \\
\text { G1BE2 }\end{array}$ & $\underset{Z}{\text { NUCL IDE }}$ & $\begin{array}{c}\text { REACT ION } \\
\text { IN, OUT }\end{array}$ & RES & \multicolumn{2}{|c|}{ EXCI T } & \multicolumn{2}{|c|}{ SOURCE } & \multicolumn{2}{|c|}{ DETECTOR } & ANG & \multicolumn{2}{|l|}{ REMARKS } \\
\hline $56 \mathrm{FUI}$ & 11 NA 23 & $G, G$ & $A B X$ & 4 & 40 & $C$ & $4-40$ & NAI - D & & 120 & ERRATUM PR & 106,93 \\
\hline 59RA 1 & $11 \mathrm{NA} 23$ & $G, G$ & LFT & 1 & & D & 1 & NAI -D & & DST & & \\
\hline $62 M O 1$ & $11 N A 23$ & $G, G$ & LFT & 1 & & $C$ & 1 & NAI - D & & 130 & & \\
\hline $64 \mathrm{ME} 1$ & 11 NA2 23 & $G, G$ & LFT & 4 & & $D$ & 4 & NAI -D & & DST & J-PI, WIDTH & \\
\hline $64 \mathrm{SW} 1$ & 11 NA 23 & $G, G$ & NOX & 7 & & $D$ & 7,7 & $N A I-D$ & & DST & J-PI, WIDTH & \\
\hline $63 \mathrm{BA} 1$ & $11 N A 23$ & $E, E /$ & SPC & 0 & 20 & $D$ & 42 & $M A G-D$ & & 180 & $A B X$ & \\
\hline $58 \mathrm{CH} 2$ & 11 NA 23 & $G, N$ & RLY & THR & & $C T$ & THR & $B F 3-I$ & & 4P I & THRESHOLD & \\
\hline $58 K E 1$ & 11 NA2 3 & $G, P$ & SPC & 18 & & $D$ & 18 & $N A I-D$ & $3-8$ & 4P I & $A B X$ & \\
\hline $580 P 1$ & 11 NA23 & $G, P$ & SPC & 18 & & $D$ & 18 & NAI $-D$ & $3-8$ & 4P I & $A B X$ & \\
\hline 62001 & 11 NA 23 & $G, P$ & SPC & 11 & 18 & $c$ & 18 & $E M U-D$ & $2-10$ & DST & $A B Y$ & \\
\hline 55RE1 & $11 N A 23$ & $G, N 17$ & $A B I$ & THR & 400 & $C$ & $90-400$ & $A C T-I$ & & $4 \mathrm{PI}$ & & \\
\hline $62 B R 2$ & $11 N A 23$ & $P, G$ & NOX & 9 & 10 & $D$ & $0-1$ & NAI I D & & DST & $J-P I$ & \\
\hline
\end{tabular}




\begin{tabular}{|c|c|c|c|c|c|c|c|c|c|}
\hline A & ABUND. & & SE & ATION & ENERGIES & (MEV) & & & \\
\hline & & $G, N$ & $G, P$ & $G, T$ & $G, H E 3$ & $G, A$ & $G, 2 \mathrm{~N}$ & $G, N P$ & $G, 2 P$ \\
\hline 24 & 78.70 & 16.5 & 11.7 & 26.7 & 23.1 & 9.3 & 29.9 & 24.1 & 0.5 \\
\hline 25 & 10.1 & 7. & 12.1 & 23.0 & 20.1 & 9.9 & 23.9 & 19.1 & 2.5 \\
\hline 26 & 11.17 & 11.1 & 14.1 & 21.6 & 26.0 & 10.6 & 18.4 & 23.2 & 4. \\
\hline
\end{tabular}

REF NUCLIDE REACTION RES EXCIT SOURCE DETECTOR ANG REMARKS

\begin{tabular}{|c|c|c|c|c|c|c|c|c|c|c|c|}
\hline & A & IN, OUT & & & & & IIN-MAX & TYPE & IN-MAX & & \\
\hline $56 \mathrm{FU1}$ & $12 M G$ & $G, G$ & $A B X$ & 4 & 40 & c & $4-40$ & $N A I-D$ & & 120 & ERRATUM PR 106,99 \\
\hline $588 \cup 1$ & $12 M G$ & $G, G$ & & 1 & & D & 1 & & & 4PI & * WIDTH \\
\hline 59LAl & $12 M G$ & $G, G$ & & & & c & 13 & & & & * \\
\hline 59VAl & $12 M G$ & $G, G$ & & 1 & 4 & & & & & & * \\
\hline $60 B \cup 3$ & $12 M G$ & $G, G$ & $A B X$ & 11 & & c & 11 & & & DST & * WIDTH MULTIPOL. \\
\hline 6ISUl & $12 M G$ & $G, G$ & RLY & 7 & 14 & c & $7-14$ & NAI I D & & 120 & \\
\hline $62 \mathrm{SE} I$ & $12 M G$ & $G, G$ & NOX & 0 & 16 & $c$ & 16 & $N A I-D$ & & 90 & \\
\hline $63 S U 1$ & $12 M G$ & $G, G$ & $A B X$ & 4 & 14 & c & $4-14$ & NAI I D & & 120 & \\
\hline $64 \mathrm{GO} 3$ & $12 M G$ & $E, E /$ & $A B X$ & 11 & & D & $40-70$ & $M A G-D$ & & 180 & FMF \\
\hline 55 YE 1 & $12 M G$ & $G, N$ & $A B X$ & 11. & -17 & & $11-17$ & & & & * \\
\hline $58 S P 2$ & $12 M G$ & $G, N$ & $A B X$ & 8 & 17 & $c$ & $8-17$ & BF 3-I & & $4 \mathrm{PI}$ & \\
\hline 56YEI & $12 M G$ & $G, \times N$ & $A B Y$ & 7 & 23 & c & $7-23$ & BF 3-I & & $4 P I$ & \\
\hline $63 \mathrm{CO}_{3}$ & $12 M G$ & $G, \times N$ & $A B X$ & 10 & 80 & c & $10-80$ & SF 3-I & & 4PI & \\
\hline $62 \mathrm{SHII}$ & $12 M G$ & $G, P$ & $A B X$ & 15 & 24 & CT & $H R-24$ & $S C I-D$ & $1-$ & DST & \\
\hline $64 \mathrm{FO} 1$ & $12 M G$ & $G, P$ & SPC & THR & 40 & $c$ & $20-40$ & $E M U-D$ & $1-18$ & DST & $R L x$ \\
\hline 64 I S I & $12 M G$ & $G, X P$ & $A B X$ & THR- & -32 & c & $15-32$ & $S C I-D$ & $0-3$ & & \\
\hline 55RE 1 & $12 M G$ & $\mathrm{G}, N 17$ & $A B I$ & THR & 400 & c & $80-400$ & $A C T-I$ & & $4 \mathrm{PI}$ & \\
\hline $608 \cup 2$ & $12 M G 24$ & $G, G$ & $A B X$ & 11 & & c & 11 & & & DST & * WIDTH, MULTIPOL \\
\hline 6OME & $12 M G 24$ & $G, G$ & NOX & 1 & & $D$ & 1 & $N A I-D$ & & DST & J-PI, WIDTH \\
\hline 60TO1 & $12 M G 24$ & $G, G$ & NOX & 9 & 11 & $c$ & 13 & NAI -D & & 118 & THRESHOLD \\
\hline 62806 & $12 M G 24$ & $G, G$ & LFT & 0 & 4 & $c$ & $0-4$ & NAI I D & & DST & \\
\hline 64801 & $12 M G 24$ & $G, G$ & LFT & 1 & 3 & $c$ & $1-3$ & NAI I D & & 100 & AB I \\
\hline $63 \mathrm{BAl}$ & $12 M G 24$ & $E, E /$ & $S P C$ & 0 & 16 & $D$ & 42 & $M A G-D$ & & 180 & J-PI, WIDTHS \\
\hline $60 \mathrm{KII}$ & $12 M G 24$ & $G, N$ & $A B X$ & 16 & 25 & $c$ & $16-25$ & $A C T-I$ & & $4 P I$ & \\
\hline $55 \mathrm{NAl}$ & $12 M G 24$ & $G, \times N$ & $A B X$ & 16 & 24 & $c$ & $9-24$ & BF 3-I & & $4 P I$ & \\
\hline 63 YAl & $12 M G 24$ & $G, P$ & $S P C$ & 15 & 22 & $c$ & 22 & $E M U-D$ & $2-10$ & DST & $R L Y$ \\
\hline 6OWA2 & $12 M G 24$ & $G, 2 N 3 P$ & $A B X$ & THR & 240 & $c 2$ & 240 & $A C T-I$ & & 4PI & \\
\hline $63 \mathrm{SH}^{3}$ & $12 M G 24$ & $\mathrm{G}, \mathrm{C} 12^{*}$ & $R L Y$ & THR & 70 & $c$ & 70 & $E M U-D$ & & $4 P I$ & 15 6-ALPHA EVENTS \\
\hline $61 \mathrm{GOl}$ & $12 M G 24$ & $P, G$ & $R L Y$ & 15 & 23 & $D$ & $3-11$ & $N A I-D$ & & 90 & \\
\hline $62 \mathrm{GL} 1$ & $12 M G 24$ & $P, G$ & NOX & 12 & & $D$ & $0-1$ & NAI I D & & 55 & \\
\hline $62 P R 1$ & $12 M G 24$ & $P, G$ & NOX & 12 & 13 & $D$ & $0-1$ & $N A I-D$ & & 55 & WIDTHS \\
\hline $63 \mathrm{GO} 3$ & $12 M G 24$ & $P, G$ & $R L Y$ & 15 & 23 & $D$ & $4-12$ & $N A I-D$ & & 90 & \\
\hline 6OME 1 & $12 M G 25$ & $G, G$ & NOX & 2 & & $D$ & 2 & NAI -D & & DST & J-PI, WIDTH \\
\hline & $12 M G 25$ & $G, G$ & LFT & 2 & & $D$ & 2 & NAI -D & & DST & $J-P I$ \\
\hline 62806 & $12 M G 25$ & $G, G$ & LFT & 0 & 4 & $c$ & $0-4$ & $N A I-D$ & & DST & \\
\hline $64 \mathrm{BO}$ & $12 M G 25$ & $G, G$ & LFT & 1 & 3 & $c$ & $1-3$ & $N A I-D$ & & 100 & AB I \\
\hline $55 \mathrm{NA} 1$ & $12 M G 25$ & $G, \times N$ & $A B X$ & 7 & 24 & $c$ & $9-24$ & BF 3-I & & $4 \mathrm{PI}$ & \\
\hline 6 IRA 1 & $12 M G 26$ & $G, G$ & LFT & 2 & & D & 2 & NAI I D & & DST & \\
\hline 64801 & $12 M G 26$ & $G, G$ & LFT & 1 & 3 & $c$ & $1-3$ & NAI I D & & 100 & $A B I$ \\
\hline
\end{tabular}




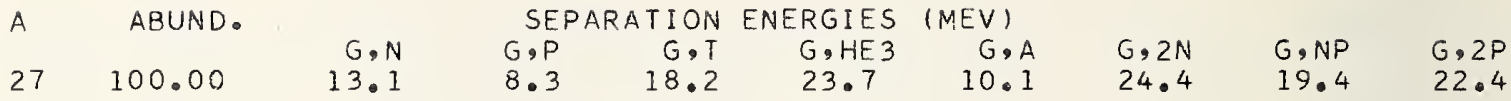

\begin{tabular}{|c|c|c|c|c|c|c|c|c|c|c|c|}
\hline REF & $\begin{array}{c}\text { NUCL IDE } \\
Z \quad A\end{array}$ & $\begin{array}{l}\text { REACTION } \\
\text { IN, OUT }\end{array}$ & RES & $E \times C$ & EIT & & $\begin{array}{l}\text { SOURCE } \\
\text { MIN-MAXX }\end{array}$ & $\begin{array}{c}\text { DET } \\
\text { TYPE }\end{array}$ & $\begin{array}{l}\text { ECTOR } \\
\text { MIN-MAX }\end{array}$ & ANG & REMARKS \\
\hline $\begin{array}{l}63 G A 1 \\
63 M C 2\end{array}$ & $\begin{array}{l}13 \mathrm{AL} 25 \\
13 \mathrm{AL} 25\end{array}$ & $\begin{array}{l}P, G \\
P, G\end{array}$ & $\begin{array}{l}\text { LFT } \\
\text { NOX }\end{array}$ & $\begin{array}{l}1 \\
2\end{array}$ & & $\begin{array}{l}0 \\
0\end{array}$ & $\begin{array}{l}1 \\
1\end{array}$ & $\begin{array}{l}S C I-D \\
\text { NAI -D }\end{array}$ & & DST & $J-P I, E 2 / M I$ RATIO \\
\hline $62 N E 2$ & 13 AL 26 & $P, G$ & NOX & 7 & 8 & D & $1-2$ & NAI $-D$ & & 0 & \\
\hline $63 G A I$ & 1. $3 A L 26$ & $P, G$ & LFT & 1 & & 0 & 1 & $S C D-D$ & & & \\
\hline $63 \mathrm{HOI}$ & $13 A L 26$ & $P, G$ & NOX & 0 & 2 & 0 & $-I$ & $N A I-D$ & & DST & J-PI, I SOSPIN \\
\hline 582 I 1 & $13 \mathrm{AL} 27$ & $G, M U-T$ & $A B X$ & 17 & 25 & $c$ & $17-30$ & $M G P-D$ & & & \\
\hline 59001 & 13AL 27 & $G, M U-T$ & $A B X$ & 13 & 30 & $c$ & 29.30 & $M G C-D$ & & & \\
\hline $59 \mathrm{KOI}$ & $13 A L 27$ & $G, M U-T$ & $A B X$ & 13 & 30 & $c$ & 30 & NAI $-D$ & & & \\
\hline 59MI 1 & $13 A L 27$ & $G, M U-T$ & $A B X$ & 13 & 30 & $c$ & 30 & $M G C-D$ & & & \\
\hline $60 C A 1$ & $13 \mathrm{AL} 27$ & $G, M U-T$ & $A B X$ & 13 & 21 & 0 & $20-21$ & NAI $-D$ & & & \\
\hline 60TA2 & $13 A L 27$ & $G, M U-T$ & $A B X$ & 7 & 28 & $c$ & 28 & $M A G-D$ & & & \\
\hline $60 W Y 1$ & $13 A L 27$ & $G, M U-T$ & $A B X$ & 13 & 55 & $c$ & $35-90$ & NAI I D & & & * \\
\hline 60211 & $13 A L 27$ & $G, M U-T$ & $A B X$ & 13 & 30 & $c$ & $10-30$ & MGP $-D$ & & & \\
\hline 64TEI & $13 A L 27$ & $G, M U-T$ & $A B X$ & 20 & 21 & 0 & $20-21$ & NAI $-D$ & & 96 & \\
\hline $56 F \cup 1$ & $13 \mathrm{AL} 27$ & $G, G$ & $A B X$ & 4 & 40 & $c$ & $4-40$ & NAI $-D$ & & 120 & ERRATUM PR 106,993 \\
\hline $58 A L 1$ & $13 \mathrm{AL} 27$ & $G: G$ & $A B X$ & 2 & & & & & & 124 & \\
\hline 6OME 1 & $13 \mathrm{AL} 27$ & $G, G$ & NOX & 1, & 2 & 0 & 1,2 & $N A I-D$ & & DST & \\
\hline 6ORE 1 & $13 \mathrm{AL} 27$ & $G, G$ & $A B X$ & 7 & & D & 7 & $N A I-D$ & & 90 & \\
\hline 6OVAI & $13 \mathrm{AL} 27$ & $G, G$ & LFT & 1 & & $c$ & 3 & & & & * \\
\hline $610 E 4$ & 13 AL 27 & $G, G$ & $A B X$ & 14 & 32 & $c$ & 32 & NAI I D & & DST & \\
\hline 61T01 & $13 \mathrm{AL} 27$ & $G, G$ & $A B X$ & 3 & 15 & $c$ & $4-15$ & $N A I-D$ & & 120 & \\
\hline $62 B 06$ & 13AL 27 & $G, G$ & LFT & 3 & & $c$ & $0-4$ & $N A I-D$ & & DST & \\
\hline 63VA3 & $13 A L 27$ & $G, G$ & NOX & 2 & & $c$ & 3 & NAI $-D$ & & DST & WIDTH \\
\hline 63VA4 & $13 A L 27$ & $G, G$ & NOX & 2 & 3 & $c$ & 4 & $N A I-D$ & & 120 & WIDTH \\
\hline $64 B 01$ & $13 A L 27$ & $G: G$ & LFT & 1 & 3 & $c$ & $1-3$ & NAI $-D$ & & 100 & $A B I$ \\
\hline 63BAI & $13 \mathrm{AL} 27$ & $E, E /$ & SPC & 0 & 18 & 0 & 42 & $M A G-D$ & $24-42$ & 180 & $A B X$ \\
\hline 63604 & $13 A L 27$ & $E, E /$ & $A B X$ & & & D & 41 & $M A G-D$ & & 180 & \\
\hline $64 \mathrm{AMI}$ & 13 AL 27 & $E, E / P$ & RLX & THR & 150 & & 550 & $M A G-D$ & & 51 & \\
\hline 55101 & $13 A L 27$ & $G, N$ & RLY & 15 & 65 & $c$ & 65 & $S C I-D$ & $2-$ & DST & \\
\hline $55 \mathrm{JOI}$ & $13 A L 27$ & $G, N$ & RLY & $23-$ & -65 & $c$ & 65 & $S C I-D$ & $10-$ & DST & \\
\hline $57 \mathrm{FEI}$ & $13 A L 27$ & $G, N$ & $R L Y$ & 22 & 31 & c & 31 & THR-I & $5-$ & DST & \\
\hline $58 \mathrm{ASI}$ & $13 A L 27$ & $G, N$ & NOX & 13 & 17 & $c$ & 17 & $S C I-I$ & & DST & \\
\hline $585 \mathrm{~A} 5$ & $13 \mathrm{AL} 27$ & $G, N$ & RLX & $12-$ & -14 & & $12-14$ & & & & * BREAKS, THRESH. \\
\hline $58 \mathrm{CH} 2$ & $13 A L 27$ & $G, N$ & $R L Y$ & THR & & & THR & $B F 3-I$ & & $4 \mathrm{PI}$ & THRESHOLD \\
\hline $58 \mathrm{FEl}$ & I3AL 27 & $G, N$ & $A B X$ & 13 & 30 & $c$ & $13-30$ & $A C T-I$ & & $4 \mathrm{PI}$ & \\
\hline $59 \mathrm{KUI}$ & $13 A L 27$ & $G, N$ & NOX & 23 & 88 & $c$ & 88 & SCI-I & $10-$ & DST & QUASI-DEUTERON \\
\hline $60 \mathrm{CH}^{2}$ & 13AL 27 & $G, N$ & $A B X$ & 13 & 21 & c & $12-21$ & $B F 3-I$ & & $4 \mathrm{PI}$ & \\
\hline 6OGE3 & $13 \mathrm{AL} 27$ & $G, N$ & NOX & THR & & & THR & $B F 3-I$ & & $4 \mathrm{PI}$ & THRESHOLD \\
\hline $60 \mathrm{KU2}$ & $13 \mathrm{AL} 27$ & $G, N$ & RLY & 23 & 90 & $c$ & 90 & $T E L-I$ & $10-$ & DST & \\
\hline 6IBAI & $13 A L 27$ & $G, N$ & $A B X$ & 13 & 19 & $c$ & $12-19$ & $B F 3-I$ & & 4PI & \\
\hline 6IPRI & $13 \mathrm{AL} 27$ & $G, N$ & RLX & 13 & 85 & c & $25-85$ & THR-D & $11-$ & & \\
\hline $62 \mathrm{BO} 2$ & $13 A L 27$ & $G, N$ & $A B X$ & 13 & 30 & $c$ & $14-30$ & BF 3-I & & $4 \mathrm{PI}$ & \\
\hline $62 M U 1$ & $13 A L 27$ & $G, N$ & $A B X$ & 13 & 24 & $c$ & $13-24$ & $B F 3-I$ & & $4 \mathrm{PI}$ & \\
\hline $63 M I 4$ & $13 A L 27$ & $G, N$ & $S P C$ & 15 & 30 & c & 24,30 & $E M U-D$ & $2-15$ & 90 & \\
\hline $55 B A 5$ & $13 A L 27$ & $G, \times N$ & $A B X$ & THR- & -250 & & 250 & & $30-$ & DST & * \\
\hline $550 I 1$ & $13 A L 27$ & $G, \times N$ & NOX & 13 & 70 & c & 70 & $S C I-I$ & & DST & \\
\hline $56 \mathrm{HEI}$ & $13 A L 27$ & $G, \times N$ & RLY & $13-$ & -31 & $c$ & 31 & & & & * REL TO TRITONS \\
\hline 56WAI & $13 A L 27$ & $G, \times N$ & RLY & $13-$ & -31 & $c$ & 31 & $A C T-I$ & & 4PI & * REL TO TRITONS \\
\hline 5EFE 1 & 13AL 27 & $G, \times N$ & $A B X$ & 13 & 30 & c & $13-30$ & THR-I & $5-$ & & FAST NEUTRONS \\
\hline 59003 & $13 A L 27$ & $G, \times N$ & SPC & 15 & 30 & $c$ & 24,30 & $E M U-D$ & $2-15$ & 90 & \\
\hline & $13 A L 27$ & $G, \times N$ & $A B X$ & 12 & 80 & C & 80 & $B F 3-I$ & & $4 P I$ & \\
\hline 6OSTI & $13 A L 27$ & $G, N P$ & RLX & 149 & 320 & & 320 & TEL-D & 130 & 76 & REL TO H2 CROS SEC \\
\hline
\end{tabular}


REF NUCLIDE REACTION RES EXCIT $Z$ A IN,OUT $60 G 02 \quad 13 A L 27$

6OWA2 13AL27

55DA1 I3AL 27

$55 \mathrm{JO} 13 \mathrm{AL} 27$

$57 R O 1$ I3AL 27

$59 B A 2$ 13AL27

$60 \mathrm{CH} 2 \quad 13 \mathrm{AL} 27$

6IMAI I3AL 27

62BR3 13AL 27

62SE2 I3AL 27

$62 \mathrm{SH} 8 \quad$ I3AL 27

$62 S H 11$ I3AL 27

63001 13AL 27

$62001 \quad 13 A L 27$

$58 A \cup 1$ I3AL 27

55 TA1 13AL 27

$56 D A 2 \quad 13 A L 27$

$578 A 2$ I3AL 27

$57 M I 2$ I3AL 27

58BA4 13AL 27

58BA6 I3AL27

$60 \mathrm{CH} 1$ 13AL 27

6IMA2 I3AL 27

$62 \mathrm{CH} 2 \quad 13 \mathrm{AL} 27$

63MI5 I3AL 27

60GO2 13AL27

6IMAI 13AL 27

$60 \mathrm{CHI}$ 13AL 27

$62 \mathrm{CH} 2 \quad 13 \mathrm{AL} 27$

$56 \mathrm{HEI} 13 \mathrm{AL} 27$

56WA1 13AL27

$60 G 02$ 13AL27

$57 B O 1$ 13AL 27

$58 T 02$ I3AL27

6IMAI I3AL27

62BE3 13AL 27

55RE 1 13AL 27

$61 N O 1$ I3AL 27

62LAI 13AL27

62OP I I3AL27

63VA5 13AL27

64VAI 13AL 27

64VA2 13AL27

$63 C V 1$ 13AL 28

$G, P$ SPC THR-70

$G, P \quad R L Y$ THR 65

$G, P \quad R L X \quad 15,18$

G,P SPC 2552

$G, P \quad A B X \quad 1221$

$G, P$ RLY 1135

$G, P \quad S P C \quad 18$

$G, P \quad A B X \quad 15,18$

$G, P \quad S F C \quad 10 \quad 24$

$G, P \quad A B X \quad 10 \quad 24$

$G, P \quad A B Y \quad 10 \quad 22$

E,P $\quad S P C \quad 1729$

$G, 2 P \quad A B X \quad 20 \quad 63$

$G, X P$

$G, X P$

$G, X P$

$G, X P$

$G, X P$

$G, X P$

$G, \times P$

$G, X P$

$G, X P$

$G, X P$

$G, P D$

$G, O$

$G, \times D$

$G, \times D$

$G, T$

$G, T$

G, HE3

$G, A$

$G, A$

$G, A$

$G, A$

$G, N I 7$

$P, G$

$P, G$

$P, G$

$P, G$

$P, G$

$P, G$

$A B X$

$58-280$

$S P C$

8-3I

RLX $\quad 8 \quad 40$

SPC $23 \quad 85$

RLX $24 \quad 90$

NOX $8 \quad 21$

RLY $23 \quad 35$

1222

ABY

30260

RLY 2035

$\begin{array}{lll}R L X & 32 & 90\end{array}$

RLY $32 \quad 35$

$A B X$

$A B X$

$10-31$

RLY 1935

NOX II

NOX 9

$N, G$

$A B X$
IN,OUT MIN-MAX TYPE MIN-MAX

G,N2P ABX 30260 C 30-260 ACT-I

G,4N4P ABX THR $240 \quad$ C120-240 ACT-I

C 70

C 65

EMU-D

[ 15,18

C $18-52$

C $12-21$

C 35

D 18

D 15,18

C 24

C 24

C 22

D $18-30$

C 20-63

C 280

$S P C$ II 70 C 70

$8 \quad 85$

C 85

C 31

C -40 MAG-D

c 85

C 90

C 21

C 35

C 22

SCI- -16

$T E L-D \quad 15-70$

ION-I

$M G P-D$

EMU-D

$S C I-D$

EMU-D

SCI I I

EMU-D

MAG-D 3-15

ACT - I

TEL-D $50-75$

EMU-D 2-14

TEL-D

C $30-260$

TEL-I $15-30$

SCI-D 1-10

MAG-D 15-30

SCI-D 4-

C 35

C 90

C 35

RLY I8-3I C 31

RLY 18-31 C 31

$A C T-I$

MGP-D 3-10

TEL-I 15-30

MAG-D 15-30

$$
30260
$$

c 30

ACT - I

C 31

C 22

c 35

SPC I6 30 C 30

$A C T-I$

EMU-D

EMU - I

$M G P-D$

SCD-D

ABI THR 400

$\begin{array}{rr}\text { C } & 80 \\ 0 & 1\end{array}$

D 3

D $0-1$

NA I -D

NAI -D

RLY 9 IO D $1-2$

NAI - D
NAI -D

NAI -D

D $2-3$

TEL-D
ANG REMARKS

4PI ALSO G,PD, G,HE 3 4PI

DST

* Rel to CU63(G,N)

Al

90

4PI

DST

DST

DST

DST

DST

DST VIRTUAL PHOTONS

4PI

$175 \%$

DST ABY

DST *

DST * SPECTRUM

* QUASI-DEUTERON

90 REL TO DEUTERONS

DST

DST REL TO DEUTERONS

DST

4PI ALSO G,N2P, G,HE3

DST

90 REL TO PROTONS

DST REL TO PROTONS

* REL TO NEUTRONS

4PI * REL TO NEUTRONS

4PI ALSO $G, N 2 P, G, P D$

OST * SPC

4PI

9-14 DST

6-12 DST ASYMMETRY SPECTRUM 4PI

J-PI, WIDTH

DST J-PI

DST J-PI

DST SEPARATED ISOTOPES

DST J-PI

$J-P I$

$4 P I$ 


\begin{tabular}{|c|c|c|c|c|c|c|c|c|}
\hline A & ABUND. (1) & SE & TION & ENERGIES & (MEV) & & & \\
\hline & $\begin{array}{r}G, N \\
17.2\end{array}$ & $\begin{array}{r}G, P \\
11.6\end{array}$ & $\begin{array}{r}G, T \\
27.5\end{array}$ & $\begin{array}{l}\mathrm{G}, \mathrm{HE} 3 \\
23.2\end{array}$ & $\begin{array}{r}G, A \\
10.0\end{array}$ & $\begin{array}{l}G, 2 N \\
30.5\end{array}$ & $\begin{array}{l}G, N P \\
24.7\end{array}$ & $\begin{array}{l}G, 2 P \\
19.9\end{array}$ \\
\hline & 4.70 & 12.3 & 24.6 & 20.6 & 11.1 & 25.7 & 20.1 & 21.9 \\
\hline & 3.09 & 13.5 & 22.2 & $24 \cdot 8$ & 10.7 & 19.1 & 23.0 & 24.0 \\
\hline
\end{tabular}

(1) ABUNDANCE DEPFNDS ON SOURCE

\begin{tabular}{|c|c|c|c|c|c|c|c|c|c|c|}
\hline \multirow[t]{2}{*}{$F$} & \multirow{2}{*}{$\begin{array}{c}\text { NUCL IDE } \\
Z\end{array}$} & \multirow{2}{*}{$\begin{array}{l}\text { REACT ION } \\
\text { IN, OUT }\end{array}$} & \multirow[t]{2}{*}{ RES } & \multirow{2}{*}{\multicolumn{2}{|c|}{ EXCIT }} & \multirow{2}{*}{$\begin{array}{l}\text { SOURCE } \\
\text { MIN-MAX }\end{array}$} & \multicolumn{2}{|c|}{ DETECTOR } & \multirow[t]{2}{*}{ ANG } & \multirow[t]{2}{*}{ REMARKS } \\
\hline & & & & & & & TYPE N & IIN-MAX & & \\
\hline 590U1 & 14 SI & $G, M U-T$ & $A B X$ & 15 & 25 & C 29,30 & $M G C-D$ & & & \\
\hline ORE 1 & 14 SI & $G, G$ & $A B X$ & 7 & & D & NAI $-D$ & & 90 & \\
\hline 61SU1 & 14 S I & $G, G$ & RLY & 11 & & $6-13$ & NAI I D & & 120 & \\
\hline 61TO1 & 14 SI & $G, G$ & $A B X$ & 4 & 14 & $5-14$ & NAI - D & & 120 & \\
\hline 2SE 1 & 14 S I & $G, G$ & NOX & 11 & & C 16 & NAI $-D$ & & 90 & \\
\hline $64 \mathrm{GO3}$ & $145 \mathrm{I}$ & $E, E /$ & $A B X$ & 12 & & D $40-70$ & $M A G-D$ & & 180 & FMF \\
\hline $63 \mathrm{BO} 1$ & 14 S I & $G, N$ & $A B X$ & 15 & 25 & C $10-30$ & BF 3-I & & 4PI & \\
\hline $63 \mathrm{SAl}$ & $14 \mathrm{SI}$ & $G, N$ & $A B X$ & 10 & 24 & C $10-24$ & $B F 3-I$ & & $4 \mathrm{PI}$ & \\
\hline 63003 & $14 S I$ & $G, \times N$ & $A B X$ & 13 & 80 & C $13-80$ & $B F 3-I$ & & $4 P I$ & \\
\hline 58EMI & $14 S I$ & $G, P$ & NOX & THR & 30 & C 30 & $E M U-D$ & & DST & \\
\hline $625 \mathrm{HIl}$ & $14 \mathrm{SI}$ & $G, P$ & $A B X$ & THR & 24 & CTHR-24 & $S C I-I$ & $2-$ & DST & \\
\hline $64 \mathrm{MAl}$ & $14 \mathrm{SI}$ & $G, P$ & $A B X$ & 17 & 22 & D $17-22$ & $S C D-D$ & & $4 \mathrm{PI}$ & BRANCHING RATIOS \\
\hline 64 UL1 & $14 S I$ & $G, P$ & $S P C$ & 14. & -20 & C $17-20$ & $S C D-D$ & $1-9$ & 4PI & SCD TARGET \\
\hline $60 \mathrm{CHI}$ & $14 \mathrm{SI}$ & $G, X P$ & RLX & THR & 90 & C 90 & TEL-I & $15-30$ & 90 & REL TO DEUTERONS \\
\hline $61 \mathrm{SH} 4$ & $14 \mathrm{SI}$ & $G, X P$ & $S P C$ & THR & 24 & C 24 & $E M U-D$ & $2-13$ & DST & ABSOLUTE YIELD \\
\hline $61 \mathrm{SH} 5$ & $14 S \mathrm{I}$ & $G, X P$ & SPC & 14 & 24 & C 24 & EMU-D & $2-12$ & & \\
\hline $64 \mathrm{LO} 2$ & 14 S I & $G, X P$ & SPC & THR - & -21 & C 21 & $S C I-D$ & $2-10$ & $4 \mathrm{PI}$ & SCD TARGET \\
\hline 64UL2 2 & 14 S I & $G, \times P$ & SPC & THR & 30 & C $15-30$ & $S C D-D$ & $2-18$ & 4PI & \\
\hline OCHI & $14 \mathrm{SI}$ & $G, \times D$ & RLX & THR & 90 & C 90 & TEL - I & $15-30$ & 90 & REL TO PROTONS \\
\hline $60 \mathrm{CHI}$ & $14 \mathrm{SI}$ & $G, \times T$ & RLY & THR. & 90 & C 90 & TEL-I & $15-30$ & 90 & REL TO DEUTERONS \\
\hline 64MA 1 & $14 \mathrm{SI}$ & $G, A$ & $A B X$ & 17 & -22 & D $17-22$ & $S C D-D$ & & $4 P I$ & BRANCHING RATIOS \\
\hline 64 UL 1 & 14 SI & $G, A$ & SPC & 14. & -20 & C $17-20$ & $S C D-D$ & & $4 P I$ & SCD TARGET \\
\hline 60TO1 & 14 SI 28 & $G, G$ & RLY & THR & 13 & C 13 & NAI - D & & & THRESHOLD \\
\hline $62 \mathrm{BO} 6$ & 14 S I 28 & $G, G$ & LFT & 2 & & C $0-4$ & NAI $-D$ & & 110 & $J-P I, W I D T H$ \\
\hline $64 \mathrm{BO}$ & $14 S$ I 28 & $G, G$ & LFT & 1 & 3 & $1-3$ & NAI I D & & 100 & $A B I$ \\
\hline 60BA4 & 14 SI 28 & $E, E /$ & $S P C$ & 0 & 30 & D 43 & $M A G-D$ & $13-43$ & 160 & $A B I$ \\
\hline 62ED 1 & 14 SI 28 & $E, E /$ & $A B I$ & 0 & 12 & D 42 & TEL-D & & 180 & G-WDTH \\
\hline $61 \mathrm{BE} 1$ & 14 SI 28 & $G, N$ & RLY & THR & & CTHR & $A C T-I$ & & $4 P I$ & THRESHOLD \\
\hline 63 CA 1 & $14 S I 28$ & $G, N$ & $A B X$ & 17 & 30 & D $15-30$ & BF 3-I & & $4 P I$ & \\
\hline 60 WA2 & 145 I 28 & $G, 4 N 5 P$ & $A B X$ & THR & 240 & $C 240$ & $A C T-I$ & & $4 P I$ & \\
\hline $61 \mathrm{SH} 1$ & 14 S I 28 & $G, P$ & SPC & 14 & 24 & C 24 & EMU-D & $2-12$ & & \\
\hline $62 \mathrm{BI} 1$ & 14 SI 28 & $G, P$ & $S P C$ & 18 & & D 18 & $S C D-D$ & $4-9$ & DST & \\
\hline $62 \mathrm{BII}$ & 14 S I 28 & $G, A$ & SPC & 18 & & D 18 & $S C D-D$ & $4-9$ & DST & \\
\hline $60 \mathrm{KI} 3$ & 14 S I 28 & $P, G$ & $R L X$ & 19 & 26 & $8-15$ & NAI $-D$ & & 90 & \\
\hline $61 \mathrm{GOl}$ & 14 S I 28 & $P, G$ & RLY & 15 & 22 & $3-11$ & NAI I D & & DST & \\
\hline $61 \mathrm{KII}$ & $14 S$ I 28 & $P, G$ & $A B X$ & 7 & 15 & $7-15$ & $N A I-D$ & & 90 & \\
\hline 63AN2 & $14 S I 28$ & $P, G$ & NOX & 13 & & $1-3$ & $N A I-D$ & & 90 & \\
\hline 63511 & 14 S I 28 & $P, G$ & NOX & 12 & & D & NAI I D & & DST & $J-P I$ \\
\hline $64 A L 3$ & 14 SI 28 & $P, G$ & $A B X$ & 15 & 24 & $4-13$ & NAI I D & & DST & \\
\hline $64 \mathrm{RA} 1$ & 14 S I 28 & $P, G$ & RLX & 16. & -18 & $5-7$ & NAI -D & & 90 & FLUCTUATIONS \\
\hline $625 M 1$ & 14S I 28 & $A, G$ & $A B Y$ & 11 & 13 & $1-3$ & NAI I D & $8-13$ & DST & J-PI, SEP I SOTOP \\
\hline 64WE 1 & 14 S I 28 & $A, G$ & NOX & 12 & -14 & $3-5$ & NAI $-D$ & & DST & WIDTHS \\
\hline $\begin{array}{l}62806 \\
6] B F 1\end{array}$ & 14SI 29 & $G, G$ & LFT & & & C $0-4$ & NAI I D & & DST & J-PI, WIDTHS \\
\hline & 145129 & $G, N$ & RLY & & & CTHR & $A C T-I$ & & $4 P I$ & THRESHOLD \\
\hline $1 B E 1$ & 145130 & $G, N$ & RLY & THR & & CTHR & $A C T-I$ & & $4 P I$ & THRESHOLD \\
\hline
\end{tabular}




\begin{tabular}{|c|c|c|c|c|c|c|c|c|c|}
\hline A & ABUND. & & SEF & ATION & ENERGIES & (MEV) & & & \\
\hline 31 & 100.00 & $\begin{array}{r}G, N \\
12.3\end{array}$ & $\begin{array}{l}G, P \\
7.3\end{array}$ & $\begin{array}{r}G, T \\
17.9\end{array}$ & $\begin{array}{l}\text { G.HE3 } \\
22.5\end{array}$ & $\begin{array}{l}G, A \\
9.7\end{array}$ & $\begin{array}{l}G, 2 N \\
23.6\end{array}$ & $\begin{array}{l}G, N P \\
17.9\end{array}$ & $\begin{array}{l}G, 2 P \\
20.8\end{array}$ \\
\hline
\end{tabular}

REF NUCLIDE REACTION RES EXCIT SOURCE DETECTOR ANG REMARKS

GONEI A IN,OUT MIN-MAX TYPE MIN-MAX

GONEI I5P 29 P,G NOX 455 D $1-3$ NAI-D N DST J-PI, WIDTH

GIVA3 I5P 29 P,G NOX 35 D $0-2$ NAI-D DST J-PI, WIDTHS

6IVA2 $15 P \quad 30$

62AN2 $15 P 30$

63VA2 15P 30

$64 E J 1 \quad 15 P \quad 30$

$P, G \quad N O X \quad 7 \quad D \quad 1 \quad$ NAI -D

$P, G \quad N O X \quad 7 \quad 8 \quad D \quad 1-2 \quad$ NAI-D

$P, G$ NOX 7 D 12 NAI-D

$P, G$ NOX 7 D 1 NAI

DST J-PI

$90 \mathrm{~J}-\mathrm{PI}$

G,MU-T ABX $10 \quad 30 \quad C 29,30 \quad M G C-D$

$590 \cup 1 \quad 15 P \quad 31$

$61803 \quad 15 P \quad 31$

$60803 \quad 15 P 31$

$62 B 06 \quad 15 P \quad 31$

64BO1 15P 31

63BAl 15P 31

63LO2 15P 31

$55 B A 2 \quad 15 P \quad 31$

$56 S C 1 \quad 15 P \quad 31$

$58 \mathrm{CH} 2 \quad 15 \mathrm{P} 31$

60GE3 15P 31

$61 S A 1$ 15P 31

$G, M U-T$

$G, G$ LFT 1

10-20 THR $-\mathrm{I}$

GPG C $0-2$ NAI

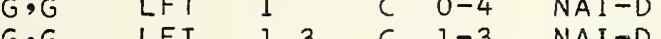

$G, G \quad$ LFT 1 1 3 C $1-3$

E,E/ SPC 0 15

D 42

MAG-D 27-42

E,E/ FMF 16 DI30,180 MAG-D

$G, N \quad$ RLY $12 \quad 13 \quad C \quad 12-13$ ACT-I

$G, N \quad R L Y \quad 12-22 \quad C 22 \quad A C T-I$

$G, N$ RLY THR CTHR BF 3-I

$G, N$ NOX THR CTHR ACT-I

$G, N \quad R L Y \quad 1214 \quad C \quad 12-14 \quad$ ACT-I

G,N SPC 1030 C 30 EMU-D

$G, N \quad A B X \quad 1224 \quad C 24 \quad B F 3-I$

$G, N \quad A B X \quad 1328$ C $10-30$ BF3-I

$G, \times N$

$\begin{array}{lllllll}G, X N & A B X & 13 & 80 & C & 80 & B F 3-1 \\ G, X N & A B X & 12 & 24 & C & 12-24 & A C T-I\end{array}$

CTHR

$62 M U 2$ 15P 31

$57 B A 3 \quad 15 P \quad 31$

$63 C 03 \quad 15 P \quad 31$

$63 M C 3 \quad 15 P \quad 31$

$590 C 1 \quad 15 P \quad 31$

60WA2 15P 31

$625 \mathrm{SH} 215 \mathrm{P} 31$

$590 C 1 \quad 15 P \quad 31$

$615 H^{4} \quad 15 P \quad 31$

$64 I S 1$ 15P 31

$60602 \quad 15 P \quad 31$

62 TU1 15P 31

G,N2P RLI THR-100 CTHR-100 ACT-I

G,6NGP ABX THR $240 \quad$ C240 ACT-I

$G, P \quad S P C \quad 10 \quad 19 \quad C 19$ EMU-D

G,2P RLI THR-100 CTHR-100 ACT-I

$G, X P$ SPC 924 C 24 EMU-D

G,XP ABX THR-32 C 15-32 SCI-D

G,NA24 ABX 30260 C 30-260 ACT-I

$P, G \quad N O X \quad 899$ D $1-2$ EMU-D

DST J-PI, WIDTHS

64TU1 15P 31

$\$ P, G$ NOX 10 D 3 EMU-D

4 PI $*$

$100 \mathrm{~J}-\mathrm{PII}_{1}$

$110 \mathrm{~J}-P I$, WIDTH

$100 \mathrm{ABI}$

$180 \mathrm{ABX}$

DST

4PI THRESHOLD

4PI *

4PI THRESHOLD

4PI THRESHOLD

4PI THRESHOLD

$2-13$ DST

4P I

$4 P I$

4PI

4PI

4PI REL TO $G, N$

4PI

2-12 4PI

4PI REL TO G, N

2-14 DST ABSOLUTE YIELD

$0-3$

4PI INCLUDES G,HE 4NZP

DST J-PI

$90 \mathrm{~J}-\mathrm{PI}, \mathrm{POL}$ OF $G$ 


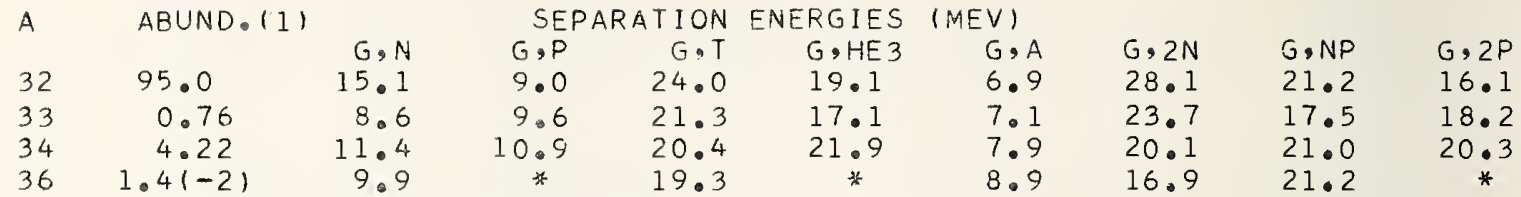

(1) ABUNDANCE DEPENDS ON SOURCE

\begin{tabular}{|c|c|c|c|c|c|c|c|c|c|c|c|}
\hline \multirow[t]{2}{*}{ REF } & \multicolumn{2}{|c|}{ NUCL IDE } & \multirow{2}{*}{$\begin{array}{l}\text { REACT ION } \\
\text { IN, OUT }\end{array}$} & \multirow[t]{2}{*}{ RES } & \multirow{2}{*}{\multicolumn{2}{|c|}{ EXCIT }} & \multirow{2}{*}{$\begin{array}{l}\text { SOURCE } \\
\text { MIN-MAX }\end{array}$} & \multicolumn{2}{|c|}{ DETECTOR } & \multirow[t]{2}{*}{ ANG } & \multirow[t]{2}{*}{ REMARKS } \\
\hline & & & & & & & & TYPE & MIN-MAX & & \\
\hline 59DUI & $16 S$ & & $G, M U-T$ & $A B X$ & 11 & 30 & C 29,30 & $M G C-D$ & & & \\
\hline $59 \mathrm{KO} 1$ & $16 S$ & & $G, M U-T$ & $A B X$ & 12 & 30 & C 31 & $N A I-D$ & & & \\
\hline $56 \mathrm{FUI}$ & $16 S$ & & $G, G$ & $A B X$ & 4 & 40 & C $4-40$ & NAI-D & & 120 & \\
\hline 6ORE 1 & 165 & & $G, G$ & $A B X$ & 7 & & D & NAI I-D & & 90 & \\
\hline 61T01 & 165 & & $G, G$ & $A B X$ & 4 & 13 & $5-13$ & NAI-D & & 120 & \\
\hline $64 \mathrm{BO} 1$ & $16 S$ & & $G, G$ & LFT & 1 & 3 & $1-3$ & NAI -D & & 100 & $A B I$ \\
\hline $63 \mathrm{BOI}$ & 165 & & $G \cap N$ & $A B X$ & 12 & 28 & C $10-30$ & BF 3-I & & 4PI & \\
\hline $63 \mathrm{CO} 3$ & $16 S$ & & $G, \times N$ & $A B X$ & 14 & 80 & C $\quad 0-80$ & BF 3-I & & $4 \mathrm{PI}$ & \\
\hline $63 M U 2$ & $16 S$ & & $G, \times N$ & $A B X$ & 15 & 22 & C $15-22$ & $S C I-D$ & $3-9$ & 90 & SPECTRUM \\
\hline $64 \mathrm{FI} 1$ & $16 \mathrm{~S}$ & & $G, \times N$ & SPC & THR & 25 & C 23,25 & TOF-D & & 70 & \\
\hline $55 \mathrm{RII}$ & 165 & & $G, N P$ & RLY & THR- & -65 & C 65 & $\mathrm{CCH}-\mathrm{I}$ & & & * REL TO DEUTERONS \\
\hline $55 R I 1$ & $16 S$ & & $G, N D$ & RLY & THR- & .65 & C 65 & $\mathrm{CCH}-\mathrm{I}$ & & & * REL TO PROTONS \\
\hline $61 \mathrm{FO}$ & 165 & & $G, P$ & SPC & 10 & 30 & C 30 & EMU-D & $2-17$ & DST & \\
\hline $62 \mathrm{SH} 11$ & $16 \mathrm{~S}$ & & $G, P$ & $A B X$ & 9 & 24 & C $9-24$ & $S C I-1$ & & DST & \\
\hline $60 \mathrm{CHI}$ & 165 & & $G, \times P$ & $R L X$ & THR & 90 & C 90 & $T E L-I$ & $15-30$ & 90 & REL TO DEUTERONS \\
\hline $61 M A 2$ & 165 & & $G, X P$ & NOX & 9 & 21 & C 21 & $S C I-D$ & $1-10$ & DST & \\
\hline $63 \mathrm{SH} 4$ & $16 S$ & & $G, X P$ & SPC & 11 & 24 & C 24 & EMU-D & $2-15$ & & \\
\hline 64151 & 165 & & $G, X P$ & $A B X$ & THR- & -32 & c $15-32$ & $S C I-D$ & $0-3$ & & \\
\hline $6 \mathrm{CCH} 1$ & $16 S$ & & $G, X D$ & RLX & THR & so & C 90 & $T E L-I$ & $15-30$ & 90 & REL TO PROTONS \\
\hline $60 \mathrm{CH} 1$ & 165 & & $G, \times T$ & RLY & THR & 90 & C 90 & TEL-I & $15-30$ & 90 & REL TO DEUTERONS \\
\hline $62 B 06$ & 165 & 32 & $G, G$ & LFT & 0 & 4 & $0-4$ & MAI-D & & 110 & $J \rightarrow P I$ \\
\hline $61 B I 1$ & 165 & 32 & $E, E /$ & FMF & 0 & -4 & D150 & $M A G-D$ & & 135 & MULTIPOLARITY \\
\hline 63BA 1 & 165 & 32 & $E, E /$ & SPC & 0 & 22 & D 42 & $M A G-D$ & $17-42$ & 180 & G-WIDTH \\
\hline $64 \mathrm{LO} 1$ & $16 \mathrm{~S}$ & 32 & $E, E /$ & FMF & 0 & 9 & D120-180 & $M A G-D$ & & DST & $J-P I$ \\
\hline $59 \mathrm{FAl}$ & 165 & 32 & $\mathrm{G}, \mathrm{N}$ & $A B X$ & 20 & 30 & C 30 & THR - I & $5-$ & & \\
\hline $59 \mathrm{FAl}$ & 165 & 32 & $G, N$ & $A B X$ & 16 & 30 & C 30 & $A C T-I$ & & 4PI & \\
\hline 60FE]. & 165 & 32 & $G, N$ & $A B X$ & 15 & 30 & C 31 & $A C T-I$ & & $4 P I$ & \\
\hline $62 \mathrm{KUI}$ & $16 S$ & 32 & $G, N$ & $A B X$ & 15 & 25 & C $12-27$ & $A C T-I$ & & $4 P I$ & \\
\hline $62 M \cup 2$ & 165 & 32 & $G, N$ & $A B X$ & 15 & 22 & C $12-24$ & BF 3-I & & $4 P I$ & \\
\hline 60F 다 & 165 & 32 & $G, \times N$ & $A B X$ & 1.2 & 31 & C 31 & MOD-I & & 4PI & \\
\hline $60 \mathrm{FE} 1$ & 165 & 32 & $G, \times N$ & $A B X$ & 20 & 31 & C 31 & THR-I & $5-$ & & \\
\hline 62303 & 165 & 32 & $G, X N$ & $A B X$ & 16 & 90 & C $30-90$ & BF 3-I & & $4 \mathrm{PI}$ & \\
\hline $55 D E 1$ & $16 \mathrm{~S}$ & 32 & $G, N P$ & $A B X$ & 18 & 22 & C $18=22$ & $A C T-I$ & & 4PI & \\
\hline $58 G 03$ & $16 \mathrm{~s}$ & 32 & $G, N P$ & RLY & 21 & 22 & C $16-22$ & $A C T-I$ & & $4 P I$ & REACTION UNCERTAIN \\
\hline 59FA1 & $16 S$ & 32 & $G, N P$ & $A B X$ & 21 & 30 & C 30 & $A C T-I$ & & $4 P I$ & \\
\hline $60 F E 1$ & 165 & 32 & $G, N P$ & $A B X$ & 21 & 31 & C 31 & $A C T-I$ & & 4PI & \\
\hline $62 \mathrm{BO} 3$ & 165 & 32 & $G, N P$ & $A B X$ & 20 & 90 & C $30-90$ & $A C T-I$ & & $4 \mathrm{PI}$ & \\
\hline 6OWA2 & 165 & 32 & $G, 6 N 7 P$ & $A B X$ & THR & 240 & $C 240$ & $A C T-I$ & & 4PI & \\
\hline $55 \mathrm{VII}$ & 165 & 32 & $G, D$ & $A B X$ & $19-$ & -22 & C $17-22$ & $A C T-I$ & & 4PI & INCLUDES G,NP \\
\hline 58603 & $16 \mathrm{~S}$ & 32 & $G, D$ & RLY & 19 & 22 & C $16-22$ & $A C T-I$ & & $4 \mathrm{PI}$ & REACTION UNCERTAIN \\
\hline $62 B E 4$ & 165 & 32 & $P, G$ & $A B Y$ & 9 & 10 & D $0-1$ & $N A I-D$ & & DST & $J-P I$ \\
\hline 62NE1 & 165 & 32 & $P, G$ & RLY & 9 & 10 & $0-1$ & NAI-I & & 90 & $J-P I$ \\
\hline $63 \mathrm{CH} 1$ & 165 & 32 & $P, G$ & SPC & 9 & 10 & $0-1$ & $N A I-D$ & & DST & $J-P I$ \\
\hline $63 \mathrm{KI} 2$ & $16 \mathrm{~S}$ & 32 & $P, G$ & $A B X$ & 17 & 22 & $8-14$ & $N A I-I$ & & DST & \\
\hline 63SP1 & 165 & 32 & $P, G$ & NOX & 9 & 10 & $0-1$ & $N A 1-D$ & & DST & $J-P I, G-W D T H$ \\
\hline 64 SM1 & 165 & 32 & $P, G$ & $A B Y$ & 9 & 10 & $0-1$ & $N A I-D$ & & DST & J-PI, WIDTHS \\
\hline $645 M 1$ & 165 & 32 & $A, G$ & $A B Y$ & 9 & 10 & $2-3$ & $N A I-D$ & & DST & $J-P I$, WDTHS \\
\hline 2806 & 165 & 34 & $G, G$ & LFT & 0 & 4 & $0-4$ & NAI-D & & 110 & $J-P I$ \\
\hline
\end{tabular}




\begin{tabular}{|c|c|c|c|c|c|c|c|c|c|}
\hline & ABUND. & & SEP & ATION & ENERGIES & (MEV) & & & \\
\hline 3 & & $\begin{array}{r}G, N \\
12.6\end{array}$ & $\begin{array}{l}G, P \\
6.4\end{array}$ & $\begin{array}{r}G, T \\
18.0\end{array}$ & $\begin{array}{l}\text { G.HE } 3 \\
19.6\end{array}$ & $\begin{array}{l}G, A \\
7.0\end{array}$ & $\begin{array}{l}G, 2 N \\
24.1\end{array}$ & $\begin{array}{l}G, N P \\
17.8\end{array}$ & $\begin{array}{l}G, 2 \\
17 .\end{array}$ \\
\hline & 24.47 & 10.3 & 8.4 & 16.8 & 21.9 & 7.9 & 18.9 & 18.3 & \\
\hline
\end{tabular}

\begin{tabular}{|c|c|c|c|c|c|c|c|c|}
\hline REF & $\begin{array}{r}\text { NUCL IDE } \\
Z \quad A\end{array}$ & $\begin{array}{l}\text { REACT I ON } \\
\text { IN,OUT }\end{array}$ & RES & EXCI T & $\begin{array}{l}\text { SOURCE } \\
\text { MIN-MAX }\end{array}$ & $\begin{array}{c}\text { DETECTOR } \\
\text { TYPE MIN-MAX }\end{array}$ & ANG & REMARKS \\
\hline $57 B A 3$ & $17 \mathrm{CL}$ & $G, \times N$ & & THR & CTHR & & & * \\
\hline 55ER 1 & $17 \mathrm{CL}$ & $G, A$ & $A B X$ & $T H R-32$ & C 32 & EMU-I & & * AGCL EMULSION \\
\hline $62 B 06$ & $17 C L 35$ & $G, G$ & LFT & 04 & $0-4$ & NAI -D & 110 & $J-P I$ \\
\hline $55 \mathrm{BA} 3$ & $17 C L 35$ & $G, N$ & RLY & 1222 & C 22 & $A C T-I$ & 4P I & THRESHOLD \\
\hline $5 \mathrm{BO} 2$ & $17 \mathrm{CL} 35$ & $G, N$ & $A B X$ & $13-21$ & C $13-21$ & $A C T-I$ & $4 \mathrm{PI}$ & \\
\hline 55DE 1 & $17 C L 35$ & $G, N$ & $A B X$ & 1321 & C $13-21$ & $A C T-I$ & 4P I & THRESHOLD \\
\hline $565 \mathrm{Cl}$ & $17 C L 35$ & $G, N$ & $R L Y$ & $12-22$ & C 22 & $A C T-I$ & $4 \mathrm{PI}$ & * \\
\hline $59 \mathrm{FE} 1$ & $17 \mathrm{CL} 35$ & $G, N$ & $A B X$ & 1231 & C 31 & $A C T-I$ & 4P I & \\
\hline $59 \mathrm{FEl}$ & $17 C L 35$ & $G, N$ & $A B X$ & 1631 & C 31 & THR - I & 4P I & \\
\hline $615 A 1$ & $17 C L 35$ & $G, N$ & $R L Y$ & 1214 & C $12-14$ & $A C T-I$ & $4 \mathrm{PI}$ & THRESHOLD \\
\hline $62 K \cup 1$ & $17 C L 35$ & $G, N$ & $A B X$ & 1225 & C 27 & $A \subset T-I$ & 4P I & \\
\hline S9SEI & $17 C L 36$ & $N, G$ & $S P C$ & 9 & c & $N A I-D$ & 90 & G-WIDTH \\
\hline $2 \mathrm{BO} 6$ & $17 C \operatorname{CL} 37$ & $G, G$ & LFT & 04 & $0-4$ & NAI -D & 110 & $J-P I$ \\
\hline OGE 3 & $17 C L 37$ & $G, N$ & NOX & THR & CTHR & BF 3-I & 4P I & THRESHOLD \\
\hline
\end{tabular}

ARGON $Z=18$

\begin{tabular}{|c|c|c|c|c|c|c|c|c|c|}
\hline 4 & ABUND. & & SE & ATION & ENERGIES & (MEV) & & & \\
\hline & 0 & $\begin{array}{r}G, N \\
15.3\end{array}$ & $\begin{array}{l}G, P \\
8.5\end{array}$ & $\begin{array}{r}G, T \\
24.2\end{array}$ & $\begin{array}{l}\text { G.HE3 } \\
18.6\end{array}$ & $\begin{array}{l}G, A \\
6,6\end{array}$ & $\begin{array}{l}G, 2 N \\
28.3\end{array}$ & $\begin{array}{l}G, N P \\
21.2\end{array}$ & $\begin{array}{l}\text { G.2P } \\
14.9\end{array}$ \\
\hline & $\begin{array}{c}6.3(-2) \\
99.6\end{array}$ & $\begin{array}{r}11.8 \\
9.9\end{array}$ & $\begin{array}{l}10.2 \\
12.5\end{array}$ & $\begin{array}{l}20.7 \\
18.2\end{array}$ & $\begin{array}{l}20.8 \\
23.0\end{array}$ & $\begin{array}{l}7.2 \\
6.8\end{array}$ & $\begin{array}{l}20.6 \\
16.4\end{array}$ & $\begin{array}{l}20.6 \\
20.6\end{array}$ & $\begin{array}{l}18.6 \\
22.8\end{array}$ \\
\hline
\end{tabular}

REF NUCLIDE REACTION RES EXCIT SOURCE DETECTOR ANG REMARKS

\begin{tabular}{|c|c|c|c|c|c|c|c|c|c|c|c|}
\hline & $z$ & IN, OUT & & & & IN-MAX & TYPE N & $N-M A X$ & & & \\
\hline $57 \mathrm{KOZ}$ & $18 \mathrm{AR}$ & $G, P$ & & $T H R-90$ & C & 90 & EMU-D & & DST & * 302 & TRACKS \\
\hline 59EM2 & $18 \mathrm{AR}$ & $G, P$ & SPC & $10 \quad 30$ & c & $23-30$ & EMU-D & $2-14$ & 90 & & \\
\hline $61 \mathrm{FII}$ & $18 \mathrm{AR}$ & $G, P$ & RLY & $0 \quad 35$ & C & 35 & $S C I-D$ & & 90 & & \\
\hline $555 P 3$ & $18 \mathrm{AR}$ & $G, X P$ & $S P C$ & THR -23 & C & 23 & & & & * ABS & Y I ELD \\
\hline 59EM2 & $18 \mathrm{AR}$ & $G, A$ & $A B X$ & $6 \quad 12$ & C & $23-30$ & EMU-D & & 90 & & \\
\hline $61 \mathrm{KOl}$ & I8AR & $G, A$ & $S P C$ & 1017 & C & 70 & I ON-D & $3-12$ & 90 & & \\
\hline 64ER 1 & 18 AR 36 & $P, G$ & RLY & $8 \quad 10$ & D & $2-3$ & $N A I-D$ & & DST & J-P I, & WIDTHS \\
\hline 64ER I & 18 AR 36 & $A, G$ & RLY & $8 \quad 10$ & D & $2-3$ & $N A I-D$ & & DST & $J-P I$, & WIDTHS \\
\hline $\begin{array}{l}64 \mathrm{ER} 1 \\
64 \mathrm{PHI}\end{array}$ & $\begin{array}{l}18 A R 38 \\
18 A R 38\end{array}$ & $\begin{array}{l}A, G \\
A, G\end{array}$ & $\begin{array}{l}\text { RLY } \\
\text { RLY }\end{array}$ & $\begin{array}{rl}9 & 10 \\
99 & 11\end{array}$ & $\begin{array}{l}D \\
D\end{array}$ & $\begin{array}{l}2-3 \\
3-4\end{array}$ & $\begin{array}{l}\text { NAI I D } \\
\text { NAI -D }\end{array}$ & & $\begin{array}{l}\text { DST } \\
\text { DST }\end{array}$ & $\begin{array}{l}\text { J-P I, } \\
\text { J-PI, }\end{array}$ & $\begin{array}{l}\text { WIDTHS } \\
\text { WIDTHS }\end{array}$ \\
\hline
\end{tabular}


Ar REF

$\mathrm{K}$
NUCLIDE REACTION RES

$Z$ A IN,OUT

63BAI

59PE 1

6OFAl

$59 F E 1$

$59 B R 1$

$59 P E 1$

60001

$56 \mathrm{KO}$

$58 \mathrm{GU} 1$

59ER I

5OPE 1

60001

62001

$58 \mathrm{IAI}$
$E, E /$

$G, N$

$G, N$

$G, 2 N$

$G, N P$

$G, N P$

$G, N P$

$G, P$

$G, P$

$G, P$

$G, P$

$G, P$

$E, P$

$G, X P$
EXCIT

SPC 024 $A B X \quad 10 \quad 23$ $A B X \quad 13 \quad 49$ $\begin{array}{lll}A B X & 17 & 23\end{array}$ $A B I \quad 21 \quad 34$ $A B \times \quad 1940$ ABI $21 \quad 33$ NOX 1290 SPC $13 \quad 15$ ABI $12 \quad 34$ $A B X \quad 13 \quad 33$ $A B I \quad 12 \quad 33$ SPC $13 \quad 30$ $A B I \quad 1270$
SOURCE

MIN-MAX TYPE MIN-MAX

D 42

C 14-44

C $10-50$

C $14-44$

C 34

C $14-44$

c 30,34

C 90

C 15

C 34

C $14-44$

C 30,34

D 30

C 70
MAG-D 18-42

$A C T-I$

BF 3-I

$A C T-I$

$A C T-I$

ACT - I

ACT-I

$\mathrm{CCH}-\mathrm{I}$

$\mathrm{CCH}-\mathrm{D}$

ACT - I

$A C T-I$

$A C T-I$

$M A G-D$

CCH-D
ANG

REMARKS

180

4PI SYNTHESIS

4PI

4PI SYNTHESIS

4PI

4PI SYNTHESIS

4PI

DST

DST

4P I

4PI SYNTHESIS

4PI

3-16 76 SEPARATED ISOTOPE

$0-14$ DST

POTASSIUM $Z=19$

\begin{tabular}{|c|c|c|c|c|c|c|c|c|c|}
\hline A & AEUND. & & SE & ATION & ENERGIES & (MEV) & & & \\
\hline & & $G, N$ & $G, P$ & G, T & G, HE 3 & $G, A$ & $\mathrm{G} \cdot 2 \mathrm{~N}$ & $G, N P$ & $G, 2 P$ \\
\hline 39 & 93. & 13.1 & 6.4 & 18.5 & 19.2 & $7 \cdot 2$ & 25.1 & $18 \cdot 2$ & b. \\
\hline 40 & $1 \cdot 2(-2)$ & 7.8 & 7.6 & 17.5 & 16.7 & 6.4 & 20.9 & 14.2 & $18 \cdot 3$ \\
\hline 41 & 6.88 & 10.1 & 7.8 & 15.8 & 20.7 & 6.2 & 17.9 & 17.7 & 0 \\
\hline
\end{tabular}

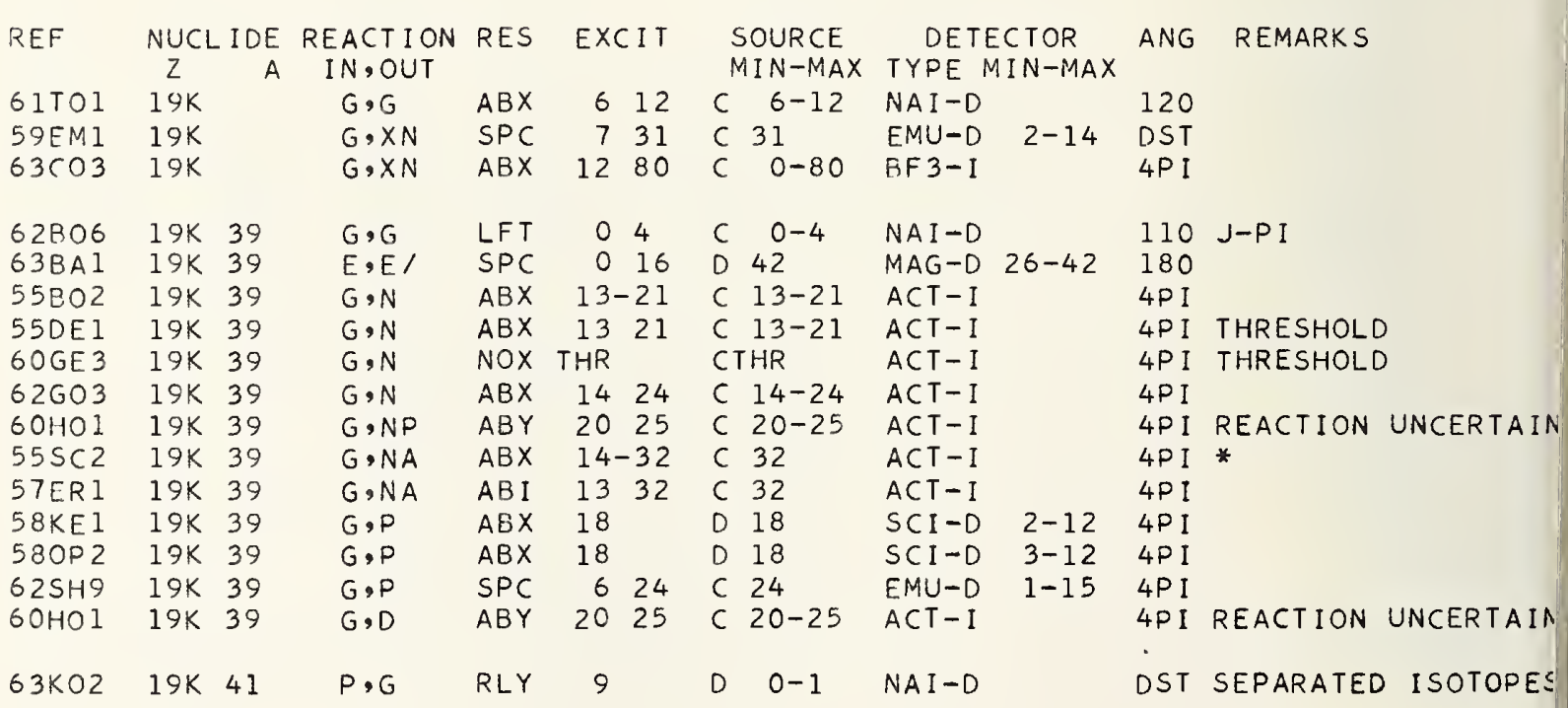




\begin{tabular}{|c|c|c|c|c|c|c|c|c|c|}
\hline A & ABUND. & & SE & ATION & ENERGIES & (MEV) & & & \\
\hline & & $G, N$ & $G, P$ & $G, T$ & G, HE 3 & $G, A$ & $G, 2 N$ & $G, N P$ & $G, 2 P$ \\
\hline 40 & 96.97 & 15.7 & 8.3 & 25.0 & 18.8 & 7.0 & 29.3 & 21.5 & 14.7 \\
\hline 42 & 0.64 & 11.5 & 10.3 & 19.7 & 20.2 & 6.2 & 19.8 & 20.4 & 18.1 \\
\hline 43 & 0.15 & 7.9 & 10.7 & 19.8 & 18.3 & 7.6 & 19.4 & 18.2 & 19.9 \\
\hline 44 & 2.06 & 11.1 & 12.2 & 20.9 & $23 \cdot 3$ & 8.8 & 19.1 & 21.8 & 21.6 \\
\hline 46 & $3 \cdot 3(-3)$ & 10.4 & 13.8 & 21.5 & * & 11.1 & 17.8 & 23.2 & * \\
\hline 48 & $0.19^{\circ}$ & 9.9 & 15.3 & 22.5 & 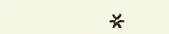 & * & 17.2 & 24. & * \\
\hline
\end{tabular}

REF NUCLIDE REACTION RES EXCIT $Z$ A IN,OUT

59DUI 20CA G,MU-T ABX 1130

$56 F U 1$ 20CA $G, G \quad A B X \quad 440$

$610 E 4$ 2OCA

61TO1 20CA

60KU2 20CA

$59 A G 1$ 20CA

$59 E M 120 C A$

$63 C 03$ 20CA

$64 \mathrm{FII} 20 \mathrm{CA}$

$575 C 1 \quad 20 \mathrm{CA}$

$59 \mathrm{KO} 220 \mathrm{CA}$

$64 I S 2$ 20CA

64RA2 20CA

63MI 5 20CA

$64 \mathrm{HAI} 20 \mathrm{CA}$

64SII 20CA

6IECI $20 C A 40$

62BO6 20CA40

6IPEI $20 C A 40$

62EDI 20CA40

63BAI 2OCA 40

$633 \mathrm{LI}$ 20CA40

$64 \mathrm{HOI} 20 \mathrm{CA} 40$

62MI2 20CA4O

$63 \mathrm{MI} 2$ 20CA4O

6OFEI 2OCA 40

$60 \mathrm{FEI} 20 \mathrm{CA} 40$

$60 \mathrm{LI} 220 \mathrm{CA} 4 \mathrm{O}$

64BAI 20CA4O

$58 \mathrm{HOI} 20 \mathrm{CA} 40$

6OFEI 20CA4O

620R1 20CA40

$62 \mathrm{JOI} 20 \mathrm{CA} 40$

$62 \mathrm{SHI} 220 \mathrm{CA} 40$

$62 \mathrm{CH} 220 \mathrm{CA} 40$

$62 \mathrm{CH} 2 \quad 20 \mathrm{CA} 40$

6IPOI $20 C A 40$

62RAI 20CA40

64TAI 20CA40

$6351220 C A 42$

$58 B R 1 \quad 20 C A 44$

$G, G \quad A B X \quad 1532$

$G, G \quad A B X \quad 6 \quad 12$

$G, N$ RLY I5 90

$G, X N$ SPC THR-30

$G, X N \quad S P C \quad 731$

$G, X N \quad A B X \quad 1580$

G, 3N3P ABX THR-70

$G, P$ SPC THR-85

$G, P \quad A B X \quad 14-32$

$G, P \quad A B X \quad 14 \quad 27$

$G, X P \quad A B Y \quad 6 \quad 22$

$P, G \quad A B X \quad 14 \quad 23$

$P, G \quad N O X \quad 910$

$G, G \quad$ NOX 10

G, G. LFT 04

$E, E / \quad A B X \quad 1126$

$E, E /$

$E, E /$

$E, E /$

$E, E /$

$G, N$

$G, N$

$G, \times N$

$G, \times N$

$G, \times N$

$G, X N$

$G, N P$

$G, N P$

$G, P$

$G, P$

$G, P$

$G, X P$

$G, \times D$

$P, G$

$P, G$

$P, G$

NOX

$0-$

023

0 11

10-17

$\begin{array}{ll}15 & 23\end{array}$

$A B X \quad 15 \quad 26$

$A B X \quad 12 \quad 31$

$A B X$ I8 31

ABI THR 34

$A B X \quad I 5 \quad 30$

$A B X \quad 26 \quad 32$

$A B X \quad 2131$

SPC 822

$$
\text { SPC } 821
$$

$S P C \quad 1021$

$\begin{array}{lll}R L Y & 8 & 35\end{array}$

$\begin{array}{lll}R L Y & 19 & 35\end{array}$

RLY 910
$G, X N$ SPC THR 32

SOURCE

MIN-MAX TYPE MIN-MAX

C 29,30 MGC-D

C 4-40 NAI-D

C 32 NAI-D

C 6-12 NAI-D

C 90 TEL-I 10-

c 30

C 31

C $0-80$

c 25,32

C 70

c 85

C $14-32$

C 30

C 22

D $6-15$ SCI-D

D $1-2$ NAI-D

D 10 NAI-D

$C \quad 0-4 \quad N A I-D$

DI20-180 MAG-D

D 42 TEL-D

D 42

MAG-D 18-42

D120-220 MAG-D169-179

D 80-200 MAG-D

D $15-25$ BF 3-I

C $15-30$ BF 3-I

c 31

c 31

$A C T-I$

THR-I 5-

C 34

C $15-30$

NAI - I

$A C T-1$

C 26-32 ACT-I

C $31 \quad$ ACT -1

C 22

c 20,21

c 21

c 35

C 35

D $1-2$

EMU-D

EMU-D

EMU-D

$M A G-D$

$M A G-D$

NAI I D

D $1-2$ NAI -D

D 9-14 NAI-D

$P, G \quad R L Y \quad I 112$ D $1-2$ NAI-D

ACT-I

$1-13$

$5-$

$4-$

$2-14$

* surface effects

$4 P I$

70

*

* 7000 TRACKS

DST SPC

DST INCLUDES G,NP

DST

90 DETAILED BALANCE

DST J-PI

DST J-PI, G-WIDTH

$110 \mathrm{~J}-\mathrm{PI}$

DST

160 NO EXCITATION OBS

$180 \mathrm{~J}-\mathrm{PI}$

100

4PI

$4 P I$

4P I

$5-$

$G, P \quad A B I \quad 12 \quad 31 \quad C \quad 31$

$1-13$

4PI

4PI THRESHOLD

4PI

$2-13$

DST REL TO DEUTERONS

DST REL TO PROTONS

DST J-PI, G-WIDTH

100

SEPARATED ISOTOPES

4PI 


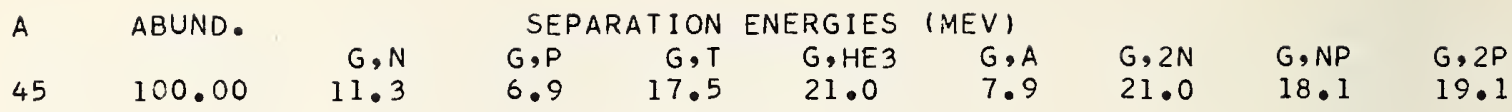

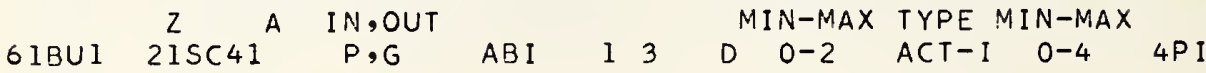

TITANIUM $Z=22$

\begin{tabular}{|c|c|c|c|c|c|c|c|c|c|}
\hline A & ABUND. & & SE & ATION & ENERGIES & (MEV) & & & \\
\hline 6 & 7.93 & $\begin{array}{r}G, N \\
13.1\end{array}$ & $\begin{array}{r}G, P \\
10.4\end{array}$ & $\begin{array}{r}G, T \\
22.9\end{array}$ & $\begin{array}{l}\text { G, HE3 } \\
20.7\end{array}$ & $\begin{array}{l}G, A \\
8.0\end{array}$ & $\begin{array}{l}G, 2 N \\
22.6\end{array}$ & $\begin{array}{l}G, N P \\
21.7\end{array}$ & $\begin{array}{l}G, 2 P \\
17.2\end{array}$ \\
\hline+7 & $\begin{array}{r}7.28 \\
73.94\end{array}$ & $\begin{array}{r}8.9 \\
11.6\end{array}$ & $\begin{array}{l}10.5 \\
11.4\end{array}$ & $\begin{array}{l}22.0 \\
22.4\end{array}$ & $\begin{array}{l}18.4 \\
22.6\end{array}$ & $\begin{array}{l}9.0 \\
9.4\end{array}$ & $\begin{array}{l}22.1 \\
20.5\end{array}$ & $\begin{array}{l}19.3 \\
22.1\end{array}$ & $\begin{array}{l}18.7 \\
19.9\end{array}$ \\
\hline & 5.51 & 8.1 & 11.3 & 21.8 & 20.4 & 10.1 & 19.8 & 19.6 & 20.8 \\
\hline & 5.34 & 10.9 & 12.2 & 22.0 & 24.0 & 10.7 & 19.1 & $22 \cdot 3$ & 21.8 \\
\hline
\end{tabular}

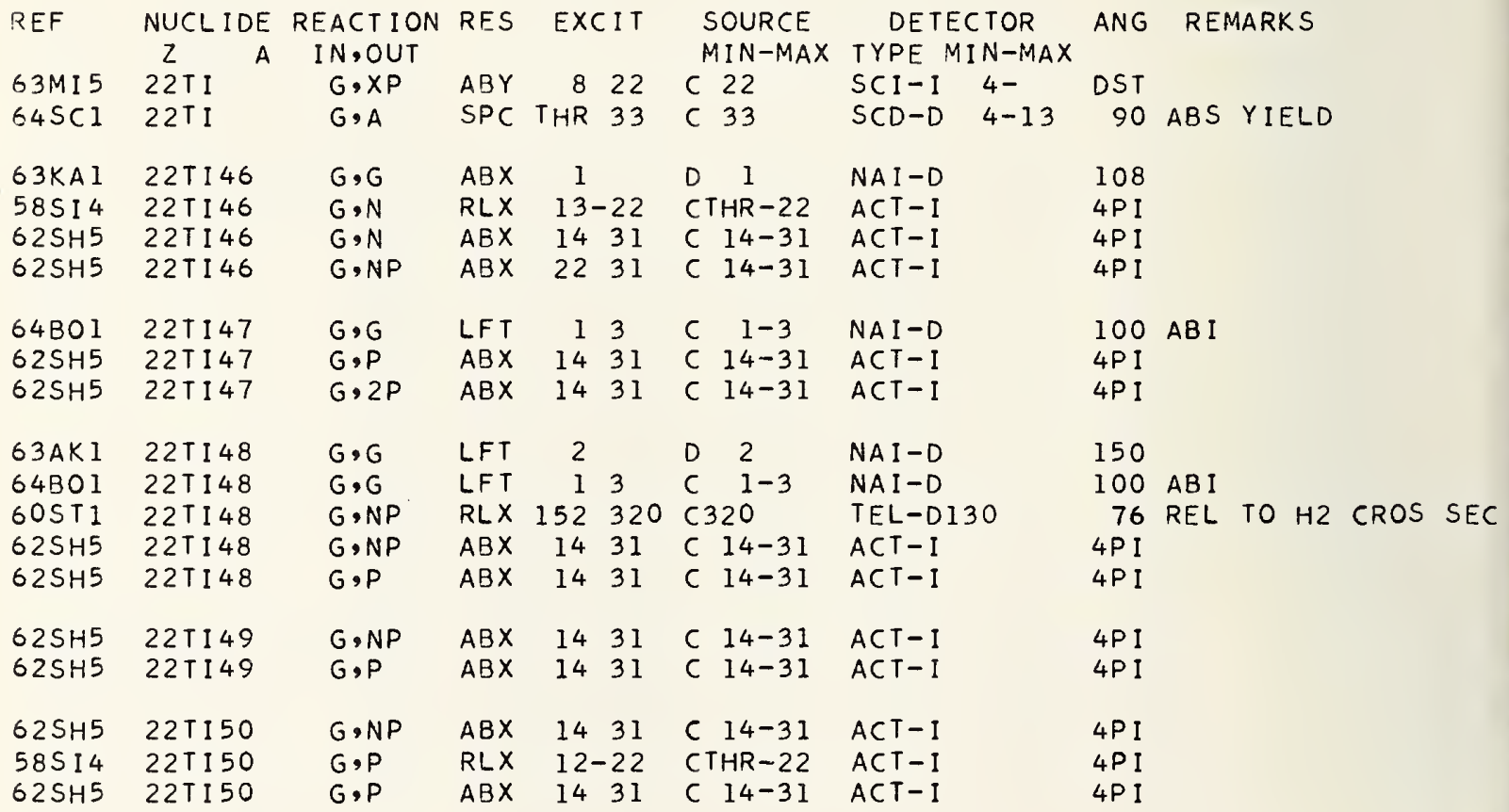




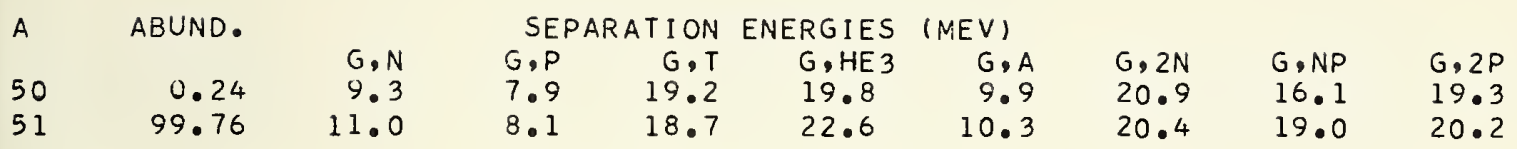

\begin{tabular}{|c|c|c|c|c|c|c|c|c|c|c|c|}
\hline REF & NUCL & I DE & REACTION & RES & $E \times C$ & IT & SOURCE & DET & CTOR & ANG & REMARKS \\
\hline & $z$ & A & IN,OUT & & & & MIN-MAX & TYPE & IN-MAX & & \\
\hline 6 JRE I & $23 \mathrm{~V}$ & & $G, G$ & $A B X$ & 7 & & $D$ & NAI I D & & 90 & \\
\hline $5 \in H E 2$ & $23 v$ & & $G, N$ & RLY & THR - & -31 & C 31 & & & & * REL TO ALPHAS \\
\hline $61 B A 2$ & $23 V$ & & $G, \times N$ & $A B Y$ & THR & 22 & C 22 & THR-I & $5-$ & DST & \\
\hline $58 \mathrm{HAl}$ & $23 \mathrm{~V}$ & & $G, P$ & SPC & THR - & -30 & C 30 & $E M U-D$ & & DST & * \\
\hline $63 M I 5$ & $23 \mathrm{~V}$ & & $G, \times P$ & $A B Y$ & 5 & 22 & C 22 & $S C I-I$ & 4- & DST & \\
\hline $56 \mathrm{HE} 2$ & $23 \mathrm{~V}$ & & $G, A$ & RLY & THR - & -31 & C 31 & & & & * REL TO NEUTRONS \\
\hline $58 \mathrm{TO2}$ & $23 \mathrm{~V}$ & & $G, A$ & $A B Y$ & 8 & 22 & C 22 & EMU-I & & DST & \\
\hline $63 K R 1$ & $23 \mathrm{~V}$ & & $G, A$ & RLY & 8 & 30 & C 21,30 & $S C D-1$ & & 90 & \\
\hline $62 K E 1$ & $23 V$ & 51 & $E, E /$ & $S P C$ & 0 & 17 & D183-600 & TEL-D & & DST & SOURCE $183,300,600$ \\
\hline $58 \mathrm{CH} 2$ & $23 \mathrm{~V}$ & 51 & $G, N$ & RLY & THR & & CTHR & $B F 3-1$ & & $4 \mathrm{PI}$ & THRESHOLD \\
\hline $60 \mathrm{GE} 3$ & $23 \mathrm{~V}$ & 51 & $G, N$ & NOX & THR & & CTHR & $B F 3-1$ & & $4 \mathrm{PI}$ & THRESHOLD \\
\hline $62 \mathrm{FUI}$ & $23 \mathrm{~V}$ & 51 & $G, N$ & $A B X$ & 11 & 28 & D $8-28$ & $B F 3-I$ & & $4 \mathrm{PI}$ & QUADRUPOLE MOMENT \\
\hline $62 \mathrm{FUI}$ & $23 \mathrm{~V}$ & 51 & $G \cdot 2 N$ & $A B X$ & 20 & 28 & $8-28$ & $B F 3-I$ & & $4 \mathrm{PI}$ & QUADRUPOLE MOMENT \\
\hline $58 \mathrm{KAl}$ & $23 v$ & 51 & $G, \times N$ & $A B X$ & 12 & 22 & C $12-22$ & $B F 3-1$ & & $4 P I$ & \\
\hline $62 \mathrm{FUI}$ & $23 \mathrm{~V}$ & 51 & $G, N P$ & $A B X$ & 20 & 28 & D $8-28$ & $B F 3-I$ & & $4 \mathrm{PI}$ & QUADRUPOLE MOMENT \\
\hline $57 E R I$ & $23 V$ & 51 & $G, A$ & $A B I$ & 8 & 32 & C 32 & $A C T-I$ & & 4PI & \\
\hline 59DY 1 & $23 \mathrm{~V}$ & 51 & $G, A$ & $A B X$ & 14 & 25 & C 25 & $A C T-I$ & & 4PI & \\
\hline $61 C A 2$ & $23 \mathrm{~V}$ & 51 & $G, A$ & $A B X$ & THR & 32 & CTHR-32 & $A C T-I$ & & $4 P I$ & \\
\hline $62 K R 1$ & $23 \mathrm{~V}$ & 51 & $G, A$ & AB I & 10 & 30 & C 21,30 & $S C D-D$ & & 90 & \\
\hline
\end{tabular}

CHROMIUM $Z=24$

\begin{tabular}{|c|c|c|c|c|c|c|c|c|c|}
\hline A & ABUND. & & SEF & ATION & ENERG IES & (MEV) & & & \\
\hline 50 & 4.31 & $\begin{array}{r}\mathrm{G}, \mathrm{N} \\
12.9\end{array}$ & $\begin{array}{l}G, P \\
9.6\end{array}$ & $\begin{array}{r}G . T \\
23.2\end{array}$ & $\begin{array}{l}\text { G. HE3 } \\
20.3\end{array}$ & $\begin{array}{l}G . A \\
8.6\end{array}$ & $\begin{array}{l}\mathrm{G}, 2 \mathrm{~N} \\
23.3\end{array}$ & $\begin{array}{l}G, N P \\
21.2\end{array}$ & $\begin{array}{l}G, 2 P \\
16.3\end{array}$ \\
\hline 52 & 83.76 & 12.0 & 10.5 & 22.4 & 21.8 & 9.4 & 21.3 & 21.6 & $18 \cdot 6$ \\
\hline $\begin{array}{l}53 \\
54\end{array}$ & $\begin{array}{l}9.55 \\
2.38\end{array}$ & $\begin{array}{l}7.9 \\
9.7\end{array}$ & $\begin{array}{l}11.1 \\
12 .\end{array}$ & $\begin{array}{l}21.0 \\
19.7\end{array}$ & $\begin{array}{l}18.8 \\
22.1\end{array}$ & $\begin{array}{l}9.1 \\
7.9\end{array}$ & $\begin{array}{l}20.0 \\
17.6\end{array}$ & $\begin{array}{l}18.5 \\
20.9\end{array}$ & $\begin{array}{l}20.1 \\
22 .\end{array}$ \\
\hline
\end{tabular}

REF

NUCLIDE REACTION RES EXCIT

SOURCE DETECTOR ANG REMARKS

$Z$ A IN,OUT

6ORE 1 24CR

57FE2 24CR

$58 C 01 \quad 24 C R$

61BA2 24CR

$64 \mathrm{CO} 2 \quad 24 \mathrm{CR}$

$\begin{array}{llrr}G, G & A B X & 7 & \\ G, N & A B Y & 15 & 30 \\ G, N & S P C & 6 & 30 \\ G, X N & A B Y & T H R & 22 \\ G, X N & A B Y & T H R-80\end{array}$

MIN-MAX TYPE MIN-MAX

6

64BO1 24CR52

64BE3 24CR52

6OGE3 24CR52

$G, N \quad A B X \quad 20$

D 7 NAI $-D$

C 14-30 THR-I

C 20,30

90

C 22

THR-1

$2-14$

90 THRESHOLD

64BO1 24CR53

6OGE3 24CR53

$G, G \quad$ LFT 13

C 80

BF 3-I

DST

4P I

E,El FMF 09 DI50,180 MAG-D

G,N NOX THR CTHR BF3-I

4PI

$100 \mathrm{ABI}$

DST J-PI

4PI THRESHOLD

$G, G \quad L F T \quad 1 \quad 3 \quad C \quad 1-3$ NAI-D 100 ABI

$G, N$ NOX THR CTHR BF3-I

4 PI THRESHOLD 


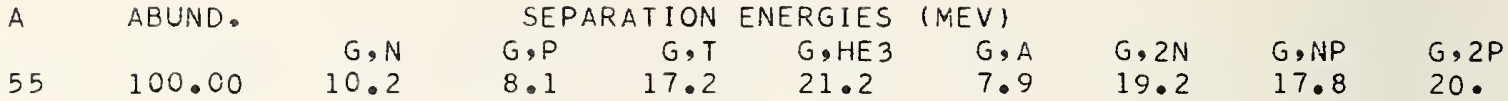

\begin{tabular}{|c|c|c|c|c|c|c|c|c|c|c|c|c|}
\hline REF & NUCL IDE & REACTION & RES & $E \times C$ & & & OURCE & DET & CTOR & ANG & REMARKS & \\
\hline & A & IN,OUT & & & & & IN-MAX & TYPE N & IN-MAX & & & \\
\hline $\begin{array}{l}56 F U 1 \\
60 R E 1\end{array}$ & $\begin{array}{l}25 M N 55 \\
25 M N 55\end{array}$ & $\begin{array}{l}G, G \\
G, G\end{array}$ & $\begin{array}{l}A B X \\
A B X\end{array}$ & $\begin{array}{l}44 \\
7\end{array}$ & 40 & $\begin{array}{l}C \\
D\end{array}$ & $\begin{array}{l}4-40 \\
7\end{array}$ & $\begin{array}{l}\text { NAI I D } \\
\text { NAI I D }\end{array}$ & & $\begin{array}{r}120 \\
90\end{array}$ & & \\
\hline $64 \mathrm{BO}$ & $25 M N 55$ & $G, G$ & LFT & 1 & 3 & $c$ & $1-3$ & NAI -D & & 100 & $A B I$ & \\
\hline $58 \mathrm{CH} 2$ & $25 M N 55$ & $G, N$ & RLY & THR & & $C T$ & $H R$ & $B F 3-I$ & & 4PI & THRESHOLD & \\
\hline 59PA2 & $25 M N 55$ & $G, N$ & $A B X$ & 10 & 24 & $c$ & $10-24$ & BF 3-I & & 4P I & & \\
\hline 60GE 3 & $25 M N 55$ & $G, N$ & NOX & THR & & $C T$ & HR & $B F 3-I$ & & $4 P I$ & THRESHOLD & \\
\hline GITA1 & $25 M N 55$ & $G, N$ & $\operatorname{NOX}$ & 162 & 22 & $c$ & 22 & $T H R-I$ & $6-$ & 90 & & \\
\hline $57 \mathrm{HI} 1$ & $25 M N 55$ & $E, 3 N$ & $A B X$ & $30 \epsilon$ & 60 & D & 64,82 & $A C T-I$ & & 4PI & & \\
\hline $58 \mathrm{KAl}$ & $25 M N 55$ & $G, \times N$ & $A B X$ & 112 & 22 & $c$ & $11-22$ & $B F 3-I$ & & $4 P I$ & & \\
\hline $60 F L 1$ & $25 M N 55$ & $G, \times N$ & $A B X$ & 11 & 27 & $c$ & $12-30$ & BF 3-I & & 4PI & QUADRUPOLE & MOMENT \\
\hline $616 A 2$ & $25 M i N 55$ & $G, \times N$ & $A B Y$ & THR & 22 & $c$ & 22 & THR-I & $5-$ & DST & & \\
\hline $64 \mathrm{CO} 2$ & $25 M N 55$ & $G, \times N$ & $A B Y$ & $T H R-\varepsilon$ & & $c$ & 80 & BF 3-I & & 4P I & & \\
\hline$S I C A 1$ & 25 MN 56 & $D, G$ & $A B X$ & 16 & 18 & $D$ & $3-5$ & $A C T-I$ & & $4 P I$ & & \\
\hline
\end{tabular}

A ABUND.

$\begin{array}{rr}54 & 5.82 \\ 56 & 91.66 \\ 57 & 2.19 \\ 58 & 0.33\end{array}$

REF

$\begin{array}{ll} & Z \\ 59 K O 1 & 26 \mathrm{FE} \\ 60 R E 1 & 26 \mathrm{FE} \\ 63 \mathrm{SU1} & 26 \mathrm{FE} \\ 64 \mathrm{BO} 1 & 26 \mathrm{FE} \\ 57 \mathrm{HE} 1 & 26 \mathrm{FE} \\ 55 \mathrm{BA} 5 & 26 \mathrm{FE} \\ 55 \mathrm{DI} 1 & 26 \mathrm{FE} \\ 56 \mathrm{HA1} & 26 \mathrm{FE} \\ 61 \mathrm{BA2} & 26 \mathrm{FE} \\ 64 \mathrm{CO} 2 & 26 \mathrm{FE} \\ 57 \mathrm{HE} 1 & 26 \mathrm{FE} \\ 63 \mathrm{MI} 5 & 26 \mathrm{FE} \\ 58 \mathrm{TO} 2 & 26 \mathrm{FE} \\ 63 \mathrm{KR} 1 & 26 \mathrm{FE}\end{array}$

55DE1 26FE53

55DE 1 26FE 54 57CA2 26FE54 62DE 1 26FE54 $58 G 03$ 26FE 54 58603 26FE54

\begin{tabular}{rrrr} 
& \multicolumn{2}{c}{ SEPARATION } & ENERGIES \\
G,N & G,P & G,T & G, HE 3 \\
13.6 & 8.9 & 22.9 & 19.7 \\
11.2 & 10.2 & 20.9 & 20.3 \\
7.6 & 10.6 & 19.6 & 18.2 \\
10.0 & 12.0 & 19.4 & 22.0
\end{tabular}

(MEV)
G.A
8.4
7.6
7.3
7.6

$\begin{array}{lll}G, 2 N & \text { G,NP } & \text { G,2P } \\ 24.1 & 20.9 & 15.4 \\ 20.5 & 20.4 & 18.3 \\ 18.8 & 17.9 & 19.6 \\ 17.7 & 20.6 & 21.4\end{array}$

IRON $\quad Z=26$ 
$61 K E 2 \quad 26 F E 56$

$58 T 01$ 26FE56

SOGE3 26FE 56

$58 T 01$ 26FE 57

6OGE 3 26FE 57
$G, G$

$G, N$

NOX

$\begin{array}{lrr}\text { RLY } & 12 & 22\end{array}$

D 1

NOX THR CTHR

NAI - D

BF 3-I

BF 3-I

BF 3-I

BF 3-I
ANG REMARKS

G-WDTH

4PI THRESHOLD

4PI THRESHOLD

4PI THRESHOLD

4PI THRESHOLD

\section{COBALT $Z=27$}

\begin{tabular}{|c|c|c|c|c|c|c|c|c|c|}
\hline A & ABUND. & & SEF & ATION & ENERG IES & (MEV) & & & \\
\hline & & $G, N$ & $G, P$ & $G, T$ & G, HE 3 & $G, A$ & $G, 2 N$ & $G, N P$ & $G, 2 P$ \\
\hline 59 & 100.00 & 10.5 & $7 \cdot 4$ & 16.6 & $20 \cdot 3$ & 7.0 & 19.0 & $17 \cdot 4$ & $19 \cdot 3$ \\
\hline
\end{tabular}

REF NUCLIDE REACTION RES EXCIT SOURCE DETECTOR ANG REMARKS

6OREI 270059 A IN,OUT MIN-MAX TYPE MIN-MAX

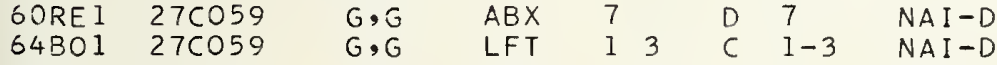

61CRI 27CO59 E,E NOX O 4 DI83 MAG-I

$58 \mathrm{CH2} 27 \mathrm{CO} 59$ G,N RLY THR CTHR BF3-I

6OGE3 27CO59 G,N NOX THR CTHR BF3-I

62CAI 27CO59 G,N NOX 1130 C 30 ACT-I

62 FU1 270059

$62 F \cup 1270059$

$G, N \quad A B X \quad 1128$

$G, 2 N \quad A S X \quad 1928$

C $9-28$ BF $3-I$

$56 \mathrm{HAl} 27 \mathrm{CO} 59$

$G, X N$

ABX 15,18

C 9-28

$B F 3-I$

$56 \mathrm{HE} 1 \quad 27 \mathrm{CO} 59$

58KA1 27C059

$G, X N$

$G, X N$

RLY IC-31

D 15,18

BF 3-I

5SWAI 27C059

SOFLI 27C059

6IBA2 27CO59

SIEMI 27CO59

64BA2 27CO59

$64 \mathrm{CO} 227 \mathrm{CO} 59$

62 FU1 270059

56W01 27C059

60GO2 27CO59

56WO1 27C059

56WOI 27CO59

56́WO $127 C 059$

56WO1 27C059

56WO1 27CO59

56W01 27C059

56WO1 27CO59

$56 \mathrm{FO} 127 \mathrm{CO} 59$

$6 I F O 1 \quad 27 C 059$

$60 \mathrm{CHI} 27 \mathrm{CO} 5 \%$

$63 M I 5 \quad 27 C 059$

$60 G 02 \quad 270059$

$56 \mathrm{FOI} 27 \mathrm{CO} 59$

6IFO1 27C059

$60 \mathrm{CH} 127 \mathrm{CO} 59$

$56 \mathrm{HE} 127 \mathrm{CO} 59$

56WAI 27CO59

$60 \mathrm{CH} 1 \quad 27 \mathrm{CO} 59$

$60 \mathrm{GO} 2 \quad 27 \mathrm{CO} 59$

$G, X N$

$G, X N$

$G, X N$

$G, X N$

$G, \times N$

$G, X N$

ABX 1022

C 31

RLY 10-31

c 10-22

BF 3-I

ABX II 24

c 31

ACT - I

$A B Y$ THR 22

C 12-30

SPC 1330

C 22

BF 3-I

c 30

THR - I

$A B X \quad 10-30$

c 10-30

$G, N P$

$A B Y$ THR -80

C 80

EMU-D

BF 3-I

$G, N 2 P$

$A B X \quad 18 \quad 28$

c $9-28$

BF 3-I

$G, N 2 P \quad A B X \quad 30260$

ABY THR -309 C 309

$B F 3-I$

C $30-260$

$A C T-I$

G, $5 N P$ ABY THR-309 C309

$A C T-I$

$G, 5 N 2 P$

ABY THR-309 C309

$A C T-I$

G.6NP ABY THR-309 C309

$A C T-I$

G, 6N2P ABY THR-309 C309

$A C T-I$

G, 7N3P ABY THR-309 C 309

$A C T$ - I

G.8N4P ABY THR-309 C 309

$A C T-I$

$A C T-I$

G,9N5P ABY THR-309 C309

$G, P$

RLY

730

c 30

$G, P$

$G, X P$

SPC $\quad 7 \quad 30 \quad C \quad 30$

$G, X P$

$\begin{array}{lllll}\text { RLX } & 22 & 90 & C & 90\end{array}$

$A C T-I$

EMU-D

EMU-D

TEL - I

ANG

90

$100 \mathrm{ABI}$

DST G-WDTH, MULTIPOLES

4PI THRESHOLD

4PI THRESHOLD

4PI ISOMER RATIO

$4 P$ I

$4 P I$

$4 P I$

4P I

* REL TO TRITONS

4PI * REL TO TRITONS

4PI QUADRUPOLE MOMENT

G,PD ABX 30260 C $30-260$ ACT-I

$G . D \quad R L Y \quad 1530 \quad C \quad 30$ EMU-D

G,D RLY 1530 C 30 EMU-D

G,XD RLX $30 \quad 90 \quad C \quad 90$

EMU-D

$G, T$

RLY $16-31$

c 31

G,T RLY 16-3I C 31

ACT - I

G,XT RLY 3290 C 90

TEL-I I5-30

$G, H E 3 \quad A B X$

30260 C 30-260 ACT-I

722 C 22 EMU-I

$G, A \quad A B Y$

930

c 30

EMU-D

DST

2-12 90

4P I

$4 P I$

4P I

$4 P I *$

4PI ALSO G,PD, G,HE3

$4 P I *$

4PI *

4PI *

4PI *

$4 P I *$

$4 P I *$

$4 \mathrm{PI} *$

90 YLD REL TO G,D

DST

$61 \mathrm{FO} \quad 27 \mathrm{CO} 59$

$G, A \quad S P C$

DST

4PI ALSO G, N2P, G,HE 3

90 YLD REL TO G,P

DST NO DEUTERONS FOUND

90 REL TO PROTONS

* REl to nEUtrons $4 P I *$ REL TO NEUTRONS

90 REL TO DEUTERONS $4 P I A L S O G, N 2 P, G, P D$

DST

DST
$\mathrm{Fe}$
$\mathrm{Co}$ 


\begin{tabular}{|c|c|c|c|c|c|c|c|c|c|}
\hline A & ABUND. & & SE & T I ON & ENERGIES & (MEV) & & & \\
\hline 58 & 67.88 & $\begin{array}{r}G, N \\
12.2\end{array}$ & $\begin{array}{l}G, P \\
8.2\end{array}$ & $\begin{array}{r}G, T \\
21.1\end{array}$ & $\begin{array}{l}\text { G, HE } 3 \\
17.7\end{array}$ & $\begin{array}{l}G . A \\
6.4\end{array}$ & $\begin{array}{l}G, 2 N \\
22.5\end{array}$ & $\begin{array}{l}\text { G,NP } \\
19.6\end{array}$ & $\begin{array}{l}G, 2 P \\
14.2\end{array}$ \\
\hline $\begin{array}{l}60 \\
61 \\
62 \\
64\end{array}$ & $\begin{array}{r}26.23 \\
1.19 \\
3.66 \\
1.08\end{array}$ & $\begin{array}{r}11.4 \\
7.8 \\
10.6 \\
9.7\end{array}$ & $\begin{array}{r}9.5 \\
9.9 \\
11.1 \\
12.5\end{array}$ & $\begin{array}{l}20.1 \\
19.3 \\
19.5 \\
19.1\end{array}$ & $\begin{array}{l}19.2 \\
17.0 \\
21.0 \\
22.9\end{array}$ & $\begin{array}{l}6.3 \\
6.5 \\
7.0 \\
8.0\end{array}$ & $\begin{array}{l}20.4 \\
19.2 \\
18.4 \\
16.5\end{array}$ & $\begin{array}{l}20.0 \\
17.4 \\
20.5 \\
21.0\end{array}$ & $\begin{array}{l}16.9 \\
18.1 \\
19.8\end{array}$ \\
\hline
\end{tabular}

\begin{tabular}{|c|c|c|c|c|c|c|c|c|c|c|c|}
\hline REF & $\underset{Z}{\text { NUCL IDE }}$ & $\begin{array}{l}\text { REACT ION } \\
\text { IN, OUT }\end{array}$ & RES & $E X$ & I T & & $\begin{array}{l}\text { OURCE } \\
\text { I IN-MAX }\end{array}$ & $\begin{array}{l}\text { DETE } \\
\text { TYPE }\end{array}$ & $\begin{array}{l}\text { ECTOR } \\
\text { IIN-MAX }\end{array}$ & ANG & REMARKS \\
\hline 61MII & $28 N I$ & $G, M U-T$ & $A B X$ & 6 & 22 & D & $6-22$ & NAI I D & & & \\
\hline $56 \mathrm{FU1}$ & $28 N I$ & $G, G$ & $A B X$ & 4 & 40 & $c$ & $4-40$ & NAI -D & & 120 & \\
\hline $60 \mathrm{KAl}$ & $28 N I$ & $G, G$ & LFT & 1 & & D & 1 & & & & * $A B X$ \\
\hline 6ORE I & $28 \mathrm{NI}$ & $G, G$ & $A B X$ & 7 & & D & 7 & NAI -D & & 90 & \\
\hline 61TO1 & $28 \mathrm{NI}$ & $G, G$ & $A B X$ & 4 & 14 & $c$ & $4-14$ & NAI -D & & 120 & \\
\hline $62 \mathrm{BE} 2$ & $28 \mathrm{NI}$ & $G, G$ & $A B X$ & 5 & 9 & D & $5-9$ & $N A I-D$ & & 135 & \\
\hline 64AR 1 & $28 N I$ & $G, G$ & $A B X$ & 8 & & D & 8 & NAI -D & & DST & $J$, WIDTH \\
\hline $64 \mathrm{GII}$ & $28 N I$ & $G, G$ & NOX & 8 & & D & 8 & $N A I-D$ & & 135 & WIDTH \\
\hline $56 \mathrm{HA} 1$ & $28 \mathrm{NI}$ & $G, X N$ & $A B X$ & 15 & 18 & D & 15,18 & BF 3-I & & 4PI & \\
\hline $61 B A 2$ & $28 \mathrm{NI}$ & $G, \times N$ & $A B Y$ & THR & 22 & $c$ & 22 & THR - I & $5-$ & DST & \\
\hline $64 \mathrm{CO} 2$ & $28 N I$ & $G, \times N$ & $A B Y$ & THR & 80 & $c$ & 80 & BF 3-I & & 4PI & \\
\hline 55DL1 & $28 \mathrm{NI}$ & $G, P$ & SPC & 18 & & D & 18 & EMU-D & & & \\
\hline 55 & $28 \mathrm{NI}$ & $G, P$ & RLY & THR & 65 & C & 65 & $S \subset I-D$ & $14-$ & DST & \\
\hline 56LE 1 & $28 \mathrm{NI}$ & $G, P$ & $A B X$ & 8 & 28 & $c$ & $21-28$ & EMU-I & & DST & \\
\hline $57 R 01$ & $28 \mathrm{NI}$ & $G, P$ & $R L x$ & 15 & 18 & D & 15,18 & & & & * REL TO CU63 $(\mathrm{G}, \mathrm{N})$ \\
\hline $575 P 1$ & $28 \mathrm{NI}$ & $G, P$ & SPC & 9 & 18 & c & 18 & $E M U-D$ & $1-9$ & DST & \\
\hline 62 SE2 & $28 N I$ & $G, P$ & $A B X$ & 15 & 18 & D & 15,18 & $S C I-I$ & & 0 & \\
\hline $57 B A 2$ & $28 \mathrm{NI}$ & $G, X P$ & SPC & & & $c$ & 85 & TEL-D & $20-65$ & & * \\
\hline $58 B A 6$ & $28 \mathrm{NI}$ & $G, X P$ & & & & $c$ & 85 & & $15-65$ & & * QUAS I-DEUTERON \\
\hline $60 \mathrm{CH} 1$ & $28 \mathrm{NI}$ & $G, \times P$ & $R L X$ & THR & 90 & $c$ & 90 & TEL-I & $15-30$ & 90 & REL TO DEUTERONS \\
\hline 61MA2 & $28 N I$ & $G, X P$ & NOX & 8 & 21 & $c$ & 18,21 & $S C I-D$ & $1-10$ & DST & \\
\hline 63415 & $28 N I$ & $G, \times P$ & $A B Y$ & 8 & 22 & $c$ & 22 & $S C I-I$ & 4- & DST & \\
\hline 63YA2 & $28 \mathrm{NI}$ & $G, D$ & RLY & 15 & 26 & $c$ & 26 & $E M U-D$ & $3-9$ & 90 & REL TO PROTONS \\
\hline $60 \mathrm{CHI}$ & $28 \mathrm{NI}$ & $G, X D$ & $R L X$ & THR & 90 & $c$ & 90 & TEL-I & $15-30$ & 90 & REL TO PROTONS \\
\hline $60 \mathrm{CHI}$ & $28 \mathrm{NI}$ & $G, \times T$ & RLY & THR & 90 & c. & 90 & TEL-I & $15-30$ & 90 & REL TO DEUTERONS \\
\hline $57 \mathrm{BO} 1$ & $28 \mathrm{NI}$ & $G, A$ & $A B X$ & THR - & 31 & $c$ & 31 & $E M U-D$ & & DST & * SPC \\
\hline $58 \mathrm{TO} 2$ & $28 N I$ & $G, A$ & $A B Y$ & 6 & 22 & $c$ & 22 & EMU-I & & DST & \\
\hline $63 K R 1$ & $28 \mathrm{NI}$ & $G, A$ & $S P C$ & 6 & 30 & $c$ & 21,30 & $S C D-D$ & $1-13$ & 90 & RELATIVE YIELDS \\
\hline $64 S C 1$ & $28 \mathrm{NI}$ & $G, A$ & $S P C$ & THR & 33 & C & 33 & $S C D-D$ & $4-13$ & 90 & $A B S$ YIELD \\
\hline $64 B 01$ & $28 N I 58$ & $G, G$ & LFT & 1 & 3 & $c$ & $1-3$ & NAI - D & & 100 & $A B I$ \\
\hline $61 C R 1$ & $28 N I 58$ & $E, E /$ & NOX & 0 & 8 & D. & & $M A G-D$ & & DST & G-WDTH, MULTIPOLES \\
\hline $59 C A 4$ & 28 NI 58 & $G, N$ & $A B X$ & 12 & 32 & c & $12-32$ & $A C T-I$ & & 4PI & \\
\hline $59 R O 2$ & $28 N I 58$ & $G, N$ & $A B X$ & 12 & 24 & c & 24 & $A C T-I$ & & 4PI & \\
\hline $59 C A 4$ & $28 N I 58$ & $G, N P$ & $A B X$ & 12 & 32 & c & $12-32$ & $A C T-I$ & & 4P I & \\
\hline $59 C A 4$ & 28 NI 58 & $G, P$ & $A B X$ & 12 & 32 & C & $12-32$ & $A C T-I$ & & 4PI & \\
\hline 56ME2 & $28 N 160$ & $G, G$ & LFT & 1 & & & 1 & NAI -D & & DST & $J-P I$ \\
\hline 610 & $28 N 160$ & $E, E /$ & NOX & 0 & 5 & & 83 & MAG-D & & DST & G-WDTH, MULTIPOLES \\
\hline $59 C A 4$ & $28 N I 62$ & $G, P$ & $A B X$ & 12 & 32 & c & $12-32$ & $A C T-1$ & & 4PI & \\
\hline
\end{tabular}




\begin{tabular}{|c|c|c|c|c|c|c|c|c|c|}
\hline A & ABUND. & & $S E$ & ATION & ENERGIES & (MEV) & & & \\
\hline 63 & 69.09 & $\begin{array}{r}G, N \\
10.8\end{array}$ & $\begin{array}{l}G, P \\
6.1\end{array}$ & $\begin{array}{r}G, T \\
16.1\end{array}$ & $\begin{array}{l}\text { G, HE } 3 \\
18.9\end{array}$ & $\begin{array}{l}G, A \\
5.8\end{array}$ & $\begin{array}{l}G, 2 \mathrm{~N} \\
19.7\end{array}$ & $\begin{array}{l}G, N P \\
16.8\end{array}$ & $\begin{array}{l}G, 2 P \\
17.2\end{array}$ \\
\hline 65 & 30.91 & 9.9 & 7.4 & 15.5 & 20.7 & 6.8 & 17.8 & 17.1 & 19.9 \\
\hline
\end{tabular}

REF NUCLIDE REACTION RES EXCIT SOURCE DETECTOR ANG REMARKS

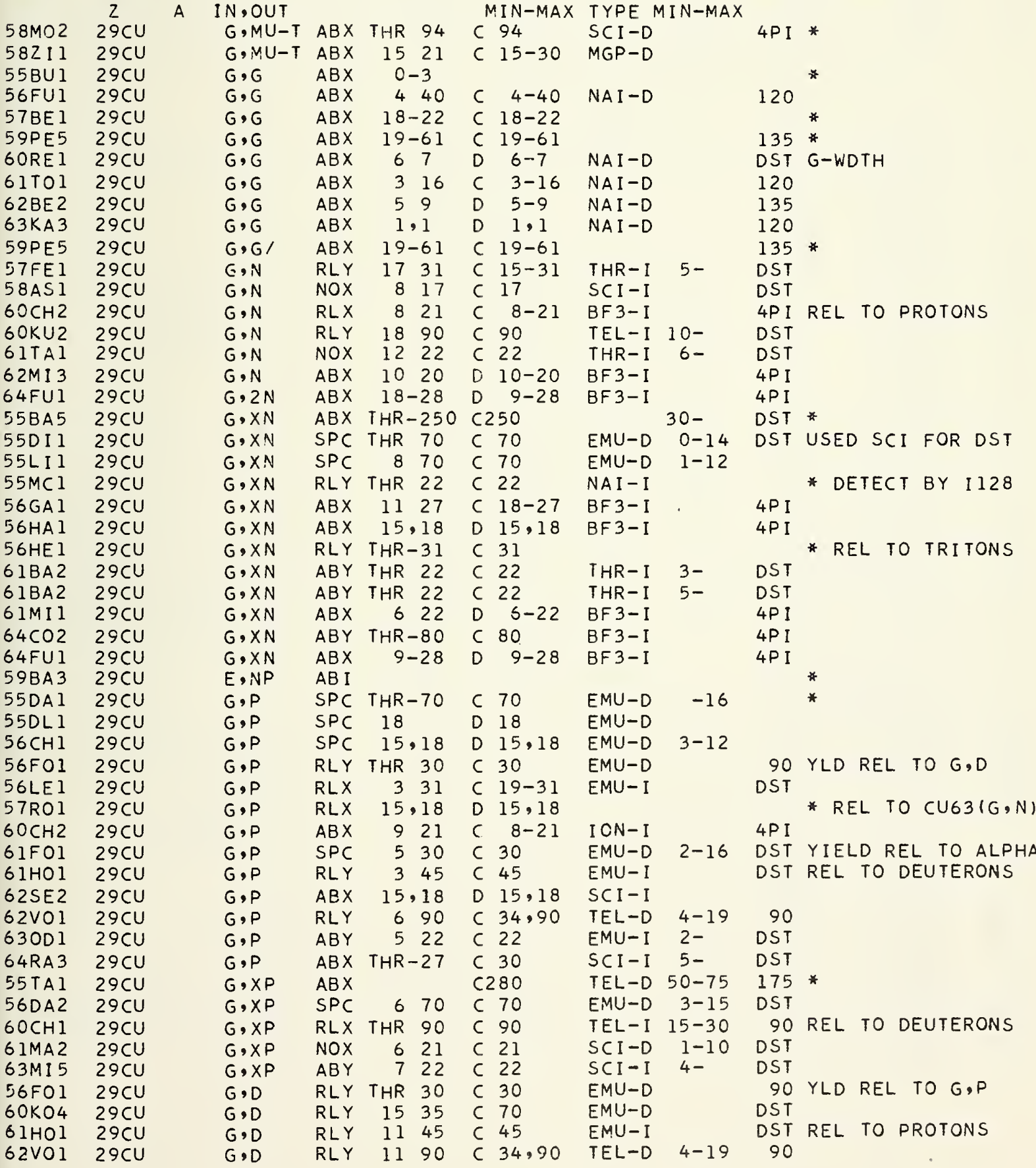


REF NUCLIDE REACTION RES EXCIT $Z$ A IN,OUT

63YA2 29CU G,D

$64 \mathrm{SH} 329 \mathrm{CU}$

$60 \mathrm{CHI} 29 \mathrm{CU}$

$56 \mathrm{HEI} 29 \mathrm{CU}$

$62 \mathrm{VO} 229 \mathrm{CU}$

$60 \mathrm{CH} 229 \mathrm{CU}$

$57 B 0129 \mathrm{CU}$

$58 \mathrm{TO} 2 \quad 29 \mathrm{CU}$

$61 \mathrm{FO} 2 \mathrm{CU}$

63 KR $1 \quad 29 \mathrm{CU}$

S4SCl $29 \mathrm{CU}$

61CA1 29CU60

$60 B 0329$ CU 63

$61 R O 129$ CU 63

$62 B 06 \quad 29$ CU63

$63 M C 1 \quad 29 C 463$

$64 \mathrm{BO} 2 \mathrm{CU} 63$

55 CAl 29CU63

$55 S C 1 \quad 29 C U 63$

58BE 1 29CU63

59NA1 29CU63

59PE3 29CU63

60GE3 29CU63

61CO2 29CU63

62DE 1 29CU63

$64 \mathrm{FU} 129 \mathrm{CU}_{6} 3$

56WAI 29CU63

64 FU1 29CU63

57 HII 29 CU 63

OOYAI 29 CU63

$62 \mathrm{CH} 2 \quad 29 \mathrm{CU} 63$

$63 \mathrm{GO} 829 \mathrm{CU}^{3} 3$

$62 \mathrm{CH} 2 \quad 29 \mathrm{CU}_{6} 3$

56WA1 29CU63

$58 T 02 \quad 29$ CU 63

$63608 \quad 29$ CU 63

6OSTI 29CU64

$60 B 03 \quad 29 C U 65$

$63 \mathrm{KA} 129 \mathrm{CU} 65$

64BE6 29CU65

64BOI 29CU65

$58 B E 1$ 29CU65

$60 G E 3$ 29CU65

$61 \mathrm{CO} 2$ 29CU65

64FU1 29CU65

6OAII 29CU65

56WA1 29CU65

64FU1 29CU65

5605129 CU65

6OLII 29CU65

$62 \mathrm{CH} 229 \mathrm{CU} 65$

$62 \mathrm{CH} 2 \quad 29 \mathrm{CU} 65$

56WA1 29CU65

57 ERI 29CU65
RLY 1426

G, D RLY 15-40

$G, X D$ RLX THR 90

$G, T$ RLY THR-3I

G,T RLY 1590

$G, X T$ RLY THR 90

$G, A \quad A B X$ THR-3I

$G, A$

$G, A$

$G, A$

$G, A$

$D, G$

$G, G$

$G, G$

$G, G$

$G, G$

$G, G$

$G, N$

$G, N$

$G, N$

$G, N$

$G, N$

$G, N$

$G, N$

$G, N$

$G, 2 N$

$G, \times N$

$G, \times N$

$E, N$

$G, P$

$G, X P$

$G, D$

$G, \times D$

$G, T$

$G, A$

$G, A$

$G, N P$

$G, G$

$G, G$

$G, G$

$G, G$

$G, N$

$G, N$

$G, N$

$G, 2 N$

$G, 3 N$

$G, X N$

$G, X N$

$G, P$

$G, P$

$G, X P$

$G, X D$

$G, T$

$G, A$

SPC 722

$\begin{array}{lll}\text { RLY } & 7 & 22\end{array}$

SPC 930

SPC THR 33

$A B X \quad 1416$

LFT 02

$A B X \quad 0 \quad 1$

LFT

1

LFT 13

RLY 18

ABX 1020

RLY 1011

ABX 18

$A B X \quad 1218$

$A B X 21$

$A B X \quad 19-28$

RLY $11-31$

$A B X \quad 10-28$

$A B \times \quad 10 \quad 82$

$A B X \quad 15,18$

RLY 690

142

RLX 15,18

$A B X \quad 16-28$

$A B I$

$29 \quad 110$

RLY 10-31

$A B X \quad 10-28$

$S P C$

$A B X$

1228

RLY 790

RLY 15-31
SOURCE
MIN-MAX TYY TECTOR
MIN-MAX

C 26 EMUTD 3-9

C $24-40$

C 90

C 31

C 34,90

C 90

C 31

C 22

C 30

C 21,30

C 33

C $3-5$

C $0-2$ NAI-D

D $0-1$ NAI-D

LFT $\quad 0 \quad 4 \quad C \quad 0-4 \quad$ NAI $-D$

D 1

$N A I-D$

C 1 -3

NAI $-D$

$A C T-I$

$A C T-I$

- 18

C 12-20

C 10-11

D 18

$A C T-I$

$B F 3-I$

RLY 1011 C $10-11$ ACT-I

NOX THR CTHR

$B F 3-I$

D 21

$A C T$ - I

$A C T-I$

D $10-28$

BF 3-I

C 31

ACT - I

D $10-28$

D $30-82$

BF 3-I

$A C T-I$

D 15,18

$A C T-I$

C 34-90

$T E L-D$

EMU-I

C 22

$T E L-D$

$A C T-I$

EMU-I

EMU-I

TEL-D 130

$\mathrm{RLX} 144 \quad 320 \quad 320$

$\begin{array}{lrl}\text { LFT } & 0 & 2 \\ \text { LFT } & 1 & \\ \text { LFT } & 1 & \\ \text { LFT } & 1 & 3 \\ \text { RLY } & 9 & 11 \\ \text { NOX } & \text { THR } & \end{array}$

C $0-2$

NAI -D

NAI -D

$\begin{array}{lll}D & 1 & \text { NAI I D } \\ \text { C } 1-3 & \text { NAI }-D\end{array}$

D 15,18

$A C T-I$

D $10-28$

C 29-110

$B F 3-I$

C 31

$A C T-I$

D $10-28$

$A C T-I$

C

BF 3-I

EMUI-D

C 12-28

$E M U-D$

c $34-90$

TEL-D

TEL-D

C 34-90

$A C T$ - I

$A C T-I$
ANG REMARKS

DST YIELD REL TO PROTN

4PI MASS SPECTROMETER

90 REL TO PROTONS

* rel to neutrons

90

90 REL TO DEUTERONS

DST * SPC

DST

DST REL TO PROTONS

90 RELATIVE YIELD

90 ABS YIELD

4P I

$100 \mathrm{~J}$

DST LIFETIMES

$110 \mathrm{~J}-\mathrm{PI}$

90

$100 \mathrm{ABI}$

$4 P$ I

4P I

4PI THRESHOLD

4P I

4PI THRESHOLD

4PI THRESHOLD

$4 P$ I

4P I

$4 P I$

4PI * REL TO TRITONS

4P I

4PI MULTIPOLARITIES

4P I

DST REL TO DEUTERONS

DST REL TO PROTONS

DST REL TO PROTONS

4PI * REL TO NEUTRONS

DST SEPARATED ISOTOPE

DST REL TO PROTONS

76 REL TO H2 CROS SEC

100

108 MEAN ABS CROSS SEC

DST MIXING RATIO

$100 A B I$

4PI THRESHOLD

4PI THRESHOLD

4PI REL TO CU63

4P I

$4 P$ I

4PI * REL TO TRITONS

4PI

DST *

DST SPECTRUM

DST REL TO DEUTERONS

DST REL TO PROTONS

4PI * REL TO NEUTRONS $4 P$ I 


\begin{tabular}{|c|c|c|c|c|c|c|c|c|c|}
\hline A & ABUND. & & SEF & AT ION & ENERGIES & (MEV) & & & \\
\hline & & $G, N$ & $G, P$ & $G, T$ & $G, \mathrm{HE} 3$ & $G, A$ & $G, 2 N$ & $G, N P$ & $G, 2 P$ \\
\hline 64 & 48.89 & 11.9 & 7.7 & 19.0 & 16.7 & 4.0 & 21.0 & 18.6 & 13.8 \\
\hline 66 & 27.81 & 11.0 & 8.9 & 18.2 & 18.3 & 4.6 & 19.0 & 18.8 & 16.4 \\
\hline 67 & 4.11 & 7.1 & 8.9 & 17.4 & 15.7 & 4.8 & 18.1 & 16.0 & 17.3 \\
\hline 68 & 18.57 & 10.2 & 10.0 & 17.7 & 19.8 & 5.3 & 17.3 & 19.1 & 18.5 \\
\hline 70 & 0.62 & 9.2 & * & 17.2 & * & 5.9 & 15.7 & 19.5 & $*$ \\
\hline
\end{tabular}

\begin{tabular}{|c|c|c|c|c|c|c|c|c|c|c|}
\hline REF & NUCL IDE & REACT ION & RES & EXCI T & & OURCE & DET & CTOR & ANG & REMARKS \\
\hline & Z A & IN,OUT & & & & IN-MAX & TYPE & I $N-M A X$ & & \\
\hline 6ORE 1 & $30 Z N$ & $G, G$ & $A B X$ & 7 & D & 7 & NAI - D & & 90 & \\
\hline $635 \cup 1$ & $302 N$ & $G, G$ & $A B X$ & 414 & $C$ & $4-14$ & NAI - D & & 120 & \\
\hline $61 T A 1$ & $302 N$ & $G, N$ & NOX & 1222 & $C$ & 22 & THR - I & $6-$ & DST & \\
\hline $56 \mathrm{GA} 1$ & $30 Z N$ & $G, \times N$ & $A B X$ & 727 & $C$ & $9-27$ & $B F 3-I$ & & $4 P I$ & \\
\hline $56 \mathrm{HA} 1$ & $3 C Z N$ & $G, \times N$ & $A B X$ & 15,18 & D & 15,18 & BF 3-I & & $4 P I$ & \\
\hline $64 \mathrm{CO} 2$ & $302 N$ & $G, X N$ & $A B Y$ & THR -80 & $C$ & 80 & $B F 3-I$ & & $4 P I$ & \\
\hline $57 E L 1$ & $30 Z N$ & $G, N P$ & RLX & THR-32 & $C$ & 32 & & & & * REL TO CU \\
\hline $550 L 1$ & $30 Z N$ & $G, P$ & $A B X$ & 18 & D & 18 & $E M U-0$ & & & \\
\hline 56051 & $30 Z N$ & $G, P$ & $S P C$ & THR 31 & $C$ & $20-31$ & $E M U-D$ & & DST & * \\
\hline $63 M I 5$ & $30 Z N$ & $G, \times P$ & $A B Y$ & 1022 & C & 22 & $S C I-I$ & $4-$ & DST & \\
\hline $58 \mathrm{TO} 2$ & $30 Z N$ & $G, A$ & $A B Y$ & 222 & $C$ & 22 & EMU- I & & DST & \\
\hline $550 E 1$ & $30 Z N 64$ & $G \cdot N$ & $A B X$ & 1223 & C & $12-23$ & $A \subset T-I$ & & $4 P I$ & \\
\hline $55 \vee 11$ & $30 Z N 64$ & $G, N$ & $A B X$ & $12-22$ & $C$ & $12-22$ & $A C T-I$ & & $4 \mathrm{P} I$ & \\
\hline 59NAI & $30 Z N 64$ & $G, N$ & $A B X$ & 18 & $D$ & 18 & BF 3-I & & 4PI & \\
\hline 6CRO4 & $302 N 64$ & $G, N$ & $A B X$ & 1223 & $C$ & $12-23$ & $A C T-I$ & & $4 \mathrm{P} \mathrm{I}$ & \\
\hline $61 \mathrm{CO} 2$ & $30 Z N 64$ & $G, N$ & RLX & 15,18 & 0 & 15,18 & $A \subset T-I$ & & $4 P I$ & REL TO CU63 $(\mathrm{G}, \mathrm{N})$ \\
\hline 62DEI & $302 N 64$ & $G, N$ & $A B X$ & 21 & $D$ & 21 & $A C T-I$ & & $4 P I$ & \\
\hline $55 D E 1$ & $30 Z N 64$ & $\mathrm{G}, 2 \mathrm{~N}$ & $A B Y$ & $20 \quad 23$ & C & $20-23$ & $A C T-I$ & & $4 P I$ & THRESHOLD \\
\hline $55 \mathrm{VI} 1$ & $30 Z N 64$ & $G, 2 N$ & $A B Y$ & $15-22$ & $C$ & $12-22$ & $A C T-I$ & & 4P I & \\
\hline $58 \mathrm{HO} 1$ & $30 Z N 64$ & $G, 2 N$ & $A B I$ & 2028 & C & 28 & $A C T-I$ & & $4 P I$ & THRESHOLD \\
\hline 58603 & $30 Z N 64$ & $G, N P$ & $A B X$ & 1622 & C & $16-22$ & $A \subset T-I$ & & 4PI & REACTION UNCERTAIN \\
\hline $58 \mathrm{HO} 1$ & $30 Z N 64$ & $G, N P$ & $A B I$ & 1828 & $C$ & 28 & $A C T-I$ & & 4P I & THRESHOLD \\
\hline $58 \mathrm{GO} 3$ & $30 Z N 64$ & $G, D$ & $A B X$ & $16 \quad 22$ & C & $16-22$ & $A C T-I$ & & 4P I & REACTION UNCERTAIN \\
\hline $550 E_{1}$ & $30 Z N 66$ & $G, N P$ & $A B X$ & 1923 & C & $19-23$ & $A \subset T-I$ & & $4 \mathrm{PI}$ & \\
\hline 58603 & $30 Z N 66$ & $G, N P$ & $A B X$ & 1622 & C & $16-22$ & $A \subset T-I$ & & 4PI & REACTION UNCERTA IN \\
\hline $58 \mathrm{HO}$ & $30 Z N 66$ & $G, N P$ & $A B X$ & 2132 & $C$ & $15-32$ & $A C T-I$ & & $4 P I$ & THRESHOLD \\
\hline $55 \mathrm{VII}$ & $30 Z N 66$ & $G, D$ & $A B X$ & $20-22$ & C & $20-22$ & $A C T-I$ & & 4P I & INCLUDES G,NP \\
\hline $58 \mathrm{GO} 3$ & $3 C Z N 66$ & $G, D$ & $A B X$ & 1622 & C & $16-22$ & $A C T-I$ & & $4 \mathrm{PI}$ & REACTION UNCERTAIN \\
\hline $58 \mathrm{HO} 1$ & $30 Z N 68$ & $G, P$ & RLY & 1032 & $C$ & $15-32$ & $A C T-I$ & & 4PI & THRESHOLD \\
\hline
\end{tabular}




\begin{tabular}{|c|c|c|c|c|c|c|c|c|c|}
\hline A & ABUND. & & SE & $\triangle T I O N$ & ENERGIES & (MEV) & & & \\
\hline 69 & 60.4 & $\begin{array}{r}G, N \\
10.3\end{array}$ & $\begin{array}{l}G, P \\
6.6\end{array}$ & $\begin{array}{r}G, T \\
15.4\end{array}$ & $\begin{array}{l}\mathrm{G}, \mathrm{HE} 3 \\
18.0\end{array}$ & $\begin{array}{l}G, A \\
4.5\end{array}$ & $\begin{array}{l}G, 2 N \\
16.6\end{array}$ & $\begin{array}{l}G, N P \\
16.9\end{array}$ & $\begin{array}{l}G, 2 P \\
16.6\end{array}$ \\
\hline 71 & 39.6 & 9.3 & 7.9 & 15.1 & 19.7 & 5.3 & 17.0 & 17.1 & * \\
\hline
\end{tabular}

\begin{tabular}{|c|c|c|c|c|c|c|c|c|c|}
\hline REF & $\underset{Z}{N U C L}$ IDE & $\begin{array}{l}\text { REACT ION } \\
\text { IN,OUT }\end{array}$ & RES & EXCIT & $\begin{array}{l}\text { SOURCE } \\
\text { MIN-MAX }\end{array}$ & $\begin{array}{c}\text { DETECTOR } \\
\text { TYPE MIN-MAX }\end{array}$ & ANG & REMARKS & \\
\hline $64 \mathrm{CO} 2$ & $31 \mathrm{GA}$ & $G, \times N$ & $A B Y$ & $T H R-80$ & C 80 & $B F 3-I$ & $4 \mathrm{PI}$ & & \\
\hline $59 C A 1$ & $31 G A 66$ & $D, G$ & $A B X$ & 1416 & $3-5$ & $A C T-I$ & 4P I & NOT GRND STAT & TRAN \\
\hline $60 \mathrm{GE} 3$ & $31 G A 71$ & $G, N$ & NOX & THR & CTHR & $B F 3-I$ & $4 \mathrm{PI}$ & THRESHOLD & \\
\hline
\end{tabular}

GERMANIUM $Z=32$

\begin{tabular}{|c|c|c|c|c|c|c|c|c|c|}
\hline A & ABUND. & & SEI & ATION & ENERGIES & (MEV) & & & \\
\hline & & $G, N$ & $G, P$ & $G, T$ & G,HE3 & $G, A$ & $G, 2 N$ & $G, N P$ & $G, 2 P$ \\
\hline 70 & 20.52 & 11.5 & 8.5 & 18.6 & 17.6 & 4.1 & 20. & 18.9 & 15.1 \\
\hline 72 & 27.43 & 10.7 & 9.7 & 18.2 & 19.1 & 5.0 & 18.2 & 19.1 & 17.6 \\
\hline 73 & 7.76 & 6.8 & 10.0 & 17.3 & 16.7 & 5.3 & 17.5 & 16.5 & 18.4 \\
\hline 74 & 36.54 & 10.1 & 11.0 & 18.2 & 20.8 & 6.3 & 17.0 & 20.2 & 19.9 \\
\hline 76 & 7.76 & 9.4 & * & 18.4 & * & 7.5 & 16.9 & 20.8 & \\
\hline
\end{tabular}

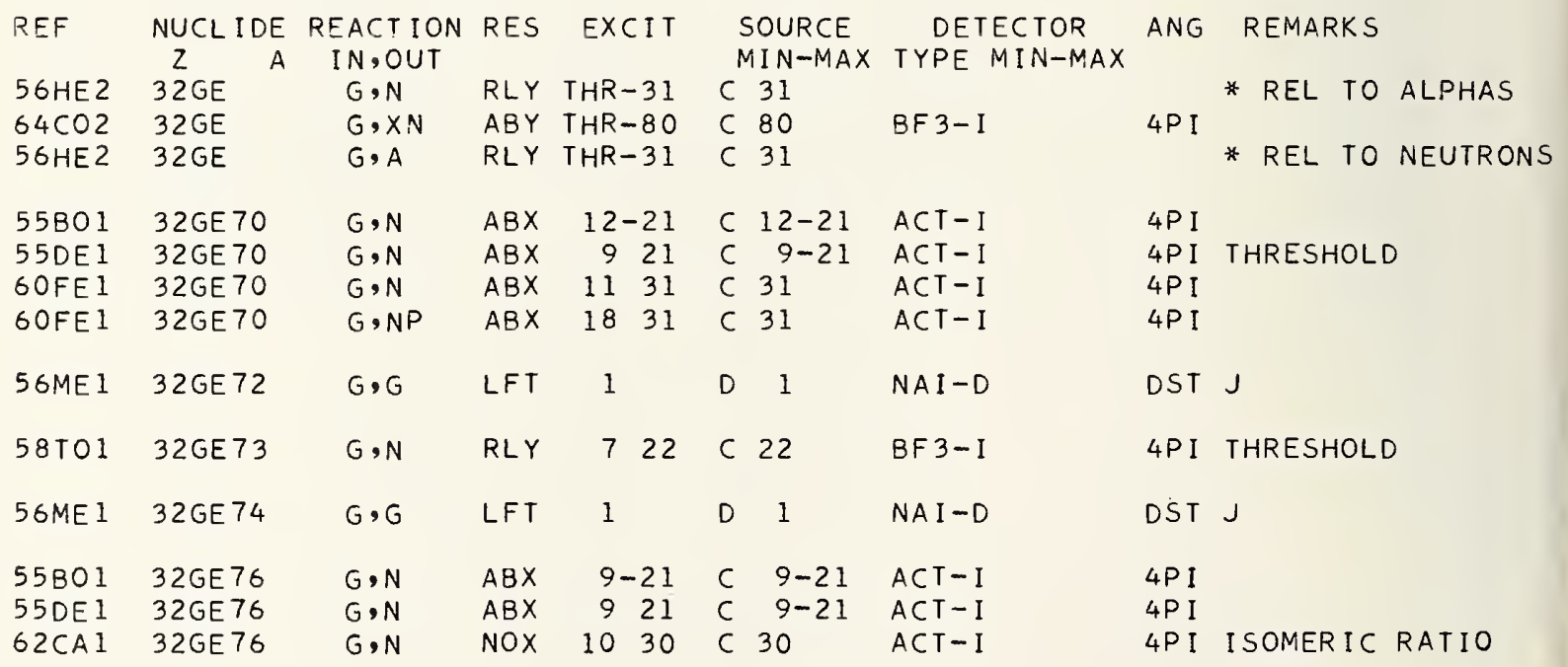




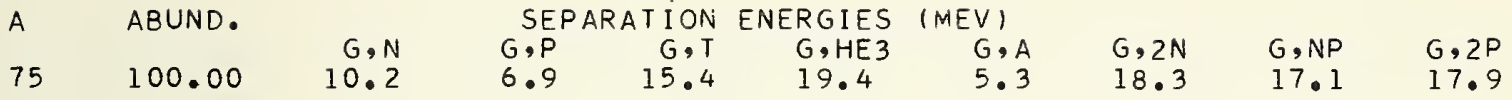

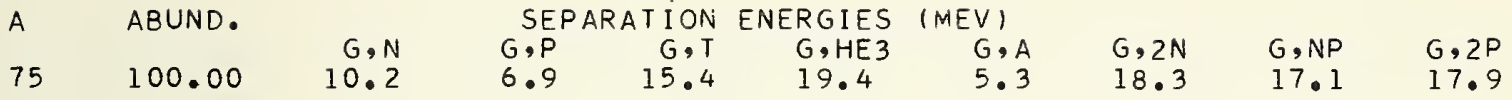

REF NUCLIDE REACTION RES EXCIT SOURCE DETECTOR ANG REMARKS

\begin{tabular}{|c|c|c|c|c|c|c|c|c|c|}
\hline & Z & IN, OUT & & & MIN-MAX & TYPE N & IN-N & & \\
\hline 62ME2 & $33 A 575$ & $G, G$ & LFT & 1 & D & NAI-D & & 0 & \\
\hline 635U1 & 33 AS 75 & $G, G$ & $A B X$ & $4 \quad 14$ & $4-14$ & $N A I-D$ & & 120 & \\
\hline $56 S_{1}$ & 33 AS 75 & $G, N$ & RLY & THR -320 & $\mathrm{C} 140,320$ & $A C T-I$ & & 4PI & * \\
\hline 57FE2 & 33 AS 75 & $G, N$ & $A B Y$ & 1430 & C $14-30$ & THR-I & $5-$ & $4 \mathrm{PI}$ & \\
\hline $58 \mathrm{CH} 2$ & 33 AS 75 & $G, N$ & RLY & THR & CTHR & $B F 3-I$ & & 4PI & THRESHOLD \\
\hline 60GE 3 & 33 AS 75 & $G, N$ & NOX & THR & CTHR & BF 3-I & & 4PI & THRESHOLD \\
\hline $61 \mathrm{TA} 1$ & $33 A . S 75$ & $G, N$ & NOX & 722 & C 22 & THR-I & $6-$ & DST & \\
\hline 56SU1 & 33 AS 75 & $G, 3 N$ & RLY & THR -320 & $C 140,320$ & $A C T-I$ & & $4 P I$ & * \\
\hline $58 \mathrm{KAl}$ & $33 A S 75$ & $G, \times N$ & $A B X$ & 1022 & C $10-22$ & BF 3-I & & 4PI & \\
\hline $61 B A 2$ & 33 AS 75 & $G, \times N$ & $A B Y$ & THR 22 & C 22 & THR - I & $3-$ & DST & \\
\hline $61 B A 2$ & 33 AS 75 & $G, \times N$ & $A B Y$ & THR 22 & C 22 & THR-I & $5-$ & DST & \\
\hline $64 \mathrm{CO2}$ & 33 AS 75 & $G, \times N$ & $A B Y$ & THR-80 & C 80 & $B F 3-I$ & & $4 P I$ & \\
\hline U1 & 33 AS 75 & $G, N 2 P$ & RLY & THR-320 & $C 140,320$ & $A C T-I$ & & 4PI & * \\
\hline 56 & $33 A 575$ & $G, 3 N 2 P$ & RLY & THR-320 & $C 140,320$ & $A C T-I$ & & $4 \mathrm{PI}$ & * \\
\hline $56 \mathrm{SU}_{1}$ & 33 AS75 & $G, 4 N 4 P$ & RLY & THR -320 & $C 140,320$ & $A C T-I$ & & $4 \mathrm{PI}$ & * \\
\hline 56SU1 & $33 A S 75$ & $G, 4 N 5 P$ & RLY & THR-320 & $\mathrm{C} 140,320$ & $A C T-I$ & & $4 P I$ & * \\
\hline & $33 A S 75$ & $G, 5 N 2 P$ & RLY & THR-320 & $C 140,320$ & $A C T-I$ & & 4PI & * \\
\hline 56SU1 & 33 AS 75 & $G, 5 N 5 P$ & $R L Y$ & THR-320 & $\mathrm{C} 140,320$ & $A C T-I$ & & 4PI & * \\
\hline 56SU1 & $33 A S 75$ & $G, 7 N 2 P$ & RLY & THR -320 & $C 140,320$ & $A C T \rightarrow I$ & & 4PI & * \\
\hline 56SU1 & 33 AS 75 & $G, 7 N 4 P$ & $R L Y$ & THR -320 & $C 140,320$ & $A C T-I$ & & $4 \mathrm{PI}$ & * \\
\hline 56SU1 & 33 AS 75 & $G, 8 N 6 P$ & RLY & $T H R-320$ & $C 140,320$ & $A C T-I$ & & 4P I & * \\
\hline $56 \mathrm{SU} 1$ & 33 AS 75 & $G, 13 N 4$ & RLY & THR-320 & $C 140,320$ & $A C T-I$ & & $4 P I$ & * G, $13 N 4 P$ \\
\hline 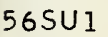 & 33 AS 75 & $G, 14 N 6$ & RLY & THR-320 & $(140,320$ & $A C T-I$ & & 4P I & * G, 14N6P \\
\hline$U_{1}$ & 33 AS 75 & $G, 2 P$ & $R L Y$ & THR-320 & $C 140,320$ & $A C T-I$ & & 4PI & * \\
\hline
\end{tabular}

SELENIUM $Z=34$

\begin{tabular}{|c|c|c|c|c|c|c|c|c|c|}
\hline A & ABUND. & & SE & ATION & ENERGIES & (MEV) & & & \\
\hline & 0.87 & $\mathrm{G}, \mathrm{N}$ & $\begin{array}{l}G, P \\
8.6\end{array}$ & $\begin{array}{r}\text { G.T } \\
9.3\end{array}$ & $\begin{array}{l}\text { G,HE3 } \\
17.2\end{array}$ & $\begin{array}{l}G, A \\
4.1\end{array}$ & $\begin{array}{l}\mathrm{G}, 2 \mathrm{~N} \\
20.7\end{array}$ & $\begin{array}{l}G, N P \\
19.4\end{array}$ & $\begin{array}{l}G, 2 P \\
14.2\end{array}$ \\
\hline 76 & 9.02 & 11.2 & 9.5 & 19.3 & 18.9 & 5.1 & 19.2 & 19.8 & 16.4 \\
\hline 77 & 7.58 & 7.4 & 9.6 & 18.7 & 16.1 & 5.7 & 18.6 & 17.0 & 17.3 \\
\hline 78 & 23.52 & 10.5 & 10.4 & 18.9 & $20 \cdot 1$ & 6.0 & 17.9 & 20.1 & 18.4 \\
\hline $\begin{array}{l}80 \\
82\end{array}$ & $\begin{array}{r}49.82 \\
9.19\end{array}$ & $\begin{array}{l}9.9 \\
9.3\end{array}$ & 11.4 & $\begin{array}{l}18.7 \\
18.8\end{array}$ & 21.5 & $\begin{array}{l}7.0 \\
*\end{array}$ & $\begin{array}{l}16.9 \\
16.0\end{array}$ & $\begin{array}{l}20.4 \\
21.2\end{array}$ & * \\
\hline
\end{tabular}

\begin{tabular}{|c|c|c|c|c|c|c|c|c|c|c|}
\hline REF & $\underset{2}{\text { NUCL IDE }}$ & $\begin{array}{l}\text { REACT I ON } \\
\text { IN, OUT }\end{array}$ & RES & $E \times C$ & IT & & $\begin{array}{l}\text { OURCE } \\
\text { IN-MAX }\end{array}$ & $\begin{array}{c}\text { DETECTOR } \\
\text { TYPE MIN-MAX }\end{array}$ & ANG & REMARKS \\
\hline $\begin{array}{l}\text { 6ODE2 } \\
\text { 63KA2 } \\
63 \text { VE2 }\end{array}$ & $\begin{array}{l}34 \mathrm{SE} \\
34 \mathrm{SE} \\
34 \mathrm{SE}\end{array}$ & $\begin{array}{l}G, G \\
G, G / \\
G, G\end{array}$ & $\begin{array}{l}\text { LFT } \\
R L Y \\
A B X\end{array}$ & $\begin{array}{l}1 \\
1 \\
0\end{array}$ & 1 & $\begin{array}{l}\text { D } \\
C \\
D\end{array}$ & $\begin{array}{l}1 \\
5 \\
0-1\end{array}$ & $\begin{array}{l}A C T-I \\
N A I-D\end{array}$ & 4P I & $\begin{array}{l}\text { * } \\
\text { I SOMERS }\end{array}$ \\
\hline 63PR2 & 34 SE 76 & $G, G$ & LFT & 1 & & D & 1 & NA I -D & & \\
\hline $58 \mathrm{HOI}$ & 34 SE 80 & $G, N P$ & RLY & 20 & 32 & $c$ & 32 & $A C T-I$ & $4 P I$ & THRESHOLD \\
\hline $6 S I 2$ & 34 SE 82 & $G, N$ & $A B X$ & & -22 & C & $9-22$ & $A C T-I$ & 4P I & \\
\hline
\end{tabular}


Sr

\begin{tabular}{|c|c|c|c|c|c|c|c|c|c|}
\hline A & ABUND. & & 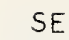 & TION & ENERGIES & (MEV) & & & \\
\hline 79 & 50.54 & $\begin{array}{r}G, N \\
10.7\end{array}$ & $\begin{array}{l}G, P \\
6.3\end{array}$ & $\begin{array}{r}G, T \\
15.8\end{array}$ & $\begin{array}{l}\text { G, HE } 3 \\
18.7\end{array}$ & $\begin{array}{l}5 . A \\
5.5\end{array}$ & $\begin{array}{l}G, 2 N \\
19.0\end{array}$ & $\begin{array}{l}G, N P \\
16.9\end{array}$ & $\begin{array}{l}G, 2 P \\
16.7\end{array}$ \\
\hline 81 & 49.46 & $10 \cdot 2$ & 7.5 & 15.9 & 20.2 & 6.5 & 18.0 & 17.4 & 18.9 \\
\hline
\end{tabular}

\begin{tabular}{|c|c|c|c|c|c|c|c|c|}
\hline REF & NUCL IDE & REACTION & RES & EXC I T & SOURCE & DETECTOR & $A M G$ & REMARKS \\
\hline & A & IN,OUT & & & MIN-MAX & TYPE MIN-MAX & & \\
\hline $63 \mathrm{KA} 2$ & $35 B R$ & $G, G /$ & RLY & 1 & C & $A C T-I$ & 4P I & \\
\hline $7 K I 1$ & $35 \mathrm{BR} 81$ & $G, N$ & RLY & 1070 & C 15-70 & $B F 3-I$ & $4 P I$ & \\
\hline OGE3 & $35 B R 81$ & $G, N$ & NOX & THR & CTHR & $B F 3-I$ & 4P I & THRESHOLD \\
\hline A 1 & $35 B R 81$ & $G, N$ & NOX & 1030 & C 30 & $A C T-I$ & $4 P I$ & I SOMER I C RATI \\
\hline$C 2$ & $35 \mathrm{BR} 81$ & $G, A$ & $A B X$ & $6-32$ & C 32 & $A \subset T-I$ & 4P I & * \\
\hline R 1 & 35 BR 81 & $G, A$ & $A B I$ & $6 \quad 32$ & C 32 & $A C T-I$ & $4 \mathrm{PI}$ & \\
\hline
\end{tabular}

\begin{tabular}{|c|c|c|c|c|c|c|c|c|c|}
\hline A & ABUND. & & SEF & TION & ENERGIES & (MEV) & & & \\
\hline & & $G, N$ & $G, P$ & $G, T$ & $G, H E 3$ & $G, A$ & $G, 2 N$ & $G, N P$ & $G, 2 P$ \\
\hline 85 & 72.15 & 10.5 & 7.0 & 16.5 & 19.6 & 6.6 & 19. & 17.6 & 17.7 \\
\hline 87 & 27.85 & 9.9 & 8.6 & 17.1 & 21.8 & 8.0 & $18 \cdot 6$ & 18.5 & $20 \cdot 5$ \\
\hline
\end{tabular}

REF NUCLIDE REACTION RES EXCIT SOURCE DETECTOR ANG REMARKS

\begin{tabular}{|c|c|c|c|c|c|c|c|c|c|c|}
\hline & $z$ & IN,OUT & & & & MIN-MAX & TYPE & I N-MAX & & \\
\hline $61 B A 2$ & $37 R B$ & $G, \times N$ & $A B Y$ & THR & 22 & C 22 & THR - I & $5-$ & DST & \\
\hline $\begin{array}{l}8 T O 1 \\
\text { OGE3 }\end{array}$ & $\begin{array}{l}37 R B 85 \\
37 R B 85\end{array}$ & $\begin{array}{l}G, N \\
G, N\end{array}$ & $\begin{array}{l}\text { RLY } \\
\text { NOX }\end{array}$ & $\begin{array}{r}10 \\
\text { THR }\end{array}$ & 11 & $\begin{array}{l}\text { C } 22 \\
\text { CTHR }\end{array}$ & $\begin{array}{l}A C T-I \\
B F 3-I\end{array}$ & & $\begin{array}{l}\text { 4P I } \\
4 \mathrm{PI}\end{array}$ & $\begin{array}{l}\text { THRESHOLD } \\
\text { THRESHOLD }\end{array}$ \\
\hline $\begin{array}{l}8 T O 1 \\
\text { OGE } 3\end{array}$ & $\begin{array}{l}37 R B 87 \\
37 R B 87\end{array}$ & $\begin{array}{l}G, N \\
G, N\end{array}$ & $\begin{array}{l}\text { RLY } \\
\text { NOX }\end{array}$ & $\begin{array}{r}9 \\
\text { THR }\end{array}$ & 11 & $\begin{array}{l}\text { C } 22 \\
\text { CTHR }\end{array}$ & $\begin{array}{l}B F 3-I \\
B F 3-I\end{array}$ & & $\begin{array}{l}4 P I \\
4 P I\end{array}$ & $\begin{array}{l}\text { THRESHOLD } \\
\text { THRESHOLD }\end{array}$ \\
\hline $7 E R 1$ & 37 RB 87 & $G, N A$ & AB I & 17 & 32 & C 32 & $A C T-I$ & & 4P I & \\
\hline 7ER I & $37 R B 87$ & $G, A$ & $A B I$ & 8 & 32 & C 32 & $A C T-I$ & & 4P I & \\
\hline
\end{tabular}

$$
\text { STRONTIUM } Z=38
$$

A ABUND.

84

86

87

88

$\begin{array}{rr}0.56 & 12 . \\ 9.86 & 11.5 \\ 7.02 & 8.4 \\ 82.56 & 11.1\end{array}$

SEPARATION ENERGIES (MEV)

$\begin{array}{llllll}\text { G.P } & \text { G.T } & \text { G.HE3 } & \text { G.A } & \text { G. } 2 N & \text { G.NP } \\ 8 . & 20.2 & 17.9 & 5.2 & 21 . & 19.6 \\ 9.6 & 20 . & 19.4 & 6.3 & 20.0 & 20.1 \\ 9.4 & 20.1 & 17.4 & 7.3 & 20.0 & 18.1 \\ 10.6 & 20.7 & 21.3 & 7.9 & 19.5 & 20.6\end{array}$

G. 2P

14.6

16.6

18.0

19.2

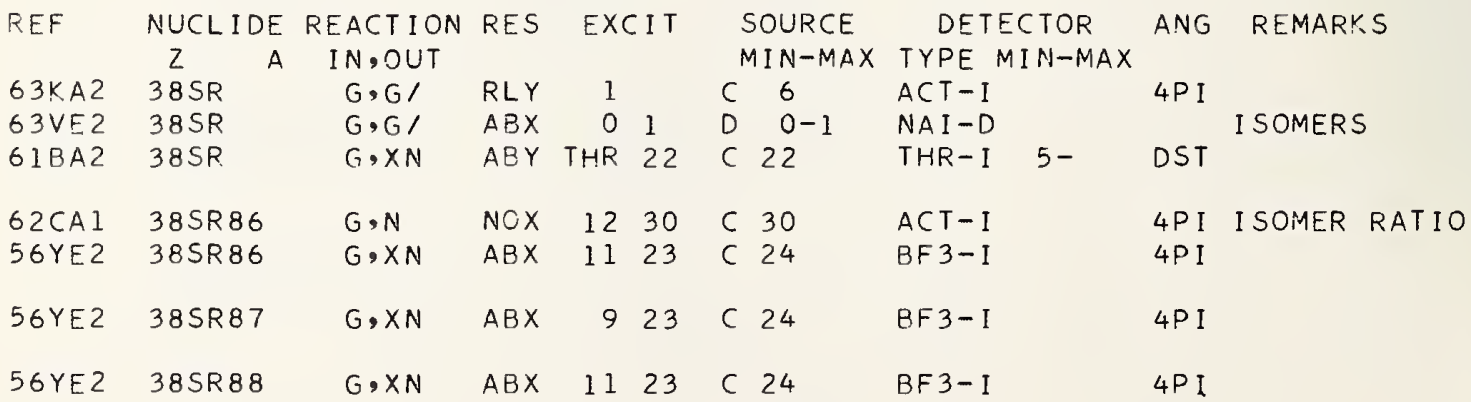




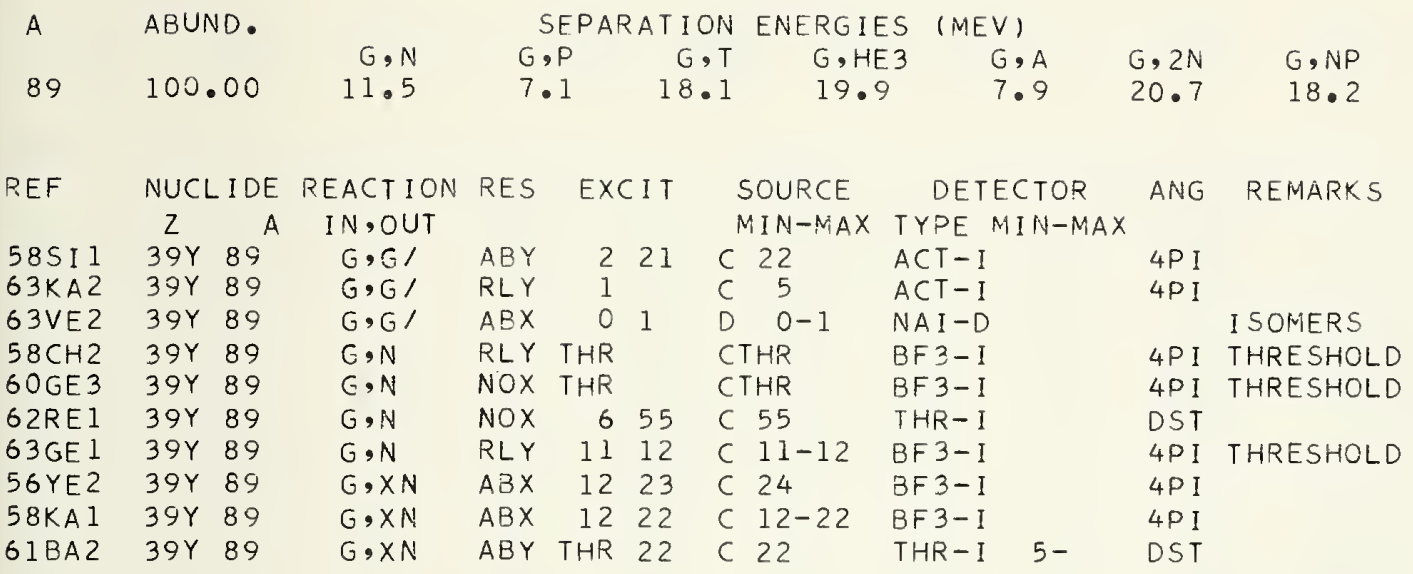

\section{ZIRCONIUM $Z=40$}

\begin{tabular}{|c|c|c|c|c|c|c|c|c|c|}
\hline A & ABUND. & & SEF & ATION & ENERGIES & $(M E V)$ & & & \\
\hline & & $G, N$ & $G, P$ & $G, T$ & G, HE3 & $G, A$ & $\mathrm{G}, 2 \mathrm{~N}$ & $G, N P$ & $G, 2 P$ \\
\hline 90 & 51.46 & 12.0 & 8.4 & 20.6 & 18.8 & 6.7 & 21. & 19.9 & 15.5 \\
\hline 91 & 11.23 & 7.2 & 8.7 & 18.6 & 14.9 & 5.4 & 19.2 & 15.6 & 16. \\
\hline 92 & 17.11 & 8.6 & $9 \cdot 4$ & 15.7 & 17.2 & 3.0 & 15.8 & 17.4 & 7. \\
\hline 94 & 17.40 & $8 \cdot 2$ & 10.3 & 15.9 & $18 \cdot 5$ & 3.8 & 14.9 & 17.8 & 8 . \\
\hline 96 & 2.80 & 7.8 & 11 . & 16.1 & 20.9 & 4.9 & $14 \cdot 3$ & $18 \cdot 5$ & 1 . \\
\hline
\end{tabular}

\begin{tabular}{|c|c|c|c|c|c|c|c|c|c|c|c|}
\hline & $z$ & IN,OUT & & & & & IIN-MAX & TYPE & I N-MAX & & \\
\hline 6ORE 1 & $40 Z R$ & $G, G$ & $A B X$ & 7 & & D & 7 & $N A I-D$ & & 90 & \\
\hline 64DU1 & $40 Z R$ & $G, P$ & $S P C$ & THR- & 34 & C & $22-34$ & $M A G-D$ & $2-16$ & DST & $A B X$ \\
\hline 63 MI 5 & $40 Z R$ & $G, X P$ & $A B Y$ & 8 & 22 & C & 22 & $S C I-I$ & $4-$ & DST & \\
\hline $63 \mathrm{~A} \times 1$ & 40ZR 90 & $G, G$ & $A B X$ & 8 & 13 & D & $8-13$ & NAI $-D$ & & 135 & \\
\hline $56 \mathrm{~A} \times 1$ & 40ZR 90 & $G, N$ & RLX & 12 & 23 & C & $12-23$ & $A C T-I$ & & 4PI & THRESHOLD \\
\hline $59 M U 2$ & 40ZR 90 & $G, N$ & $R L X$ & 12 & 24 & C & $12-25$ & $A C T-I$ & & $4 P I$ & \\
\hline $62 C A 1$ & $40 Z R 90$ & $G, N$ & NOX & 12 & 30 & C & 30 & $A C T-I$ & & $4 \mathrm{PI}$ & I SOMER IC RATIO \\
\hline 56YE2 2 & 40ZR 90 & $G, \times N$ & $A B X$ & 12 & 23 & c & 24 & $B F 3-I$ & & $4 \mathrm{PI}$ & \\
\hline 59 NA 1 & 40ZR 90 & $G, X N$ & $A B X$ & 12 & 24 & $c$ & $5-24$ & $B F 3-I$ & & $4 \mathrm{PI}$ & THRESHOLD \\
\hline $64 B L 2$ & $40 Z R 90$ & $P, G$ & RLX & $13-$ & 17 & D & $5-9$ & $N A I-D$ & & DST & ANALOGUE $T=6$ \\
\hline $55 \mathrm{NA} 1$ & 40ZR 91 & $G, \times N$ & $A B X$ & 7 & 24 & $c$ & $5-24$ & $B F 3-I$ & & $4 P I$ & THRESHOLD \\
\hline 56 YE2 & $402 R 91$ & $G, X N$ & $A B X$ & 7 & 23 & c & 24 & $B F 3-I$ & & $4 P I$ & \\
\hline SYE 2 & 40ZR 92 & $G, \times N$ & $A B X$ & 9 & 23 & $c$ & 24 & BF 3-I & & $4 \mathrm{PI}$ & \\
\hline
\end{tabular}


A ABUND. SEPARATION ENERGIES (MEV)

93100.00

$\mathrm{G}, \mathrm{N}$

$G, P \quad G, T$

$\mathrm{G}, \mathrm{HE} 3$

$G, A$

$\mathrm{G}, 2 \mathrm{~N}$

$G, N P$

G, 2P

6. 013.4

15.7

2. 0

16.6

14.7

15.4

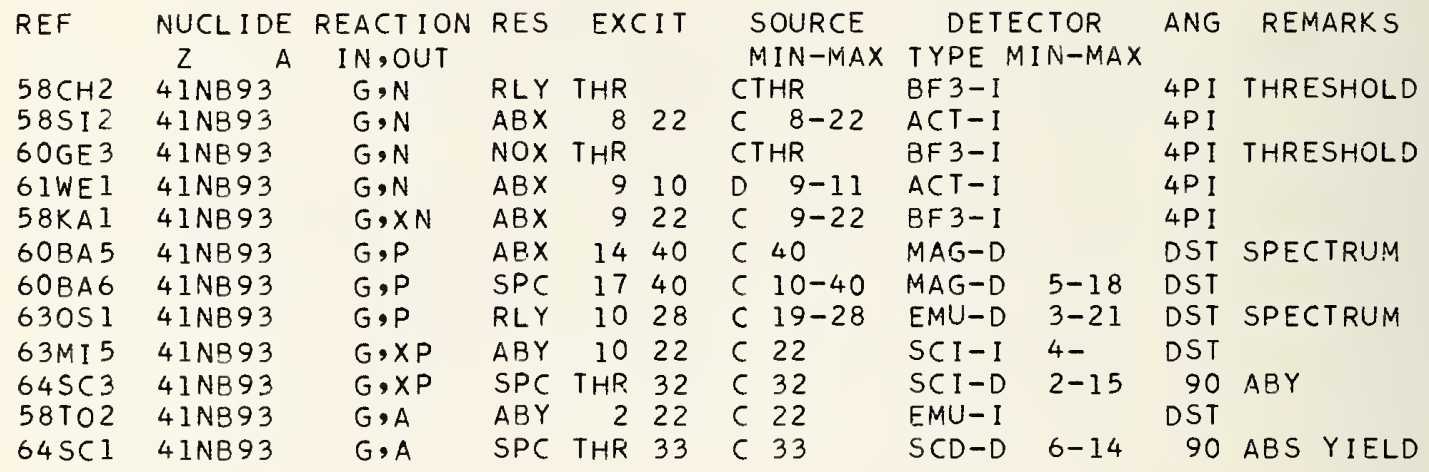

MOLYBDENUM $Z=42$

\begin{tabular}{|c|c|c|c|c|c|c|c|c|c|}
\hline A & ABUND. & & $\mathrm{SE}$ & ATION & ENERG IFS & (MEV) & & & \\
\hline & & $G, N$ & $G, P$ & $G, T$ & $\mathrm{G}, \mathrm{HE} 3$ & $G, A$ & $\mathrm{G}, 2 \mathrm{~N}$ & $G, N P$ & $G, 2$ \\
\hline 92 & 15.84 & 12.6 & $7 \cdot 3$ & 20.8 & 16.9 & 5. & 22.8 & 19.5 & 6 \\
\hline 94 & 9.04 & 9.7 & 8.5 & 16.6 & 15.4 & 2.1 & 17.7 & 17.4 & 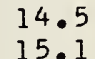 \\
\hline $\begin{array}{l}95 \\
96\end{array}$ & 15.72 & 7.4 & 8.7 & 16.2 & 14.2 & 2.2 & 17.1 & 15.9 & \\
\hline $\begin{array}{l}96 \\
97\end{array}$ & $\begin{array}{r}16.53 \\
9\end{array}$ & $\begin{array}{l}9.2 \\
6.8\end{array}$ & 9.3 & 16.5 & $16 \cdot 6$ & 2.8 & 16.5 & 17.8 & $\begin{array}{l}16 . \\
16 .\end{array}$ \\
\hline $\begin{array}{l}91 \\
98\end{array}$ & $\begin{array}{r}9.46 \\
23.78\end{array}$ & $\begin{array}{l}6.8 \\
8.6\end{array}$ & $\begin{array}{l}9.2 \\
9.8\end{array}$ & $\begin{array}{l}16.1 \\
16.3\end{array}$ & $\begin{array}{l}15.2 \\
17.4\end{array}$ & $\begin{array}{l}2.8 \\
3.3\end{array}$ & $\begin{array}{l}16.0 \\
15.5\end{array}$ & $\begin{array}{l}16.1 \\
17.9\end{array}$ & 7. \\
\hline 100 & 9.63 & $8 \cdot 3$ & 11. & 15.5 & $18 \cdot 2$ & 3.2 & 14.2 & 18. & \\
\hline
\end{tabular}

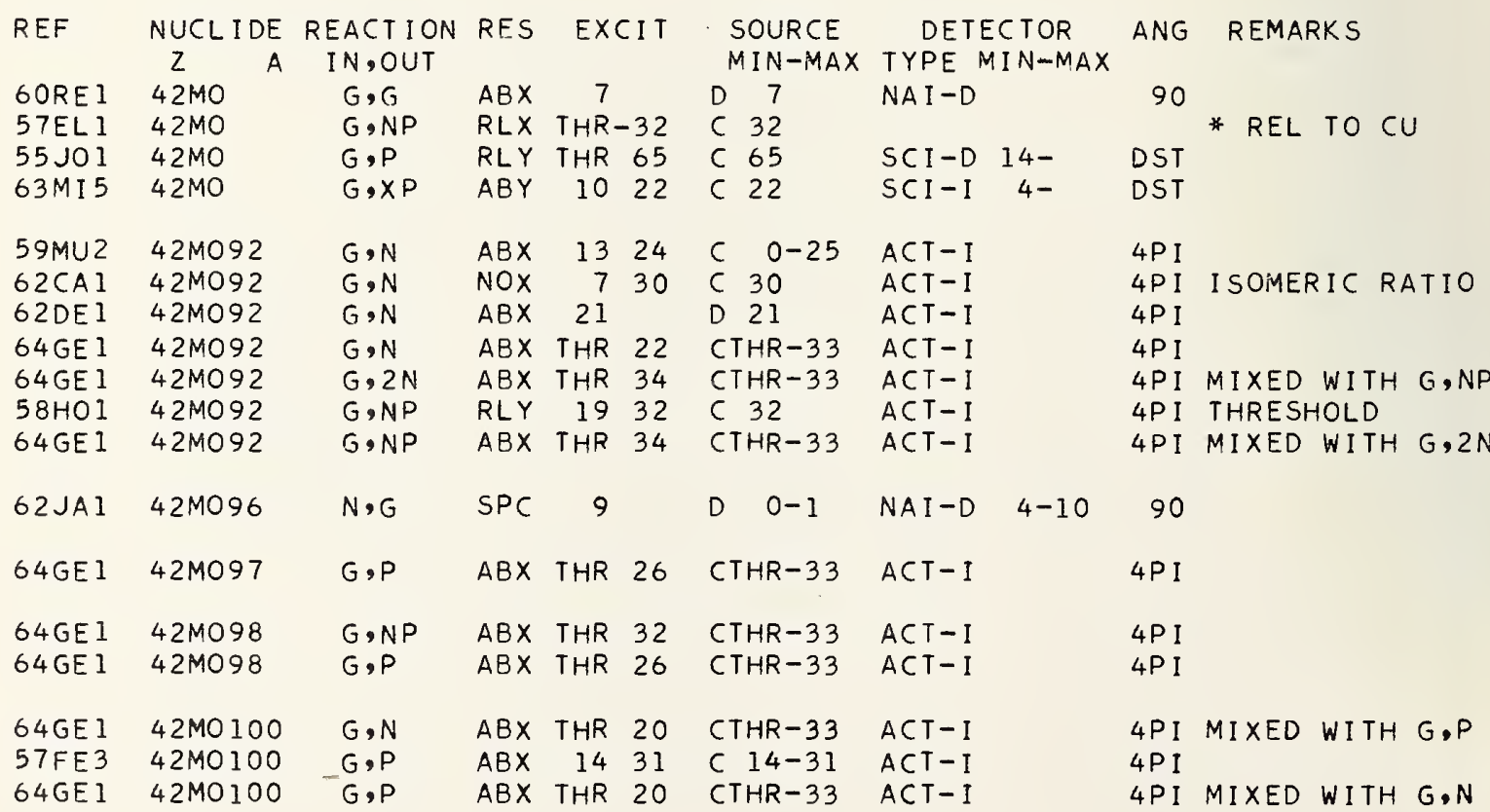




\begin{tabular}{|c|c|c|c|c|c|c|c|c|}
\hline A & BUND. & & SE & TION & ENERGIES & (MEV) & & \\
\hline 103 & 100.00 & $\begin{array}{l}G, N \\
9.3\end{array}$ & $\begin{array}{l}G, P \\
6.2\end{array}$ & $\begin{array}{r}G, T \\
13.9\end{array}$ & $\begin{array}{l}\text { G, HE } 3 \\
15.5\end{array}$ & $\begin{array}{l}G, A \\
3.1\end{array}$ & $\begin{array}{l}G, 2 N \\
16.8\end{array}$ & $\begin{array}{l}G, N P \\
15.4\end{array}$ \\
\hline
\end{tabular}

\begin{tabular}{|c|c|c|c|c|c|c|c|c|c|c|c|c|}
\hline \multirow[t]{2}{*}{ REF } & \multirow{2}{*}{$\begin{array}{cc}\text { NUCL IDE } \\
Z\end{array}$} & \multirow{2}{*}{$\begin{array}{l}\text { REACT I ON } \\
\text { IN, OUT }\end{array}$} & \multirow[t]{2}{*}{ RES } & \multirow{2}{*}{\multicolumn{2}{|c|}{ EXCIT }} & \multirow{2}{*}{\multicolumn{2}{|c|}{$\begin{array}{l}\text { SOURCE } \\
\text { MIN-MAX }\end{array}$}} & \multicolumn{2}{|c|}{ DETECTOR } & \multirow[t]{2}{*}{ ANG } & \multicolumn{2}{|l|}{ REMARKS } \\
\hline & & & & & & & & TYPE $N$ & IN-MAX & & & \\
\hline $\begin{array}{l}59 \text { IKI } \\
60 \mathrm{BO} 2\end{array}$ & $\begin{array}{l}45 \mathrm{RH} 103 \\
45 \mathrm{RH} 103\end{array}$ & $\begin{array}{l}G, G / \\
G, G /\end{array}$ & $\begin{array}{l}A B X \\
A B X\end{array}$ & $\begin{array}{l}1- \\
6\end{array}$ & $\begin{array}{l}2 \\
26\end{array}$ & D & $\begin{array}{l}1-2 \\
6-26\end{array}$ & $\begin{array}{l}A C T-I \\
A C T-I\end{array}$ & & $\begin{array}{l}\text { 4P I } \\
4 \mathrm{PI}\end{array}$ & * & \\
\hline 63VE2 & $45 \mathrm{RHIO}_{3}$ & $G, G /$ & $A B X$ & 0 & 1 & $D$ & $0-1$ & NAI -D & & & I SOMERS & \\
\hline $58 \mathrm{CH} 2$ & $45 \mathrm{RH}_{103}$ & $G, N$ & RLY & THR & & $C T$ & HR & BF 3-I & & 4PI & THRESHOLD & \\
\hline 59PA2 & $45 \mathrm{RH} 103$ & $G, N$ & $A B X$ & 9 & 24 & $C$ & $9-24$ & BF 3-I & & 4PI & QUADRUPOLE & MOMENT \\
\hline $60 G E 3$ & $45 \mathrm{RH} 103$ & $G, N$ & NOX & THR & & & $H R$ & BF 3-I & & 4P I & THRESHOLD & \\
\hline $62 \mathrm{BO} 1$ & $45 \mathrm{RH} 103$ & $G, N$ & $A B X$ & 10 & 23 & $C$ & $10-23$ & BF 3-I & & $4 P I$ & & \\
\hline $58 \mathrm{KAl}$ & $45 \mathrm{RH} 103$ & $G, \times N$ & $A B X$ & 9 & 22 & $C$ & $9-22$ & BF 3-I & & 4PI & & \\
\hline $59 A G I$ & $45 \mathrm{RH} 103$ & $G, \times N$ & $S P C$ & & -30 & $c$ & 30 & & & & * SURFACE & EFFECTS \\
\hline 550A I & $45 \mathrm{RHIO} 3$ & $G, P$ & $S P C$ & THR- & -70 & $c$ & 70 & $E M U-D$ & -16 & & * & \\
\hline $625 \mathrm{H}_{2}$ & $45 \mathrm{RH} 103$ & $G, P$ & $A B Y$ & 9 & 34 & $c$ & 23,34 & $E M U-D$ & $3-24$ & DST & SPECTRUM & \\
\hline $62 \mathrm{SH}_{4}$ & $45 \mathrm{RH} 103$ & $G, P$ & $A B Y$ & 9 & 34 & $C$ & 23,34 & EMU-D & $3-24$ & DST & SPECTRUM & \\
\hline $56 D A 2$ & $45 \mathrm{RH} 103$ & $G, X P$ & $A B Y$ & 10 & 70 & $c$ & 70 & $E M U-D$ & $4-16$ & DST & SPECTRUM & \\
\hline 63152 & $45 \mathrm{RH} 103$ & $G, \times P$ & $A B X$ & 13 & 32 & $c$ & $14-32$ & $S C I-D$ & $3-$ & DST & & \\
\hline $58 \mathrm{TO} 2$ & $45 \mathrm{RH} 103$ & $G, A$ & $A B Y$ & THR & 22 & $c$ & 22 & $E M U-I$ & & DST & & \\
\hline
\end{tabular}

PALLADIUM $Z=46$

\begin{tabular}{|c|c|c|c|c|c|c|c|c|c|}
\hline A & ABUND. & & SE & ATION & ENERGIES & (MEV) & & & \\
\hline 102 & 0.96 & $\begin{array}{r}G, N \\
10.4\end{array}$ & $\begin{array}{l}G, P \\
7.8\end{array}$ & $\begin{array}{r}G, T \\
17.4\end{array}$ & $\begin{array}{l}\text { G, HE3 } \\
15.2\end{array}$ & $\begin{array}{l}G, A \\
2.1\end{array}$ & $\begin{array}{l}G, 2 N \\
19 .\end{array}$ & $\begin{array}{l}G, N P \\
17.7\end{array}$ & $\begin{array}{l}G, 2 P \\
13.3\end{array}$ \\
\hline 104 & 10.97 & 10.0 & 8.7 & 17.0 & 16.4 & 2.6 & 17.6 & 18.0 & 14.9 \\
\hline 105 & 22.23 & 7.1 & 8.8 & 16.6 & 14.2 & 2.9 & 17.1 & 15.8 & 15.7 \\
\hline 106 & 27.33 & 9.5 & 9.3 & 16.8 & 17.6 & 3.2 & 16.6 & 18.4 & 16.4 \\
\hline 108 & 26.71 & 9.2 & 10.0 & 16.6 & 18.5 & 3.9 & 15.8 & 18.5 & 17.8 \\
\hline 110 & 11.81 & 8. & 11. & 16.4 & 19.6 & 4.4 & 15.0 & 18.7 & 19.2 \\
\hline
\end{tabular}

REF NUCLIDE REACTION RES EXCIT SOURCE DETECTOR ANG REMARKS 62MEl 46PDI05 IN,OUT NOX $\begin{array}{cccccc}2 & \text { MIN-MAX TYPE MIN-MAX } & \text { DST J-PI, TRANS PROBABL }\end{array}$ 
A ABUND.

107

109

51.35
48.65

SEPARATION ENERGIES (MEV)

$\begin{array}{lll}G, P & G, T & G, H E 3 \\ 5.8 & 13.9 & 16.4 \\ 6.5 & 13.8 & 17.3\end{array}$

$G, A$
2.8
3.3
$\begin{array}{ll}G, 2 N & G, N P \\ 17 . & 15.4 \\ 16.5 & 15.8\end{array}$

G, 2P

15. 1 16.4

\begin{tabular}{|c|c|c|c|c|c|c|c|c|c|c|c|}
\hline REF & NUCL IDE & REACTION & RES & \multirow{2}{*}{\multicolumn{2}{|c|}{ EXCIT }} & \multirow{2}{*}{\multicolumn{2}{|c|}{$\begin{array}{l}\text { SOURCE } \\
\text { MIN-MAX }\end{array}$}} & \multicolumn{2}{|c|}{ DETECTOR } & ANG & REMARKS \\
\hline & $Z \quad A$ & IN,OUT & & & & & & TYPE $N$ & $I N-M A X$ & & \\
\hline $58 M O 2$ & $47 A G$ & $G, M U-T$ & $A B X$ & THR & 94 & $C$ & 94 & $S C I-D$ & & 4P I & $*$ \\
\hline 6ORE 1 & $47 A G$ & $G, G$ & $A B X$ & 7 & & 0 & 7 & $N A I-D$ & & DST & G-WDTH \\
\hline 58301 & $47 A G$ & $G, G /$ & $A B X$ & $12-$ & -28 & $c$ & $12-28$ & & & & $*$ \\
\hline $63 K A 2$ & 47.AG & $G, G /$ & RLY & 1 & & $C$ & 5 & $A C T-1$ & & $4 \mathrm{PI}$ & \\
\hline 63VE2 & $47 A G$ & $G, G /$ & $A B X$ & 0 & 1 & $D$ & $0-1$ & $N A I-D$ & & & I SOMERS \\
\hline $57 F E 1$ & $47 A G$ & $G, N$ & RLY & 17 & 31 & $c$ & $15-31$ & THR - I & $5-$ & DST & \\
\hline $61 T A 1$ & $47 A G$ & $G, N$ & NOX & 7 & 22 & $C$ & 22 & THR - I & $6-$ & DST & \\
\hline $56 \mathrm{HA} 1$ & $47 A G$ & $G, X N$ & $A B X$ & 15, & 18 & $D$ & 15,18 & $B F 3-I$ & & $4 \mathrm{PI}$ & \\
\hline $61 B A 2$ & $47 A G$ & $G, \times N$ & $A B Y$ & $T H R$ & 22 & $C$ & 22 & THR - I & $5-$ & DST & \\
\hline $57 \mathrm{RO} 1$ & $47 A G$ & $G, P$ & RLX & 15 & 18 & C & 15,18 & & & & * REL TO CU63(G,N) \\
\hline $59 L O I$ & $47 A G$ & $G, P$ & $A B X$ & 17 & 31 & C & $16-32$ & $S C I-D$ & $4-25$ & DST & SPECTRUM \\
\hline $625 E 2$ & $47 A G$ & $G, P$ & $A B X$ & 15, & 18 & $D$ & 15,18 & $S C I-I$ & & 0 & \\
\hline 63 MI 5 & $47 A G$ & $G, X P$ & $A B Y$ & 4 & 22 & $C$ & 22 & $S C I-I$ & $4-$ & DST & \\
\hline $58 T 02$ & $47 A G$ & $G, A$ & $A B Y$ & 2 & 22 & $c$ & 22 & EMU-I & & DST & \\
\hline $63 B 13$ & $47 A G 107$ & $G, G$ & $A B X$ & 1 & & $D$ & 1 & $A C T-I$ & & $4 P I$ & MOSSBAUER \\
\hline $63 \mathrm{BO} 5$ & $47 A G 107$ & $G, G /$ & $A B X$ & 6 & 26 & C & $6-26$ & $A C T-1$ & & $4 P_{1}$ & SEPARATED ISOTOPES \\
\hline $58 B E 1$ & $47 \mathrm{AG} 107$ & $G, N$ & RLY & 9 & 10 & $C$ & $9-10$ & $A C T-I$ & & $4 P_{1}$ & THRESHOLD \\
\hline 59MU2 & $47 \mathrm{AG} 107$ & $G, N$ & $A B X$ & 9 & 21 & C & $9-25$ & $A \subset T-I$ & & $4 P I$ & \\
\hline $60 G E 3$ & $47 A G 107$ & $G, N$ & NOX & THR & & CT & HR & $B F 3-I$ & & $4 P$ I & THRESHOLD \\
\hline 61 WE 1 & $47 \mathrm{AG} 107$ & $G, N$ & $A B X$ & 9 & 11 & D & $9-11$ & $A C T-I$ & & 4P I & THRESHOLD \\
\hline 62801 & $47 \mathrm{AG} 107$ & $G, N$ & $A B X$ & 10 & 22 & $C$ & $10-22$ & $B F 3-I$ & & $4 P_{1}$ & \\
\hline $62 C A 1$ & $47 \mathrm{AG} 107$ & $G, N$ & NOX & 9 & 30 & $c$ & 30 & $A C T-1$ & & $4 \mathrm{P} \mathrm{I}$ & I SOMERIC RATIO \\
\hline $62 F \cup 6$ & $47 A G 107$ & $G, N$ & RLY & 9 & 34 & $C$ & 34 & $A C T-1$ & & $4 \mathrm{PI}$ & YLD REL TO CU62 \\
\hline 56 WA 1 & $47 A G 107$ & $G, \times N$ & $R L Y$ & & -31 & $C$ & 31 & $A C T-I$ & & $4 \mathrm{PI}$ & * REL TO TRITONS \\
\hline $56 \mathrm{WAI}$ & $47 A G 107$ & $G, T$ & RLY & $13-$ & -31 & C & 31 & $A C T-I$ & & 4P I & * REL TO NEUTRONS \\
\hline $58 \mathrm{BE} 1$ & $47 A G 109$ & $G, N$ & RLY & 9 & 10 & C & $9-10$ & $A C T-I$ & & $4 \mathrm{PI}$ & THRESHOLD \\
\hline 59NAI & $47 A G 109$ & $G, N$ & $A B X$ & 18 & & $D$ & 18 & $E F 3-1$ & & 4PI & \\
\hline $60 \mathrm{GE} 3$ & $47 A G 109$ & $G, N$ & NOX & THR & & & HR & $B F 3-1$ & & $4 P I$ & THRESHOLD \\
\hline 56 WA 1 & $47 A G 109$ & $G, \times N$ & RLY & & -31 & C. & 31 & $A C T-I$ & & 4PI & * REL TO TRITONS \\
\hline 56WAI & $47 A G 109$ & $G, T$ & RLY & $13-$ & -31 & $C^{\circ}$ & 31 & $A C T-1$ & & 4PI & * REL TO NEUTRONS \\
\hline 55ER I & $47 \mathrm{AG} 109$ & $G, A$ & $A B X$ & & -32 & C & 32 & $A C T-I$ & & $4 P I$ & * AGCL EMULSION \\
\hline $555 C 2$ & $47 A G 109$ & $G, A$ & $A B X$ & & -32 & $C$ & 32 & $A C T-I$ & & 4P I & * \\
\hline $57 E R I$ & $47 A G 109$ & $G, A$ & $A B I$ & THR & 32 & $C$ & 32 & $A C T-I$ & & 4PI & \\
\hline $59 \mathrm{RO} 3$ & $47 A G 109$ & $G, A$ & $A B X$ & & & & & $A C T-I$ & & $4 \mathrm{PI}$ & * \\
\hline
\end{tabular}




\begin{tabular}{|c|c|c|c|c|c|c|c|c|c|}
\hline A & ABUND. & $G, N$ & $\begin{array}{l}S E \\
G, P\end{array}$ & $\begin{array}{l}A T \text { I ON } \\
\text { G, T }\end{array}$ & $\begin{array}{r}\text { ENERGIES } \\
\text { G, HE } 3\end{array}$ & $\begin{array}{l}\text { (MEV) } \\
\text { G, A }\end{array}$ & $\mathrm{G}, 2 \mathrm{~N}$ & $G$, NP & $G, 2 P$ \\
\hline 106 & 1.22 & 11. & 7. & 17.2 & 14.6 & 1.6 & 19. & 17.4 & $12 \cdot 3$ \\
\hline 108 & 0.88 & $10 \cdot 3$ & 8.1 & 17. & 15.7 & $2 \cdot 3$ & $18 \cdot 3$ & 17.7 & 13.9 \\
\hline 110 & $12 \cdot 39$ & 9.9 & 8.9 & 16.9 & 16.9 & 2.9 & 17.2 & 18.1 & 15.4 \\
\hline 111 & 12.75 & 7.0 & 9.1 & 16.6 & 14.6 & 3.3 & 16.8 & 15.7 & 16.2 \\
\hline 112 & 24.07 & 9.4 & 9.7 & 16.8 & 17.9 & 3.5 & 16.4 & 18.5 & 16.8 \\
\hline 113 & 12.26 & 6.5 & 9.7 & 16.5 & 15.6 & 3.9 & 15.9 & 16.2 & 17.6 \\
\hline 114 & 28.86 & 9.0 & 10.3 & 16.8 & 18.9 & 4.1 & 15.6 & 18.8 & 18.3 \\
\hline 116 & 7.58 & 8.7 & 11.2 & * & 16.6 & 4.9 & 14.7 & 18.7 & * \\
\hline
\end{tabular}

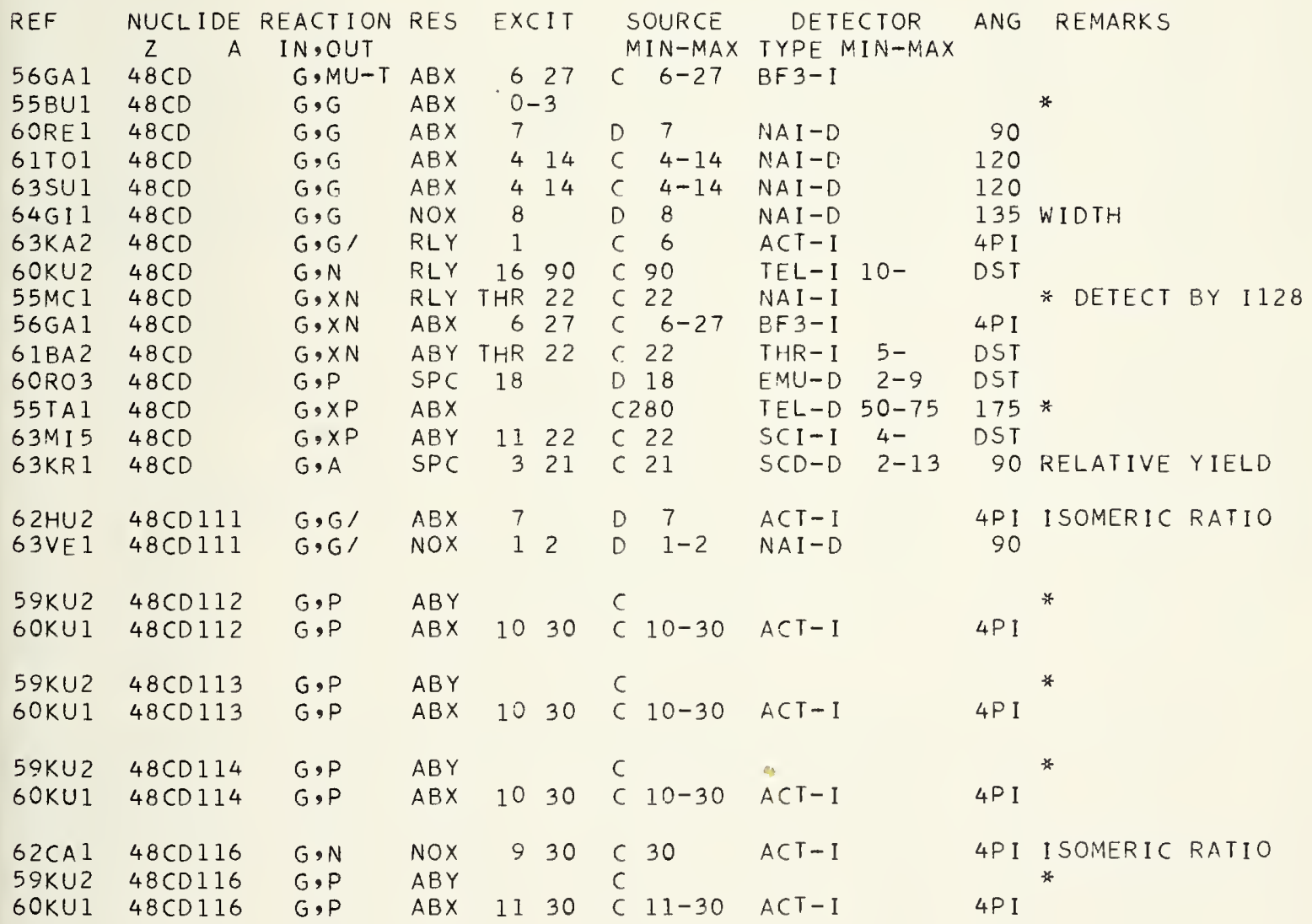


A ABUND.

113

115

REF

$55 B \cup 1$

$55 B \cup 2$

$63 K A 2$

$56 \mathrm{HE} 2$

$57 \mathrm{RO} 2$

6OBA 5

$58 B A 4$

$60 \mathrm{CHI}$

$63 \mathrm{MI} 5$

$60 \mathrm{CHI}$

$60 \mathrm{CHI}$

$56 \mathrm{HE} 2$

$58 \mathrm{TO} 2$

$62 \mathrm{CA}$

56BO 1

$62 \mathrm{HU} 2$

63 VE 1

56BOI

$58 B E 1$

60 GE 3

$62 \mathrm{AN} 1$

62BO 1

4.28
95.72

$$
\begin{aligned}
& G, N \\
& 9.4 \\
& 9.0
\end{aligned}
$$

SEPARATION ENERGIES (MEV)

$\begin{array}{llll}G, P & G, T & G, H E 3 & G, A \\ 6.1 & 13.9 & 16.8 & 3.0 \\ 6.8 & 13.9 & 17.9 & 3.8\end{array}$

\begin{abstract}
$\mathrm{G}, 2 \mathrm{~N}$
17.3

16.3
\end{abstract}

G, 2P

15.7 17.1

\begin{tabular}{|c|c|c|c|c|c|c|c|c|c|c|}
\hline NUCL IDE & REACTION & RES & $E \times C$ & IT & & SOURCE & DETE & CTOR & ANG & REMARKS \\
\hline $\begin{array}{l}Z \\
49 \mathrm{IN}\end{array}$ & $\begin{array}{l}\text { IN, OUT } \\
G, G\end{array}$ & $A B X$ & $0-$ & & & IIN-MAX & TYPE N & IIN-MAX & & \\
\hline $49 \mathrm{IN}$ & $G, G /$ & $A B X$ & 0 & 14 & $c$ & $1-14$ & $A C T-I$ & & 4PI & \\
\hline 49 IN & $G, G /$ & RLY & 1 & & $c$ & 6 & $A C T-I$ & & $4 P I$ & \\
\hline $49 \mathrm{IN}$ & $G, N$ & RLY & THR- & -31 & $\mathrm{C}$ & 31 & & & & * REL TO ALPHAS \\
\hline $49 I N$ & $G, P$ & SPC & & & D & & & & DST & * \\
\hline $49 \mathrm{IN}$ & $G, P$ & $A B X$ & 16 & 40 & C & $16-40$ & $M A G-D$ & & DST & \\
\hline 49 IN & $G, X P$ & RLX & THR & 40 & $c$ & -40 & $M A G-D$ & & DST & * SPECTRUM \\
\hline $49 \mathrm{IN}$ & $G, \times P$ & RLX & THR & 90 & $c$ & 90 & TEL-I & $15-30$ & 90 & REL TO DEUTERONS \\
\hline $49 \mathrm{IN}$ & $G, \times P$ & $A B Y$ & 8 & 22 & $c$ & 22 & $S C I-I$ & 4- & DST & \\
\hline $49 \mathrm{IN}$ & $G, \times D$ & $R L X$ & THR & 90 & $\mathrm{C}$ & 90 & TEL-I & $15-30$ & 90 & REL TO PROTONS \\
\hline $49 \mathrm{IN}$ & $G, \times T$ & RLY & THR & 90 & C & 90 & TEL-I & $15-30$ & 90 & REL TO DEUTERONS \\
\hline $49 I N$ & $G, A$ & RLY & THR- & -31 & $\mathrm{C}$ & 31 & & & & * REL TO NEUTRONS \\
\hline $49 \mathrm{IN}$ & $G, A$ & $A B Y$ & 1 & 22 & $c$ & 22 & EMU-I & & DST & \\
\hline 49 IN113 & $G, N$ & NOX & 6 & 30 & $c$ & 30 & $A C T-I$ & & $4 P I$ & ISOMERIC RATIO \\
\hline 49 IN 115 & $G, G /$ & $A B X$ & 5 & 27 & C & $5-27$ & $A C T-I$ & & $4 \mathrm{PI}$ & \\
\hline 49 IN 115 & $G, G /$ & NOX & 7 & & D & 7 & $A C T-I$ & & $4 P I$ & I SOMER IC RATIO \\
\hline 49 IN 115 & $G, G /$ & NOX & 1 & 2 & D & $1-2$ & NAI -D & & 90 & \\
\hline 49 IN 115 & $G, N$ & $A B X$ & 9 & 27 & $c$ & $9-27$ & BF 3-I & & 4P I & \\
\hline $49 I N 115$ & $G, N$ & RLY & 9 & 10 & C & $9-10$ & $A C T-I$ & & 4PI & THRESHOLD \\
\hline $491 N 115$ & $G, N$ & NOX & THR & & & $H R$ & $B F 3-1$ & & $4 P I$ & THRESHCLD \\
\hline 49 IN 115 & $G, N$ & SPC & 9 & 28 & C & 28 & EMU-D & $1-11$ & DST & \\
\hline 49INII5 & $G, N$ & $A B X$ & 10 & 23 & C & $10-23$ & $B F 3-I$ & & $4 \mathrm{PI}$ & \\
\hline
\end{tabular}

TIN $Z=50$

\begin{tabular}{|c|c|c|c|c|c|c|c|c|c|}
\hline A & ABUND. & & SEF & ATION & ENERGIES & (MEV) & & & \\
\hline 112 & 0.96 & $G, N$ & G, $P$ & G, T & $\mathrm{G}, \mathrm{HE} 3$ & $\mathrm{G}, \mathrm{A}$ & $G, 2 N$ & G,NP & $G, 2 P$ \\
\hline 114 & 0.66 & 10.3 & 8.5 & 14.3 & 15.3 & 2.6 & 18.1 & 18.0 & 14.6 \\
\hline 115 & 0.35 & 7.5 & 8.7 & 17.0 & 14.4 & 3.2 & 17.9 & 16.1 & 15.6 \\
\hline 116 & 14.30 & 9.6 & 9.3 & 17.1 & 17.4 & 3.4 & 17.1 & 18.3 & 16.1 \\
\hline 117 & 7.61 & 6.9 & 9.5 & 16.8 & 15.3 & 3.8 & 16.5 & 16.2 & 16.9 \\
\hline 118 & 24.03 & 9.3 & 10.0 & 17.1 & 18.5 & 4.1 & 16.3 & 18.8 & 17.5 \\
\hline 119 & 8.58 & 6.5 & 9.9 & 16.8 & 16.3 & 4.4 & 15.8 & 16.5 & 18.2 \\
\hline 120 & 32.85 & 9.1 & 10.8 & 17.1 & 19.6 & 4.8 & 15.6 & 19.0 & 19.0 \\
\hline 122 & 4.72 & 8.8 & 11.6 & 17.3 & 20.8 & 5.7 & 15.0 & 20. & * \\
\hline 124 & 5.94 & 8.5 & 12. & 18. & * & * & 14.4 & 20.4 & * \\
\hline
\end{tabular}

REF

56FU1 6ORE 1

GITO1

62BE 2

$63 \mathrm{AX} 1$

$63 \mathrm{KA} 3$

64AR 1

63KA2
NUCL IDE REACTIION RES $Z$ A IN,OUT

$50 S \mathrm{~N}$

$50 S N$

$50 S N$

$5 O S N$

$50 S N$

$50 S N$

$505 N$

5 OSN

$G, G$
$G, G$
$G, G$
$G, G$
$G, G$
$G, G$
$G, G$
$G, G /$

$A B X$

$A B X$

$A B X$

$A B X$

$A B X$

$A B X$

$A B X$

RLY
EXCI

$\begin{array}{ll}4 & 40 \\ 7 & \\ 3 & 15 \\ 5 & 9 \\ 6 & 10 \\ 1,1 \\ 6,7 \\ 1\end{array}$

SOURCE

MIN-MAX

C $4-40$

D 7

C 3-15

D $5-9$

D $6-10$

D 1,1

D 6,7

C 6
DETECTOR ANG REMARKS

NAI -D

NAI - D

NAI -D

NAI -D

NAI -D

NAI - D

NAI -D

ACT-I
120

DST G-WDTH

120

135

135

120

135

4P I 


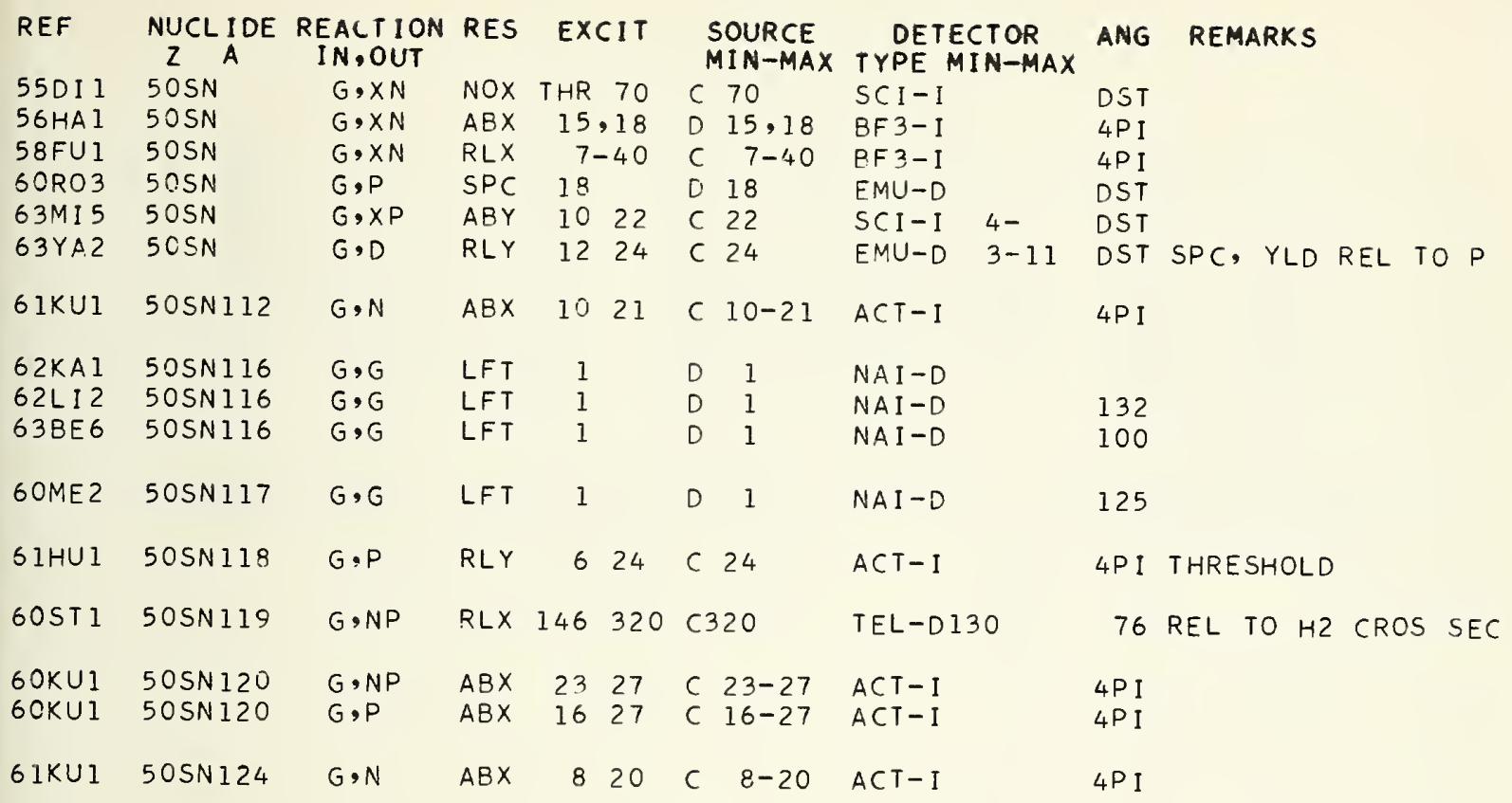

ANT IMONY $Z=51$

\begin{tabular}{|c|c|c|c|c|c|c|c|c|c|}
\hline A & ABUND. & & SE & AT ION & ENERGIES & (MEV) & & & \\
\hline $\begin{array}{l}21 \\
23\end{array}$ & $\begin{array}{l}57.25 \\
42.75\end{array}$ & $\begin{array}{l}G, N \\
9.3 \\
9.0\end{array}$ & $\begin{array}{l}G, P \\
5.8 \\
6.6\end{array}$ & $\begin{array}{r}G, T \\
12.9 \\
13.1\end{array}$ & $\begin{array}{l}\text { G, HE } 3 \\
17.1 \\
18 .\end{array}$ & $\begin{array}{l}G, A \\
3.1 \\
4.1\end{array}$ & $\begin{array}{l}G, 2 N \\
16.3 \\
15.8\end{array}$ & $\begin{array}{l}G, N P \\
14.9 \\
15.4\end{array}$ & $\begin{array}{l}G, 2 P \\
16.6 \\
18 .\end{array}$ \\
\hline
\end{tabular}

\begin{tabular}{|c|c|c|c|c|c|c|c|c|c|}
\hline REF & NUCL IDE & REACTION & RES & EXCIT & SOURCE & DETE & ETOR & ANG & REMARKS \\
\hline & ${ }^{2} 158$ & IN, OUT & & & MIN-MAX & TYPE N & IN-MAX & & \\
\hline $\begin{array}{l}\text { 60RE1 } \\
\text { 63SU1 }\end{array}$ & $\begin{array}{l}51 S B \\
51 S E\end{array}$ & $\begin{array}{l}G, G \\
G, G\end{array}$ & $\begin{array}{l}A B X \\
A B X\end{array}$ & $\begin{array}{ll}7 & \\
4 & 14\end{array}$ & $\begin{array}{ll}\text { D } & 7 \\
\text { C } & 4-14\end{array}$ & $\begin{array}{l}\text { NAI I-D } \\
\text { NAI - D }\end{array}$ & & $\begin{array}{r}90 \\
120\end{array}$ & \\
\hline $56 \mathrm{HE} 2$ & $51 \mathrm{SB}$ & $G, N$ & RLY & $T H R-31$ & C 31 & & & & * REL TO ALPHAS \\
\hline 63YA2 & $515 B$ & $G, D$ & RLY & 1024 & C 24 & $E M U-D$ & $3-11$ & 126 & $S P C, Y L D$ REL TO $P$ \\
\hline $56 \mathrm{HE} 2$ & $515 B$ & $G, A$ & RLY & $T H R-31$ & C 31 & & & & * REL TO NEUTRONS \\
\hline 63ME 1 & $51 S B 121$ & $G, G$ & LFT & 1 & D & NAI - D & & DST & $J-P I$ \\
\hline $60 \mathrm{GE} 3$ & $515 B 121$ & $G, N$ & NOX & THR & CTHR & BF 3-I & & $4 \mathrm{PI}$ & THRESHOLD \\
\hline $61 \mathrm{CO} 2$ & $51 S B 121$ & $G, N$ & $R L X$ & 15,18 & D 15,18 & $A C T-I$ & & $4 \mathrm{PI}$ & REL TO CU63 SIGM \\
\hline $62 D$ & $515 B 121$ & $G, N$ & $A B X$ & 20 & D 20 & $A C T-I$ & & $4 \mathrm{PI}$ & \\
\hline $57 E R 1$ & $515 B 121$ & $G, D$ & $A B X$ & $18 \quad 32$ & C $18-32$ & $A C T-I$ & & 4P I & \\
\hline $55 E$ & $51 S B 121$ & $G, A$ & $A B X$ & $3-32$ & C 32 & $A C T-I$ & & 4PI & * \\
\hline 61 WO & $515 B 121$ & $G, A$ & $A B X$ & 1524 & C $15-24$ & $A C T-I$ & & $4 \mathrm{PI}$ & \\
\hline OG & $515 B 1$ & $G$, & NOX & THR & CTHR & BF 3-I & & 4P I & THRESHOLD \\
\hline 0 & $515 B 123$ & $G, N P$ & RLY & $18 \quad 32$ & C 32 & $A C T-I$ & & $4 \mathrm{PI}$ & THRESHOLD \\
\hline
\end{tabular}




\begin{tabular}{|c|c|c|c|c|c|c|c|c|c|}
\hline A & ABUND. & & $\mathrm{SE}$ & ATION & ENERGIES & (MEV) & & & \\
\hline & & $G, N$ & $G, P$ & $G, T$ & $\mathrm{G}, \mathrm{HE} 3$ & $G, A$ & $G, 2 N$ & $G, N P$ & $G, 2 P$ \\
\hline 120 & $8 \cdot 9(-2)$ & 10.3 & 7.2 & 15.8 & 13.9 & 0.3 & 18. & 16.8 & $12 \cdot 3$ \\
\hline 122 & 2.46 & 10.1 & 8.0 & 15.8 & 15.2 & 1.1 & 17.0 & 17.3 & 13. \\
\hline 123 & 0.87 & 6.9 & 8.1 & 15.7 & 13.0 & 1.5 & 17.0 & 15.0 & 14. \\
\hline 124 & 4.61 & 9.4 & 8.6 & 15.9 & 16.2 & 1.8 & 16.4 & 17.6 & 5. \\
\hline 125 & 6.99 & 6.6 & 8.7 & 15.7 & 14.0 & $2 \cdot 2$ & 16.0 & 15.2 & 5. \\
\hline 126 & 18.71 & 9.1 & 9.1 & 15.8 & 17.2 & 2.5 & 15.7 & 17.9 & 16. \\
\hline 128 & 31.79 & 8.8 & 9.6 & 15.7 & 18.0 & 3.2 & 15.1 & 18.0 & 17.5 \\
\hline 130 & 34.48 & 8.4 & 10.1 & 15.6 & 18.7 & 3.7 & 14.5 & 18.0 & 18. \\
\hline
\end{tabular}

\begin{tabular}{|c|c|c|c|c|c|c|c|c|c|c|}
\hline REF & NUCL IDE & REACT ION & RES & EXCIT & $\begin{array}{l}\text { SOURCE } \\
\text { MIN-MAX }\end{array}$ & DET & CTOR & ANG & REMARKS & \\
\hline & $\begin{array}{ll}Z & A\end{array}$ & IN,OUT & & & MIN-MAX & TYPE 1 & IN-MAX & & & \\
\hline 6ORE 1 & $52 T E$ & $G, G$ & $A B X$ & 7 & D 1 & $N A I-D$ & & DST & & \\
\hline 64PA1 & $52 T E$ & $G, G$ & LFT & 1 & D & NAI I D & & 90 & & \\
\hline $60 \mathrm{HAl}$ & $52 \mathrm{TE}$ & $G, A$ & SPC & 833 & C 33 & $E M U-D$ & $8-14$ & DST & & \\
\hline $63 \mathrm{SH} 5$ & 52TE 122 & $G, G$ & LFT & 1 & D & NAI I D & & 105 & & \\
\hline $61 \mathrm{AK} 1$ & 52TE124 & $G, G$ & LFT & 1 & D & & & & & \\
\hline 60GE3 & 52 TE 125 & $G, N$ & NOX & THR & CTHR & $8 F 3-I$ & & $4 \mathrm{PI}$ & THRESHOLD & \\
\hline 6OGE 3 & 52 TE 126 & $G, N$ & NOX & THR & CTHR & $B F 3-I$ & & 4PI & THRESHOLD & \\
\hline 60GE3 & 52 TE 128 & $G, N$ & NOX & THR & CTHR & BF $3-I$ & & 4PI & THRESHOLD, ALSO & 30 \\
\hline OGE3 & 52 TEI30 & $G, N$ & NOX & THR & CTHR & $B F 3-I$ & & $4 P I$ & THRESHOLD, ALSO & 28 \\
\hline
\end{tabular}

I ODINE $Z=53$

\begin{tabular}{|c|c|c|c|c|c|c|c|c|}
\hline A & ABUND. & & SE & ATION & ENERGIES & (MEV) & & \\
\hline & & $G, N$ & $G, P$ & $G, T$ & G, HE3 & $G, A$ & $G, 2 N$ & $G, N P$ \\
\hline 27 & 0 & 9.1 & & & 16.3 & & 16.2 & 15.4 \\
\hline
\end{tabular}

\begin{tabular}{|c|c|c|c|c|c|c|c|c|c|c|c|c|}
\hline$E F$ & $\mathrm{NUCl}$ & IDE & REACTION & RES & EX & CIT & & OURCE & DET & ECTOR & ANG & REMARKS \\
\hline & $z$ & A & IN,OUT & & & & & IN-MAX & TYPE & MIN-MAX & & \\
\hline $56 G A 1$ & $53 I$ & $\begin{array}{l}127 \\
127\end{array}$ & G,MU-T & $\begin{array}{l}A B X \\
A B X\end{array}$ & $\begin{array}{l}9 \\
4\end{array}$ & $\begin{array}{l}27 \\
40\end{array}$ & c & $\begin{array}{l}9-27 \\
4-40\end{array}$ & $B F 3-I$ & & & \\
\hline & $53 \mathrm{I}$ & 127 & $G, G$ & $A B X$ & 4 & 40 & c & $4-40$ & $N A I-D$ & & 120 & \\
\hline 6ORE 1 & 53 I & 127 & $G, G$ & $A B X$ & 7 & & D & 7 & $N A I-D$ & & 90 & \\
\hline $56 E R 1$ & $53 I$ & 127 & $G, N$ & $A B X$ & 15 &, 18 & D & 15,18 & $A C T-I$ & & 4PI & \\
\hline $57 F E 2$ & $53 \mathrm{I}$ & 127 & $G, N$ & RLY & 14 & 30 & $c$ & $14-30$ & THR - I & $5-14$ & 90 & \\
\hline $58 \mathrm{CH} 2$ & $53 I$ & 127 & $G, N$ & RLY & THR & & & $H R$ & $B F 3-I$ & & 4PI & THRESHOLD \\
\hline $60 G E 3$ & $53 I$ & 127 & $G, N$ & NOX & THR & & & $H R$ & $B F 3-I$ & & 4PI & THRESHOLD \\
\hline OKU2 & 53 I & 127 & $G, N$ & RLY & 16 & 90 & $c$ & 90 & TEL-I & $10-$ & DST & \\
\hline 6OREI & $53 I$ & 127 & $G, N$ & $A B X$ & 7 & & D & 7 & NAI I I & & 90 & \\
\hline 6INAI & 53 I & 127 & $G, N$ & $A B X$ & 8 & 22 & C & $10-20$ & $A C T-I$ & & $4 P I$ & \\
\hline $61 \mathrm{TAl}$ & $53 \mathrm{I}$ & 127 & $G, N$ & NOX & 12 & 22 & $c$ & 22 & THR - I & $6-$ & DST & \\
\hline $56 E R 1$ & $53 I$ & 127 & $G, 2 N$ & $\mathrm{ABX}$ & 15 & 18 & D & 15,18 & $A C T-I$ & & $4 P I$ & \\
\hline 6INAI & $53 I$ & 127 & $G, 2 N$ & $A B X$ & 16 & 22 & $c$ & $10-20$ & $B F 3-I$ & & $4 \mathrm{PI}$ & ALSO ACTIVATION \\
\hline $58 \mathrm{FUI}$ & 53 I & 127 & $G, \times N$ & RLX & & -40 & $c$ & $7-40$ & $B F 3-I$ & & 4PI & \\
\hline $56 G A 1$ & 53 I & 127 & $G, \times N$ & $A B X$ & 9 & 27 & $c$ & $9-27$ & $B F 3-I$ & & $4 \mathrm{PI}$ & \\
\hline $58 \mathrm{KAl}$ & 53 I & 127 & $G, \times N$ & $A B X$ & 9 & 22 & $c$ & $9-22$ & $B F 3-I$ & & $4 \mathrm{PI}$ & \\
\hline $61 \mathrm{BA} 2$ & $53 \mathrm{I}$ & 127 & $G, \times N$ & $A B Y$ & THR & 22 & $c$ & 22 & THR-I & $5-$ & DST & \\
\hline $58 \mathrm{KE} 1$ & $53 I$ & 127 & $G, P$ & $A B X$ & 18 & & D & 18 & $S C I-D$ & $6-11$ & 0 & SPECTRUM \\
\hline & $53 I$ & 127 & $G, P$ & $A B X$ & 15 & 18 & D & 15,18 & $S C I-D$ & & & * SPC, CSI TARGET \\
\hline OTAI & $53 I$ & 127 & $G, P$ & $A B X$ & 14 & 32 & $c$ & $14-32$ & $S C I-D$ & $3-24$ & 4PI & SPC, CSI TARGET \\
\hline ISE 4 & $53 I$ & 127 & $G, P$ & $A B X$ & 15 & 18 & D & 15,18 & $S C I-D$ & & 4P I & CSI TARGET, SPC \\
\hline
\end{tabular}




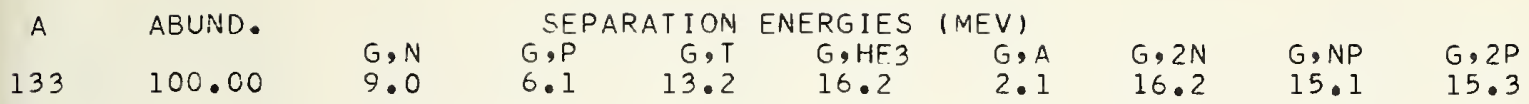

\begin{tabular}{|c|c|c|c|c|c|c|c|c|}
\hline REF & $\begin{array}{r}\text { NUCL IDE } \\
Z\end{array}$ & $\begin{array}{l}\text { REACT ION } \\
\text { IN, OUT }\end{array}$ & RES & EXCIT & $\begin{array}{l}\text { SOURCE } \\
\text { MIN-MAX }\end{array}$ & $\begin{array}{c}\text { DETECTOR } \\
\text { TYPE MIN-MAX }\end{array}$ & ANG & REMARKS \\
\hline $58 \mathrm{CH} 2$ & $55 C S 133$ & $G, N$ & RLY & THR & CTHR & $B F 3-I$ & 4P I & THRESHOLD \\
\hline $60 \mathrm{GE} 3$ & $55 C S 133$ & $G, N$ & NOX & THR & CTHR & BF3-I & 4P I & THRESHOLD \\
\hline $58 \mathrm{KAl}$ & $55 C S 133$ & $G, \times N$ & $A B X$ & 922 & C $9-22$ & BF 3-I & 4P I & \\
\hline 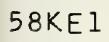 & $55 C S 133$ & $G, P$ & $A B X$ & 18 & D 18 & $S C I-D$ & 0 & SPECTRUM \\
\hline 1 & $55 \operatorname{CS} 133$ & $G, P$ & $A B X$ & 15,18 & D 15,18 & $S C I-D$ & & * SPC, CSI TARGET \\
\hline$A 1$ & $55 C S 133$ & $G, P$ & $A B X$ & $14 \quad 32$ & C $14-32$ & $S C I-D$ & $4 \mathrm{PI}$ & SPC, CSI \\
\hline SE4 & $55 C 5133$ & $G, P$ & $A B X$ & 15,18 & D 15,18 & $S C I-D$ & $4 P I$ & CSI TARGET, SPC \\
\hline
\end{tabular}

BARIUM $Z=56$

\begin{tabular}{|c|c|c|c|c|c|c|c|c|c|}
\hline \multirow[t]{2}{*}{ A } & \multirow{2}{*}{ ABUND. } & & \multicolumn{2}{|c|}{ SEPARATION } & ENERGIES & \multicolumn{2}{|l|}{ (MEV) } & \multirow[b]{2}{*}{$G, N P$} & \multirow[b]{2}{*}{$G, 2 P$} \\
\hline & & $G, N$ & $G, P$ & $G, T$ & G, HE 3 & $G, A$ & $\mathrm{G}, 2 \mathrm{~N}$ & & \\
\hline $\begin{array}{l}130 \\
132\end{array}$ & $\begin{array}{r}0.10 \\
0 \quad 71-2\end{array}$ & 10. & $7 \cdot$ & 16.1 & 14.0 & 0.6 & 18 & 16.8 & 12.0 \\
\hline 134 & 2.42 & 9.2 & 8.0 & 15.7 & 15.4 & 1.4 & 16.6 & 17.1 & 14.2 \\
\hline 135 & 6.59 & 7.2 & 8.5 & 15.7 & 13.6 & 2.0 & 16.4 & 15.2 & 14.8 \\
\hline 136 & 7.81 & 9.2 & 8.7 & 15.9 & 16.3 & 2.3 & 16.4 & 17.7 & 15.6 \\
\hline 37 & $11 \cdot 32$ & 6.9 & 9.0 & 16.2 & 14.8 & 2.7 & 16.2 & 15.6 & 16.0 \\
\hline 38 & 71.66 & 8 & 8.9 & 15.7 & 16.8 & 2.8 & 15.5 & 17.6 & 16.6 \\
\hline
\end{tabular}

REF

NUCLIDE REACTION RES EXCIT

SOURCE

DETECTOR

ANG REMARKS

$Z$ A IN,OUT

MIN-MAX TYPE MIN-MAX

6

$$
6
$$

$G, G$

$G, G /$

ABX 7

D 7

NAI I D

G,N RLY 1731

C 5

$A C T-I$

THR-I

$G, X N$ ABY THR 22

C 15-31

THR-I 5-

EMU

90
4PI
DST SPECTRUM
DST
$*$

$G, A$

CTHR

BF 3-I

4P I THRESHOLD 
$\mathrm{Ce}$

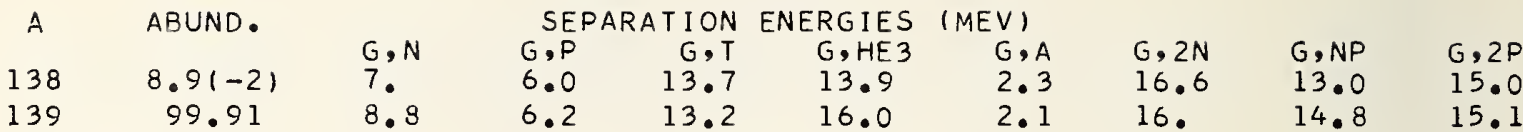

\begin{tabular}{|c|c|c|c|c|c|c|c|c|c|c|}
\hline REF & $\underset{Z}{N U C L}$ IDE & $\begin{array}{l}\text { REACT ION } \\
\text { IN, OUT }\end{array}$ & RES & EXCIT & & $\begin{array}{l}\text { OURCE } \\
\text { IN-MAX }\end{array}$ & $\begin{array}{c}D E T \\
\text { TYPE }\end{array}$ & $\begin{array}{l}\text { CTOR } \\
\text { IN-MAX }\end{array}$ & ANG & REMARKS \\
\hline $58 \mathrm{SP} 1$ & $57 \mathrm{LA}$ & $G, N$ & $\operatorname{RL} x$ & 818 & $c$ & $8-18$ & $B F 3-I$ & & $4 P I$ & \\
\hline ITAl & 57LA & $G, N$ & NOX & 1122 & $c$ & 22 & THR-I & $6-$ & DST & - \\
\hline $2 M I 3$ & $57 L A$ & $G, N$ & $A B X$ & $10 \quad 21$ & D & $10-21$ & $B F 3-I$ & & $4 \mathrm{PI}$ & \\
\hline 2RE 1 & $57 \mathrm{LA}$ & $G, N$ & NOX & 1155 & $c$ & 55 & THR-I & $6-$ & DST & \\
\hline YUI & $57 \mathrm{LA}$ & $G, \times N$ & $A B Y$ & $7-40$ & c & $7-40$ & $B F 3-I$ & & $4 P I$ & \\
\hline BA 2 & $57 L A$ & $G, \times N$ & $A B Y$ & THR 22 & $c$ & 22 & THR - I & $5-$ & DST & \\
\hline 1 MI 1 & $57 L A$ & $G, X N$ & $A B X$ & $6 \quad 22$ & D & $6-22$ & $B F 3-I$ & & $4 P I$ & \\
\hline +RI I & $57 \mathrm{LA}$ & $G, \times N$ & $A B X$ & $8-30$ & $c$ & $8-30$ & $B F 3-I$ & & $4 P I$ & \\
\hline & $57 L A 139$ & $G, N$ & RLY & THR & & HR & $\mathrm{BF} 3-$ & & 4P I & 1OLD \\
\hline & $57 L A 139$ & $G, N$ & NOX & THR & & HR & BF 3-I & & 4P I & THRESHOLD \\
\hline $8 \mathrm{KA}$ & 57 LA 139 & $G, \times N$ & $A B X$ & 922 & C & $9-22$ & $B F 3-I$ & & 4P I & \\
\hline
\end{tabular}

\section{CERIUM $Z=58$}

\begin{tabular}{|c|c|c|c|c|c|c|c|c|c|}
\hline \multirow[t]{2}{*}{ A } & ABUND. & \multicolumn{3}{|c|}{ SEPARATION } & ENERGIES & \multicolumn{2}{|l|}{ (MEV) } & \multirow[b]{2}{*}{$G, N P$} & \\
\hline & & $G, N$ & $G, P$ & $G, T$ & G, HE 3 & $G, A$ & $G, 2 N$ & & $G, 2 P$ \\
\hline 136 & 0.19 & 10. & 7. & 16.0 & $13 \cdot 8$ & .6 & 17.7 & 16.9 & $12 \cdot 3$ \\
\hline & 0.25 & 9. & 7. & 16. & 14.7 & 1.2 & 17.3 & 15.8 & \\
\hline 140 & 88.48 & 9.0 & 8.0 & 16. & 15.0 & 1.4 & 16.6 & 16.8 & \\
\hline 42 & 11.07 & 7.2 & 8.9 & 12.1 & 14.4 & -1.4 & 12.6 & 15.7 & \\
\hline
\end{tabular}

\begin{tabular}{|c|c|c|c|c|c|c|c|c|c|c|c|c|}
\hline REF & NUCL IDE & REACTION & RES & EX & I T & & OURCE & DETE & TOR & ANG & REMARKS & \\
\hline & $2 \quad A$ & IN,OUT & & & & & IN-MAX & TYPE M & $N-\operatorname{MAX}$ & & & \\
\hline $\begin{array}{l}648 E 4 \\
62 M I 3\end{array}$ & $\begin{array}{l}58 C E \\
58 C E\end{array}$ & $\begin{array}{l}G, G \\
G, N\end{array}$ & $\begin{array}{l}\text { LFT } \\
A B X\end{array}$ & $\begin{array}{r}2 \\
10\end{array}$ & 21 & $\begin{array}{l}D \\
D\end{array}$ & ${ }^{2}-21$ & $\begin{array}{l}\text { NA I - D } \\
\text { BF 3-I }\end{array}$ & & $\begin{array}{l}120 \\
4 \mathrm{PI}\end{array}$ & & \\
\hline $58 \mathrm{FUl}$ & $58 C E$ & $G, X N$ & $A B Y$ & & -40 & $c$ & $7-40$ & $B F 3-I$ & & 4P I & & \\
\hline $61 \mathrm{BA} 2$ & $58 C E$ & $G, \times N$ & $A B Y$ & THR & 22 & $c$ & 22 & THR - I & $5-$ & DST & & \\
\hline $61 M I 1$ & $58 C E$ & $G, \times N$ & $A B X$ & 6 & 22 & D & $6-22$ & BF 3-I & & 4P I & & \\
\hline $59 \mathrm{HA2}$ & $58 C E$ & $G, A$ & & & & & & EMU & & & * & \\
\hline $\begin{array}{l}62 \text { CA } 1 \\
62 \text { FU6 }\end{array}$ & $\begin{array}{l}58 \mathrm{CE} 140 \\
58 \mathrm{CE} 140\end{array}$ & $\begin{array}{l}G, N \\
G, N\end{array}$ & $\begin{array}{l}\text { NOX } \\
\text { RLY }\end{array}$ & $\begin{array}{l}5 \\
5\end{array}$ & $\begin{array}{l}30 \\
31\end{array}$ & $\begin{array}{l}c \\
C\end{array}$ & $\begin{array}{l}30 \\
31\end{array}$ & $\begin{array}{l}A C T-I \\
A C T-I\end{array}$ & & $\begin{array}{l}4 P I \\
4 P I\end{array}$ & I SOMER IC & RATIO \\
\hline SOGE 3 & $58 C E 142$ & $G, N$ & NOX & THR & & $C T$ & $H R$ & BF 3-I & & 4PI & THRESHOLD & \\
\hline
\end{tabular}



A ABUND.
SEPARATION ENERGIES (MEV)
$141 \quad 100.00$
$G, N$
9.4
$G, P$ G,
$G, H E$
$G, A$
$G, 2 N$
$G, N P$
$G, 2 P$
13.3
$14 \cdot 3$
I. $\quad 17.1$
$14 \cdot 3$
13.2

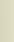

REF NUCLIDE REACTION RES $Z$ A IN,OUT

$61 B 03$ 59PR141 G,MU-T

$62 \mathrm{BE} 2$

$G, G$

59PR 141

$G, G$

$G, N$

S9PR 141

$58 \mathrm{CH} 2$

$59 C A 2$

59PR 141

$G, N$

$G, N$

$G, N$

$G, N$

$G, N$

$G, N$

$G, N$

$G, N$

$G, N$

$G, 2 N$

$G, 2 N$

$G, 2 N$

$G, 3 N$

$G, X N$

$G, X N$

$G, X N$

$G, P$

$61 \mathrm{MO} 1$

$58 \mathrm{KAl}$

62TH1

$64 R I 1$

$61 \mathrm{SH} 2$

$625 \mathrm{H}_{4}$

$59 P R 1$

$50 P R 141$

59PR 141

59PR 141

$G, P \quad S P C \quad 5 \quad 34$

SOURCE DETECTOR ANG REMARKS

MIN-MAX TYPE MIN-MAX

EXCIT

$10-20$

$10-20$

D $5-9$

D 6-9

CTHR

RLY THR

$A B X \quad 10 \quad 32$

$A B X \quad 10 \quad 32$

$A B X \quad 9 \quad 30$

NOX THR

ABX 929

$A B X \quad 15,18$

NOX 1222

$A B X \quad 20$

C 33

C $10-32$

C 30

CTHR

THR - I
NAI $-D$

NAI -D

BF 3-I

ACT-I

ACT - I

ACT-I

ACT-I

C 10-29 ACT-I

D 15,18

C 22

D 21

C 55

$A B X \quad 18 \quad 32$

c 33

C 30

C $10-29$

C $27-33$

ACT-I

THR - I

ACT-I

THR-I

ACT-I

$A C T-I$

$A B X \quad 16 \quad 29$

ABI $27 \quad 33$

$A B X \quad 10 \quad 22$

C $10-22$

ACT-I

$A C T-I$

BF 3-I

C 7-18 BF3-I

c $9-30$

BF 3-I

EMU-D

C 23,34

EMU-D

$\begin{array}{lll} & \text { 4PI } & * \\ & 135 & \\ & 135 & \\ & 4 P I & \text { THRESHOLD } \\ & 4 P I & \\ & 4 P I & \\ & 4 P I & \text { THRESHOLD } \\ & 4 P I & \text { THRESHOLD } \\ & 4 P I & \\ & 4 P I & \\ 6- & \text { DST } & \\ & 4 P I & \\ & \text { DST } & \\ & 4 P I & \text { THRESHOLD } \\ & 4 P I & \text { THRESHOLD } \\ & 4 P I & \\ & 4 P I & \text { THRESHOLD } \\ & 4 P I & \\ & 4 P I & \\ & 4 P I & \\ 4-18 & \text { DST } & \\ 4-22 & \text { DST } & \\ & & \end{array}$

NEODYMIUM $Z=60$

\begin{tabular}{|c|c|c|c|c|c|c|c|c|c|}
\hline A & ABUND. & & SEF & T ION & ENERGIES & (MEV) & & & \\
\hline & & $G, N$ & $G, P$ & $G, T$ & G, HE 3 & $G, A$ & $G, 2 \mathrm{~N}$ & $G, N P$ & $G, 2 P$ \\
\hline 142 & 27.11 & 9.8 & 7.2 & 15.8 & 13.8 & 0.7 & 17.7 & 16.6 & 12.5 \\
\hline 143 & 12.17 & 6.1 & 7.5 & 14.2 & 10.8 & 0.7 & 15.9 & 13.4 & 13.1 \\
\hline 144 & 23.85 & 7.8 & 8.0 & 12.7 & 13.2 & -1.9 & 13.9 & 15.3 & 13.7 \\
\hline 145 & 8.30 & 5.7 & 7.9 & 12.6 & 11.8 & -1.6 & 13.6 & 13.8 & 14.4 \\
\hline 146 & 17.22 & 7.6 & 8.6 & 12.8 & 14.2 & -1.2 & 13.3 & 15.5 & 15.0 \\
\hline 148 & 5.73 & 7.3 & 9. & 12.7 & 15. & -0.6 & 12. & 16.1 & * \\
\hline 150 & 5.62 & 7.3 & * & 13. & * & 0.3 & $12 \cdot 3$ & 16. & * \\
\hline
\end{tabular}

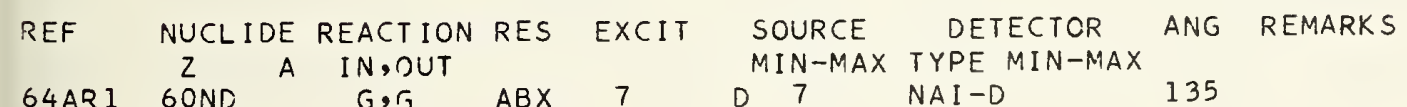

$\begin{array}{lllllllll}64 A R I & 60 N D & G, r_{3} & A B X & 7 & D & 7 & \text { NAI-D } & 135 \\ 59 H A 2 & 60 N D & G, A & & & & & \text { EMU }\end{array}$

59CA3 6OND142 G,N ABX 1032 C $10-32$ ACT-I 4PI

$\begin{array}{llllllll}63 B L 2 & 60 N D 144 & G, G & \text { ABX } & 2 & D & 2 & \\ 63 R I 2 & 60 N D 144 & G, G & \text { LFT } & 2 & D & 2 & \text { NAI-D }\end{array}$

60GE3 6ONDI45 G,N NOX THR CTHR BF3-I

$59 C A 360 N D 150 \mathrm{G}, \mathrm{N} \quad \mathrm{RLX} \quad 8 \quad 32 \quad \mathrm{C} \quad 8-32$ ACT-I
90 G-WDTH, SEP ISOTPS
4PI THRESHOLD

4P I 


\begin{tabular}{|c|c|c|c|c|c|c|c|c|c|}
\hline A & ABUND. & & SE & ATION & ENERGIES & (MEV) & & & \\
\hline & & $G, N$ & $G, P$ & $G, T$ & $G, H E 3$ & $G, A$ & $G, 2 N$ & $G, N P$ & $G, 2 P$ \\
\hline 144 & 3.00 & 10.5 & 6.4 & 16.3 & 12.6 & 0 & * & 16.2 & 10.5 \\
\hline 147 & 14.97 & 6.3 & 7.1 & 12. & $10 \cdot 4$ & $-2 \cdot 3$ & 14.8 & 13.4 & 12.4 \\
\hline 148 & 11.24 & 8.1 & 7.6 & 13.0 & 12.8 & -2.0 & 14.5 & 15.3 & 13.0 \\
\hline 149 & 13.83 & 5.8 & 7.5 & 12.6 & 11.1 & -1.9 & 14.0 & 13.5 & 13.5 \\
\hline 150 & 7.44 & 8.0 & $8 \cdot 3$ & 12.9 & 13.8 & -1.5 & 13.8 & 15.5 & 14.2 \\
\hline 152 & 26.72 & 8.2 & 8.6 & 13.6 & 15.3 & -0.3 & 13.8 & 16.5 & 15.6 \\
\hline 154 & 22.71 & 7.9 & 8.9 & 13.9 & $16 \cdot 3$ & 1.1 & 13.8 & 16 & * \\
\hline
\end{tabular}

\begin{tabular}{|c|c|c|c|c|c|c|c|c|c|c|}
\hline REF & $\begin{array}{r}\text { NUCL IDE } \\
Z \quad A\end{array}$ & $\begin{array}{l}\text { REACT ION } \\
\text { IN,OUT }\end{array}$ & RES & EX & CIT & & $\begin{array}{l}\text { OURCE } \\
\text { IN-MAX }\end{array}$ & $\begin{array}{c}\text { DETECTOR } \\
\text { TYPE MIN-MAX }\end{array}$ & ANG & REMARKS \\
\hline 6ORE I & $62 \mathrm{SM}$ & $G, G$ & $A B X$ & 7 & & D & 7 & NAI -D & 90 & \\
\hline $62 B E 2$ & $625 M$ & $G, G$ & $A B X$ & 5 & 9 & $D$ & $5-9$ & $N A I-D$ & 135 & \\
\hline $64 A R 1$ & $625 \mathrm{MI}$ & $G, G$ & $A B X$ & 9 & & D & 9 & $N A I \rightarrow D$ & 135 & \\
\hline 59011 & $625 M$ & $G, N$ & $A B X$ & & & & & $A C T-I$ & $4 \mathrm{PI}$ & * \\
\hline $58 \mathrm{FUl}$ & $625 M$ & $G, \times N$ & RLX & & -40 & $c$ & $7-40$ & BF 3-I & $4 \mathrm{PI}$ & \\
\hline $59 H A 2$ & $625 M$ & $G, A$ & & & & & & EMU & & $\star$ \\
\hline $55 D E 1$ & $62 S M 144$ & $G, N$ & $A B X$ & 9 & 23 & C & $9-23$ & $A C T-I$ & 4PI & \\
\hline 56 SI 1 & $62 \sin 144$ & $G, N$ & $A B X$ & 9 & 22 & $c$ & 22 & $A C T-I$ & $4 \mathrm{PI}$ & THRESHOLD \\
\hline $59 C A 3$ & $62 \operatorname{SM1} 44$ & $G, N$ & $A B X$ & 10 & 32 & $c$ & $10-32$ & $A C T-I$ & $4 \mathrm{PI}$ & \\
\hline OGE3 & 62 SMI 149 & $G, N$ & NOX & THR & & & $H R$ & $B F 3-I$ & $4 \mathrm{PI}$ & THRESHOLD \\
\hline $9 \subset A 3$ & E2SMI 154 & $G, N$ & $A B X$ & 8 & 32 & $c$ & $8-32$ & $A C T-1$ & $4 \mathrm{PI}$ & \\
\hline $2 \mathrm{KO} 3$ & $62 \sin 154$ & $G, P$ & RLX & THR & 20 & $c$ & 20 & $A C T-I$ & $4 \mathrm{PI}$ & REL TO NE \\
\hline
\end{tabular}

EUROPIUM $Z=63$

\begin{tabular}{|c|c|c|c|c|c|c|c|c|c|}
\hline A & ABUND. & & SEF & ATION & ENERGIES & (MEV) & & & \\
\hline & & $G, N$ & $G, P$ & $G, T$ & $G, H E 3$ & $G, A$ & $\mathrm{G}, 2 \mathrm{~N}$ & $G, N P$ & $G, 2 P$ \\
\hline $\begin{array}{l}151 \\
153\end{array}$ & 47.82 & 7.9 & $4 \cdot 9$ & 10.2 & 12.7 & $\begin{array}{l}-2 \cdot 0 \\
-0.3\end{array}$ & 14.4 & $12 \cdot 9$ & $\begin{array}{l}13.1 \\
14.5\end{array}$ \\
\hline
\end{tabular}

REF NUCLIDE REACTION RES EXCIT SOURCE DETECTOR ANG REMARKS $Z$ A IN,OUT MIN-MAX TYPE MIN-MAX 6OGE3 63EUI5I G,N NOX THR CTHR BF3-I 4PI THRESHOLD 6OGE3 63EUI53 G,N NOX THR CTHR BF3-I 4PI THRESHOLD 


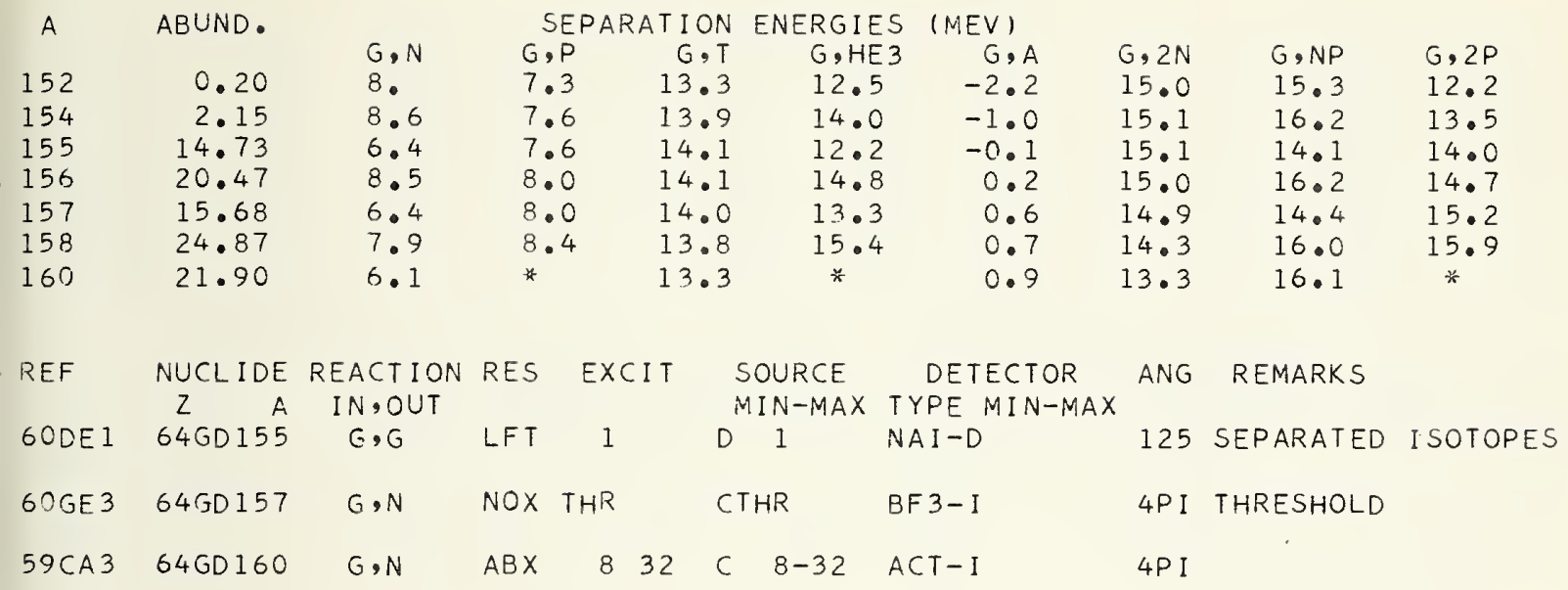

TERBIUM $Z=65$

A ABUND. SEPARATION ENERGIES (MEV)

$\begin{array}{rrrlllrll} & & G, N & G, P & G, T & G, H E 3 & G, A & G, 2 N & G, N P \\ 156 & 5.2(-2) & 10 . & 7 . & 14 . & 12.7 & -1.4 & 16.5 & 16.2 P \\ 159 & 99.95 & 8.2 & 6.2 & 12.0 & 14.4 & 0.2 & 14.9 & 14.2\end{array}$

\begin{tabular}{|c|c|c|c|c|c|c|c|c|}
\hline REF & NUCL IDE & REACT ION & RES & EXCI T & SOURCE & DETECTOR & ANG & REMARKS \\
\hline & Z A & IN,OUT & & & MIN-MAX & TYPE MIN-MAX & & \\
\hline 59ME I & $65 T 3$ & $G, G$ & LFT & 1 & D 1 & NAI -D & DST & MULTIPOLAR I TY \\
\hline 64LA 1 & $65 T B$ & $G, G$ & $A B X$ & 1025 & $c$ & NAI -D & DST & \\
\hline 64BR I & $65 \mathrm{~TB}$ & $G, N$ & $A B X$ & $8-28$ & $8-28$ & $B F 3-I$ & $4 P I$ & \\
\hline $64 B R 1$ & 65 TB & $G, 2 N$ & $A B X$ & $14-28$ & $8-28$ & $B F 3-I$ & 4PI & \\
\hline $58 F \cup 1$ & $65 T B$ & $G, \times N$ & $A B Y$ & $7-40$ & $7-40$ & BF 3-I & 4PI & \\
\hline $58 F \cup 2$ & $65 \mathrm{~TB}$ & $G, X N$ & $A B X$ & $8-23$ & CTHR -25 & BF 3-I & $4 P I$ & \\
\hline $58 \mathrm{CH} 2$ & 65 TB 159 & $G, N$ & RLY & THR & CTHR & $B F 3-I$ & $4 P I$ & THRESHOLD \\
\hline 6OGE 3 & $65 T 3159$ & $G, N$ & NOX & THR & CTHR & BF 3-I & 4PI & THRESHOLD \\
\hline $62 \mathrm{BO} 1$ & 65 TB 159 & $G, N$ & $A B X$ & 821 & CTHR -21 & BF 3-I & $4 P I$ & \\
\hline $58 \mathrm{KAI}$ & 65 TB 159 & $G, \times N$ & $A B X$ & 922 & $9-22$ & BF 3-I & 4PI & \\
\hline $60 T H 1$ & $65 T B 159$ & $G, \times N$ & $A B X$ & 818 & $7-18$ & BF 3-I & 4PI & \\
\hline
\end{tabular}




\begin{tabular}{|c|c|c|c|c|c|c|c|c|c|}
\hline A & ABUND. & & SEF & AT ION & ENERGIES & (MEV) & & & \\
\hline & & $G, N$ & $G, P$ & $G, T$ & G, HE 3 & $G, A$ & $\mathrm{G}, 2 \mathrm{~N}$ & $G, N P$ & $G, 2$ \\
\hline 156 & $5 \cdot 2(-2)$ & 7.1 & * & 12.9 & * & 0.6 & 12.8 & 15.8 & 12 \\
\hline 158 & $9.0(-2)$ & 9. & 6.9 & 14. & 13.3 & -0.8 & 15.7 & 16. & 12 \\
\hline 160 & 2.29 & 8.6 & 7.4 & 13.9 & 13.8 & -0.4 & 15.4 & 15.6 & \\
\hline 161 & 18.88 & 6.4 & 7.5 & 13.6 & 12.3 & -0.3 & 15.0 & 13.9 & 4. \\
\hline 162 & 25.53 & 8.2 & 8.0 & 13.6 & 14.5 & 0.0 & 14.6 & 15.7 & 4 \\
\hline 163 & 24.97 & 6.2 & $8 \cdot 3$ & 13.4 & 13.4 & 0.2 & 14.5 & .3 & \\
\hline 164 & 28.18 & 7 & 8.5 & 13.4 & 15.4 & 0.5 & 13.9 & 16.0 & 5. \\
\hline
\end{tabular}

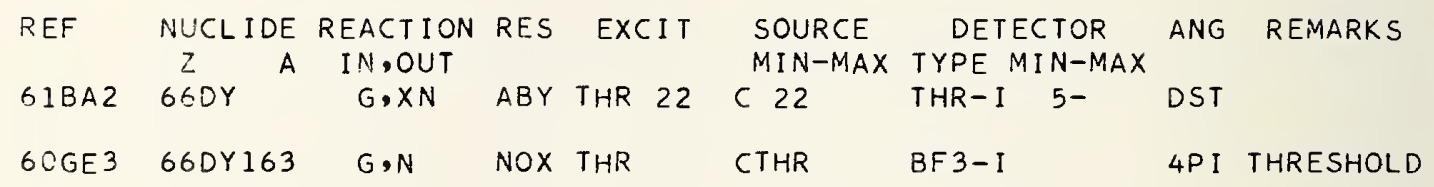

HOLMIUM $Z=67$

\begin{tabular}{|c|c|c|c|c|c|c|c|c|}
\hline A & ABUND. & & SEF & ATION & ENERGIES & (MEV) & & \\
\hline 165 & 100.00 & $\begin{array}{l}G, N \\
8.0\end{array}$ & $\begin{array}{l}G, P \\
6.1\end{array}$ & $\begin{array}{r}G, T \\
11.6\end{array}$ & $\begin{array}{l}\text { G,HE3 } \\
14 .\end{array}$ & $\begin{array}{r}G, A \\
-0.2\end{array}$ & $\begin{array}{l}G, 2 N \\
14.6\end{array}$ & $\begin{array}{l}G, N P \\
13.8\end{array}$ \\
\hline
\end{tabular}

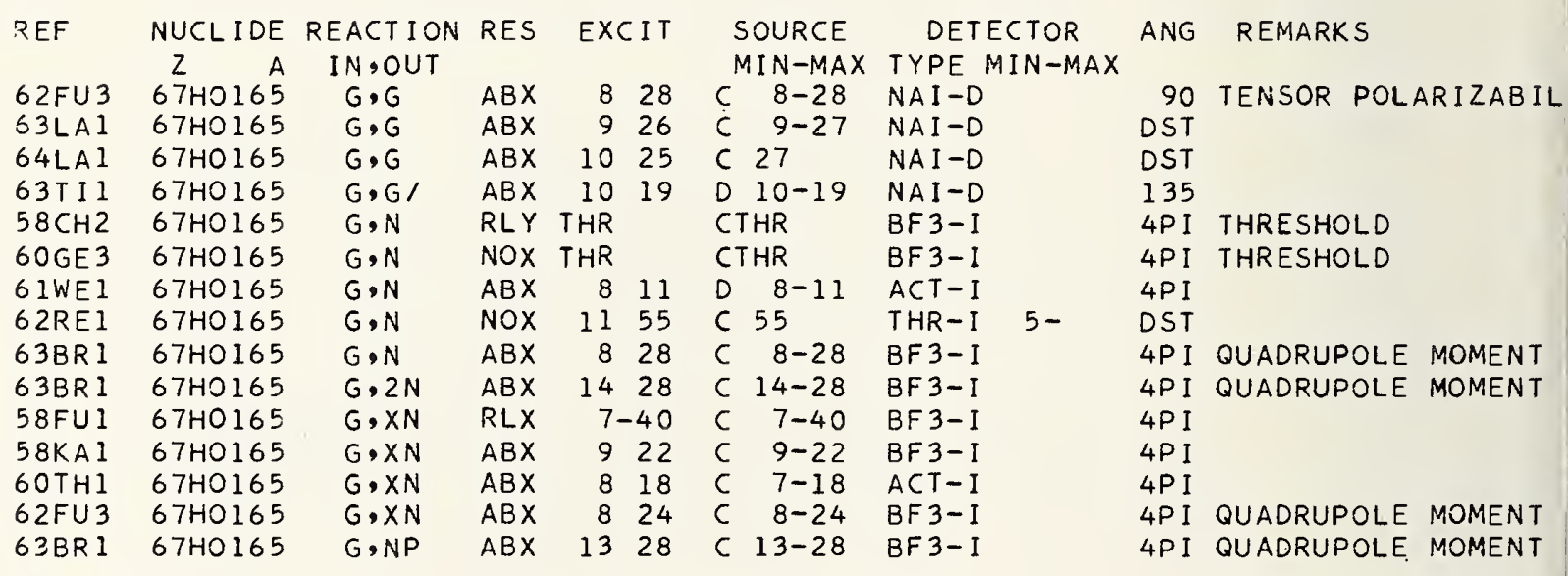




\begin{tabular}{|c|c|c|c|c|c|c|c|c|c|}
\hline A & ABUND. & & $S E$ & TION & ENERGIES & (MEV) & & & \\
\hline & & $G, N$ & $G, P$ & $G, T$ & $G, H E 3$ & $G, A$ & $\mathrm{G}, 2 \mathrm{~N}$ & $G, N P$ & $G$, \\
\hline 62 & 0.14 & 9. & 6. & 14. & $12 \cdot 2$ & -1.5 & * & 15.4 & $\begin{array}{l}11 \\
12\end{array}$ \\
\hline 164 & 1.56 & 8.8 & 6.8 & 14. & 12.8 & $-1 \cdot 3$ & 15.6 & 15.2 & 12. \\
\hline 10 & 33.41 & 8.5 & 7.4 & 13.5 & 13.5 & -0.8 & 15.2 & 15.5 & 3. \\
\hline 167 & 22.94 & 5.4 & 7.5 & 13.4 & $12 \cdot 3$ & -0.6 & 15.0 & 13.9 & \\
\hline 16 & 27.07 & 7.8 & 8.0 & 13.1 & 14.4 & -0.5 & 14.2 & 15.3 & 15. \\
\hline .7 & 14.88 & $7 \cdot 2$ & 8.5 & $12 \cdot 7$ & * & -0.1 & 13.2 & 15.7 & \\
\hline
\end{tabular}

\begin{tabular}{|c|c|c|c|c|c|c|c|c|c|}
\hline REF & $\underset{Z}{N U C L I D E}$ & $\begin{array}{l}\text { REACT ION } \\
\text { IN, OUT }\end{array}$ & RES & EXCIT & \multicolumn{2}{|c|}{$\begin{array}{l}\text { SOURCE } \\
\text { MIN-MAX }\end{array}$} & $\begin{array}{c}\text { DETECTOR } \\
\text { TYPE MIN-MAX }\end{array}$ & ANG & REMARKS \\
\hline $62 F \cup 3$ & 68ER & $G, G$ & $A B X$ & $8 \quad 28$ & $c$ & $8-28$ & NAI -D & 90 & TENSOR POLARIZABIL \\
\hline $63 \mathrm{LAl}$ & 68ER & $G, G$ & $A B X$ & 926 & $C$ & $9-27$ & $N A I-D$ & DST & \\
\hline $64 \mathrm{LA} 1$ & $68 \mathrm{ER}$ & $G, G$ & $A B X$ & $10 \quad 25$ & $c$ & & $N A I-D$ & DST & \\
\hline $63 \mathrm{KA} 2$ & 68ER & $G, G /$ & RLY & 1 & $c$ & 4 & $A C T-I$ & 4PI & \\
\hline $58 \mathrm{FU1}$ & 68ER & $G, \times N$ & $R L X$ & $7-40$ & $c$ & $7-40$ & $B F 3-I$ & 4PI & \\
\hline $62 \mathrm{FU} 3$ & 68ER & $G, \times N$ & $A B X$ & 824 & $c$ & $8-24$ & $B F 3-I$ & $4 \mathrm{PI}$ & QUADRUPOLE MOMENT \\
\hline 60GE3 & 68ER 167 & $G, N$ & NOX & THR & $C T$ & & $B F 3-I$ & 4PI & THRESHOLD \\
\hline $3 M I 3$ & $68 E R 170$ & $G, P$ & NOX & THR 21 & $c$ & 21 & $A C T-I$ & 4PI & \\
\hline
\end{tabular}

THULIUM $Z=69$

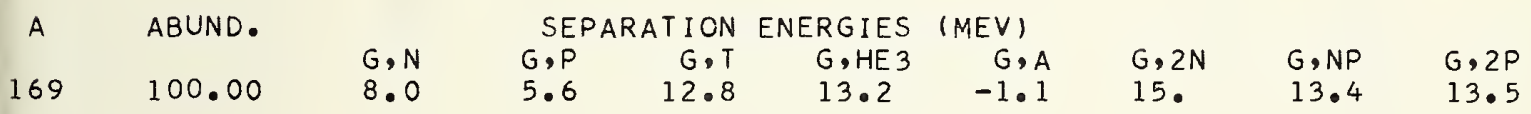

REF

$58 \mathrm{CH} 2$ $60 \mathrm{GE} 3$

$58 \mathrm{KAl}$
NUCLIDE REACTION RES EXCIT $Z$ A IN,OUT $69 T M 169$ 69 TM169 69TM169

$G, N$
$G, N$
$G, X N$

RLY THR
NOX $G, \times N$

\begin{tabular}{ll} 
SOURCE & \multicolumn{1}{c}{ DETECTOR } \\
MIN-MAX & TYPE MIN-MAX \\
CTHR & BF3-I \\
CTHR & BF3-I \\
C $8-22$ & BF $3-I$
\end{tabular}

ANG REMARKS

4PI THRESHOLD 4PI THRESHOLD 4PI

YTTERBIUM $Z=70$

A

\section{8}

170

171

172

173

174

176
ABUND.

0.14
3.03
14.31
21.82
16.13
31.84
12.73

$6, N$
9.
$8 \cdot$
$6 \cdot 8$
$8 \cdot 1$
6.5
7.4
6.6

SEPARATION ENERGIES (MEV)

$\begin{array}{llcrlll}\text { GEP } & G, T & \text { G, HE3 } & \text { G,A } & \text { G,2N } & \text { G,NP } & \text { G.2P } \\ 6 . P & 13 . & 11.8 & -2.1 & 15.9 & 14.8 & 11.0 \\ 6.6 & 13.0 & 12.2 & -2.0 & 15.3 & 14.7 & 12.1 \\ 6.9 & 12.9 & 11.2 & -1.6 & 15.9 & 13.4 & 12.9 \\ 7.4 & 13.0 & 13.3 & -1.3 & 14.9 & 15.1 & 13.8 \\ 7.6 & 11.4 & 12.4 & -0.8 & 14.6 & 14.0 & 14.6 \\ 8.0 & 12.9 & 14.4 & -0.5 & 13.9 & 15.1 & 15.1 \\ 8 . & 12.0 & * & -0.7 & 12.5 & 12.7 & *\end{array}$

REF NUCLIDE REACTION RES EXCIT $Z$ A IN,OUT

$58 F U 1$ 7OYB

6OGE3 7OYB 173
$G, \times N$

$G, N \quad$ NOX THR

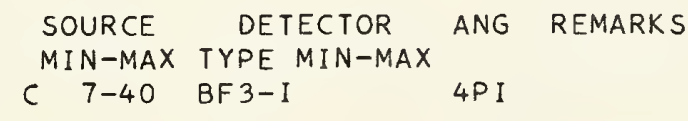

SOURCE DETECTOR ANG REMARKS MIN-MAX TYPE MIN-MAX

C 7-40 BF3-I 4PI

CTHR BF3-I

4PI THRESHOLD 
A ABUND.

175

176
SEPARATION ENERGIES (MEV)

$\begin{array}{rrrr}G, P & G, T & G, H E 3 & G, A \\ 5.5 & 11.0 & 12.8 & -1.4 \\ 5.9 & 10.7 & 12.0 & -1.6\end{array}$

G, $2 \mathrm{~N}$

14.4

14.0
G, NP 13.0

11.7
G, 2P

13.5

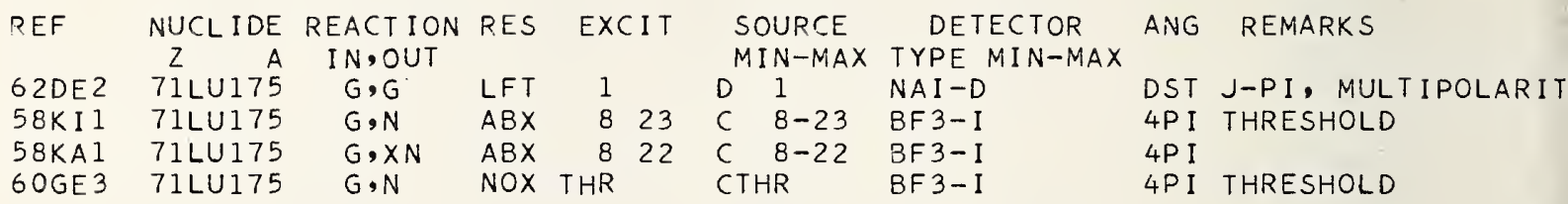

HAFNIUM $Z=72$

\begin{tabular}{|c|c|c|c|c|c|c|c|c|c|}
\hline A & ABUND. & & SE & ATION & ENERGIES & (MEV) & & & \\
\hline & & $G, N$ & G, P & $G, T$ & G, HE 3 & $\begin{array}{r}G, A \\
-2, G\end{array}$ & $\mathrm{G}, 2 \mathrm{~N}$ & $G, N P$ & $G, 2 P$ \\
\hline $\begin{array}{l}174 \\
176\end{array}$ & 0.18 & & $3 \cdot 8$ & & & & & $14 \cdot 4$ & 10.9 \\
\hline 176 & 5.20 & 8. & 6.4 & 12.4 & 11.7 & $-2 \cdot 4$ & 15.0 & 14.3 & 11.9 \\
\hline 177 & 18.50 & 6.4 & 6.6 & $12 \cdot 1$ & 10.6 & $-2 \cdot 5$ & 14. & $12 \cdot 8$ & $12 \cdot 5$ \\
\hline 178 & 27.14 & 7.6 & $7 \cdot 3$ & 11.9 & 12.4 & $-2 \cdot 4$ & 14.0 & $14 \cdot 2$ & 13.5 \\
\hline 179 & 13.75 & 6.1 & 7.5 & 11.8 & 11.8 & $-2 \cdot 1$ & 13.7 & 13.4 & 14.0 \\
\hline 80 & 35.24 & 7.3 & 7.9 & 12.2 & 13.6 & -1.4 & 13.4 & 14.9 & 15 . \\
\hline
\end{tabular}

\begin{tabular}{|c|c|c|c|c|c|c|c|c|c|}
\hline REF & NUCL IDE & REACTION & RES & EXC & IT & SOURCE & DETECTOR & ANG & REMARKS \\
\hline & $Z A$ & IN,OUT & & & & MIN-MAX & TYPE MIN-MAX & & \\
\hline $\begin{array}{l}61 \mathrm{HAl} \\
63 \mathrm{KA} 2\end{array}$ & $72 \mathrm{HF}$ & $G, G$ & LFT & 1 & & $\begin{array}{ll}D & 1 \\
C & 5\end{array}$ & & & * 2 LEVELS \\
\hline $\begin{array}{l}63 K A 2 \\
63 V E 2\end{array}$ & $\begin{array}{l}72 \mathrm{HF} \\
72 \mathrm{HF}\end{array}$ & $\begin{array}{l}G, G / \\
G, G /\end{array}$ & $\begin{array}{l}R L Y \\
A B X\end{array}$ & $\begin{array}{l}1 \\
0\end{array}$ & 1 & $\begin{array}{ll}C & 5 \\
D & 0-1\end{array}$ & $\begin{array}{l}A C T-I \\
N A I-D\end{array}$ & $4 \mathrm{PI}$ & I SOMERS \\
\hline $8 \mathrm{~T}$ & $72 \mathrm{HF} 177$ & $G, N$ & RLY & 6 & 7 & $6-7$ & $8 F 3-I$ & 4PI & THRESHOLD \\
\hline C & $72 \mathrm{HF} 177$ & $G, N$ & NOX & THR & & CTHR & $B F 3-I$ & $4 \mathrm{PI}$ & THRESHOLD \\
\hline $8 \mathrm{~T}$ & $72 \mathrm{HF} 179$ & $G, N$ & RLY & 6 & 7 & $6-7$ & $B F 3-I$ & $4 \mathrm{PI}$ & THRESHOLD \\
\hline$O G$ & $72 \mathrm{HF} 179$ & $G, N$ & NOX & THR & & CTHR & BF 3-I & 4P I & THRESHOLD \\
\hline OGE3 & $72 \mathrm{HF} 180$ & $G, N$ & NOX & THR & & CTHR & $B F 3-I$ & 4PI & THRESHOLD \\
\hline
\end{tabular}




\begin{tabular}{|c|c|c|c|c|c|c|c|c|}
\hline ABUND. & & SEF & ATION & ENERGIES & (MEV) & & & \\
\hline & $G, N$ & $G, P$ & $G, T$ & G,HE3 & $G, A$ & $G, 2 N$ & $G, N P$ & \\
\hline $1 \cdot 2(-2)$ & 6.8 & 5.9 & 11.1 & 11.6 & $-2 \cdot 1$ & 14.6 & 12.0 & $3 \cdot 4$ \\
\hline 99.99 & 7.6 & 6.2 & 11.1 & 13.3 & -1.4 & 14.4 & 13.5 & 14. \\
\hline
\end{tabular}

REF NUCLIDE REACTION RES EXCIT SOURCE DETECTOR ANG REMARKS

\begin{tabular}{|c|c|c|c|c|c|c|c|c|c|c|}
\hline & $Z \quad A$ & IN, OUT & & & & MIN-MAX & TYPE I & IN-MAX & & \\
\hline $58 F \cup 3$ & 73TA & $G, G$ & $A B X$ & & -27 & C $\quad 5-27$ & $N A I-D$ & & 120 & \\
\hline 64LAI & 73Т A & $G, G$ & $A B X$ & 10 & 25 & C 32 & NAI I D & & DST & \\
\hline $55 \mathrm{JOl}$ & 73TA & $G, N$ & RLY & THR & 65 & C 65 & $S C I-D$ & $5-$ & DST & \\
\hline 5001 & 73Т A & $G, N$ & RLY & THR & 65 & C 65 & $S C I-D$ & $10-$ & DST & \\
\hline $4 G R 2$ & 73TA & $G, N$ & $A B X$ & THR & 11 & DTHR-11 & $B F 3-I$ & & $4 \mathrm{PI}$ & \\
\hline $8 \mathrm{FUI}$ & 73TA & $G, X N$ & $A B Y$ & & -40 & C $7-40$ & $B F 3-I$ & & 4PI & \\
\hline $58 F U 2$ & 73TA & $G, \times N$ & $A B X$ & & -23 & CTHR -25 & $B F 3-I$ & & 4PI & \\
\hline 58BA4 & $73 \mathrm{TA}$ & $G, X P$ & RLX & THR & 40 & -40 & $M A G-D$ & & DST & * SPECTRUM \\
\hline $56 G A 1$ & 73TA 181 & $G, M U-T$ & $A B I$ & 8 & 27 & $8-27$ & BF 3-I & & & \\
\hline $631 \mathrm{Al}$ & 73 TA 131 & $G, G$ & NOX & 11 & 22 & C 27 & NAI I D & & DST & \\
\hline $63 Y 01$ & 73TA181 & $G, G$ & $A B X$ & 6 & 8 & D $\quad 6-8$ & NAI I D & & 120 & \\
\hline $57 C A 1$ & 73ТA 181 & $G, N$ & RLX & 8 & 31 & $8-31$ & $A C T-I$ & & 4PI & \\
\hline $57 F E 2$ & 73TA 181 & $G, N$ & RLY & 14 & 30 & C $14-30$ & THR-I & & 90 & \\
\hline $58 \mathrm{BE} 2$ & 73 TA 181 & $G, N$ & $S P C$ & 8 & 16 & C 14,16 & $T O F-D$ & $1-7$ & 120 & \\
\hline $58 C A 1$ & 73ТА.181 & $G, N$ & $A B X$ & 7 & 32 & C $7-32$ & $A C T-I$ & & $4 \mathrm{PI}$ & THRESHOLD \\
\hline $58 \mathrm{CH} 2$ & 73 TA 181 & $G, N$ & RLY & THR & & CTHR & BF 3-I & & $4 \mathrm{PI}$ & THRESHOLD \\
\hline $58 \mathrm{COl}$ & 73ТA 181 & $G, N$ & $S P C$ & 9 & 30 & C 20,30 & EMU-D & $2-18$ & 90 & THRESHOLD \\
\hline $58 S P 1$ & 73TA18! & $G, N$ & $A B X$ & 8 & 18 & C $8-18$ & $B F 3-I$ & & $4 P I$ & \\
\hline $59 A \cup 1$ & 73TA181 & $G, N$ & $A B X$ & 18 & 65 & C $18-65$ & THR-I & $5-$ & 4P I & \\
\hline $59 C A 3$ & 73TA 181 & $G, N$ & $A B X$ & 8 & 33 & $8-33$ & $A C T-I$ & & DST & \\
\hline 59PAI & 73TA181 & $G, N$ & $A B X$ & 8 & 22 & $8-22$ & BF 3-I & & 4PI & THRESHOLD \\
\hline 60BA2 & $73 \mathrm{TAl} 81$ & $G, N$ & $R L X$ & 8 & 32 & $8-32$ & $A C T-I$ & & 4PI & \\
\hline $60 G E 3$ & 73TA181 & $G, N$ & NOX & THR & & CTHR & $A C T-I$ & & 4PI & THRESHOLD \\
\hline OIWEI & 73ТA181 & $G, N$ & $A B X$ & 7 & 11 & $7-11$ & $A C T-I$ & & $4 \mathrm{PI}$ & THRESHOLD \\
\hline 62801 & 73 TA181 & $G, N$ & $A B X$ & 9 & 23 & $9-23$ & BF 3-I & & 4P I & \\
\hline $62 M I 3$ & 73 TA181 & $G, N$ & $A B X$ & 7 & 22 & $7-22$ & BF 3-I & & 4PI & \\
\hline $63 B R 1$ & 73TA181 & $G, N$ & $A B X$ & 8 & 28 & $8-28$ & BF 3-I & & 4PI & QUADRUPOLE MOMENT \\
\hline $632 \mathrm{Al}$ & 73 TA 181 & $G, N$ & SPC & 9 & 19 & C 14,19 & $E M U-D$ & $2-12$ & DST & \\
\hline $60 \mathrm{BA} 2$ & 73TA181 & $E, N$ & RLY & 8 & 42 & $8-42$ & $A C T-I$ & & 4PI & REL TO $\mathrm{G}, \mathrm{N}$ \\
\hline $57 \mathrm{CAI}$ & 73 TA 181 & $G, 2 N$ & RLX & 14 & 31 & $8-31$ & MOD-I & & 4P I & MIXED WITH G, $3 N$ \\
\hline $58 C A 1$ & 73TA181 & $G, 2 \mathrm{~N}$ & $A B X$ & 14 & 32 & $7-32$ & $A C T-I$ & & 4PI & THRESHOLD \\
\hline $638 \mathrm{R} 1$ & 73TA 181 & $G, 2 N$ & $A B X$ & 13 & 28 & $8-28$ & $B F 3-I$ & & 4PI & QUDRPL MOM, THRESH \\
\hline 57CA 1 & 73TA 181 & $\mathrm{G}, 3 \mathrm{~N}$ & RLX & 14 & 31 & $8-31$ & MOD-I & & $4 \mathrm{PI}$ & MIXED WITH $G, 2 \mathrm{~N}$ \\
\hline $58 C A 1$ & 73 TA181 & $G, 3 N$ & $A B X$ & 22 & 32 & $7-32$ & $A C T-I$ & & 4P I & THRESHOLD \\
\hline $6 C B A 2$ & 73 TA 181 & $G, 3 N$ & $R L X$ & 22 & 32 & c $22-32$ & $A C T-I$ & & 4P I & \\
\hline 60BA2 & 73 TA181 & $E, 3 N$ & RLY & 22 & 42 & D $22-42$ & $A C T-I$ & & 4PI & REL TO $G, 3 N$ \\
\hline $56 G A 1$ & 73TA 181 & $G, X N$ & $A B X$ & 8 & 27 & $8-27$ & $B F 3-I$ & & 4P I & \\
\hline $56 \mathrm{HAl}$ & 73TA181 & $G, \times N$ & $A B X$ & 15 , & 18 & D 15,18 & BF 3-I & & 4PI & \\
\hline 57 CA 1 & 73 TA 181 & $G, \times N$ & RLX & 8 & 31 & $8-31$ & MOD-I & & $4 \mathrm{PI}$ & \\
\hline $58 \mathrm{KAl}$ & 73TA 181 & $G, \times N$ & $A B X$ & 9 & 22 & $9-22$ & $B F 3-I$ & & 4PI & \\
\hline $61 \mathrm{BA2}$ & 73TA181 & $G, \times N$ & ABY & THR & 22 & C 22 & THR-I & $3-$ & DST & \\
\hline $61 B A 2$ & 73TA181 & $G, \times N$ & $A B Y$ & THR & 22 & C 22 & THR-I & $5-$ & DST & \\
\hline $61 M I 1$ & 73TA181 & $G, \times N$ & $A B X$ & 6 & 22 & D $6-22$ & $B F 3-I$ & & 4P I & \\
\hline $63 B R I$ & 73TA 181 & $G, N P$ & $A B X$ & 14 & 28 & $8-28$ & BF 3-I & & $4 \mathrm{PI}$ & \\
\hline 59SE2 & 73TA 181 & $G, P$ & $A B X$ & 15 , & 18 & D 15,18 & $E M U-D$ & & & * \\
\hline $60 B A 5$ & 73TA181 & $G, P$ & $A B X$ & 17 & 39 & C 17-30 & $M A G-D$ & $6-22$ & DST & SPECTRUM \\
\hline $60 C A 2$ & 73 TA 181 & $G, P$ & $A B X$ & 15 & 32 & C $15-32$ & $A C T-I$ & & 4PI & THRESHOLD \\
\hline $55 \mathrm{TOI}$ & 73TA181 & $G, X P$ & SPC & 11 & 23 & C 23 & $E M U-D$ & $5-18$ & DST & ABSOLUTE YIELD \\
\hline $60 \mathrm{CHI}$ & 73TA 181 & $G, X P$ & RLX & 22 & 90 & C 90 & TEL-I & $15-30$ & 90 & REL TO DEUTERONS \\
\hline $60 \mathrm{CHI}$ & 73 TA 181 & $G, \times D$ & $R L X$ & 27 & 90 & C 90 & TEL-I & $15-30$ & 90 & REL TO PROTONS \\
\hline $60 \mathrm{CHI}$ & 73TA181 & $G, \times T$ & RLY & 27 & 90 & C 90 & TEL-I & $15-30$ & 90 & REL TO DEUTERONS \\
\hline $57 E R 1$ & 73TA181 & $G, A$ & $A B I$ & 1 & 32 & C 32 & $A C T-I$ & & 4P I & \\
\hline
\end{tabular}




\begin{tabular}{|c|c|c|c|c|c|c|c|c|c|}
\hline 180 & $\begin{array}{r}\text { ABUND. } \\
0.14\end{array}$ & $\underset{*}{G, N}$ & $\begin{array}{l}\text { SEF } \\
G, P \\
6.5\end{array}$ & $\begin{array}{r}\text { TION } \\
\text { G, T } \\
12.8\end{array}$ & $\begin{array}{c}\text { ENERG IES } \\
\text { G, HE } 3 \\
11.6\end{array}$ & $\begin{array}{l}(\text { MEV) } \\
\text { G, A } \\
-2.6\end{array}$ & $\begin{array}{c}G, 2 \mathrm{~N} \\
*\end{array}$ & $\begin{array}{l}\text { G,NP } \\
14.4\end{array}$ & $\begin{array}{l}G, 2 P \\
11.7\end{array}$ \\
\hline $\begin{array}{l}182 \\
183 \\
184\end{array}$ & $\begin{array}{l}26.41 \\
14.40 \\
30.64\end{array}$ & $\begin{array}{l}8.0 \\
6.2 \\
7.4\end{array}$ & $\begin{array}{l}7.0 \\
7.1 \\
7.7\end{array}$ & $\begin{array}{l}13.0 \\
12.4 \\
12.1\end{array}$ & $\begin{array}{l}12.8 \\
11.7 \\
13.1\end{array}$ & $\begin{array}{l}-1.7 \\
-1.6 \\
-1.5\end{array}$ & $\begin{array}{l}14.9 \\
14.2 \\
13.6\end{array}$ & $\begin{array}{l}14.7 \\
13.2 \\
14.6\end{array}$ & $\begin{array}{l}13.2 \\
13.4 \\
14.3\end{array}$ \\
\hline 186 & 28.41 & 7.2 & 8.3 & $12 \cdot 2$ & 14.4 & $-1 \cdot 1$ & 13.0 & 15.0 & * \\
\hline
\end{tabular}

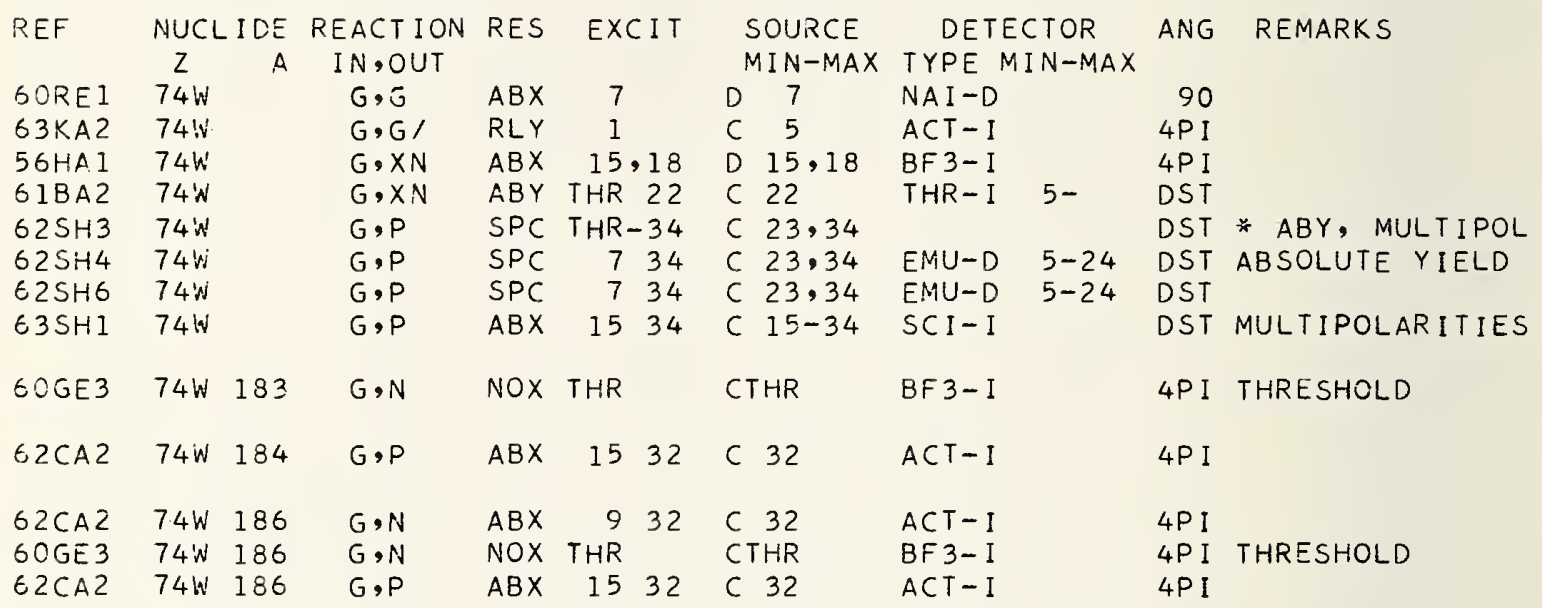

RHEN IUM $Z=75$

\begin{tabular}{|c|c|c|c|c|c|c|c|c|c|}
\hline A & ABUND. & & SE & AT ION & ENERGIES & (MEV) & & & \\
\hline & & $G, N$ & $G, P$ & $G, T$ & $\mathrm{G}, \mathrm{HE} 3$ & $G, A$ & $G, 2 N$ & $G, N P$ & $G, 2 P$ \\
\hline 85 & 37.07 & 8 & $5 \cdot 4$ & 10.5 & 12.2 & $-2 \cdot 3$ & 14. & 12.9 & 13.1 \\
\hline 87 & 52.93 & 7.3 & 6.0 & 10.5 & $13 \cdot 2$ & -1.6 & $13 \cdot 5$ & $13 \cdot 2$ & 14.3 \\
\hline
\end{tabular}

REF NUCLIDE REACTION RES EXCIT SOURCE DETECTOR ANG REMARKS 2 A IN,OUT MIN-MAX TYPE MIN-MAX 64LA3 75REI87 G,G LFT O 1 D $0-1$ NAI-D 60GE3 75RE187 G,N NOX THR CTHR BF3-I THRESHOLD 


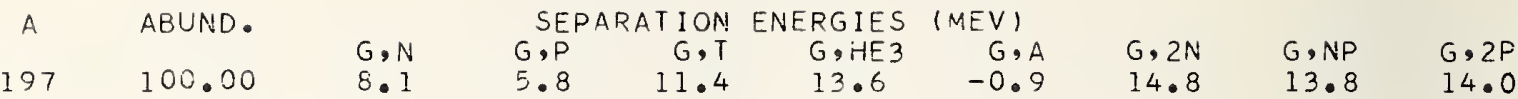

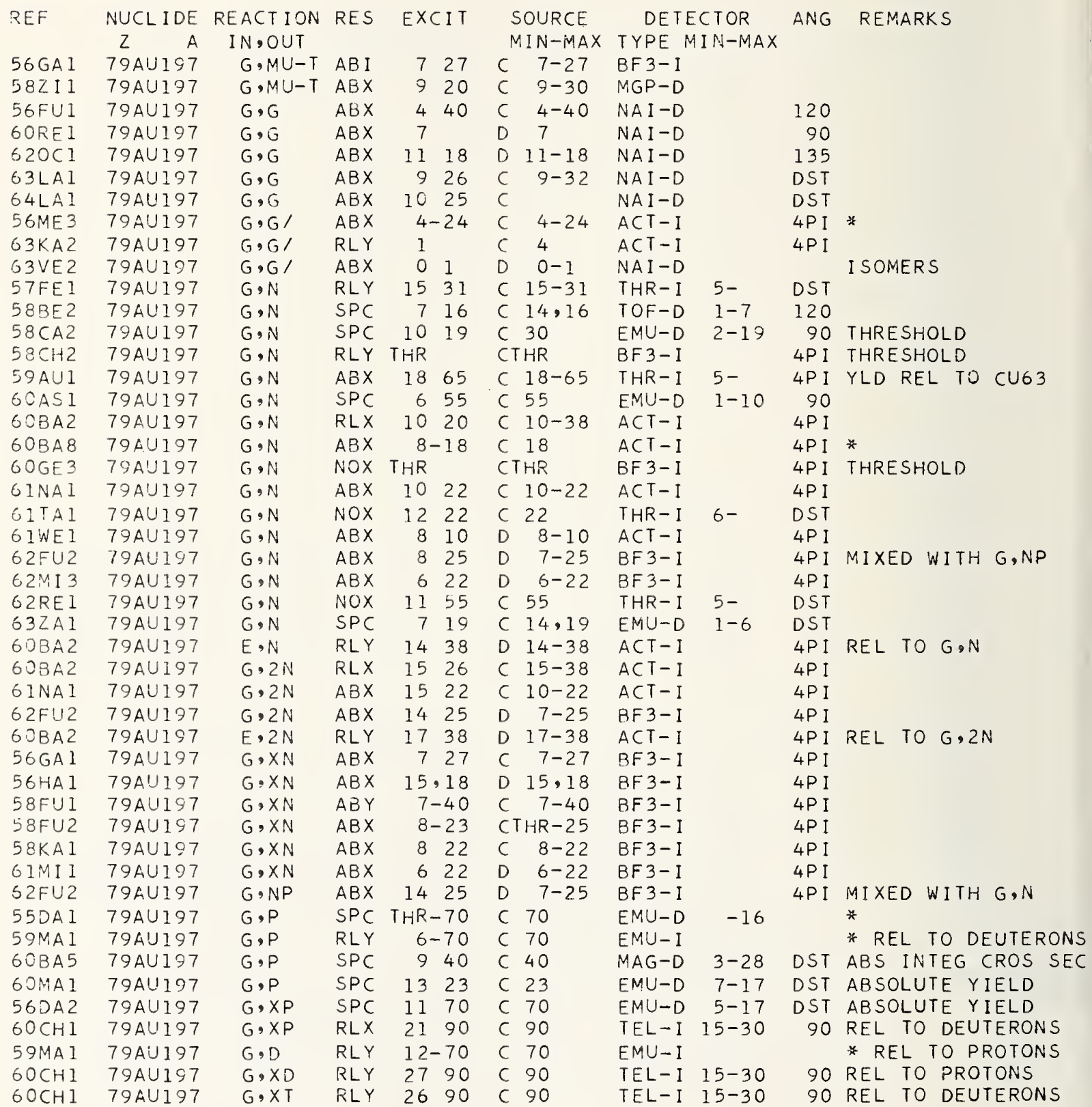


61DE3 81TL203

5TERI 81TL203

5TER $1 \quad 81$ TL203

60GE3 81TL205

57ER1 81TL205

5TER1 81TL205
$G, G$

$G, N A \quad A B I$

$G, A \quad A B I$

$G, N$ NOX THR

GNNA ABX $29 \quad 32$

$G, A \quad A B X \quad 18 \quad 32$

\section{MIN-MAX TYPE MIN-MAX}

D 1 NAI -D

C $32 \quad A C T-I$

C 32 ACT $-I$

CTHR BF3-I

C $18-32$ ACT-I

C $18-32$ ACT-I
DST REL CROSS SECTION

4PI MIXED WITH G, A

4PI MIXED WITH G,NA

4PI THRESHOLD

4P I

4P I

LEAD $Z=82$

\begin{tabular}{|c|c|c|c|c|c|c|c|c|c|}
\hline \multirow[t]{2}{*}{ A } & \multicolumn{2}{|c|}{ ABUND. (1) } & \multicolumn{2}{|c|}{ SEPARATION } & \multicolumn{2}{|c|}{ ENERGIES (MEV) } & \multirow[b]{2}{*}{$\mathrm{G}, 2 \mathrm{~N}$} & \multirow[b]{2}{*}{$G, N P$} & \multirow{2}{*}{$G, 2 P$} \\
\hline & & $G, N$ & $G, P$ & $G, T$ & G, HE3 & $G, A$ & & & \\
\hline 204 & 1.48 & $8 \cdot 2$ & 6.6 & 12.8 & 12.4 & $-2 \cdot 0$ & 15.2 & 14.4 & 12. \\
\hline 06 & 23.6 & 8.1 & 7.3 & 13.0 & 13.4 & -1.1 & 14.8 & 14.8 & 3 . \\
\hline 07 & 22.6 & 6.7 & 7.5 & 13.0 & 12.7 & -0.4 & 14.8 & 14.0 & 14. \\
\hline 08 & 52.3 & 7.4 & 8.0 & 12.9 & 14.5 & -0.5 & 14.1 & 14.9 & 5. \\
\hline
\end{tabular}

(1) ABUNDANCE DEPENDS ON SOURCE

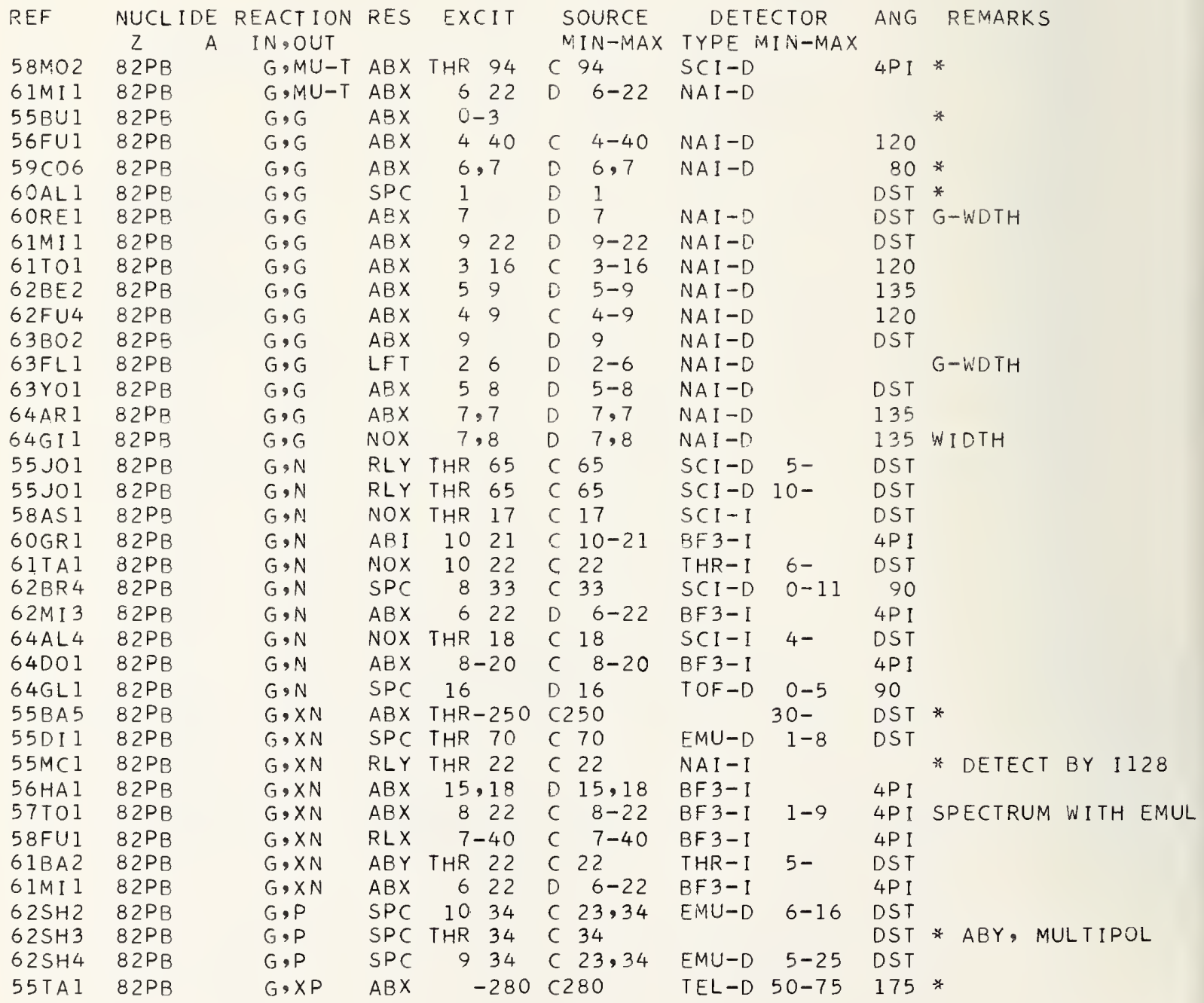


REF

6ORE 1

62 FU 4

$63 \mathrm{~A} \times 1$

60 GE 3

$64 \mathrm{HA} 2$

$64 \mathrm{HA} 2$

62 FU 4

6OGE3

$63 B E 4$

$64 \mathrm{HA} 2$

$64 \mathrm{HA} 2$

$62 \mathrm{FU} 4$

$605 T 1$

55DE 1

62501

$63 \mathrm{~A} \times 1$

63 FL2

64 AR 1

6OBA 4

$61 C R I$

56PR 1

$58 F A 1$

6OGE 3

63 EE 4

$64 \mathrm{HA} 2$

$64 \mathrm{HA} 2$

$62 \mathrm{FU} 4$

62501

$550 E 1$

62501

$55 \mathrm{TOI}$

62501
NUCL IDE
$Z$

82PB206

82 PB 206

82 PB 206

82 PB 206

$82 P B 206$

82 PB206

$82 \mathrm{~PB} 206$

82 PE 207

82 PB 207

82 PB 207

$82 \mathrm{~PB} 207$

82PB207

82 PB 207

82 PB 207

82 PB 207

82 PB 208

82 PB208

$82 \mathrm{~PB} 208$

82 PB 208

$82 P B 208$

$82 \mathrm{~PB} 208$

82 PB 208

82 PB209

$82 P B 208$

82 PB 208

82 PB 208

82 PB 208

82 PB 208

$82 \mathrm{~PB} 208$

82 PB 208

82 PB 208

82 PB 208

\section{IN, OUT}

$G, G$

$G, G$

$G, G$

$G, N$

$G, N$

$G, 2 N$

$G, X N$

$G, N$

$G, N$

$G, N$

$G, 2 N$

$G, X N$

$G, N P$

$G, P$

$G, P$

$G, G$

$G, G$

$G, G$

$E, E /$

$E, E /$

$G, N$

$G, N$

$G, N$

$G, N$

$G, N$

$G, 2 N$

$G, X N$

$G, N P$

$G, P$

$G, P$

$G, X P$

$G, D$
EXCI T

$A B X$

$A B X$

$A B X$

NOX THR

$A B X \quad 627$

$A B X \quad 1227$

$A B X$

819

NOX THR

SPC 7

$A B X \quad 6 \quad 27$

$A B X \quad 1227$

$A B X \quad 619$

RLX 144320

$A B X$

113

$A B X$

1533

$A B X$

LFT

$A B X$

$S P C$

NOX

$$
68
$$

38

$7-8$

- 23

08

722

NOX THR

SPC 710

$A B X \quad 627$

$A B X \quad 12 \quad 27$

$A B X \quad 719$

$A B X \quad 15 \quad 33$

$\begin{array}{ll}15 & 33 \\ 11 & 21\end{array}$

$\begin{array}{ll}11 & 21 \\ 15 & 33\end{array}$

1323

1533
SOURCE

MIN-MAX

$\begin{array}{ll}\text { D } & 7 \\ \text { C } & 4-9 \\ \text { D } & 6-8 \\ \text { CTHR }\end{array}$

D) 6-26

D 12-26

C $8-19$

CTHR

C 9

D $6-26$

D $12-26$

c $6-19$

C 320

c 11-21

c 15-33

D $6-8$

D 7

D $7-8$

D 43

D 183

C 22

C $10-20$

CTHR

C 9,10

D $6-26$

D 12-26

c 7-19

c 15-33

C 11-21

c 15-33

C 23

C 15-33
DETECTOR TYPE MIN-NAX

\section{NAI -D}

NAI -D

NAI $-D$

BF 3-I

BF $3-I$

$B F 3-I$

BF 3-I

$B F 3-I$

TOF $-D \quad 0-2$

BF 3- I

$B F 3-I$

$B F 3-I$

TEL-D 130

$A C T$ - I

$A C T-I$

NA I - D

$S C I-D$

NAI -D

$M A G-D \quad 20-43$

$M A G-D$

NAI - I

ACT - I

$B F 3-I$

TOF $-D$

$B F 3-I$

BF 3-I

BF 3-I

$A C T-I$

ACT - I

$A C T-I$

EMU-D

ACT - I

$0-3$
ANG REMARKS

DST G-WDTH, RADIOLEAD

120 RADIOLEAD

135

4 PI THRESHOLD

4PI

$4 P$ I

4PI

4PI THRESHOLD

4P I

$4 \mathrm{PI}$

$4 \mathrm{PI}$

76 REL TO H2 CROS SEC 4PI MIXED WITH PB208

4PI MIXED WITH PB208

135

4PI G-WDTH

135 WIDTH

160 ABS INTEGR CROSSEC

DST J-PI, TRANS RATE

90 SEPARATED I SOTOPES

4PI I SOMERIC PRODUCT

4PI THPESHOLD

4P I

4P I

$4 P I$

4PI ALSO(G,D),PB207G,P

4PI MIXED WITH PB207

4PI MIXED WITH PB207

5-17 DST SEPARATED ISOTOPES

4PI ALSO G,NP,PB207G,P 


\begin{tabular}{|c|c|c|c|c|c|c|c|c|}
\hline & & & SE & TION & ENERG IES & (MEV) & $6,2 \mathrm{~N}$ & NP \\
\hline 09 & 100.00 & 7.4 & 3.8 & 9.4 & 10.9 & $-3 \cdot 1$ & $14 \cdot 3$ & $11 \cdot 2$ \\
\hline
\end{tabular}

\begin{tabular}{|c|c|c|c|c|c|c|c|c|c|c|c|}
\hline REF & $\begin{array}{r}\text { NUCL IDE } \\
Z\end{array}$ & $\begin{array}{l}\text { REACT ION } \\
\text { IN, OUT }\end{array}$ & RES & EXC & I T & & $\begin{array}{l}\text { OURCE } \\
\text { IN-MAX }\end{array}$ & $\begin{array}{l}\text { DETE } \\
\text { TYPE }\end{array}$ & $\begin{array}{l}\text { ECTOR } \\
\text { A IN-MAX }\end{array}$ & ANG & REMARKS \\
\hline 61MII & 8381209 & $G, M U-T$ & $A B X$ & 6 & 22 & $D$ & $6-22$ & $N A I-D$ & & & \\
\hline $56 \mathrm{FU1}$ & 8381209 & $G, G$ & $A B X$ & 4 & 40 & $c$ & $4-40$ & $N A I-D$ & & 120 & \\
\hline 6ORE I & $83 B$ I 209 & $G, G$ & $A B X$ & 7 & & D & 7 & NAI -D & & DST & G-WDTH \\
\hline 6IMII & $83 B I 207$ & $G, G$ & $A B X$ & 9 & 22 & D & $9-22$ & NAI -D & & DST & \\
\hline 6ITOI & $83 B 1209$ & $G, G$ & $A B X$ & 3 & 16 & $c$ & $3-16$ & NAI I D & & 120 & \\
\hline $62 F U 4$ & $83 \mathrm{BI} 209$ & $G, G$ & $A B X$ & 4 & 9 & $c$ & $4-9$ & $N A I-D$ & & 120 & \\
\hline $63 \mathrm{~A} \times 1$ & $83 B I 209$ & $G, G$ & $A B X$ & 5 & 8 & D & $5-8$ & $N A I-D$ & & 135 & \\
\hline 63SU1 & 8381209 & $G, G$ & $A B X$ & 4 & 14 & $c$ & $4-14$ & NAI -D & & 120 & \\
\hline $63 Y 01$ & 8381209 & $G, G$ & $A B X$ & 6 & 8 & D & $6-8$ & $N A I-D$ & & DST & \\
\hline 64AR 1 & $83 \mathrm{BI} 209$ & $G, G$ & $A B X$ & 7 & & D & 7 & NAI $-D$ & & 135 & \\
\hline $61 C R 1$ & 8381209 & $E, E /$ & NOX & 0 & 5 & $\mathrm{Cl}$ & 83 & TEL-I & & DST & $J-P I, G-W D T H$ \\
\hline $63 B A I$ & 8381209 & $E, E, I$ & $S P C$ & 0 & 23 & D & 42 & $M A G-D$ & $19-42$ & 180 & $J-P I$ \\
\hline $56 \mathrm{FEI}$ & 8381209 & $G, N$ & $R L X$ & 7 & 20 & $c$ & $7-30$ & THR-D & $1-8$ & DST & \\
\hline $57 D E I$ & 8381209 & $G, N$ & RLY & 10 & 30 & $c$ & $10-30$ & $A C T-I$ & & 4PI & I SOMER \\
\hline $57 \mathrm{FEI}$ & 8381209 & $G, N$ & RLY & 10 & 30 & $c$ & 20,30 & THR - I & $5-$ & DST & \\
\hline $572 \mathrm{AI}$ & 8381209 & $G, N$ & SPC & 8 & 19 & c & 19 & EMU-D & $1-12$ & DST & REL YIELD \\
\hline $58 \mathrm{BE} 2$ & $83 B I 209$ & $G, N$ & $S P C$ & 8 & 16 & C & 14,16 & TOF $-D$ & $1-7$ & 120 & \\
\hline 60EMI & 8381209 & $G, N$ & SPC & 8 & 30 & C & 30 & EMU-D & $1-13$ & DST & \\
\hline $60 G E 3$ & 8381209 & $G, N$ & NOX & THR & & $\mathrm{CT}$ & $H R$ & BF 3-I & & 4PI & THRESHOLD \\
\hline $60 \mathrm{KU2}$ & $838 I 209$ & $G, N$ & RLY & 17 & 90 & $C$ & 90 & TEL-I & $10-$ & DST & \\
\hline 61TAI & 8381209 & $G, N$ & NOX & 13 & 22 & $c$ & 22 & THR - I & $6-$ & DST & \\
\hline 62805 & $83 B I 209$ & $G, N$ & $A B X$ & 8 & 22 & D & $8-22$ & NAI - I & & & \\
\hline $62 M I 3$ & $83 B$ I 209 & $G, N$ & $A B X$ & 8 & 22 & D & $8-22$ & $B F 3-I$ & & 4PI & \\
\hline 62 RE 1 & $83 B I 209$ & $G, N$ & NOX & 11 & 55 & $c$ & 55 & THR-I & 4- & DST & \\
\hline $632 A 1$ & $83 B I 209$ & $G, N$ & SPC & 8 & 19 & c & 14,19 & $E M U-D$ & $1-12$ & DST & \\
\hline $64 A L 4$ & $83 B 1209$ & $G, N$ & NOX & THR & 18 & $c$ & 18 & $S C I-I$ & 4- & DST & \\
\hline 64DE 1 & 8381209 & $\$ G, N$ & NOX & THR. & -80 & $c$ & 80 & $\mathrm{CCH}-\mathrm{D}$ & $0-16$ & 135 & NEUTRONS POLARIZED \\
\hline $64 G L 1$ & 8381209 & $G, N$ & SPC & 16 & & D & 16 & TOF -D & $0-5$ & 90 & \\
\hline 64HA2 & 8381209 & $G, N$ & $A B X$ & 6 & 27 & D & $6-26$ & $B F 3-I$ & & 4PI & \\
\hline $64 \mathrm{HAZ}$ & $83 B I 209$ & $G, 2 N$ & $A B X$ & 12 & 27 & D & $12-26$ & $B F 3-I$ & & $4 \mathrm{PI}$ & \\
\hline $56 G A 1$ & $83 B I 209$ & $G, \times N$ & $A B X$ & 7 & 27 & $c$ & $7-27$ & $B F 3-I$ & & $4 \mathrm{PI}$ & \\
\hline $56 \mathrm{HAl}$ & $83 B I 209$ & $G, \times N$ & $A B X$ & 15 & 18 & $D$ & 15,18 & $B F 3-I$ & & 4PI & \\
\hline $58 \mathrm{KAI}$ & 8381209 & $G, \times N$ & $A B X$ & 8 & 22 & c & $8-22$ & BF 3-I & & $4 \mathrm{PI}$ & \\
\hline $61 \mathrm{BA} 2$ & $83 B I 209$ & $G, \times N$ & $A B Y$ & 10 & 22 & c & 22 & THR-I & $3-$ & DST & \\
\hline 61BA2 & $83 B I 209$ & $G, \times N$ & $A B Y$ & 12 & 22 & $c$ & 22 & THR - I & $5-$ & DST & \\
\hline 61MII & $838 I 209$ & $G, \times N$ & $A B X$ & 6 & 22 & D & $6-22$ & BF 3-I & & $4 \mathrm{PI}$ & \\
\hline 61WA1 & 8381209 & $G, \times N$ & $S P C$ & 10 & 22 & C & 22 & $E M U-D$ & $3-9$ & DST & \\
\hline $62 \mathrm{FU} 4$ & $83 B I 209$ & $G, \times N$ & $A B X$ & 7 & 10 & C & $7-10$ & BF 3-I & & $4 \mathrm{PI}$ & \\
\hline $63 A^{2} 3$ & 8381209 & $G, X N$ & $A B Y$ & 9 & 85 & $c$ & 85 & $E M U-D$ & $1-12$ & DST & \\
\hline 61DE 2 & 8381209 & $G, F$ & $A B X$ & 300 & 999 & c 3 & $00-5$ & EMU-I & & $4 P I$ & \\
\hline
\end{tabular}


REF NUCLIDE REACTION RES EXCIT 58DU1 88RA
$G, F$
SOURCE DETECTOR ANG REMARKS MIN-MAX TYPE MIN-MAX

* yiELD-MASS DIST.

\begin{tabular}{|c|c|c|c|c|c|c|c|c|c|}
\hline A & ABUND. & & SE & AT ION & ENERG IES & (MEV) & & & \\
\hline 232 & 100.00 & $\begin{array}{l}G, N \\
6.4\end{array}$ & $\begin{array}{l}G, P \\
7.7\end{array}$ & $\begin{array}{c}G, T \\
10 .\end{array}$ & $\underset{*}{\mathrm{G}, \mathrm{HE} 3}$ & $\begin{array}{r}G, A \\
-4.1\end{array}$ & $\begin{array}{l}G, 2 N \\
11.4\end{array}$ & $\begin{array}{l}\text { G, NP } \\
14 .\end{array}$ & $\begin{array}{c}G, 2 P \\
*\end{array}$ \\
\hline
\end{tabular}

REF

NUCLIDE REACTION RES

EXCIT

IN, OUT

6ORE I

64LA 1

56 G I 1

$57 \mathrm{AV} 1$

$55 B A 4$

$56 G A 1$

$57 K A 1$

$61 B A 2$

$55 \mathrm{BA} 4$

$55 \mathrm{LA} 2$

$55 \mathrm{VAl}$

56DU1

$56 \mathrm{G} \mathrm{I} 1$

$56 \mathrm{KO} 2$

$56 \mathrm{KO} 3$

$57 \mathrm{AV} 1$

$57 \mathrm{BA} 4$

$575 C 2$

$58 \mathrm{FA} 2$

$60 \mathrm{KO} 6$

6OPR2

$61 \mathrm{BO} 2$

$61 D E 2$

62DE 3

$62 \mathrm{HU} 1$

$62 \mathrm{MO} 3$

$63 B A 3$

63DE 1

$63 \mathrm{KO} 1$

$62 \mathrm{MI} 1$
I OTH232

$90 \mathrm{TH} 232$

$90 \mathrm{TH} 232$

90 TH2 32

$90 \mathrm{TH} 232$

$90 \mathrm{TH} 232$

$90 \mathrm{TH} 232$

$90 \mathrm{TH} 232$

90 TH2 32

$90 \mathrm{TH} 232$

$90 \mathrm{TH} 232$

$90 \mathrm{TH} 232$

$90 \mathrm{TH}_{2} 32$

$90 \mathrm{TH} 232$

90 TH2 232

$90 \mathrm{TH} 232$

90 TH2 32

$90 \mathrm{TH} 232$

90 TH2 32

$90 \mathrm{TH} 232$

$90 \mathrm{TH} 232$

90 TH2 232

$90 \mathrm{TH} 232$

$90 \mathrm{TH} 232$

$90 \mathrm{TH} 232$

$90 \mathrm{TH} 232$

$90 \mathrm{TH} 232$

$90 \mathrm{TH} 232$

$90 \mathrm{TH} 232$

$90 \mathrm{TH} 232$
$G, G$

$$
G, G
$$

$A B X 7$

$A B X \quad 10 \quad 25$

$G, N$

$G, N$

$G, \times N$

$G, \times N$

$G, \times N$

$G, \times N$

$G, F$

$G, F$

$G, F$

$G, F$

$G, F$

$G, F$

$G, F$

$G, F$

$G, F$

$G, F$

$G, F$

$G, F$

$G, F$

$G, F$

$G, F$

$G, F$

$G, F$

$G, F$

$G, F$

$G, F$

$G, F$

$N, G$

RL I 7-13

ABY 12

$A B X$ THR -23

ABY THR 22

$A B Y 12$

$A B Y$

RLY

$\triangle B X$

THR -20
THR -10

SPC THR-70

$$
67
$$

$S P C$

ABX 6
$A B X \quad 8 \quad 16$

$A B X \quad 5 \quad 27$

ABY THR-19

ABY -820

ABX THR-24

SPC THR-18

RL I $\begin{array}{r}7-13 \\ T H R-20\end{array}$

THR-16

$A B \times 300999$ C300-999 EMU-I

$A B X \quad 7 \quad D \quad 7 \quad$ FMU I

ABY THR 20 C 12,20 BF 3-I

RLY 012

C 12

D 6

SPC THR 90 C 90

EMU-I

EMU-I

$\mathrm{ION}-\mathrm{D}$

$A C T-I$
$A B X \quad 6,7 \quad D \quad 6,7 \quad$ ION-I
ANG REMARKS

DST

DST

4P I

$4 P I$

* rel to g,f

$4 P I$

DST

4PI

* delayed neutrons

* av. nO. NEUTRONS

* symmetric fiss.

4P I

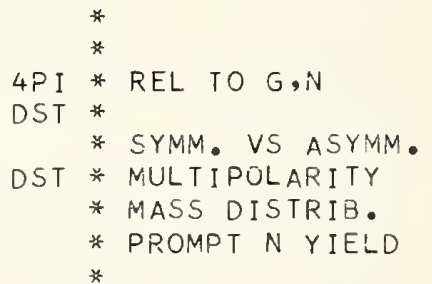

$4 P I$

DST

0

4PI DELAYED N YIELDS

DST

DST

DST

4PI 


\begin{tabular}{|c|c|c|c|c|c|c|c|c|c|}
\hline A & ABUND. & & SE & AT I ON & ENERGIES & $(M E V)$ & & & \\
\hline & & $\mathrm{G}, \mathrm{N}$ & $G, P$ & $G, T$ & $\mathrm{G}, \mathrm{HE} 3$ & $G, A$ & $G, 2 N$ & $G, N P$ & $\begin{array}{l}G, 2 P \\
12.0\end{array}$ \\
\hline 34 & $5.6(-3)$ & 6.8 & 6.6 & 10.3 & 10.6 & $-4 \cdot 8$ & 12.7 & 13.3 & 12.0 \\
\hline $\begin{array}{l}35 \\
38\end{array}$ & $\begin{array}{r}0.72 \\
99.27\end{array}$ & $\begin{array}{l}5.3 \\
6.1\end{array}$ & $\begin{array}{l}6.7 \\
7.6\end{array}$ & $\begin{array}{l}10.0 \\
10.0\end{array}$ & $\begin{array}{l}9.5 \\
*\end{array}$ & $\begin{array}{l}-4 \cdot 7 \\
-4.3\end{array}$ & $\begin{array}{l}12.0 \\
11.4\end{array}$ & 11.9 & $\begin{array}{l}2 \cdot 3 \\
*\end{array}$ \\
\hline
\end{tabular}

\begin{tabular}{|c|c|c|c|c|c|c|c|c|c|c|c|c|}
\hline REF & $\begin{array}{l}\text { NUCI } \\
Z\end{array}$ & $\begin{array}{r}\text { IDE } \\
A\end{array}$ & $\begin{array}{l}\text { REACT ION } \\
\text { IN, OUT }\end{array}$ & RES & $E \times C$ & EIT & & $\begin{array}{l}\text { OURCE } \\
\text { IIN-MAX }\end{array}$ & $\begin{array}{l}\text { DET } \\
\text { TYPE }\end{array}$ & $\begin{array}{l}\text { CTOR } \\
\text { IN-MAXX }\end{array}$ & ANG & REMARKS \\
\hline $58 \mathrm{MO} 2$ & $92 U$ & & G, MU-T & $A B X$ & THR & 94 & $c$ & 94 & $S C I-D$ & & $4 \mathrm{PI}$ & * \\
\hline 56 FUI & $92 \mathrm{U}$ & & $G, G$ & $A E X$ & 4 & 40 & $c$ & $4-40$ & NAI $-D$ & & 120 & \\
\hline $58 \mathrm{MOI}$ & 920 & & $G, G$ & $A B X$ & 90 & & & 90 & & & DST & * SMALL ANGLES \\
\hline $63 \mathrm{BO} 2$ & $92 \mathrm{U}$ & & $G, G$ & $A B X$ & 9 & & $D$ & 9 & NAI $-D$ & & DST & \\
\hline 6ITAl & $92 \mathrm{U}$ & & $G, \stackrel{\dot{N}}{ }$ & NOX & THR & 22 & $c$ & 22 & THR-I & $6-$ & DST & \\
\hline $61 B A 2$ & $92 U$ & & $G, \times N$ & $A B Y$ & THR & 22 & $c$ & 22 & THR - I & $5-$ & DST & \\
\hline $64 \mathrm{GL} 1$ & $92 \mathrm{U}$ & & $G, N$ & $S P C$ & 16 & & D & 16 & TOF $-D$ & $0-5$ & 90 & \\
\hline $55 \mathrm{BA}_{4}$ & $92 \mathrm{U}$ & & $G, \times N$ & $A B Y$ & 12 & & c & 19 & BF 3-I & & $4 \mathrm{PI}$ & \\
\hline $56 G A 1$ & $92 U$ & & $G, \times N$ & $A B X$ & 5 & 27 & $c$ & $5-27$ & $B F 3-I$ & & 4P I & \\
\hline $55 B A 4$ & 920 & & $G, F$ & $A B Y$ & 12 & & $c$ & 19 & I ON-I & & 4P I & \\
\hline $55 \mathrm{KAl}$ & $92 U$ & & $G, F$ & $A B X$ & & & $c$ & & & & & * SYMM. VS ASYMM. \\
\hline $55 \mathrm{LA} 2$ & $92 \mathrm{U}$ & & $G, F$ & $A B Y$ & & & & & & & & * DELAYED NEUTRON \\
\hline $55 \mathrm{VAl}$ & $92 \mathrm{U}$ & & $G, F$ & $A B Y$ & THR- & -19 & $c$ & 19 & ION-I & & & * AV. NO. NEUTRC \\
\hline $56 \mathrm{BA} 1$ & $92 \mathrm{U}$ & & $G, F$ & & THR- & -27 & c & $9-27$ & FMU - I & & DST & * \\
\hline 56001 & $92 \mathrm{U}$ & & $G, F$ & $R L Y$ & & & c & $5-$ & & & & * SYMMETRIC FISS \\
\hline $56 \mathrm{KO} 2$ & $92 U$ & & $G, F$ & $A B X$ & THR- & -24 & $c$ & $8-24$ & & & & * \\
\hline $56 \mathrm{k03}$ & $92 \mathrm{U}$ & & $G, F$ & $S P C$ & THR- & -18 & $c$ & 18 & I ON-D & & & * \\
\hline $57 B A 4$ & $92 \mathrm{U}$ & & $G, F$ & & THR- & -27 & c & $9-27$ & & & DST & it \\
\hline $575 C 2$ & $92 U$ & & $G, F$ & & THR- & -10 & $c$ & $4-10$ & & & & * SYMM. VS ASYMi \\
\hline $58 B A 7$ & 920 & & $G, F$ & & THR - & -27 & c & $6-27$ & & & DST & * \\
\hline 60FOI & 920 & & $G, F$ & NOX & & 20 & c & $6-20$ & EMU - I & & DST & \\
\hline $60 \mathrm{KO} 6$ & 920 & & $G, F$ & SPC & THR- & -70 & $c$ & 70 & & & & * NIASS DISTRIB. \\
\hline 60PR2 & 920 & & $G, F$ & & 6 & 7 & D & $6-7$ & & & & * PROMPT $N$ YIELD \\
\hline 6OTA3 & 920 & & $G, F$ & $S P C$ & 6 & & D & 6 & $E M U-D$ & & DST & \\
\hline $610 E 1$ & $92 \mathrm{U}$ & & $G, F$ & NOX & 6 & 20 & c & $6-20$ & EMU-I & & DST & \\
\hline 61DE2 & $92 \mathrm{U}$ & & $G, F$ & $A B X$ & 300 & 999 & & $300-990$ & EMU-I & & $4 \mathrm{PI}$ & \\
\hline $62 B 08$ & $92 U$ & & $G, F$ & $S P C$ & THR & 50 & c & $17-50$ & ION-D & & 90 & \\
\hline $62 \mathrm{KO}$ & $92 \mathrm{U}$ & & $G, F$ & NOX & THR & 35 & $c$ & 35 & ION-D & & DST & MASS DISTRIBUTION \\
\hline $62 \mathrm{MO} 3$ & $92 U$ & & $G, F$ & $A B Y$ & THR & 20 & $c$ & 12,20 & BF 3-I & & 4PI & DELAYED N YIELDS \\
\hline $63 \mathrm{BO} 06$ & $92 \mathrm{U}$ & & $G, F$ & NOX & THR & 35 & $c$ & 35 & $I O N-O$ & & DST & \\
\hline 63PE2 & $92 \mathrm{U}$ & & $G, F$ & RLY & THR & 14 & c & 14 & $A C T-I$ & & 4P I & \\
\hline $57 \mathrm{KAl}$ & $92 \mathrm{U}$ & 233 & $G, \times N$ & $A B X$ & THR - & -23 & $c$ & -23 & & & & * \\
\hline $57 \mathrm{BA} 4$ & $92 U$ & 233 & $G, F$ & & THR- & -20 & c & $6-20$ & & & DST & * \\
\hline $62 \mathrm{HUI}$ & 920 & 233 & $G, F$ & $A B X$ & 6 , & & D & 6,7 & $I O N-I$ & & 0 & \\
\hline $57 B A 4$ & $92 \mathrm{U}$ & 234 & $G, F$ & & THR - & -20 & $c$ & $6-20$ & & & DST & * \\
\hline $62 \mathrm{HUI}$ & 920 & 234 & $G, F$ & $A B X$ & 6 , & & D & 6,7 & $I O N-I$ & & 0 & \\
\hline $64 \mathrm{BO} 3$ & $92 U$ & 235 & $G, N$ & $A B X$ & & -21 & D & $8-21$ & $B F 3-I$ & & 4PI & \\
\hline $64 \mathrm{BO} 3$ & $92 \mathrm{U}$ & 235 & $G, 2 N$ & $A B X$ & & -21 & D & $8-21$ & $B F 3-I$ & & 4PI & \\
\hline $57 \mathrm{BA}_{4}$ & $92 \mathrm{U}$ & 235 & $G, F$ & & THR- & -20 & c & $6-20$ & & & DST & * \\
\hline $62 \mathrm{HUI}$ & $92 \mathrm{U}$ & 235 & $G, F$ & $A B X$ & 6 , & 7 & D & 6,7 & $I O N-I$ & & 0 & \\
\hline $64 \mathrm{BO} 3$ & 920 & 235 & $G, F$ & $A B X$ & & -19 & D & $6-19$ & ION-I & & 4PI & \\
\hline $57 B A 4$ & 920 & 236 & $G, F$ & & THR- & -20 & $c$ & $6-20$ & & & DST & * \\
\hline $62 \mathrm{HUI}$ & $92 \mathrm{U}$ & 236 & $G, F$ & $A B X$ & 6 , & & D & 6,7 & $I O N-I$ & & 0 & \\
\hline $56 G 11$ & $92 U$ & 238 & $G, N$ & $A B X$ & 8 & 16 & $c$ & $8-20$ & $A C T-I$ & & $4 \mathrm{PI}$ & \\
\hline $57 \mathrm{KAl}$ & 920 & 238 & $G, \times N$ & $A B X$ & THR- & -23 & $c$ & -23 & & & & * \\
\hline $56 \mathrm{GI} \mathrm{I}$ & $92 \mathrm{U}$ & 238 & $G, F$ & $A B X$ & 8 & 20 & $c$ & $8-20$ & $A C T-I$ & & 4P I & \\
\hline
\end{tabular}




\begin{tabular}{|c|c|c|c|c|c|c|c|c|c|c|}
\hline PEF & $\underset{Z}{N U C}$ & $\begin{array}{r}I D E \\
A\end{array}$ & $\begin{array}{l}\text { REACTION } \\
\text { IN,OUT }\end{array}$ & RES & EXCIT & & $\begin{array}{l}\text { OURCE } \\
\text { IN-MAX }\end{array}$ & $\begin{array}{c}\text { DETECTOR } \\
\text { TYPE MIN-MAX }\end{array}$ & ANG & REMARKS \\
\hline $57 B A 4$ & $92 \mathrm{U}$ & 238 & $G, F$ & & THR -20 & C & $6-20$ & & $D S T$ & * \\
\hline 60801 & $92 \mathrm{U}$ & 238 & $G, F$ & SPC & THR 70 & C & 70 & I ON-D & & \\
\hline 62 DE 3 & $92 \mathrm{U}$ & 238 & $G, F$ & $A B X$ & 7 & D & 7 & EMU-I & DST & \\
\hline $62 \mathrm{HU} 1$ & $92 \mathrm{U}$ & 238 & $G, F$ & $A B X$ & 6,7 & D & 6,7 & $I O N-I$ & 0 & \\
\hline $63 D^{1} 1$ & $92 \mathrm{U}$ & 238 & $G, F$ & $A B X$ & 7 & D & 7 & EMU-I & DST & \\
\hline
\end{tabular}

NEPTUNIUM $Z=93$

\begin{tabular}{|c|c|c|c|c|c|c|c|c|c|}
\hline \multirow[t]{2}{*}{ REF } & NUCL IDE & REACTION & RES & EXCIT & \multirow{2}{*}{\multicolumn{2}{|c|}{$\begin{array}{l}\text { SOURCE } \\
\text { MIN-MAX }\end{array}$}} & DETECTOR & \multirow[t]{2}{*}{ ANG } & \multirow[t]{2}{*}{ REMARKS } \\
\hline & $z$ & IN,OUT & & & & & TYPE MIN-MAX & & \\
\hline A4 & 93 NP 237 & $G, F$ & & $T H R-20$ & $C$ & $6-20$ & & DST & * \\
\hline U1 & $93 N P 237$ & $G, F$ & $A B X$ & 6,7 & 0 & 6,7 & $1 O N-1$ & 0 & \\
\hline
\end{tabular}

\begin{tabular}{|c|c|c|c|c|c|c|c|c|c|}
\hline REF & NUCL IDE & REACTION & RES & $\mathrm{EXCIT}$ & \multirow{2}{*}{\multicolumn{2}{|c|}{$\begin{array}{l}\text { SOURCE } \\
\text { MIN-MAX }\end{array}$}} & DETECTOR & \multirow[t]{2}{*}{ ANG } & \multirow[t]{2}{*}{ REMARKS } \\
\hline & $z$ & IN,OUT & & & & & TYPE MIN-MAX & & \\
\hline $57 K A 1$ & $94 P \cup 239$ & $G, \times N$ & $A B X$ & THR -23 & $C$ & -23 & & & * \\
\hline $57 B A 4$ & $94 P \cup 239$ & $G, F$ & & $T H R-20$ & c & $6-20$ & & DST & * \\
\hline $7 B A 4$ & $94 P \cup 240$ & $G, F$ & & THR -20 & C & $6-20$ & & DST & * \\
\hline
\end{tabular}

$\begin{array}{ccllll}\text { REF NUCLIDE REACTION RES EXCIT SOURCE } & \text { DETECTOR ANG REMARKS } \\ & Z & \text { A IN,OUT } & & \text { MIN-MAX TYPE MIN-MAX } & \end{array}$ $57 B A 495 A M 241$ G, F THR-20 C 6-20 
PICNS refers to Proceedings of the International Conference on Nuclear Structure, University of Toronto Press, 1960

55 Al 1 L. Allen, Jr。, Phys。 Rev• 98, 705 (1955)

$55 \mathrm{Ba} 1$ Wo Co Barber, Wo Do George, Do Do Reagan, Phys。 Rev。 98, 73 (1955)

$55 \mathrm{Ba} 2 \mathrm{R}$ 。 Basile, Co Schuhl, Compt。 Rend。240, 2399 (1955)

$55 \mathrm{Ba} 3$ R。 Basile, C。 Schuhl, Wo Sebaoun, Compt。 Rend。 241,387 (1955)

$55 \mathrm{Ba} 4$ B。 No Baluev, B. I. Gavrilov, Go No Zatsepina, L. E。 Lazareva; Zhur。Eksp。 i Teoret。Fiz。 29, 280 (1955); Soviet Phys。 JETP 2, 106 (1956)

$55 \mathrm{Ba} 5$ P. S. Baranov, V. I. Gol'danskii; Zhur. Eksn. i Teoret. Fiz. 28, 746, (1955); Soviet Phys。 JETP 1, 613 (1955)

55 Bo 1 O. A. Borello, Jo Goldemberg, M. D. S。 Santos; An。 Acad。 Brasil. Cienc. 27, 413 (1955)

55 Bo 2 O. A. Borello, Jo Goldemberg, M. D. S. Santos; An. Acad. Brasil. Cienc. 27, 417 (1955)

$55 \mathrm{Bu} 1$ J. Lo Burkhardt; Phys。 Rev. 100, 192 (1955)

$55 \mathrm{Bu} 2$ J。 L。 Burkhardt, E。 JoWinhold, To Ho Dupree; Phys. Rev. 100, 199-202(1955)

$55 \mathrm{Ca} 1 \mathrm{~J}$. Go Campbel1, Australian J。 Phys。 8, 449 (1955)

$55 \mathrm{Ca} 2$ J。 H。 Carver, H。 J० Hay, E。 Wo Titterton; Phil。 Mag. 46,841 (1955)

$55 \mathrm{Ch} 1$ B. G。 Chidley, L. Katz; Phys. Rev. 99, 1646 (1955)

55 Co 1 R. D. Cooper, D. J. Zaffarano; Phys. Rev• 98, 1199 (1955)

$55 \mathrm{Da} 1$ W. K. Dawson; Phys. Rev. 99, 1648 (1955)

55 De 1 M. D. DeSouza Santos, J。 Goldemberg, R。 R。 Pieroni, E. Silva, O. A. Borello, So So Villaca, J。 Lo Lopes; Int. Conf. Peaceful Uses of Atomic Energy II (United Nations, N. Y。, 1955), 169

55 Di 1 W. R. Dixon, Can。 J。 Phys。 33, 785 (1955)

55 Dl 1 Z. Dlouhy, V。 Petrzilka, M० Rozkos; Czech。J。 Phys. 5, 193 (1955)

55 Er 1 P. Erdos, Po Jordan, Po Stoll; Helv。 Phys。 Acta 28, $322(1955)$

55 Gl 1 Ho Glattli, E。 Loepfe, Po Stoll; Helv。 Phys。Acta $28,366(1955)$

55 Go 1 F。 K. Goward, J० J० Wilkins; Proc॰Roy॰ Soc。 (London) A228, 376 (1955)

55 Ha 1 F. I. Havlicek, B. Dobovisek; Phys。Rev。 100, 1355 (1955)

$55 \mathrm{He} 1$ F. Heinrich, R。 Rubin; Helva. Phys。Acta 28, 185 (1955)

55 Jo 1 S。 A. E。 Johansson; Phys. Rev• 97, 434 (1955)

$55 \mathrm{Ka} 1$ L。 Katz, T. M. Kavanagh, A. G。 W。 Cameron, E。 C. Bailey, J. W. T. Spinks; Phys。 Rev。 99, 98 (1955)

55 La 1 W. B. Lasich, E. G. Muirhead, G。 Go Shute; Australian J. Phys. 8, 456 (1955) 
55 La 2 L. E. Lazareva, B. S. Ratner, I. V. Shtranikh;

Zhur. Eksp. i Teoret. Fiz. 29, 274 (1955); Soviet Phys. JETP 2, 201 (1956)

55 Li 1 D. L. Livesey, Can. J. Phys. 33, 391 (1955)

55 Mc 1 K. G. McNeill; Phil. Mag. 46, 321 (1955)

55 Me 1 F. R. Metzger, Phys. Rev. 98, 200 (1955)

55 Mi 1

M. Miwa; J. Phys。 Soc. Japan 10, 173 (1955)

$55 \mathrm{Na} 1$

R. Nathans, P. F. Yergin, Phys. Rev. 98, 1296 (1955)

55 Od 1 A. C. Odian, W. Rankin, P. C. Stein, A. Wattenburg; Phys. Rev. 98, 248 (1955)

55 Ox 1 C. L. Oxley, V。 Lo Telegdi; Phys. Rev. 100, 435 ( 1955)

$55 \mathrm{Pe} 1 \mathrm{~A} . \mathrm{S}$. Penfold, B. Mo Spicer, Phys。Rev. 100, 1377 (1955)

$55 \mathrm{Pe} 2 \mathrm{~J}$. E. Perry, S. J. Bame, Jr.; Phys. Rev. 99, 1368( 1955)

55 Re 1 Do Reagan, Phys. Rev。 100, 113 (1955)

$55 \mathrm{Ri} 1$ L. S。 Ring, Jr.; Phys。 Rev。 99, 137 (1955)

$55 \mathrm{Sa} 2$ D. Sadeh; Compt. Rend. 249, 2313 (1959)

55 Sc 1 M. B. Scott, A. O. Hanson, D. W. Kerst; Phys. Rev. 100,209 (1955)

55 Sc 2 J. Schmouker, Po Erdos, P. Jordan, Po Stoll; J. Phys. Radium 16, 169 (1955)

$55 \mathrm{Sp} 1$ B. M. Spicer, Phys. Rev。99, 33 (1955)

$55 \mathrm{Sp} 2$ B. M. Spicer, A. S. Penfold; Phys。Rev. 100, 1375 (1955)

$55 \mathrm{Sp} 3$ B. Mo Spicer; Phys. Rev。 100, 791 (1955)

55 St 1 W. E. Stephens, A. K. Mann, B. J. Patton, E. J. Winhold; Phys。 Rev。 98, 839 (1955)

55 St 2 W. E. Stephens, A. K. Mann; Phys. Rev. 98, 241 (1955)

$55 \mathrm{Ta} 1$ C. A. Tatro, T。R。 Palfrey, Jr。; Phys. Rev. 100, $1265(1955)$

55 To 1 M. E. Toms, W. E. Stephens; Phys. Rev. 98, 626 (1955)

$55 \mathrm{Va} 1$ B. N. Valuev, B. I. Gavrilov, G. N. Zatsepina, L. E. Lazareva; Zhur. Eksp. i Teoret. Fiz. 29, 280 (1955); Soviet Phys. JETP 2, 106 (1956)

55 Vi 1 S. S. Villaca, J. Goldemberg; An. Acad. Brasil. Cienc. 27, $427(1955)$

55 Ye 1 Po Fo Yergin, B. P. Fabricand; Phys。 Rev • 100, 1249 (1955)

56 At 1 J. R. Atkinson, I. Crawford, D. R. Morrison, I. Preston,

I. F. Wright; Physica 22, 1145 (1956)

56 Ax 1 Po Axel, J. D. Fox; Phys. Rev. 102, 400 (1956)

$56 \mathrm{Ba} 1$ B. P. Bannik, K. M. Kulikova, L. E. Lazareva, V. A. Yakovlev; Physica 22, 1186 (1956)

56 Bo 1 O. V. Bogdankevich, L. E. Lazareva, F. A. Nikolaev; Zhur. Eksp. i Teoret. Fiz. 31, 405 (1956); Soviet Phys. JETP 4, 320 (1957)

$56 \mathrm{Ch} 1$ R. Chastel, Compt. Rend 242, 1440 (1956); Compt. Rend 242, 2337 (1956); J. Phys。Rad. 17, 518 (1956)

56 Co 1 L. Cohen, A. K. Mann, B. J. Patton, K. Reibel, W. E. Stephens, E. Jo Winhold; Phys。 Rev. 104, 108 (1956)

$56 \mathrm{Da} 1$ W. K. Dawson, D. L. Livesey; Can。 J. Phys。 34, 241 ( 1956 )

$56 \mathrm{Da} 2$ W. K. Dawson; Can. J. Phys. 34, 1480 (1956)

56 Di 1 D. R. Dixon, K. C. Bandtel; Phys. Rev. 104, 1730 (1956)

$56 \mathrm{Du} 1$ R. B. Duffield, R。 A. Schmitt; Physica 22, 1186 (1956) 
56 Ed 1 R. D. Edge, Nuclear Phys. 2, 485 (1956)

56 Ed 2 R. D. Edge, Australian J. Phys. 9, 429 (1956)

$56 \operatorname{Er} 1$ Jo Ero, L. Keszthelyi, Nuclear Phys. 2, 371 (1956)

56 Fa 1 B. P. Fabricand, B. A. Allison, J. Halpern; Phys. Rev . $103,1755(1956)$

56 Fe 1 F. Ferrero, A. O. Hanson, R. Malvano, C. Tribuno; Nuovo Cimento 4, 418 (1956)

56 Fo 1 Bo Forkman; Arkiv Fysik 11, 265-275 (1956)

$56 \mathrm{Fr} 1$ Jo Ho Fregeau; Physo Rev. 104, 225 (1956)

56 Fu 1 E. G。 Fuller, E。 Hayward; Phys. Rev• 101, 692 (1956)

$56 \mathrm{Ga} 1$ B. I. Gavrilov, L. E. Lazareva; Zhur. Eksp。 i Teoret. Fiz. 30, 855 (1956); Soviet Phys。 JETP 3, 871 (1957)

$56 \mathrm{Gi} 1 \mathrm{~J}$. E. Gindler, J. R. Huizenga; Phys。 Rev。104, 425 (1956)

56 Ha 1 W. H. Hartley, W. E. Stephens, E. J. Winhold; Phys.

Rev. $104,178(1956)$

56 He 1 F. Heinrich, H. Waffler; Helv. Phys. Acta 29, 232 (1956)

$56 \mathrm{He} 2 \mathrm{~F}$. Heinrich, H. Waffler, M. Walter; Helv. Phys. Acta 29, $3(1956)$

56 Jo 1 S. A. E. Johansson; Physica 22, 1144 (1956)

$56 \mathrm{Ke} 1$ J. C. Keck, A. V. Tollestron, Phys. Rev. 101, 360 (1956)

$56 \mathrm{Kl} 1$ G. K. Kliger, V. I. Ryabinkin, I. V. Chuvilo, V. G. Shevchenko; Dhysica 22, 1142 (1956)

56 Ko 1 A. P. Komar, I. D. Iavor, Zhur. Eksp. i Teoret. Fiz. 31 531 (1956); Soviet Dhys. JETP 4, 432 (1957)

56 Ko 2 V. A. Korotkova, P. A. Cherenkov, I. V. Chuvilo; Dokl. Akad. Nauk SSSR 106, 633 (1956); Soviet Phys. Doklady 1, $77(1956)$

56 Ko 3 V. A. Korotkova, P. A. Cherenkov, I. V. Chuvilo; Dokl. Akad. Nauk SSSR 106, 811 (1956); Soviet Dhys. Doklady 1, $104(1956)$

56 Le 1 E. Lejkin, R. Osokina, B. Ratner; Nuovo Cimento 3, Suppl. 1, 105 (1956)

56 Li 1 D. L. Livesey, Can. J. Phys. 34, 216 (1956)

56 Li 2 D. L. Livesey, Can. J. Phys. 34, 1022 (1956)

56 Ma 1 A. K. Mann, E. W. Titterton, Proc. Phys. Soc. 69, $917(1956)$

56 Me 1 F. R. Metzger, Phys. Rev. 101, 286 (1956)

56 Me 2 F. R. Metzger, Phys. Rev. 103, 983 (1956)

56 Me 3 L. Meyer Schutzmeister, V。 L. Telegdi; Phys. Rev。 104, $185(1956)$

56 Od 1 A. C. Odian, P. C. Stein, A. Wattenberg, B. T. Feld,

R. Weinstein; Dhys. Rev. 102, 837 (1956)

56 Os 1 R. M. Osokina, B. S. Ratner; Physica 22, 1147 (1956)

56 Pr 1 J. D. Prentice, K. G. McNeill, Phil. Mag. 1, 373 (1956)

56 Re 1 J. M. Reid, P. Swinbank, J. R. Atkinson; Physica 22 , $1142(1956)$

56 Sc 1 C. Schuh1, R. Basile; Physica 22, 1144 (1956)

56 Si 1 E. Silva, J. Goldemberg; Nuovo Cimento 3, 12 (1956)

56 Si 2 E. Silva, J. Goldemberg; An. Acad. Brasil. Cienc. 28, $275(1956)$

56 St 1 W. E. Stephens; Physica 22, 1143 (1956)

$56 \mathrm{Su} 1$ T. T. Sugihara, I. Halnern; Phys. Rev. 101, 1768 (1956) 
$56 \mathrm{Tz} 1$ C. Tzara; Compt。Rend。242, 2340 (1956)

56 Tz 2 C. Tzara; J. Phys。 Radiun 17, 1001 (1956)

56 Wa 1 Ho Waffler, F. Heinrich; Physica 22, 1146 (1956)

56 Wh 1 E. A. Whalin, B. D. Schriever, A. O. Hanson; Phys. Rev. 101,377 (1956)

56 Wo 1 R. L。 Wolke, N.A. Bonner; Phys。Rev. 102, 530 ( 1956 )

56 Wr 1 I. F。 Wright, D. R。 O. Morrison, J。M。Reid, J. R。

Atkinson; Proc. Phys。 Soc. A 69, 77 (1956)

56 Ye 1 P. F。 Yergin, Phys。 Rev。104, 1340 (1956)

56 Ye 2 P. F。 Yergin, B。 Po Fabricand, Phys. Rev• 104, 1334 (1956)

57 Al 1 Yu。 A. Aleksandrov, N. B. Delone, L. I. Slovokhotov,

G. A. Sokol, L。 N. Shtarkov; Zhur. Eksp。 i Teoret. Fiz。

33, 614 (1957); Soviet Phys. JETP 6, 472 (1958)

57 Av 1 No Po Avdonin, Ko A. Petrzhak; Trud。 Leningr. Tekhnol。

Inst. Im。 Lensoveta 40, 5 (1957)

$57 \mathrm{Ba} 1$ P. S. Baranov, V. I. Gol 'danskii, V. S. Roganov; Zhur. Eksp. i Teoret。Fiz。33, 1123 (1957); Soviet Phys。 JETP 6, 865 (1958); Phys。Rev。109, 1801 (1958)

$57 \mathrm{Ba} 2$ E. B. Bazhanov, Yu. M. Volkov, A. Po Komar, L. A. Kul'chitskii, V. P. Chizhov; Dokl。Akad。 Nauk SSSR 113, 65 (1957): Soviet Phys。 Doklady 2, 107 (1957)

$57 \mathrm{Ba} 3$ R。 Basile; Ann。 Phys. (Paris) 2, 267 (1957)

$57 \mathrm{Ba} 4$ B. Po Bannik, No Mo Kulikova, Lo E. Lazareva, V. A. Yakovlev; Zhur。Eksp。i Teoret。Fiz. 33, 53 (1957); Soviet Phys。 JETP $6,39(1958)$

57 Be 1 Jo B. Bellicard, J。 Miller, C。 Tzara; J。 Phys. Radium 18, 201 (1957)

57 Bo 1 F. Bobard, G. Boulegue, P. Chanson; Compt. Rend. 244, 1761 (1957)

$57 \mathrm{Br} 1$ P. Brix, E。 K。 Maschke, Z。 Naturf̂orsch. 12, 1013 (1957)

$57 \mathrm{Ca} 1$ Jo Ho Carver, R。 D. Edge, K. H. Lokan; Proc. Phys. Soc. A $70,415(1957)$

$57 \mathrm{Ca} 2$ Jo Ho Carver, K. H. Lokan; Australian Jo Phys. 10, $312(1957)$

$57 \mathrm{Ch} 1$ I. V. Chuvilo, V. G. Shevchenko; Zhur. Eksp. i Teoret. Fiz. 32, 1335 (1957); Soviet Phys. JETP 5, 1090 (1957)

57 Co 1 B. C. Cook, Phys。Rev。106, 300 (1957)

$57 \mathrm{De} 1$ So DeBenedetti, U. Farinelli, F。 Ferrero, R. Malvano,

G. Pelli, C. Tribuno; Nuovo Cimento 6, 682 (1957)

57 El 1 A. El Sioufi, P. Erdos, P. Stoll; Helv. Phys. Acta 30,264 (1957)

57 El 2 A. El Sioufi, P. Erdos, Po Stoll; Helv。 Phys. Acta 30 266 (1957)

$57 \operatorname{Er} 1$ Po Erdos, P. Scherrer, Po Stoll; Helva. Phys. Acta $30,639(1957)$

$57 \mathrm{Fe} 1$ F。 Ferrero, L。 Gonella, R. Malvano, C. Tribuno, A. O。 Hanson; Nuovo Cimento 5, 242 (1957)

$57 \mathrm{Fe} 2 \mathrm{~F}$. Ferrero, R. Malvano, C. Tribuno; Nuovo Cimento $6,385(1957)$

$57 \mathrm{Fe} 3$ F。 Ferrero, A。 O. Hanson, R。 Malvano, C。Tribuno; Nuovo Cimento $6,585(1957)$ 
57 Go 1 A. N. Gorbunov, V. M. Spiridonov, Zhur. Exsp. i Teoret.

Fiz. 33, 21 (1957); Soviet Phys. JETP 6, 16 (1958)

57 Ia 1 E. Ilayward, E. G. Fuller; Phys. Rev. 106, 991 (1957)

57 He 1 R. M. Henry, D. S. Martin, Jr॰; Phys. Rev. 107, 772 (1957)

$57 \mathrm{Hi} 1$ R. L. Hines, Dhys. Rev. 105, 1534 (1957)

57 Jo 1 S. A. E. Johansson, B. Forkman; Arkiv Fysik 12, 359 (1957)

$57 \mathrm{Ka} 1$ L. Katz, K. G. McNei1l, M. LeBlanc, F. Brown; Can. J. Phys. $35,470(1957)$

57 Ki 1 A. No King, A. F. Voigt; Dhys. Rev. 105, 1310 (1957)

$57 \mathrm{Kn} 1$ V. Knapn; Proc. Phys. Soc。 (London) A70, 142 (1957)

57 Ko 1 A. Po Komar, I。 Po Iavor; Zhur॰ Eksp。 i Teoret。 Fiz。

32,614 (1957); Soviet Phys. JETP 5, 508 (1957)

57 Ko 2 A. Po Komar, I。 P. Yavor; Zhur。Eksp. i Teoret. Fiz。 31, 531 (1957); Soviet Phys。 JETP 4, 432 (1957)

57 Li 1

D. L. Livesey; Can. J。 Phys。 35, 987 (1957)

57 Lo 1

K. H. Lokan, Proc. Phys. Soc. A 70, 836 (1957)

$57 \mathrm{Mi} 1$

C. Milone, R. Ricamo, Nuovo Cimento 5, 1338 (1957)

57 Mi 2

C. Milone, R. Ricamo, A. Rubbino; Nuovo Cimento 5,528(1957)

57 Mi 3

C. Milone, R. Ricamo, R。 Rinzivillo; Nuovo Cimento 5, $532(1957)$

$57 \mathrm{Mu} 1 \mathrm{~J}$. Muto, E。 Takekoshi, T。 Nakamura, A。 Imamura,

Y. Tsuneoka; J. Phys. Soc. Japan 12, 109 (1957)

57 Ro 1 M. Rozkos; Czech。 Jo Phys。 7, 592 (1957)

57 Ro 2 M. Rozkos; Czech。 J. Phys。 7, 20 (1957)

57 Sc 1 F。 D。 Schupp, C. B. Colvin, D. S。 Martin, Jr•;

Phys. Rev. 107, 1058 (1957)

57 Sc 2 R. A. Schmitt, R。 B。 Duffield; Phys。 Rev。 105, 1277 (1957)

$57 \mathrm{Sp} 1$ B. M. Spicer, E. Go Muirhead, G. Go Shute; Australian

J. Phys. 10, 217 (1957)

$57 \mathrm{Sp} 2$ Bo Ho Spicer, Australian Jo Phys. 10, 326 (1957)

$57 \mathrm{~Sv} 1$ No Lo Svantesson, Nuclear Phys。 3, 273 (1957)

57 Sw 1 C. P. Swann, F. R. Metzger, Phys。Rev• 108, 982 (1957)

57 To 1 M. E. Toms, W. E. Stephens, Phys. Rev. 108, 77 (1957)

$57 \mathrm{Za} 1$ G. N. Zatsepina, L。 E. Lazareva; Zhur。 Eksp。 i Teoret。 Fiz. 32,27 (1957); Soviet Phys。 JETP 5, 21 (1957)

58 Al 1 L。 W. Alvarez, F. So Crawford, Jr॰, M. L. Stevenson; Phys. Rev. 112, 1267 (1958)

58 As 1 T. Asada, M. Masuda, Mo Okumura, J。 Okuma; J。 Phys。 Soc. Japan 13, 1 (1958)

$58 \mathrm{Au} 1$ L. B. Aul1, W. D。 Whitehead; Phys. Rev。110, 1113 (1958)

$58 \mathrm{Ba} 1$ P. S。 Baranov, V。 I. Golidanskii, V. S. Roganov; Phys。 Rev。109, 1801 (1958)

$58 \mathrm{Ba} 2$ W. Co Barber; Phys. Rev. 111, 1642 (1958)

$58 \mathrm{Ba} 3$ M. Q. Barton, J. H. Smith; Phys。Rev• 110, 1143 (1958)

$58 \mathrm{Ba} 4$ W. C. Barber, W. R. Dodge, V. J. Vanhuyse; Proceedings of the International Conference on Nuclear Physics(1958), 630

$58 \mathrm{Ba} 5$ R. Baisle, M. Gusakow; Compt. Rend. 246, 588 (1958)

$58 \mathrm{Ba} 6$ E. B. Bazhanov, Yu. M. Volkov, L. A. Kul'chitskii; Zhur。 Eksp. i Teoret. Fiz 35, 322 (1958); Soviet Phys。 JETP $8,224(1959)$ 
$58 \mathrm{Ba} 7$ A. I. Baz, N. M. Kul1kova, L. E. Lazareva, N. V. Nikitina, V. A. Semenov; Int. Conf. Peaceful Uses of Atomic Energy 15, 2037 (1958)

$58 \mathrm{Be} 1$ Wo Lo Bendel, J. McElhinney, R. A. Tobin; Phys. Rev • 111,1297 ( 1958 )

$58 \mathrm{Be} 2$ W. Bertozzi, F. R. Paolini, C. P. Sargent; Phys. Rev. $110,790(1958)$

$58 \mathrm{Be} 3$ o. Beckman, R。 Sandstrom; Nuclear Phys. 5, 595 (1958)

58 Bo 1 O. V. Bogdankevich, B. S. Dolbilkin, L. E. Lazareva,

F. A. Nikolaev; PICNP 697 (1958)

$58 \mathrm{Br} 1$ P. Brix, U. Hegel, K. H. Lindenberger, D. Quitmann;

Z. Physik 150, 461 (1958)

$58 \mathrm{Bu} 1$ N. A. Burgov, Yu. V. Terekhov; J. Nucl. Energy 7, $247(1958)$

$58 \mathrm{Ca} 1$ J. Ho Carver, W. Turchinetz; Proc. Phys. Soc. 71, $613(1958)$

$58 \mathrm{Ca} 2$ S. Cavallaro, V. Emma, C. Milone, A. Rubbino; Nuovo Cimento 9,736 (1958)

$58 \mathrm{Ch} 1$ I. V. Chuvilo, V. G. Shevchenko; Zhur. Eksp. i Teoret. Fiz. 34,593 (1958); Soviet Phys. JETP 7, 410 (1958)

$58 \mathrm{Ch} 2$ B. G. Chidley, L. Katz, S. Kowalski; Can. J. Phys. 36, 407 ( 1958$)$

58 Co 1 G. Cortini, C. Milone, A. Rubbino, Nuovo Cimento 9, 85 (1958)

58 Co 2 G. Cortini, C. Milone, R. Rinzivil1o, C. Tribuno; Nuovo Cimento 9, 188 (1958)

$58 \mathrm{Du} 1$ R. B. Duffield, R. A. Schmitt, R. A. Sharp; Int. Conf。 Peaceful Uses of Atomic Energy 15, 678 (1958)

$58 \mathrm{Em} 1$ V. Emma, C. Milone, A. Rubbino; Nuovo Cimento 9, 343 (1958)

$58 \mathrm{Fa} 1$ U. Farinel11, F. Ferrero, R。 Malvano, S. Menardi, E. Silva; Phys. Rev. 112, 1994 (1958)

$58 \mathrm{Fa} 2 \mathrm{H}$. Faissner, F. Gonnenwein; Z. Physik 153, 257 (1958)

$58 \mathrm{Fe} 1 \mathrm{~F}$. Ferrero, R。 Malvano, S. Menard1, O. Terracini; Nuclear Phys. 9, 32 (1958)

$58 \mathrm{Fu} 1$ E. G. Fuller, B. Petree, M. S. Weiss; Phys. Rev. 112 , $554(1958)$

$58 \mathrm{Fu} 2$ E. G. Fuller, M. S. Weiss; Phys. Rev. 112, 560 (1958)

$58 \mathrm{Fu} 3$ E. G. Fuller, E. Hayward; Phys. Rev. Letters 1, 465 (1958)

58 Go 1 A. N. Gorbunov, V. M. Sniridonov; Zhur. Eksp. i Teoret. Fiz 34, 862 (1958); Soviet Phys.JETP 7, 596 (1958)

58 Go 2 A. N. Gorbunov, V. M. Spiridonov; Zhur. Eksp. 1 Teoret. Fiz 34, 866 (1958); Soviet Phys. JETP 7, 600 (1958)

58 Go 3 J. Goldemberg, L. Marquez; Nuclear Phys. 7, 202 (1958)

$58 \mathrm{Gr} 1$ G. M. Griffiths; Proc. Phys. Soc. (London) 72, 337 (1958)

$58 \mathrm{Gu} 1 \mathrm{~F}$. Gudden, J. Eichler; Z. Physik 150, 436 (1958)

$58 \mathrm{Ha} 1$ A. Halsteinslid, K. Nybo, R. B. Mollerud; Univ. Bergen Arb. Naturv. R. 15, 1 (1958)

58 Ho 1 A. Hofmann, P. Stoll; Helva. Phys. Acta 31, 591(1958)

$58 \mathrm{Ka} 1$ L. Katz, G. B. Chidley; Nuclear Reactions at Low and Medium Energies (Dub. Academy of Science, USSR) 371 (1958)

$58 \mathrm{Ke} 1$ L. Keszthely1, J. Ero, Nuclear Phys. 8, 650 (1958)

$58 \mathrm{Ki} 1$ H. J. King, L. Katz; Can. J. Phys. 36, 415 (1958)

$58 \mathrm{Li} 1$ D. L. Livesey, I. G. Main, Nuovo Cimento 10,590 (1958) 
$58 \mathrm{Ma} 1$ V. N. Malkov; Zhur. Eksp. i Teoret. Fiz. 34, 1406 (1958); Soviet Phys. JETP 7, 973 (1958)

58 Me 1 F. R. Metzger, C. P. Swann, V. K. Rasmussen; Phys. Rev. $110,906(1958)$

$58 \mathrm{Mi} 1$ C. Milone, S. Milone-Tamburino, R. Rinzivilio, A. Rubbino, C. Tribuno; Nuovo Cimento 7, 729 (1958)

58 Mo 1 J. Moffatt, M. W. Stringfellow; Phil. Mag。3, 540 (1958)

58 Mo 2 J. Moffatt, J. J. Thresher, G. C. Weeks, R. Wilson; Proc. Roy. Soc. (London) A244, 245 (1958)

58 On 1 T. R. Ophe1, I. F. Wright; Proc. Phys. Soc. 71,389 (1958)

58 On 2 T. R. Ophel, Proc. Phys. Soc. 72,321 (1958)

58 Ox 1 C. L. Oxley, Phys. Rev. 110, 733 (1958)

$58 \mathrm{Ra} 1 \mathrm{~V}$. K. Rasmussen, F. R. Metzger, Phys. Rev. 110, 154 (1958)

$58 \mathrm{Rh} 1$ J。 L. Rhodes, W. E. Stephens; Phys.Rev. 110, 1415 (1958)

58 Ry 1 T. W. Rybka, L. Katz; Phys. Rev. 110, 1123 (1958)

58 Si I E. Silva, J. Goldemberg; Phys. Rev• 110, 1102 ( 1958 )

58 Si 2 E. Silva, J. Goldemberg, P. S. Smith; Nuovo Cimento 9, 17 (1958)

$58 \mathrm{Si} 3$ A. K. M. Siddiq, R. N. H. Haslam; Can. J. Phys. 36, $963(1958)$

58 Si 4 E. Silva, J. Goldemberg; An. Acad. Brasil. Cienc. 30 , $51(1958)$

$58 \mathrm{Sn} 1$ B. M. Spicer, H. H. Thies, J. E. Baglin, F. R. Allum; Australian J. Phys. 11, 298 (1958)

58 Sn 2 B. M. Spicer, F. R. Allum, J. E. Baglin, H. H. Thies; Australian J. Phys. 11, 273 (1958)

58 T 1 G. W。 T\&utfest, Phys. Rev. 110,708 (1958)

$58 \mathrm{Ta} 2$ C. A. Tatro, T. R. Palfrey, Jr., R. M. Whaley, R. O. Haxby; Phys. Rev. 112, 932 (1958)

58 To I R. Tobin, J. McElhinney, L. Cohen; Phys. Rev. 110, 1388 (1958)

58 To 2 M. E. Toms, J. McElhinney; Phys. Rev. 111, 561 (1958)

58 Wh 1 A. Whetstone, J. Halnern; Phys. Rev. 109, 2072 (1958)

58 Wh 2 C. Whitehead, W. R. McMurray, M. J. Aitken, N. Middlemas, C. H. Collie; Phys. Rev. 110,941 (1958)

58 Wo 1 M. M. Wolff, W. E. Stephens; Phys. Rev. 112, 890 ( 1958 )

58 Ya 1 T. P. Yavor, Zhur. Ekso. i Teoret. Fiz. 34, 1420 (1958); Soviet Phys. JETP 7, 983 (1958)

58 Zi 1 E. Ziegler, Z. Physik 152, 566 (1958)

$59 \mathrm{Ag} 1 \mathrm{~A}$. Agodi, E. Eberle, L. Sertorio; Nuovo Cimento 13, $1279(1959)$

$59 \mathrm{Au} 1$ L. B. Aul1, P. C. Reinhardt, W. D. Whitehead; Nuclear Phys. 13, 292 (1959)

$59 \mathrm{Ba} 1$ W. C. Barber, F. E. Gudden; Phys. Rev. Letters 3, $219(1959)$

$59 \mathrm{Ba} 2$ E. B. Bazhanov, Zhur. Eksp. i Teoret. Fiz. 37, 374 (1959); Soviet Phys. JETP 10, 267 (1960)

$59 \mathrm{Ba} 3$ W. C. Barber, W. D. George; Phys. Rev.116, 1551 (1959) 
$59 \mathrm{Ba} 4$ A. B。 Baerg, R. M. Bartholomew, F. Brown, L。 Katz, S. B。 Kowalski; Can。J。 Phys。 37, 1418 (1959); P。 M. Dyson, J。M。 Kennedy, E. Vogt; Can。J。 Phys。37, 1435 (1953)

59 Bo 1 M. Bormann, Ho Neuert; Z。 Naturforsch。14a, 922 (1959)

$59 \mathrm{Br} 1$ Po Brix, A。 Kording, K. H. Lindenberger; Z. Physik $154,569(1959)$

$59 \mathrm{Br} 2$ P。 Brix, E。 Ko Maschke; Z。 Physik 155, 109 (1959)

$59 \mathrm{Bu} 1 \mathrm{~N}$. A. Burgov, G。 V。 Danilyan, B。 S。 Dolbilkin, L。 E。 Lazareva, F.A. Nikolaev; Zhur。Eksp. i Teoret。Fiz. 37, 1811 (1959); Soviet Phys。 JETP 10, 1278 (1960)

$59 \mathrm{Ca} 1$ Jo Ho Carver, Go A. Jones; Nuclear Phys. 11, 400 (1959)

$59 \mathrm{Ca} 2$ J. Ho Carver, W。 Turchinetz; Proc。 Phys. Soc。 73, 110 (1959)

59 Ca 3 J. H. Carver, Wo Turchinetz; Proc。Phys. Soc。 73, 69 (1959)

$59 \mathrm{Ca} 4$ J。 H. Carver, Wo Turchinetz; Proc。 Phys。Soc。 73, 585 (1959)

$59 \mathrm{Ch} 1$ V。 Po Chizhov, L. A。 Kul'chitskii; Zhur。 Eksp. i Teoret。 Fiz。 36, 345 (1959); Soviet Phys。 JETP 9, 239 (1959)

59 Co 1 Lo D。 Cohen, W. E。 Stephens; Phys. Rev. Letters 2, 263 (1959)

59 Co 2 S。 G。 Cohen, P. S。 Fischer, E。 K. Warburton; Phys. Rev. Letters 3,433 (1959)

59 Co 3 Go Cortini, C。 Milone, T. Pana, R。 Rinzivillo; Nuovo Cimento 14, 54 (1959)

59 Co 4 L. Cohen, R。A. Tobin; Nuclear Phys。 14, 243 (1959)

59 Co 5 L. Cohen, R。A。 Tobin, J. McElhinney; Phys。 Rev. $114,590(1959)$

59 Co 6 S。 Go Cohen; Nuovo Cimento 14, 931 (1959)

59 Da 1 F。 Daublin, F。 Berthold, P. Jensen; Z. Naturforsch. 14a, 208-211(1959)

59 Di 1 Go DiCaporiacco, Mo Mando; Nuovo Cimento 13, 522 (1959)

$59 \mathrm{Du} 1$ Jo Dular, G。 Kernel, M. Kregar, M。V。 Mihailovic, G. Pregl, M. Rosina, C. Zupancic; Nuclear Phys。 14, $131(1959)$

59 Dy 1 P. Dyal, J。 P. Humme 1; Phys。 Rev。115, 1264 (1959)

59 Em 1 V. Emma, C. Milone, R。 Rinzivillo; Nuovo Cimento 14, 1149 (1959)

59 Em 2 V. Emma, C. Milone, R. Rinzivillo, A. Rubbino; Nuovo Cimento 14,62 (1959)

$59 \mathrm{Fa} 1$ U。 Farinelli, Fo Ferrero, S. Ferroni, R. Malvano, E. Silva; Nuovo Cimento 12,89 (1959)

$59 \mathrm{Fe} 1 \mathrm{~F}$. Ferrero, So Ferroni, R. Malvano, S. Menardi, E. Silva; Nuovo Cimento 11, 410 (1959)

$59 \mathrm{Fe} 2 \mathrm{~F}$. Ferrero, R. Malvano, E。 Silva, J。 Goldemberg, G. Moscati; Nuclear Phys. 10, 423 (1959)

59 Fr 1 J。 I。 Friedman; Phys。Rev。116, 1257 (1959)

$59 \mathrm{Ge} 1$ D. S. Gemmel1, A. H. Morton, E. W. Titterton; Nuclear Phys. 10, 33 (1959)

$59 \mathrm{Gi} 1 \mathrm{~J} . \mathrm{H} \cdot \mathrm{Gibbons,} \mathrm{R.} \mathrm{L。} \mathrm{Macklin,} \mathrm{J.} \mathrm{B.} \mathrm{Marion,} \mathrm{H。} \mathrm{W.}$ Schmitt; Phys。Rev。114, 1319 (1959)

59 Go 1 H。 E. Gove, A. E。 Litherland, R. Batchelor; Phys. Rev. Letters 3,177 (1959) 
$59 \mathrm{Ha} 1 \mathrm{H} \cdot \mathrm{J} \cdot \mathrm{Hay}, \mathrm{J} \cdot \mathrm{B} \cdot$ Warren; Can. J。 Phys. 37, 1153 (1959)

$59 \mathrm{Ha} 2$ F. I. Havlicek; Nuovo Cimento 13, 969 (1959)

59 Ik 2 N. Ikeda, K. Yoshihara; Radioisotopes (Tokyo) 8, 24 (1959)

$59 \mathrm{Ki} 1$ H. King, L。 Katz; Can. J. Phys. 37, 1357 (1959)

59 Ko 1 Jo Kockum, N. Starfelt; Nuclear Instruments 5, 37 ( 1959)

59 Ko 2 A. Po Komar, T. N. Dragnev; Dokl. Akad. Nauk SSSR 126, 1234 (1959); Soviet Phys。 Doklady 4, 653 (1959)

$59 \mathrm{Ku} 1$ L. A. Kul'chitski, V. Presperin; Zhur。Eksp. i Teoret。 Fiz. 371524 (1959); Soviet Phys. JETP 10, 1082 (1960)

$59 \mathrm{Ku} 2 \mathrm{Chi-Ti}$ Kuo, B. S. Ratner; Dokl. Akad. Nauk SSSR 125, 761 (1959); Soviet Phys. Doklady 4, 369 (1959)

59 La 1 M. Langevin, A. Bussiere de Nercy; J. Phys. Radium 20, 831 (1959)

59 Lo 1 K. H. Lokan; Proc. Phys. Soc. 73, 697 (1959)

$59 \mathrm{Ma} 1$ E. D。 Makhnovskii; Zhur. Eksp. i Teoret. Fiz. 36, 739 (1959); Soviet Phys. JETP 9, 519 (1959)

59 Me 1 F. R. Metzger, W. D. Todd; Nuclear Phys. 13, 177 (1959)

59 Mi 1 M. V. Mihailovic, G. Pregl, G. Kernel, M. Kregar; Phys. Rev. 114, 1621 (1959)

$59 \mathrm{Mi} 2$ C. Milone; Phys. Rev. Letters 3, 43 (1959)

$59 \mathrm{Mi} 3$ C. Milone, A. Rubbino. Nuovo Cimento 13, 1035 (1959)

$59 \mathrm{Mu} 1$ N. ilutsuro, i. Ohnuki, K. Sato, K. Kageyama, M. Kimura; J. Phys. Soc. Japan 14, 1457 (1959)

$59 \mathrm{Mu} 2 \mathrm{~N}$. Mutsuro, Y. Ohnuki, K. Sato, M. Kimura; J. Phys. Soc. Japan 14, 1649 (1959)

$59 \mathrm{Na} 1$ T. Nakamura, K. Takamatsu, K. Fukunaga, M. Yata, S. Yasumi; J. Phys. Soc. Japan 14, 693 (1959)

59 Oc 1 J. OiConnel1, P. Dyal, J. Goldemberg; Phys. Rev. 116, $173(1959)$

$59 \mathrm{~Pa} 1 \mathrm{R}$. W. Parsons, L. Katz; Can. J. Phys. 37, 809 (1959)

$59 \mathrm{~Pa} 2 \mathrm{R}$. W. Parsons; Can。 J. Phys. 37, 1344 (1959)

$59 \mathrm{~Pa} 3$ P. Paul, U. Stierlin; Nuclear Phys. 13, 576 (1959)

$59 \mathrm{Pe} 1 \mathrm{~A} . \mathrm{S}$. Penfold, E. L. Garwin; Phys. Rev. 114, 1139 (1959)

59 Pe 2 A. S. Penfold, E. L. Garwin; Phys. Rev • 114, 1324 (1959)

$59 \mathrm{Pe} 3$ A. S. Penfold, E. L。 Garwin; Phys. Rev. 115, 420 (1959)

$59 \mathrm{Pe} 4$ S. Penner, J。 E。 Leiss; Phys. Rev • 114, 1101 (1959)

$59 \mathrm{Pe} 5$ A. S. Penfold, E. L. Garwin; Phys。 Rev. 116, 120 (1959)

$59 \mathrm{Ra} 1$ V. K. Rasmussen, F. R. Metzger, C. P. Swann; Nuclear Phys. 13, 95 (1959)

59 Ro 1 T. A. Romanowski, V. W. Voelker; Phys. Rev. 113, 886 (1959)

59 Ro 2 J. P. Roalsvig, R. N.H. Haslam, D. J. McKenzie; Can. J. Phys. 37, 607 (1959)

59 Ro 3 Jo Po Roalsvig, R. N. H. Haslam, L. D. Skarsgard,

E. E. Wuschke; Can. J. Phys. 37, 722 (1959)

$59 \mathrm{Sa} 1 \mathrm{D}$. Sadeh; Compt. Rend. 249 , 531 (1959)

59 Se 1 R. E. Segel; Phys. Rev. 113, 844 (1959)

59 Se 2 W. Sebaoun, H. Gauvin; Compt. Rend. 248, 791 (1959)

59 Sw 1 C. P. Swann, V. K. Rasmussen, F. R. Metzger; Phys. Rev. 114, 862 (1959)

$59 \mathrm{Ta} 1 \mathrm{~N}$. W. Tanner, G. C. Thomas, W. E. Meyerhof; Nuovo Cimento $14,257(1959)$ 
59 Th 1 H. H. Thies, B. M. Spicer, J.E.E. Baglin; Australian J. Phys. 12, 21 (1959)

$59 \mathrm{Va} 1$ I. Sh. Vashakidze, T. I. Konaleishvili, G. A. Chilashvili; Zhur. Eksp. i Teoret. Fiz 37, 750 (1959); Soviet Phys. JETP $10,535(1960)$

$60 \mathrm{Ai} 1$ M. J. Aitken, N. Middlemas; Phys. Rev. 117, 1111 (1960)

60 Al 1 Ya. Alksnis, U. Ulmanis; Latv. PSR Zinat. Akad. Vestis 3 $(152), 71(1960)$

60 As 1 R. F. Askew, A. P. Batson; Nuclear Phys. 20, 408 (1960)

$60 \mathrm{Ba} 1$ E. B. Bazhanov; Zhur. Eksp. i Teoret. Fiz. 38, 267 (1960); Soviet Phys. JETP 11, 193 (1960)

$60 \mathrm{Ba} 2$ W. C. Barber, T. Wiedling; Nuclear Phys. 18, 575 (1960)

$60 \mathrm{Ba} 3$ E. B. Bazhanov, L. A. Kul'chitskii; Zhur. Eksp. i Teoret. Fiz. 38, 1685 (1960); Soviet Phys. JETP 11, 1215 (1960)

$60 \mathrm{Ba} 4$ W. C. Barber, F. Berthold, G. Fricke, F. E. Gudden; Phys. Rev. 120, 2081 (1960)

$60 \mathrm{Ba} 5$ W. C. Barber, V. J。 Vanhuyse. Nuclear Phys. 16, 381 (1960)

$60 \mathrm{Ba} 6$ W. C. Barber, V. J. Vanhuyse. J. Phys。 Radium 21, 299 (1960)

$60 \mathrm{Ba} 7$ D. Balfour, D. C. Menzies; Proc. Phys. Soc. 75, 543(1960)

$60 \mathrm{Ba} 8 \mathrm{M}$. A. Bak, K. A. Petrzhak, Tei-Mei Chen; Izv. Akad. Nauk SSSR 24, $818(1960)$

$60 \mathrm{Be} 1$ W. Bertozzi, P. T. Demos, S. Kowalski, F. Paolini, C. P. Sargent; PICNS 746, (1960)

60 Bo 1 B. A. Bochagov, A. P. Komar, G. E. Solaykin; Zhur. Eksp. i Teoret. Fiz. 38, 1374 (1960); Soviet Phys. JETP $11,990(1960)$

60 Bo 2 O. V. Bogdankevich, L. E. Lazareva, A. M. Maiseev; Zhur. Eksp. i Teoret. Fiz. 39, 1224 (1960); Soviet Phys. JETP $12,853(1961)$

60 Bo 3 E. C. Booth; Nuclear Phys. 19, 426 (1960)

$60 \mathrm{Br} 1$ C. Broude, H. E. Gove; PICNS 754 (1960)

$60 \mathrm{Bu} 2$ A. Bussiere de Nercy; Compt. Rend. 250, 1252 (1960)

$60 \mathrm{Bu} 3$ A. Bussiere de Nercy, M. Langevin; J. Phys. Radium 21, $293(1960)$

$60 \mathrm{Ca} 1$ E. E. Carroll, Jr., W. E. Stephens; Phys. Rev. 118, $1256(1960)$

$60 \mathrm{Ca} 2$ J. H. Carver, R. B. Taylor, W. Turchinetz; Aust. J. Phys. $13,617(1960)$

$60 \mathrm{Ch} 1$ V. P. Chizhov; Zhur. Eksp. i Teoret. Fiz. 38, 809 (1960); Soviet Phys. JETP 11, 587 (1960)

$60 \mathrm{Ch} 2$ R. E. Chrien, A. H. Benade; Phys. Rev. 119, 748 (1960)

$60 \mathrm{De} 1$ B. I. Deutch, F. R. Metzger, F. J. Wilhelm; Nuclear Phys. 16, 81 (1960)

$60 \mathrm{De} 2$ N. N. Delyagin; Zhur. Eksp. i Teoret. Fiz. 38, $1111(1960)$; Soviet Phys. JETP 11, 803 (1960)

60 Do 1 H. G. Dosch, K. H. Lindenberger, P. Brix; Nuclear Phys. 18, 615 (1960)

60 Ed 1 R. D. Edge; Phys. Rev. 119, 1643 (1960) 
60 Em 1 V. Emma, C. Milone, A. Rubbino, A. Malvano; Nuovo Cimento 17,365 (1960)

$60 \mathrm{Em} 2$ V. Emma, C. Milone, A. Rubbino, Phys. Rev. 118, 1297( 1960)

$60 \mathrm{Fa} 1$ R. W. Fast, D. A. Flournoy, R. S. Tickle, W. D. Whitehead; Phys. Rev. 118, 535 (1960)

60 Fe 1 F. Ferrero, S. Ferroni, R. Malvano, S. Menardi, E。 Silva; Nuclear Phys。 15, 436 (1960)

$60 \mathrm{Fe} 2$ B. T. Feld, B. Co Maglic, J. Parks; Nuovo Cimento 17, Suppl。2, 241 (1960)

60 F1 1 P. A. Flournoy, R. S. Tickle, W. D. Whitehead; Phys. Rev. 120, 1424 (1960)

60 Fo 1 B. Forkman, S.A.E. Johansson; Nuclear Phys. 20, 136 (1960)

60 Fo 2 B. Forkman, I. Wahlstrom; Arkiv Fysik 18, 339 (1960)

$60 \mathrm{Ga} 1$ J。A. Galey; Phys. Rev。117, 763 (1960)

60 Ga 2 M. Garnier, H. Gauvin, W. Sebaoun; J. Phys. Radium 21, $893(1960)$

$60 \mathrm{Ge} 1$ K. N. Geller; Phys. Rev. 120, 2147 (1960)

$60 \mathrm{Ge} 2 \mathrm{~K} \cdot \mathrm{N} \cdot \mathrm{Geller}$, J. Halpern, E. G. Muirhead; Phys. Rev。 $119,716(1960)$

60 Ge 3 K. N. Keller, J. Haloern, E. G. Muirhead; Dhys. Rev . 118, $1302(1960)$

60 Go 1 V. I. Gol'danskii, O. A. Karpukhin, A. V. Kutsenke, V. V. Pavlovskaya; Zhur. Eksp. i Teoret. Fiz. 38, 1695 (1960); Soviet Phys。 JETP 11, 1223 (1960)

60 Go 2 A. N. Gorbunov, F. D. Denisov, V. A. Kolutkhin; Zhur. Eksp. i Teoret. Fiz. 38, 1084 (1960); Soviet Phys. JETP $11,783(1960)$

60 Gr 1 V. M. Grizhko, D. I. Sikora, V. A. Shkoda-U1'yanov, A. D. Abramenkov, B. I. Shramenko, A. N. Fisun; Zhur. Eksp. i Teoret. Fiz. 38, $1370(1960)$; Soviet Phys. JETP 11, $987(1960)$

$60 \mathrm{Ha} 1$ F. I. Havlicek; Nuovo Cimento 18, 65 (1960)

$60 \mathrm{He} 1$ D. F. Hebbard; Nuclear Dhys. 15, 289 (1960)

$60 \mathrm{He} 2$ D. F. Hebbard, J. L. Vogl; Nuclear Phys. 21, 652 (1960)

60 Ho 1 P. Horvat, J. Dahor, M. Vakse1j; Nuclear Phys。 16, 90 (1960)

60 Is 1 D. Isabelle, G. Bishop; Compt。Rend. 251, 697 (1960)

$60 \mathrm{Ja} 1$ D. Jamnik, D. Axel; Phys. Rev. 117, 194 (1960)

$60 \mathrm{Ka} 1$ D. K. Kainov, Yu. K. Shubnyi; Zhur. Eksp. i Teoret. Fiz. 39, 888 (1960); Soviet Phys。 JETP 12, 615 (1961)

$60 \mathrm{Ki} 1$ Jo D. King, Wo Jo McDonald; Nuclear Phys. 19, 94 (1960)

$60 \mathrm{Ki} 2$ J. D. King, R. N. H. Haslam, R. W. Parsons. Can. J。 Phys. $38,231(1960)$

$60 \mathrm{Ki} 3$ M. Kimura, K. Shoda, N. Mutsuro, T. Tohei, K. Sato, K. Kuroda, K. Kuriyama, T. Ariba; J. Phys. Soc. Japan 15 , $1128(1960)$

$60 \mathrm{Ki} 4$ J. D. King, R. N. H. Haslam, W. J० McDonald; Can。 J。 Phys. $38,1069(1960)$

60 Ko 1 A. P. Komar, Ya. Krzhemenek, I. P. Yavor; Dokl. Akad. Nauk SSSR 131, 283 (1960); Soviet Phys. Doklady 5, 295(1961)

60 Ko 2 A. D. Komar, Ya. Krzhemenek, I. D. Yavor; Dok1. Akad. Nauk SSSR 135, 291 (1960); Soviet Phys。 Doklady 5, 1234 (1961) 
60 Ko 3 A. D. Komar, E. D. Makhnovskil; Dokl. Akad. Nauk SSSR 135, 52 (1960); Soviet Phys. Doklady 5, $1229(1961)$

60 Ko 4 A. P. Komar, E. D. Makhnovskii, V. P. Puddubnov; Dok1. Akad. Nauk SSSR 133, 797 (1960); Soviet Phys. Doklady 5, $824(1961)$

60 Ko 5 A. P. Komar; PICNS 494 (1960)

60 Ko 6 A. P. Komar; PICNS 875 (1960)

$60 \mathrm{Kr} 1$ R. W. Krone, J. J. Singh; Phys. Rev. 117, 1562 (1960)

$60 \mathrm{Ku} 1$ Kuo Ch'i-di, B. S. Ratner; Zhur. Eksp. i Teoret. Fiz. 39, 1578 (1960); Soviet Phys JETP 12, 1098 (1961)

$60 \mathrm{Ku} 2$ L. A. Kul'chitskii, V. Presperin; Zhur. Eksp. i Teoret. Fiz. 39, 1001 (1960); Soviet Phys. JETP 12, 696 (1961)

60 Li 1 N. V. Linkova, R. M. Osokina, B. S. Ratner, R. Sh. Amirov, V. V. Akindinov; Zhur. Eksp. I Teoret. Fiz. 38, 780 (1960); Soviet Dhys. JETP 11, 566 (1960)

60 Li 2 K. H. Lindenberger, J. A. Scheer; Z. Physik 158, 111 ( 1960$)$

$60 \mathrm{Ma} 1 \mathrm{E}$. D. Makhnovskii; Zhur. Eksp. i Teoret. Fiz. 38, 96 (1960); Soviet Phys. JETP 11, 70 (1960)

$60 \mathrm{Ma} 2$ B. Mainsbridge; Nuclear Phys. 21, 1 ( 1960 )

$60 \mathrm{Me} 1 \mathrm{~F}$. R. Metzger, C. P. Swann, V. K. Rasmussen; Nuclear Phys. $16,568(1960)$

$60 \mathrm{Me} 2$ F。 R。 Metzger; J. Franklin Institute 270, 138 (1960)

$60 \mathrm{Mi} 1$ C. Milone; Dhys. Rev. 120, 1302 (1960)

$60 \mathrm{Mi} 2$ M. Miwa, M. Yamanouchi; J. Phys. Soc. Japan 15, $947(1960)$

60 Mo 1 W. L. Mouton, P. B. Smith; Nuclear Phys. 16, 206 (1960)

$60 \mathrm{Mu} 1 \mathrm{~N}$. Mutsuro, K. Sato, M. Mishina; J. Phys。 Soc. Japan $15,358(1960)$

60 Ne 1 J. O. Newton; Nuclear Phys. 21, 529 (1960)

$60 \mathrm{Pr} 1$ D. G. Proctor, W. H. Voelker; Phys。Rev. 118, 217 ( 1960$)$

$60 \mathrm{Dr} 2$ L. I. Prokhorova, G. N. Smirenkin; Atom. Energiya 8, $457(1960)$

$60 \mathrm{Re} 1$ K. Reibe1, A. K. Mann; Phys. Rev. 118, 701 (1960)

$60 \mathrm{Re} 2$ J. M. Reid, B. Lalovic; Proc. Phys. Soc. (London) 76 , $65(1960)$

60 Ro 1 H. Jo Rose; Nuclear Phys. 19, 113 (1960)

60 Ro 2 H. J. Rose, F. Riess, W. Trost; Nuclear Phys. 21, 367

60 Ro 3 M. Rozkos, M. Smrcka, O. Jakubcek; Czech. J. Phys. $10,129(1960)$

60 Ro 4 J. P. Roalsvig, R. N. H. Haslam, J. L. Bergsteinsson; Can. J. Phys. 38, $320(1960)$

$60 \mathrm{Sa} 1$ D. Sadeh; Phys. Rev. Letters 4, 75 (1960)

$60 \mathrm{Sa} 2$ D. Sadeh; Compt. Rend. 250, 1632 (1960)

60 St 1 p. C. Stein, A. C. CAian, A. Wattenberg, R. Weinstein; Phys. Rev. 119, 348 (1960)

$60 \mathrm{Ta} 1 \mathrm{R}$. B. Taylor; Nuclear Phys. 19, 453 (1960)

$60 \mathrm{Ta} 2$ G. Tamas, J. Miller, C. G. Schuhl, C. Tzara; J. Phys. Radium 21, $532(1960)$

60 Ta 3 E. Takekoshi, J. Phys. Soc. Japan 15, 2129 (1960) 
$60 \mathrm{Th} 1$ H. H. Thies, B. M. Spicer; Australian J. Phys. 13, $505(1960)$

60 To 1 R. A. Tobin; Phys. Rev。120, 175 (1960)

$60 \mathrm{Va} 1$ V. J. Vanhuyse, G. J. Vanpraet; J. Phys. Radium 21, $290(1960)$

$60 \mathrm{Wa} 1$ I. Wahlstrom, B. Forkman; Arkiv Fysik 18, 83 (1960)

60 Wa 2 T. G. Walker, W. T. Morton; Proc. Phys. Soc. 75, $4(1960)$

60 Wy 1 J. M. Wyckoff, H. W. Koch; Phys. Rev. 117, 1261 (1960)

$60 \mathrm{Ya} 1$ S. Yasumi, M. Yata, K. Takamatsu, A. Masaike, Y。 Masuda;

J. Phys. Soc. Janan 15, 1913 (1960)

$60 \mathrm{Zi} 1$ B. Ziegler; Nuclear Phys. 17, 238 (1960)

61 Ak 1 A. F. Akkerman, D. K. Kaipov, Yu. K. Shubnyi; Zhur • Eksp. i Teoret. Fiz. 40, 1031 (1961); Soviet Phys. JETP $13,725(1961)$

$61 \mathrm{Ba} 1$ J. E. E. Baglin, M. N. Thompson, B. M. Spicer; Nuclear Phys. 22, 207 (1961)

$61 \mathrm{Ba} 2$ R. G. Baker, K. G. McNeill; Can. J. Phys. 39, 1158 (1961)

$61 \mathrm{Ba} 3$ P. S. Baranov, L. I. Slovokhotov, G. A. Sokol, L. N. Shtarkov; Zhur. Eksp. i Teoret. Fiz. 41, 1713 (1961); Soviet Phys. JETP 14, 1219 (1962)

61 Be 1 A. K. Berzin, R. P. Meshcheryakov; Zhur. Eksp. i Teoret. Fiz. 41, 1013 (1961); Soviet Phys. JETP 14, 721 (1962)

$61 \mathrm{Be} 2 \mathrm{R}$. E. Benenson, L. J. Lidofsky; Phys. Rev• 123, 939 (1961)

$61 \mathrm{Bi} 1 \mathrm{G}$. R. Bishop, G. A. Proca; J. Phys. Radium 22, 541 (1961)

61 Bo 1 O.Borello, S.Costa, F.Ferrero; Nuclear Phys. 27, 25 (1961)

61 Bo 2 B. A. Bochagov, A. P. Komar, G. E. Solyakin, V. I. Fadeev; Atom. Energiya 11, 540 (1961)

61 Bo 3 O. A. Borello; Rev• Un. Mat. Argent. As. Fis. Argent. 19, $251(1961)$

61 Bo 4 P. Bounin, G. R. Bishop; J. Phys. Radium 22, 555 (1961)

$61 \mathrm{Br} 1$ P. Brix, H. Fuchs, K. H. Lindenberger, C. Salander;

Z. Physik 165,485 (1961)

$61 \mathrm{Bu} 1$ J. W. Butler; Phys。 Rev•123, 873 (1961)

$61 \mathrm{Bu} 2 \mathrm{G} \cdot \mathrm{R}$. Burleson; Phys. Rev. 121, 624 (1961)

$61 \mathrm{Ca} 1$ J. H. Carver, G. A. Jones; Nuclear Phys。 24, 607 (1961)

$61 \mathrm{Ca} 2$ J. H. Carver; Proc. Phys. Soc. 77, 417 (1961)

$61 \mathrm{Ce} 1 \mathrm{R}$. J. Cence, B. J. Moyer; Phys. Rev. 122, 1634 (1961) (1961)

61 Co 1 S. G. Cohen, P. S. Fisher, E. K. Warburton; Phys. Rev. 121,858 (1961)

61 Co 2 G. E. Coote, W.E.Turchinetz, I.F.Wright; Nuclear Phys. $23,468(1961)$

$61 \mathrm{Cr} 1$ H. Crannell, R. Helm, H. Kendall, J. Oeser, M. Yearian; Phys. Rev. 123, 923 (1961)

61 De 1 H. G. DeCarvalho, A. G. DaSilva, J. Goldemberg;

Nuovo Cimento 19,1131 (1961)

61 De 2 H. G. DeCarvalho, A. Celano, G. Cortini, R. Rinzivillo, G. Ghigo; Nuovo Cimento 19,187 (1961)

61 De 3 B. I. Deutch, F. R. Metzger; Phys. Rev. 122, 848 (1961)

61 De 4 A. B. DeNercy; J. Phys. Radium 22, 535 (1961) 
61 Du 1

61 Ec 1

$61 \operatorname{Em} 1$

61 Fi 1

61 Fo 1

61 Go 1

$61 \mathrm{Ha} 1$

$61 \mathrm{He} 1$

$61 \mathrm{He} 2$

61 Ho 1

$61 \mathrm{Hu} 1$

61 Is 1

$61 \mathrm{Ja} 1$

$61 \mathrm{Ja} 2$

61 Jo 1

$61 \mathrm{Ke} 1$

$61 \mathrm{Ke} 2$

$61 \mathrm{Ke} 3$

$61 \mathrm{Ki} 1$

61 Ko 1

$61 \mathrm{Ku}$

61 La 1

$61 \mathrm{Ma} 1$

$61 \mathrm{Ma} 2$

$61 \mathrm{Me} 1$

$61 \mathrm{Mi} 1$

61 Mo 1

61 My 1

$61 \mathrm{Na} 1$

61 No 1

$61 \mathrm{Pe} 1$

61 Po 1 R. A. Pope, D 24, $657(1961)$

$61 \operatorname{Pr} 1$ V. Presperin, L. A. Kul'chitskii; Zhur. Eksp. i Teoret. Fiz. 41, 60 (1961); Soviet Phys. JETP 14, 46 (1962)

61 Ra 1 V. K. Rasmussen, F. R. Metzger, C. P. Swann; Phys. Rev. 123, 1386 (1961)

A. C. Eckert, E. F. Shrader; Dhys. Rev. 124, 1541

(1961)

Nuovo Cimento $22,135(1961)$

E. Finch, U. Hegel; Z. Dhysik 162, 154 (1961)

B. Forkman; Nuclear Dhys. 23, 269 (1961)

H. E. Gove, A. E. Litherland, R. Batchelor; Nuclear nhys. 26, 480 (1961)

W. D. Hamilton, B. S. Sood; Nuclear Phys. 27, 66 (1961)

R. E. Hester, W. A. S. Lamb; Phys. Rev. 121, 584 (1961)

U. Hegel, E. Finckh; Z. Physik 162, 142 (1961)

C. P. Ho, E. L. Iloff; Nuclear Phys. 27, 234 (1961)

J. P. Humme 1, Dhys. Rev. 123,950 (1961)

D. S. Isabelle, G. R. Bishop; J. Phys. Radium 22, $548(1961)$

M. J. Jakobson; Phys. Rev. 123, 229 (1961)

A. N. James; Nuclear Phys. 24, 675 (1961)

W. John, F. V. Martin; Phys. Rev. 124, 830 (1961)

L. Keszthelyi, I. Berkes, I. Demeter, I. Fodor; Nuclear Phys. 23, 513 (1961)

W. H. Kelly, G. B. Beard; Nuclear Phys. 27, 188 (1961)

H. W. Kenda11, J. I. Friedman, E. F. Erickson, D. A. M. Gramm; Phys. Rev. 124, 1596 (1961)

M. Kimura, K. Shoda, N. Mutsuro, T. Tohei, K. Sato,

K. Kuroda, K.Kuriyama, T.Akiba; Nuclear Phys. 23, 338 ( 1961)

A. P. Komar, B. A. Bochagov, G. E. Solyakin; Dokl.

Kuo Ch'i-di, B. S. Ratner, B. V. Sergeev; Zhur. Ekso.

i Teoret Fiz. 40, 85 (1961); Soviet Dhys. JETP 13,60(1961)

F. Lacoste, G. R. Bishop; Nuclear Dhys. 26, 511 (1961)

E. D. Makhnovskii;Zhur. Eksn. i Teoret. Fiz. 41, 1091

(1961); Soviet Phys. JETD 14,779 (1962)

M. Masuda; J. Phys. Soc. Japan 16, 1801 (1961)

V. Meyer, H. Muller, H. H. Staub, R. Zurmuhle; Nuclear Phys. 27, 284 (1961)

(1961)

G. Moscati; Nuclear nhys. 26, 321 (1961)

Rev. 121,630 (1961)

hys. 22, $484(1961)$

R. Nor dhagen; Nuclear Phys. 27, 112 (1961)

J. P. Perez y Jorba, H. Nguyen Ngoc; J. Phys. Radium

22, 551 (1961) 
61 Ro 1 T. Rothem, F. R. Metzger, C. P. Swann; Nuclear Phys. 22,505 (1961)

61 Ro 2 J. P. Roalsvig, I. C. Gupta, R. N. H. Haslam; Can。

J. Phys. 39, 643 (1961)

$61 \mathrm{Sa} 1$ D. Sadeh; Phys。 Rev • 123, 855 (1961)

$61 \mathrm{Sa} 2$ C. P. Sargent, W. Bertozzi, P. T. Demos, W. Turchinetz;

Proceedings of the Symposium on Neutron Time-of-Flight Methods, p. 353 (1961)

61 Se 1 R. E. Segel, J. W. Daughtry, J. W. Olness; Phys. Rev. 123, 194 (1961)

$61 \mathrm{Se} 2$ R. E. Segel, M. J. Bina; Phys. Rev. 124, 814 (1961)

61 Se 3 W. Sebaoun, H. Gauvin; J. Phys. Radium 22, 771 (1961)

61 Se 4 W. Sebaoun, J. Dhys. Radium 22, 735 (1961)

$61 \mathrm{Sh} 1$ K. Shoda, K. Kobayashi, S. Siina, K. Abe, M. Kimura;

J. Dhys. Soc. Japan 16, 1031 (1961)

61 Sh 2 V. G. Shevchenko, B. A. Yur 'ev; Zhur. Eksp. i Teoret. Fiz. 41, 1421 (1961); Soviet Phys. JETP 14, 1015 (1962)

61 Sh 3 K. Shoda; J. Dhys. Soc. Japan 16, 1841 (1961)

61 Sh 4 K. Shoda, S. Siina, K. Kobayashi, K. Abe, M. Kimura;

J. Phys. Soc. Japan 16, 1807 (1961)

$61 \mathrm{Sh} 5$ K. Shoda, K. Kobayshi, S. Siina, K. Abe, M. Kimura;

J. Dhys. Soc. Japan 16, 1031 (1961)

61 Sh 6 V. G. Shevchenko, B. A. Yur'ev; Izv. Akad Nauk SSSR 25, 1269 (1961)

$61 \mathrm{Su} 1$ M. Sugawara; J. Phys. Soc.Japan 16, 1857 (1961)

61 Sw 1 C. P. Swann, V。K. Rasmussen, F。 R. Metzger; Phys.

Rev. 121, 242 (1961)

$61 \mathrm{Ta} 1$ F. Tagliabue, J. Goldemberg, Nuclear Phys. 23, 144 (1961)

61 Th 1 I. M. Thorson, L. Katz; Proc. Phys. Soc. 77, 166 (1961)

61 To 1 T. Tohei, M. Sugawara, S. Mori, M. Kimura; J. Phys.

Soc. Japan 16, 1657 (1961)

$61 \mathrm{Va} 1$ V. J. Vanhuyse, W. C. Barber; Nuclear Phys 26, 233 (1961)

$61 \mathrm{Va} 2$ A. K. Val'ter, Yu. P. Antuf ev, E. G. Konanets, A. N.

L voy, P. Tsytko; Zhur. Eksp. i Teoret.Fiz• 41, 1499 (1061); Soviet Phys. JETP 14, 1035 (1962)

$61 \mathrm{Va} 3 \mathrm{~K}$. J. Van Oostrum, N. Hazewindus, A. H. Wapstra, J. W. Olness, J. L. Parker; Nuclear Phys. 25, 409 (1961)

61 Wa 1 A. Wataghin, R. B. Costa; A. M. Freire, J. Goldemberg; Nuovo Cimento 19,864 (1961)

61 We 1 R. E. Welsh, D. J. Donahue; Phys. Rev。 121, 880 (1961)

61 Wi 1 W. S。 C. Williams, H. S. Caplan, D. T. Stewart; Proc.

Phys. Soc. (London) 78, 1125 (1961)

61 Wo 1 J. H. Wolfe, J. P. Hummel; Phys. Rev. 123, 898 (1961)

62 An 1 E. S. Anashkina; Zhur. Eksp. i Teoret. Fiz. 43, 1197 (1962); Soviet Phys. JETP 16, 847 (1963)

62 An 2 Yu. P. Antuf'ev, A. K. Val'ter, A. N. L'vov, E. G. Kopanets, S. P. Tsytko; Zhur. Eksp. i Teoret. Fiz. 42,386 (1962); Soviet Phys. JETP 15, 268 (1962)

$62 \mathrm{Ba} 1$ V. V. Balaskov, V. N. Fetisov; Izv. Akad. Nauk

SSSR, Ser. Fiz. 26, 1188 (1962) 
62 Be 1 A. S. Belousov, S. V. Rusakov, E. I. Tamm; Zhur. Eksp. i Teoret。Fiz。 43, 813 (1962); Soviet Phys. JETP $16,576 \quad(1963)$

62 Be 2 G. Ben-David (Davis), B. Huebschmann; Phys. Letters $3,87(1962)$

62 Be 3 C. Becchi, L. Meneghetti, S。 Vitale; Phys。 Letters 3 , 89 (1962)

62 Be 4 I. Berkes, I. Dezsi, I. Fodor, L. Keszthely; Nuclear Dhys. 39, 631 (1962)

62 Bi 1 D. G. Bizzeti, A。 M. Bizzeti-Sona, M. Bocciolini,

G. DiCanoliacco, M. Mando; Nuovo Cimento 26, 1412 (1962)

$62 \mathrm{Bi} 2$ G. R。 Bishon, D。 Bo Isabelle; Phys。 Letters 1, 323 (1962)

$62 \mathrm{Bi} 3$ Go R。 Bishon, D。 Bo Isabelle; Phys. Letters 3, 74 (1962)

$62 \mathrm{Bi} 4$ G. R。 Bishon, B。 Grossetete, J. C. Risset; J. Phys. Radium 23, 31 (1962)

62 Bo 1 O. V. Bogdankevich, Bo I. Goryachev, V。 A. Zapevalov; Zhur. Eksp. i Teoret。Fiz. 42, 1502 (1962); Soviet Phys. JETP $15,1044(1962)$

62 Bo 2 L. N. Bolen, W. D. Whitehead; Phys. Rev• Letters 9, 458 (1962)

62 Bo 3 G. Bonazzola, O。A. Borello, So Costa, S. Ferroni; Nuclear Phys. 34, 637 (1962)

62 Bo 4 R. Bosch, J. Lang, R。 Muller, W. Wolfli; Phys. Letters $1,114(1962)$

62 Bo 5 O. Borello, F. Ferrero, R. Malvano, A. Molinar1; Nuclear phys. 31, 53 (1962)

62 Bo 6 E. C. Booth, K. A. Wright; Nuclear Phys. 35, 472 (1962)

62 Bo 7 R. Bosch, Jo Lang, R。 Muller, W. Wolfli; Helv. Phys. Acta $35,288(1962)$

62 Bo 8 B. A. Bochagov, A. D. Komar, Go E. Solyakin; Zhur. Eksp. i Teoret. Fiz. 43, 1611 (1962); Soviet Phys• JETP $16,1135(1963)$

$62 \mathrm{Br} 1 \mathrm{H}$. Breuer, W. Pohlit; Nuclear Phys. 30, 417 (1962)

$62 \mathrm{Br} 2 \mathrm{D}$. W. Braben, L. L. Green, J. C. Willmott; Nuclear Phys。32, 584 (1962)

$62 \mathrm{Br} 3$ E. Braun; Z. Physik 166, 62 (1962)

$62 \mathrm{Br} 4 \mathrm{H}$. Breuer; Z. Naturforsh. 17A, 584 (1962)

$62 \mathrm{Bu} 1 \mathrm{~N}$. A. Burgov, G。 V。 Danilyan, B。 S. Dolbilkin, L. E. Lazareva, F。A. Nikolaev; Zhur. Eksn. i Teoret。 Fiz。 43, 70 (1962); Soviet Phys。 JETP 16, 50 (1963)

$62 \mathrm{Ca} 1 \mathrm{~J} . \mathrm{H}$. Carver, G。 E. Coote, T. R。 Sherwood; Nuclear Phys. 37, 449 (1962)

$62 \mathrm{Ca} 2$ J. H. Carver, D。 Co Peaslee, R。 B. Taylor; Phys. Rev. 127, 2198 (1962)

$62 \mathrm{Ca} 3 \mathrm{~S}$. Cavallaro, R。 Potenza, A. Rubbino; Nuclear Phys. 36, $597(1962)$

62 Ch 1 R. E. Chrien, H。 H. Bolotin, H. Palevsky; Phys. Rev。 127,1680 (1962)

$62 \mathrm{Ch} 2$ V. Po Chizhov, A. P. Komar, L。 A. Kul'chitskil, A. V. Kulikov, E. Do Makhnovsky, Yu. M。 Volkov; Nuclear Phys. $34,562(1962)$ 
$62 \mathrm{Cl} 1$ F. M. Cliseman, A. J. Bureau, M. G. Stewart; Phys. Rev. $126,1322(1962)$

$62 \mathrm{Cu} 1$ B. Cujec; Nuclear Phys. 37, 396 (1962)

F 2 De 1 H.E.Del Bianco, W.E.Stephens; Phys. Rev. 126, 709 (1962)

62 De 2 B. I. Deutch; Nuclear 'hys. 30, 191 (1962)

62 De 3 H. G. DeCarvalho, A.Manfredini, M.Muchnik, M.Severi,

H. Bosch, J. Lang, R. Muller, W. Wolf 1 i; Nuovo Cimento 25, $534(1962)$

62 Do 1 W. R. Dodge, W. C. Barber; nhys. Rev • 127, 1746 (1962)

62 Dr 1 T. N. Dragnev, B. D. Konstantinov; Zhur. Eksp. i Teoret. Fiz. 42, 344 (1962); Soviet nhys. JETP 15, 236 (1962)

62 Ed 1 R. D. Edge, G. H. Deterson; 'hys. Rev. 128, 2750 (1962)

62 El 1 R. J. Ellison, B. Dickinson; Nuclear Phys.

$35,606(1962)$

$62 \mathrm{Em} 1$ V. Emma, C. Milone; S. Jannelli, F. Mezzanares; Nuovo Cimento $26,890(1962)$

62 Eu 1 D. Euthymiou, D. Axe1; Dhys. Rev. 128,274 (1962)

62 Fi 1 F. W. K. Firk; nhys. Rev. Letters 8, 321 (1962)

62 Fu 1 S.C.Fultz, R.I.Bramblett, J.T.Caldwell, N. E. Hansen

C. J. Jupiter; Dhys. Rev. 129, 2345 (1962)

$62 \mathrm{Fu} 2$ S. C. Fultz, R. L. Bramblett, J. T. Caldwell, N. A. Kerr; nhys. Rev. 127, 1273 (1962)

$62 \mathrm{Fu} 3$ E. G. Fuller, E. Hayward; Nuclear Dhys. 30, 613 (1962)

$62 \mathrm{Fu} 4$ F. G. Fuller, E. Hayward; Nuclear Dhys. 33, 431 (1962)

$62 \mathrm{Fu} 5$ H. Fuchs, D. Haag, K. H. Lindenberger, U. Meyer-

Berkhout; Z. Naturforsch 17a, 439 (1962)

$62 \mathrm{Fu} 6$ H. Fuchs, R. Kosiek, U. Meyer-Ber'shout; Z. Dhysik $166,590(1962)$

$62 \mathrm{Ga} 1$ C. C. Gardner, J. D. Anderson; nhys. Rev. 125, 626 (1962)

$62 \mathrm{Ge} 1$ D. S. Gemmell, G. A. Jones; Nuclear Dhys. 33, 102 ( 1962 )

62 Gl 1 D. W. M. Glaudemans, n. M. Endt; Nuclear Phys. $30,30(1962)$

62 Go 1 A. N. Gorbunov, V. A. Dubrovina, V. A. Osipova, V. S. Silaeva, D. A. Cerenkov; Zhur. Eksn. i Teoret. Fiz. 42, 747 (1962); Soviet nhys. JET? 15, 520 (1962)

62 Go 2 A. N. Gorbunov, V. A. Osipova; Zhur . Eksp. i Teoret. Fiz. 43, 40 (1962); Soviet Phys. JETP 16, 27 (1963)

62 Go 3 D. Goldman, A. F. T. Niza, E. Silva; Nuovo Cimento 25 , $41(1962)$

62 Go 4 A. N. Gorbunov, D. K. Kaipov, K. G. Kuvatov; Trudy Inst. Yadernoi Fiz., Akad. Nauk Kazakh. SSR 5, 135(1962)

$62 \mathrm{Gr} 2 \mathrm{~A}$. G. Gregory, T. R. Sherwood, E. W. Titterton; Nuclear Dhys. $32,543(1962)$

62 He 1 K. O. Hermann, J. A. Scheer; Z. Dhysik 170, 162 (1962)

$62 \mathrm{Hu} 1$ J.R.Huizenga, K.M.Clarke, J.E.Girdler; R. Vandenbosch; Nuclear nhys. 34, 439 (1962)

$62 \mathrm{Hu} 2 \mathrm{~J} . \mathrm{R}$. Huizenga, R. Vandenbosch; Nuclear Thys. 34, 457 (1962)

$62 \mathrm{Ja} 1$ H. E. Jackson; 'hys. Rev. 127, 1687 (1962)

62 Jo 1 S.A.E.Johansson, B.Forkman; Nuclear nhys. 36, 141 (1962)

62 Jo 2 W. John, J. M. Drosser; Dhys. Rev. 127, 231 (1962)

$62 \mathrm{Ka} 1$ D. K. Kaipov, Yu. K. Shubnyi, R. B. Begzhanov, A. A. Islamov, Zhur. E'sp. i Teoret. Fiz. 43, 808 (1962); Soviet Dhys. JETP 16, 572 (1963) 
H. W. Kendall, I. Talmi; Dhys. Rev. 128, 792 (1962)

A. D. Komar, A. V. Kulikov, V. D. Chizhov, I. D. Yavor, Yu. M. Volkov; Zhur. E'ssn. i Teoret. Fiz. 43, 1657 (1962); Soviet nhys. JETP 16, 1168 (1963)

62 Ko 2 A. D. Komar, Ya. Krzhemenek, I. D. Yavor; Nuclear Phys. $34,551(1962)$

62 Ko 3 K. Kotajina; Nuclear Thys. 39, 89 (1962)

62 Ko 4 A. D. Komar, B. A. Bochagov, V. I. Fadeev; Do'rl. Alsad. Nauk SSSR 146, 1051 (1962); Soviet Phys. Doklady 7, $913(1963)$

$62 \mathrm{Kr} 1$

M. Kregar, B. Dovh; Dhys. Letters 2, 103 (1962)

$62 \mathrm{Ku} 1$

K. Kuriyama; J. Ohys. Soc. Janan 17, 1681 (1962)

62 La 1

B. T. Lawergren, T. R. Ophel; Phys. Letters 2, 265 (1962)

62 Li 1 F. F. Liu, F. J. Loeffler, T. R. nalfrey, Y. S. Kim; Phys. Rev. 128, 2784 (1962)

62 Li 2 N. Lingappa, E. Kondaiah, C. Badrinathan, M. D. Deshpande,

M. Balakrishnan; Nuclear Phys. 38,146 (1962)

$62 \mathrm{Ma} 1$ I. G. Main; Nuovo Cimento 26, 884 (1962)

62 Me 1 F. R. Metzger; nhys. Rev. 128, 2332 (1962)

62 Me 2 F. R. Metzger; Dhys. Rev. 127, 220 (1962)

$62 \mathrm{Mi} 1$ J. A. Miskel, K. V. Marsh, M. Lindner, R. J. Nagle; Dhys. Rev. 128,2717 (1962)

$62 \mathrm{Mi} 2 \mathrm{~J}$. Miller, C. Schuh1, G. Tamas, C. Tzara; Dhys. Letters $2,76(1962)$

$62 \mathrm{Mi} 3$ J. Miller, C. Schuh1, C. Tzara; Nuclear Dhys. 32 , $236(1962)$

$62 \mathrm{Mi} 4$ U. Miklavzic, N. Bezic, D. Jamnik, G. Kernel, Z. Milavc, J. Snajder; Nuclear Dhys. 31, 570 (1962)

62 Mo 1 W. L. Mouton, J. P. F. Sellschop, R. J. Keddy; Dhys. Rev. 128,2745 (1962)

62 Mo 2 W. T. Morton, T. G. Walker; Jhil. Mag. 7, 741 (1962)

62 Mo 3 G. Moscati, J. Goldemberg; Dhys. Rev. 126, 1098 (1962)

$62 \mathrm{Mu} 1 \mathrm{~N}$. Mutsuro, K. Kageyama, M. Mishina, T. Nakagawa, E. Tanaka, M. Kimura; J. Dhys. Soc. Japan.17, 1672 (1962)

$62 \mathrm{Mu} 2 \mathrm{~N}$. Mutsuro, K. Kageyama, M. Mishina, E. Tanaka, M. Kimura; J. Thys. Soc. Japan 17, 1673 (1962)

$62 \mathrm{Ne} 1$ E. B. Nelson, R. R. Carlson, L. D. Schlenker; Nuclear Dhys. $31,65(1962)$

$62 \mathrm{Ne} 2$ D. R. Neher, F. W. Drosser, Jr., R. W. Krone; Nuclear Dhys. $31,231(1962)$

62 Oc 1 J. S. O'Connell, P. A. Tipler, p. Axel; Phys. Rev. 126,228 (1962)

62 Od 1 M. Odera; J. Dhys. Soc. Japan 17, 1675 (1962)

62 Op 1 T. R. Ophel, B. T. Lawergren; Nuclear Phys. 30, 215 ( 1962)

62 Op 2 T. R. Ophel, R. N. Glover, E. W. Titterton; Nuclear Dhys. $33,198(1962)$

$62 \mathrm{~Pa} 1$ V. Parikh; Nuclear Dhys. 38, 529 (1962)

62 Da 2 A. W. Dar'ser, G. G. Shute; Australian J. Thys. 15, 443(1962)

$62 \mathrm{ne} 1$ G. A. neterson, W. C. Barber; Dhys. Rev. 128,812 (1962)

62 गr 1 F. W. Mrosser, Jr., W. D. Unruh, B. W. Wildenthal;

R. W. Krone; nhys. Rev. 125, 594 (1962) 
$62 \mathrm{Ra} 1$ L. K. Rangan, G。 I. Harris, L. W. Seagondollar;

Phys。Reve127, 2180 (1962)

$62 \operatorname{Re} 1$ G. C. Reinhardt, Wo D. Whitehead; Nuclear Phys。 $30,201(1962)$

$62 \mathrm{Se} 1$ F. D. Seward; Phys. Rev. 125, 335 (1962)

$62 \mathrm{Se} 2$ W. Sebaoun; J. Phys. Radium 23, 989 (1962)

$62 \mathrm{Sh} 1$ A. K. Shardanov, V. G. Shevchenko, Zhur. Eksp。 i Teoret. Fiz。 42, 1438 (1962); Soviet Phys。 JETP 15, 996 (1962)

$62 \mathrm{Sh} 2$ V. G. Shevchenko, B. A. Yur'ev; Zhur. Eksp. i Teoret. Fiz. 42, 707 (1962); Soviet Phys. JETP 15, 492 (1962)

$62 \mathrm{Sh} 3$ V. G. Shevchenko, B. A. Yur'ev; Zhur. Eksp. i Teoret. Fiz。 43,860 (1962); Soviet Phys. JETP 16, 609 (1963)

$62 \mathrm{Sh} 4 \mathrm{~V}$. G. Shevchenko, B. A. Yur'ev; Nuclear Phys. 37, 495 (1962)

$62 \mathrm{Sh} 5$ To Ro Sherwood, W。 E。 Turchinetz; Nuclear Phys。 29, $292(1962)$

$62 \mathrm{Sh} 6$ V. G. Shevchenko, B。 A. Yur'ev; Vestn。 Mosk。 Univ॰, Ser. III, No. $3,90(1962)$

$62 \mathrm{Sh} 8 \mathrm{~K}$. Shoda, T. Ishizuka, K॰ Shimizu, M。Akashi; J。 Phys。 Soc。 Janan 17,1536 (1962)

62 Sh 9 Ko Shoda, H. Niizeki, N. Fujiwara, A。 Okiguchi, A. Watanabe, M. Midera; J. Phys. Soc. Janan 17, 1083 (1962)

$62 \mathrm{Sh} 10 \mathrm{~K}$. Shoda, T. Ishizuka, K. Shimizu, M. Akashi; J. Phys. Soc. Japan 17, 407 (1962)

$62 \mathrm{Sh} 11$ K. Shoda, K. Abe, T. Ishizuka, N. Kawamura, M. Kimura; J. Phys. Soc. Jaban 17, 735 (1962)

$62 \mathrm{Sh} 12 \mathrm{~K}$. Shoda, T. Ishizuka, N. Kawamura, K. Abe, M. Kimura; J. Phys. Soc. Janan 17, 401 (1962)

$62 \mathrm{Sm} 1$ P. J. M. Smulders, n. M" Endt; Physica 28, 1093 (1962)

62 So 1 Yu. I. Sorokin, V. G. Shevchenko, B. A. Yur'ev; Zhur. Eksp. i Teoret. Fiz。 43, 1600 (1962); Soviet Phys。 JETP $16,1127(1963)$

$62 \mathrm{Su} 1$ B. N. Sung; J. Phys. Soc. Janan 17, 1811 (1962)

$62 \mathrm{Tu} 1$ P. M. Tutakin; Zhur. Eksp. i Teoret. Fiz. 43, 1140 (1962); Soviet Phys. JETP 16, 805 (1963)

62 Vo 1 Yu. M. Volkov, L. A. Ku 1'chitskii; Zhur. Eksn. i Teoret. Fiz. 42, 108 (1962); Soviet Phys。 JETP 15, 77 (1962)

62 Vo 2 Yu. M. Volkov, A. V. Kulikov, V. D. Chizov; Zhur. Eksp. i Teoret. Fiz. 42, 61 (1962); Soviet Phys. JETP 15, $42(1962)$

62 Wa 1 E。 K. Warburton, H. O. Funsten; Phys. Rev. 128, 1810 (1962)

63 Ak 1 A. F. Akkerman, V. L. Kochetkov, V. N. Chekanov, V. V. Suvorov, A. K. Shtolts; Zhur。 Eksp. i Teoret, Fiz。 45, 1778 (1963); Soviet Phys。 JETP 18, 1218 (1964)

63 An 1 D. W. Anderson, A. Jo Bureau, B. C. Cook, J. E。 Griffin, J。 R。 McConne11, K。 H. Nybo; Phys。 Rev。 Letters $10,250(1963)$

63 An 2 Y. D。 Antoufiev, L. M. E1-Nadi, D. A. E. Darwish, O. E. Badawy, P. V. Sorokin; Nuclear Phys. 46, 1 (1963) 
63 An 3 E. S. Anashixina; Zhur. Eisso. i Teoret. Fiz. 45, 404 (1963) Soviet Ohys. JET) 18,279 (1964)

$63 \mathrm{Au} 1$ R. H. Augustson, N. N. Kaushal, W. R. Moyer, E. J. Winhold, ?. F. Yerrin; Helv. Dhys. Acta 36, 816 (1963)

$63 \mathrm{Ax} 1$ D. Axe1, K. Min, N. Stein, D. C. Sutton; Dhys. Rev. Letters $10,299(1963)$

$63 \mathrm{Ba} 1$ W. C. Barber, J. Goldemberg, G. A. Deterson, Y. Torizulsa; Nuclear Phys. 41, 461 (1963)

$63 \mathrm{Ba} 2$ G. M. Bailey, D. R. Hebbard; Nuclear Dhys. 46, $529(1963)$

$6.3 \mathrm{Ba} 3 \mathrm{M} \cdot \mathrm{V} \cdot \mathrm{Ballariny}$; Notes de Fisica 10(No. 12), 205(1963)

$63 \mathrm{Be} 1$ B. L. Berman, L. J. Koester, Jr., J. Y. Smith; Nhys. Rev. Letters 10, 527 (1963)

$63 \mathrm{Be} 2$ M. Bernheim, G. R. Bishop; Dhys. Letters 5, 270 (1963)

$63 \mathrm{Be} 3 \mathrm{M}$. Bernheim, G. R. Bishon; phys. Letters 5, 294 (1963)

$63 \mathrm{Be} 4$ W. Bertozzi, C. n. Sargent, W. Turchinetz; Dhys. Letters

$6,108(1963)$

$63 \mathrm{Be} 5 \mathrm{~J} . \mathrm{A}$. Becker, J. D. Fox; Nuclear Phys.42, 669 (1963)

$63 \mathrm{Be} 6$ G. B. Beard, w. H. Kelly; Nuclear Ohys. 43, 523 (1963)

$63 \mathrm{Be} 7$ W. Bertozzi, ?. T. Demos, S. Kowals'si, C. n. Sarrent,

W. Turchinetz, R. Fullwood, J. Russe11; Dhys. Rev. Letters $10,106(1963)$

$63 \mathrm{Be} 8 \mathrm{M}$. Bernheim, G. R. Bishop; J. de Thysique 24, 970 (1963)

$63 \mathrm{Bi} 1$ G. R. Bishop, M. Bernheim; hys. Letters 5, 140 (1963)

$63 \mathrm{BI} 2$ G. R. Bishon, C. Betourne, D. B. Isabelle; J. de Thysique 24, 973 (1963)

$63 \mathrm{Bi} 3$ G. E. Bizina, A. G. Beda, N. A. Burgov, A. V. Davydov; Zhur. Eksn. i Teoret. Fys. 45, 1408 (1963); Soviet 'hys. JET' 19, $973(1964)$

$63 \mathrm{Bl} 1$ D. Blum, D. Barreau, J. Bellicard; nhys. Letters 4, $109(1.63)$

63 B1 2 J. P. Blanc, M. Lambert, C. F. Perdrisat;

Helva Phys. Acta 36,820 (1963)

63 Bo 1 L. N. Bolen, W. D. Whitehead; Dhys. Rev. 132, 2251 ( 1963$)$

63 Bo 2 R. Bosch, J. Lang, R. Muller, W. Wolfli; Helva. Ohys. Acta $36,625(1963)$

6.3 Bo 3 n. Bounin, G. R. Bishop; J. de गhysique 24, 974 (1963)

63 Bo 4 R. Bosch, J. Lang, R. Muller, W. Wolfli; Helva. Dhys.

Acta $36,657(1963)$

63 Bo 5 O. V. Bogdankevich, B. S. Dolbilkin, L. E. Lazareva,

F. A. Nikolaev; Zhur. Essp. i Teoret. Fiz. 45, 882 (1963); Soviet Phys. JETP 18,606 (1964)

63 Bo 6 B. A. Bochagov, A. n. Komar, V. I. Fadeev; Atomnaya

Energiya 15, 191(1963); Soviet Atomic Energy 15, 891(1963)

$63 \mathrm{Br} 1 \mathrm{R}$. L. Bramblett, J. T. Caldwe 11, G. F. Auchampaugh,

S. C. Fultz; Phys. Rev. 129, 2723 (1963)

$63 \mathrm{Bu} 1 \mathrm{~N}$. A. Burgov, G. V. Danilyan, D. S. Dolbilkin, L. E. Lazareva, F. A. Nikolaev, Izv. Akad. Nauk. SSSR 27 , $866(1963)$

$63 \mathrm{Bu} 2$ W. Buss, H. Waeffler, B. Ziegler; Dhys. Letters 4, $198(1963)$

$63 \mathrm{Bu} 3 \mathrm{~N}$. A. Burgov, G. V. Danilyan, B. S. Dolbilkin, L. E. Lazareva, F. A. Nikolaev; Zhur. Eisn. i Teoret. Fiz. 45, $1693(1963)$; Soviet Phys. JETP 18, 1159 (1964) 
$63 \mathrm{Ca} 1$ J. T. Caldwell, R. R. Harvey, R. L. Bramblett, S. C. Fultz. Dhys. Letters 6, 213 (1963)

$63 \mathrm{Ch} 1$ D. R. Chagnon, D. A. Treado; Nuclear Dhys. 40, 195 (1963)

63 Co 1 S. Costa, S. Ferroni, V. Wataghin, R. Malvano, Phys.

Letters 4, $309(1963)$

63 Co 3 S. Costa, F. Ferrero, S. Ferroni, B. Minetti, C. Molino,

R. Malvano; Dhys. Letters 6, 226 (1963)

$63 \mathrm{Cv} 1$ F. Cvelbar, A. Hudoklin, M. V. Mihailovic, M. Na jzer,

V. Ramsak; Dhys. Letters 3, 364 (1963)

63 De 1 H. G. DeCarvalho, A. Manfredini, M. Muchnik, M. Severi,

R. Bosch, W. Wolfli; Nuovo Cimento 29, 463 (1963)

$63 \mathrm{Fi} 1$ P. S. Fisher, D. F. Measday, F. A. Nikolaev, A. Kalmylrov, A. B. Clegg; Nuclear hys. 45, 113 (1963)

6.3 Fi 2 E. Finckh, R. Kosiek, K. H. Lindenberger, U. Meyer-

Ber'shout, N. Nucker, K. Schlupmann; Thys. Letters 7, 271 (1963)

63 Fi 3 E. Finclkh, R. Kosiek, K. Schlummann; Naturwiss. 50 , $326(1963)$

$63 \mathrm{Fi} 4$ E. Finc'sh, R. Kosiek, K. H. Lindenberger, K. Maier, U. Meyer-Ber shout, M. Schechter, J. Zimmerer; Z. Whysik $174,337(1963)$

$63 \mathrm{Fl} 1 \mathrm{H} \cdot \mathrm{H}$. Fleischmann; Ann. 'nhysik 12,133 (1963)

$63 \mathrm{Fl} 2$ H. H. Fleischmann, F. W. Stanek; Z. Thysik 175, 172 (1963)

$63 \mathrm{Fr} 1$ D. E. Frederick. Dhys. Rev. 130, 1131 (1963)

$63 \mathrm{Fr} 2$ E. Friedland, H. R. Lemmer; Z. Dhysik 174, 507 (1963)

$63 \mathrm{Fu} 1$

$6.3 \mathrm{Fu} 2$

$6.3 \mathrm{Fu} 3$

H. Fuchs, D. Haag; Z. Thysik 171, 403 (1963)

H. Fuchs; Z. Dhysik 171, 416 (1963)

B. Furubayashi, E. Teranishi, M. Kageyama; J. Dhys. Soc. Japan 18, $1235(1963)$

$6.3 \mathrm{Ga} 1 \mathrm{~N} . \mathrm{H} \cdot$ Gale, N. Tubbs, N. Nath, A. T. G. Ferguson; Dhys. Letters 5,345 (1963)

$63 \mathrm{Ge} 1$ K. N. Geller; Nuclear Dhys. 40, 177 (1963)

$63 \mathrm{Ge} 2$

K. N. Geller; Dhys. Rev. Letters 11, 371 (1963)

63 Go 2 A. N. Gorbunov, A. T. Varfolomeev; Dhys. Letters 5, $149(1963)$

63 Go 3 H. E. Gove; Nuclear Dhys. 49, 279 (1963)

63 Go 4 J. Goldemberg, Y. Torizuka, W. C. Barber, J. D. Walecka; Nuclear Dhys. 43, 242 (1963)

63 Go 5 S. Gorodetzky, W. Benenson, D. Chevallier, D. Disdier, F. Scheibling; Thys. Letters 6, 269 (1963)

6.3 Go 8 J. Goldemberg, E. W. Hamburger, A. Szily; An. Acad. Brasil. Cienc. 35, $169(1963)$

63 Gr 1. G. M. Griffiths, M. Lal, C. D. Scarfe; Can. J. Dhys. $41,724(1963)$

$63 \mathrm{He} 1$ D. F. Hebbard, G. M. Bailey; Nuclear Dhys. 49, 666 (1963)

63 Ho 1 D. Horvat, D. Kump, B. Dovh; Nuclear Dhys. 45, 341 ( 1963$)$

63 Is 1 D. B. Isabelle, G. R. Bishop; Nuclear nhys. 45, 209 (1963)

63 Is 2 B. S. Ishkhanov, E. N. Kornienko, Yu. I. Sorokin, V. G. Shevchenko, B. A. Yur'ev; Zhur. Eksn. i Teoret. Fiz. $45,38(1963)$; Soviet Dhys. JET' 18, 29 (1964)

$63 \mathrm{Ka} 1$ D. K. Kaipov, R. B. Begzhanov, A. V. Kuz'minov, Yu. K. Shubnyi; Zhur. Eksp. i Teoret. Fiz. 44, 181 (1963); Soviet nys. JETP $17,1217(1963)$

$63 \mathrm{Ka} 2$ T. Kaminishi, C. Kojima; Jap. J. Appl. Nhys. 2, 399(1963) 
$63 \mathrm{Ka} 3$ D. K. Kainov, Yu。K. Shubnyi, Yu。 G。 Kosyak,

R. B. Begzhanov; Zhur。Eksp॰ i Teoret。Fiz。45, 443 (1963); Soviet Phys。 JETP 18, 305 (1964)

$63 \mathrm{Ke} 1 \mathrm{H}$. W. Kendall, Jo Oeser; Phys。Rev. 130, 245 (1963)

$63 \mathrm{Ki} 1$ Y. So Kim, F. F. Liu, F。 J. Loeffler, T。 R。 Palfrey Phys. Rev。129, 1362 (1963)

$63 \mathrm{Ki} 2$ M. Kimura, K. Shoda, N. Mutsuro, Mo Sugawara, K. Abe, K. Kageyama, M. Mishina, A. Ono, T. Ishizuka, S. Mori, N. Kawamura, T。 Nakagawa, E。 Tanaka; Jo Phys。 Soc。 Janan $18,477(1963)$

63 Ko 1 A. P. Komar, B. A. Bochagov, V. I. Fadeev; Dokl. Akad。 Nauk SSSR 152, 858 (1963); Soviet Phys. Doklady 8, $978(1964)$

63 Ko 2 I。 Kohno; Jo Phys. Soc. Japan 18, 1709 (1963)

$63 \mathrm{Kr} 1 \mathrm{M}$. Kregar, B. Dovh; Nuclear Phys。 43, 170 (1963)

$63 \mathrm{Ku} 1$ L。 A. Kul'chitskii, Yu. M. Volkov; Zhur. Eks 。 i Teoret. Fiz. 44, 1153 (1963); Soviet Phys. JETP 17, $780(1963)$

$63 \mathrm{Ku} 2$ L. A. Kul'chitskii, Yu. Mo Volkov, V. P. Denisov, V. I. Orgurtsov; Izv. Akad. Nauk. SSSR 27, 1412(1963)

63 La 1 M. Langevin, J. M. Loiseaux; J. de Physique 24, 1027 (1963)

63 Le 1 F。 Ho Lewis, Jr。, J。 D。 Walecka, J. Goldemberg, W。

C. Barber, Phys。 Rev. Letters 10, 493 (1963)

63 Lo 1 F. Jo Loeffler, T. R。 Palfrey, Jr。, T。O. White, Jr., Phys。 Rev。131, 1844 (1963)

63 Lo 2 R。 Lombard, Po Kossanyi, Go R。 Bishop; Jo de Physique 24,968 (1963)

63 Mc 1 J. A. McIntyre, G. Ko Tandon; Phys。 Letters 4, 117 ( 1963 )

63 Mc 2 Go Jo McCallum; Can. J. Phys. 41, 923 (1963)

63 Mc 3 W. J. McDonald, E. Buchholz, R. N. H. Haslam; Can。 J. Phys. 41, $180(1963)$

63 Me 1 F。 R. Metzger, Ho Langhoff; Phys。Rev。132, 1753 ( 1963 )

$63 \mathrm{Mi} 1$ I. V. Mitche11, R。 B. Taylor; Nuclear Phys. 44, 664 (1963)

63 Mi 2 Ko Min, Lo No Bolen, W. D. Whitehead; Phys. Rev • 132, 749 (1963)

63 Mi 3 Ko Miyano, T. Kuroyanagi, Nuclear Phys。 49, 315 (1963)

$63 \mathrm{Mi} 4$ C. Milone; Nuclear Phys。 47, 607 (1963)

63 Mi 5 O. M० M. Mitchell, K. G. McNeill; Can。 J. Phys. 41, 871 (1963)

63 Mo 1 Wo Lo Mouton, J. Po F. Sellschon, G。 Wiechers; Phys。 Rev。 129,361 (1963)

$63 \mathrm{Mu} 1$ K. M. Murray, W. Lo Bendel; Phys。Rev。132, 1134 ( 1963 )

$63 \mathrm{Mu} 2 \mathrm{~N}$. Mutsuro, Ko Kageyama, M。 Mishina, E. Tanaka, T. Aizawa, M. Kimura; J. Dhys。 Soc. Janan 18, 599 (1963)

$63 \mathrm{Ne} 1$ B. Mo Ko Nefkens; Phys. Rev. Letters 10, 55 (1963)

$63 \mathrm{Ne} 2$ B. M. K. Nefkens; Phys. Rev. Letters 10, 243 (1963)

$63 \mathrm{Ng} 1 \mathrm{H}$. Nguyen Ngoc, Mo Hors, J. Perez y Jorba; Nuclear Phys。 42, 62 ( 1963 )

$63 \mathrm{Ng} 2 \mathrm{H}$. Nguygen Ngoc, Jo Perez y Jorba; Jo de Physique 24, 965 (1963)

63 Od 1 M. Odera, N. Yamamuro, J. Phys。 Soc. Japan 18, 325 ( 1963 ) 
63 Os 1 R。 M。 Osokina; Zhur Eksp。 i Teoret。 Fiz。 44, 444 (1963); Soviet Phys。 JETP 17, 303 (1963)

63 Pe 1 R. R. Perry, B. Mainsbridge, J. Richards; Nuclear Phys。 $45,586(1963)$

63 Pe 2 K. A。 Petrzhak, R。V。 Sedletskii; Atomnaya Energiya 15, 308(1963); Soviet Atomic Energy 15, 1025(1963)

63 Pr 1 F。 Wo Prosser, Jr., R. W。 Krone, J. J. Singh; Phys。 Rev。129, 1716 (1963)

$63 \mathrm{Pr} 2$ P. R。 Pruett; Phys。 Rev。129, 2583 (1963)

63 Re 1 No Wo Reay, N. M. Hintz, Lo Lo Lee, Jro, Nuclear Phys. 44, 338 (1963)

63 Ri 1 V. Riech; Phys. Letters 6, 267 (1963)

$63 \mathrm{Ri} 2$ Do Rice-Evans; Proc. Phys. Soc. 82, 914 (1963)

$63 \mathrm{Sa} 1 \mathrm{~K}$. Sato; J. Phys。 Soc. Janan 18, 1353 (1963)

$63 \mathrm{Sc} 1 \mathrm{~J}$. A. Scheer, K. Schlummann, F。Triantafyllidis; Phys. Letters 7, 269 (1963)

63 Sc 3 H. Schmid, W。 Scholz; Z。 Physik 175, 430 (1963)

$63 \mathrm{Se} 1$ R. E. Segel, P. P. Singh, R。 G. Allas, S。 S。 Hanna; Phys。 Rev. Letters 10,345 (1963)

63 Sh 1 V。 Go Shevchenko, B. A. Yur iev, B. P. Levkin; Zhur. Eksn。 i Teoret。Fiz. 44, 808 (1963); Soviet Phys. JETP 17, 547 (1963)

$63 \mathrm{Sh} 2$ N. K. Sherman; Phys. Letters 4, 113 (1963)

$63 \mathrm{Sh} 3$ N. K. Sherman; Phys. Letters 4, 138 (1963)

$63 \mathrm{Sh} 4$ K. Shoda, B. N. Sung, N. Kawamura, M. Oyamada, K. Abe, T. Ishizuka, M. Kimura; J. Phys。 Soc. Janan $18,152(1963)$

63 Sh 5 Yu. K. Shubnyi; Zhur. Eksp. i Teoret. Fiz. 45, 460 (1963); Soviet Phys. JETP 18, 316 (1964)

63 Si 1 L. Simons, K. E. Nysten, M. Koskelin, O. Siltanen, E. Spring, G. Wendt; Phys. Letters 3, 306 (1963)

63 Si 2 L. Simons, K. E. Nysten, E. Spring, L. Kald; H. Junger, Po Holmberg, I. Forsblom; Phys。 Letters 7, 344 (1963)

$63 \mathrm{Sk} 1$ S. Jo Skorka, R。 Hubner, T.W. Retz-Schmidt, H. Wahl Nuclear Phys. 47, 417 (1963)

$63 \mathrm{Sp} 1$ E. Spring; Soc. Sci. Fennica, Commentationes Phys-Math. 28,1 (1963)

63 St 1 R. F. Stiening, E. Loh, M. Deutsch; Phys。 Rev。 Letters 10, 536 (1963)

$63 \mathrm{Su} 1$ M. Sugawara, S. Mori, A. Ono, A. Hotta, M. Kimura; J. Phys. Soc. Jaman 18, 17 (1963)

$63 \mathrm{Su} 2$ M. Suffert, G. Costa, D. Magnac-Valette; J. de Physique 24, 1029 (1963)

63 Ti 1 P. A. Tinler, D. Axel, N. Stein, D. C. Sutton; Phys. Rev. 129, 2096 (1963)

63 Va 1 V.J.Vanhuyse, G.T.Vanpraet; Nuclear Phys. 43, 344 (1963)

$63 \mathrm{Va} 2$ A. K. Val'ter, E. G. Kopanets, A. N. L vov, S。 P. Tsytko; Izv。Akad。 Nauk。SSSR 27, 232 (1963)

63 Va 3 Vo J. Vanhuyse, Go Jo Vannraet; Nuclear Phys. 45, $602(1963)$

63 Va 4 V. J. Vanhuyse, G. J. Vanpraet, K. J. Van Camn; Phys。 Letters 5, 89 (1963)

$63 \mathrm{Va} 5 \mathrm{~A} . \mathrm{K} . \mathrm{Val}$ ter, E. G. Kopanets, A. N. L'vov, A. Stegner, S. P. Tsytko; Izv。 Akad. Nauk SSSR 27, 1419(1963) 
$63 \mathrm{Ve} 1$ A. Veres; Int. J. Apol. Rad. Isotopes 14,123 (1963)

$63 \mathrm{Ve} 2$ A. Veres; Acta Dhys. Acad. Sci. Hung。16, 261-273 (1963)

63 Wa 1 Jo Bo Warren, Ko L. Erdman, L. D. Robertson, D. A.

Axen, Jo R. MacDonald; Phys。 Rev. 132, 1691 (1963)

63 Wa 2 H. D. Warren, A. P. Batson; Nuclear Phys. 48, 361 (1963)

$63 \mathrm{Ya} 1$ N. Yamamuro; Jo Phys. Soc。 Janan 18, 11 (1963)

$63 \mathrm{Ya} 2 \mathrm{M}$. Yamanouchi; J。 Phys。Soc。 Janan 18, 638 (1963)

63 Yo 1 C。 So Young, D。 Jo Donahue; Phys. Rev。 132,1724 (1963)

63 Yo 2 F。 C. Young, Jo Co Armstrong, J。 B. Marion; Nuclear

Phys. 44, 486 (1963) (Errata: Nuclear Phys. 49, 695 (1963))

$63 \mathrm{Za} 1$ Go N. Zatsepina, V。 V. Igonin, L。 E. Lazareva, A. I.

Lenestkin; Zhur。Eksp。i Teoret。Fiz。44,1787 (1963);

Soviet Phys. JETP 17, 1200 (1963)

64 A1 1 F. R. Allum, G. M. Crawley, B. M. Snicer; Nuclear Phys。 $51,177(1964)$

64 Al 2 R。 Go Allas, S。 So Hanna, Lo Meyer-Schutzmeister,

R. E. Segel; Nuclear Phys。 58, 122 (1964)

64 A1 3 R. G. Allas, S。 S。 Hanna, Lo Meyer-Schutzmeister,

R。 E。 Sege1, P。 P。 Singh, Z。 Vager; Phys。 Rev。 Letters 13, $628(1964)$

64 A1 4 F. R。 Allum, T。W. Quirk, B. M. Sbicer;

Aust. Jo Phys. 17, 420(1964)

$64 \mathrm{Am} 1$ I. Amaldi, Jr。, Go Campos Venuti, Go Cortellessa,

C. Fronterotta, A. Reale, P. Salvadori, P. Hillman;

Phys. Rev。 Letters 13, 341(1964)

64 Ar 1 Bo Arad (Huebschmann), Go Ben-David(Davis), I。 Pelah,

Y. Schlesinger; Phys。 Rev。133, B684 (1964)

64 Ar 2 H. Artus, G. Fricke, D. E。 von Stein; Z. Physik 178, 109-112(1964)

$64 \mathrm{Ba} 1$ J。 E. E. Baglin, B. M. Snicer; Nuclear Phys. 54,549( 1964)

$64 \mathrm{Ba} 2$ E. B. Bazhanov, A. P. Komar, A. V. Kulikov; Zhur. Eks刀. i Teoret. Fiz. 46, 1497 (1964); Soviet Phys. JETP 19, 1014 (1964)

64 Be 1 C. Becchi, L. Meneghetti, Mo Sanzone, S。 Vitale; Nuclear Phys. 59, 375 (1964)

64 Be 2 C. Becchi, G. E。 Manuzio, L. Meneghetti, S. Vitale; Phys. Letters $8,322(1964)$

64 Be 3 J. Bellicard, D. Barreau, D. Blum; Nuclear Phys. $60,319(1964)$

$64 \mathrm{Be} 4$ R。 B. Begzhanov, A. A. Islamov; Zhur。 Eksp。 i Teoret. Fiz. 46, $1486(1964)$; Soviet Phys。 JETP 19, 1005 (1964)

64 Be 5 B. Lo Berman, L。 J。 Koester, Jr。, J. H. Smith;

Phys。 Rev。133, B117 (1964)

64 Be 6 G。 Bo Beard; Phys。 Rev. 135, B577-580 (1964)

64 Bi 1 G. R。 Bishop, M. Bernheim, P. Kossanyi Demay; Nuclear Phys。 54, 353 (1964) 
$64 \mathrm{Bi} 2$ G. R. Bishop, D. B. Isabelle, C. Betourne; Nuclear Phys. $54,97(1964)$

$64 \mathrm{Bi} 3$ G。 R. Bishon, C. Betourne, D. B. Isabelle; Nuclear Phys. $53,366(1964)$

64 Bi 4 G。 R. Bishop, M. Bernheim; Phys。 Letters 8, 48 (1964)

64 Bl 1 J. L. Black, G. A. Jones, P. B. Treacy; Nuclear Phys。 $54,689(1964)$

64 Bl 2 J. L. Black, N. W. Tanner; Physo Letters 11, 135 (1964)

64 Bo 1 E. C. Booth, B. Chasan, K. A. Wright; Nuclear Phys. 57, $403(1964)$

64 Bo 2 R. Bosch, Jo Lang, R. Muller, W. Wolfli; Phys. Letters $8,120(1964)$

64 Bo 3 C. D. Bowman, G. F. Auchampaugh, S. C. Fultz; Phys。 Rev。 $133, \mathrm{~B} 676$ (1964)

$64 \mathrm{Br} 1$ R. L. Bramblett, J. T. Caldwell, R. R. Harvey,

S. C. Fultz; Phys. Rev. 133, B869 (1964)

64 Co 1 E. G. Corman, R. W. Jewel1, W. John, Jo E. Sherwood,

D. White; Phys. Letters 10, 116 (1964)

64 Co 2 S. Costa, F. Ferroni, S. Ferroni, C. Molino, R. Malvano; Phys. Letters 11, 324 (1964)

$64 \mathrm{Cr} 1$ H. L. Cranne 11, T. A. Griffy; Phys。 Rev. 136 ,

B15 80-1584 (1964)

64 De 1 A. DeMarco, R. Garfagnini, G. Piragino; Phys。 Letters 10, $213(1964)$

64 De 2 V. P. Denisov, A. V. Kulikov, L. A. Kul'chitskii; Zhur. Eksp. i Teoret. Fiz 46, 1488 (1964); Soviet Phys. JETP $19,1007(1964)$

64 De 3 W. Del Bianco, W. E. Stephens, J. Wiza;

Phys. Rev. 136, B418(1964)

64 Do 1 M. M. Dorosh, A. M. Parlag, V. A. Shkoda Ulyanov,

L. A. Shabalina; Zhur. Eksp. i Teoret. Fiz。 46, 1540 (1964); Soviet Phys. JETP 19, 1042 (1964)

$64 \mathrm{Du} 1$ I. I. Dushkov, B. S. Ishkhanov, I. M. Kaitonov,

B. A. Yur'ev, V. G. Shevchenko; Phys. Letters 10, 310 (1964)

64 Ej 1 H. Ejiri, Y. Nakjima, K. Horie, S。 Matsumoto, Y. Nogami; Nuclear Phys. 51, 470 (1964)

64 Er 1 F. C. Erne, C. Van der Leun; Nuclear Phys. 52, 515 (1964)

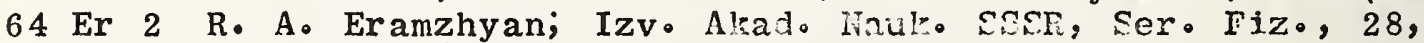
1181-1184 (1964)

$64 \mathrm{Fi} 1$ F. W. K. Firk; Nuclear Phys. 52, 437 (1964)

64 Fo 1 B. Forkman, W. Stiefler; Nuclear Phys. 56, 604 (1964)

$64 \mathrm{Fu} 1$ S. C. Fultz, R。 L. Bramblett, J. T. Caldwell,

R. R. Harvey; Phys. Rev. 133, B1149 (1964)

$64 \mathrm{Ge} 1 \mathrm{R}$. W. Gellie, K. H. Lokan; Nuclear Phys。 60, 343 (1964)

64 Gi 1 M. Giannini, P. Oliva, D. Prosperi, S. Sciuti; Nuovo Cimento 34,1116 (1964)

64 G1 1 Yu. Ya. Glazunov, M. V. Savin, I. N. Safina, E. F. Fomushkin, Yu. A. Khokhlov; Zhur. Eksp. i Teoret. Fiz. 46, 1906 (1964); Soviet Phys. JETP 19, 1284 (1964)

64 Go 1 A. N. Gorbunov, A. T. Varfolomeev; Phys. Letters 11, 137 (1964)

64 Go 2 J. Goldemberg, W. C. Barber; Phys. Rev• 134, B963 (1964)

64 Go 3 J. Goldemberg, W. C. Barber, F. H. Lewis, Jr., J. W. Walecka; Phys. Rev. 134, B1022 (1964) 
$64 \mathrm{Gr} 1 \mathrm{G} \cdot \mathrm{H} \cdot \mathrm{Greenberg}$, J० P. Roalsvig, R. N. H० Haslam;

Can. J. Phys. 42, 731-761 (1964)

$64 \mathrm{Gr} 2$ L。 Green, D。 J。 Donahue; Phys。Rev。 135, B701-705 (1964)

$64 \mathrm{Gu} 1$ F. Gudden; Phys. Letters 10, 313 (1964)

$64 \mathrm{Ha} 1$ Jo Co Hafele, F。 Wo Bingham, Jo S. Allen;

Phys. Rev。135, B365-370 (1964)

$64 \mathrm{Ha} 2 \mathrm{R}$. R。 Harvey, J。T. Caldwe1l, R。 L。 Bramblett,

S。 C. Fultz; Phys。 Rev。136, B126-131 (1964)

64 Ho 1 Mo Hors, Ho Nguyen Ngoc, Jo Perez y Jorba; Phys。 Letters 9, $40(1964)$

64 Im 1 W. L。 Imhof, F。 Jo Vaughn, L。 F。 Chase, Jr。,

H. A. Grench, M. Walt; Nuclear Phys。 59, 81 (1964)

64 Is 1 B. So Ishkanov, I。 Mo Kapitonov, V。 Go Shevchenko,

B. A. Yur'ev; Phys。 Letters 9, 162 (1964)

64 Is 2 Bo So Ishkhanov, I。 Mo Kapitonov, E。 No Kornienko, V。 G。 Shevchenko, B. A. Yur'ev; Zhur。Eksn。i Teoret。 Fiz。 46, 1486 (1964); Soviet Phys. JETP 19, 1003 (1964)

64 Ko 1 R. Kosiek, K. Maier, K. Schlummann; Phys。 Letters 9, $260(1964)$

64 Ko 2 R。 Kosiek, Ko Schlupmann, H。 W. Siebert, R。 Wendling;

Z. Physik 179, 9 (1964)

64 Ko 3 R。 Kosiek; Z。 Physik 179, 544-555 (1964)

64 Ko 4 A. Po Komar, E。 Do Ma!khnovskii; Dokl. Akad. Nauk。 SSSR 156, 774-777(1964); Soviet Phys. Doklady 9, 463-465 (1964)

64 La 1 M. Langevin, J. M. Loiseaux, J. M. Maison; Nuclear Phys. $54,114(1964)$

64 La 2 J. D. Larson, R。 Ho Spear; Nuclear Phys. 56, 197 (1964)

64 La 3 H. Langhoff; Phys。Rev 135, B1-B8 (1964)

64 La 4 H. Langhoff; Phys. Rev。136,B1590-93 (1964)

64 Li 1 F. F。 Liu; Phys。Letters 11, 306 (1964)

64 Lo 1 R。 Lombard, P。 Kossanyi Demay, G。 R. Bishon; Nuclear Phys. $59,398(1964)$

64 Lo 2 Ko H. Lokan, G. R. Hogg, P. H. Cannington, R。 J. Stewart; Phys。 Letters 11,73 (1964)

$64 \mathrm{Ma} 1$ S. Matsumoto, H. Yamashita, T。 Kamae, Y。 Nogami; Phys. Letters 12,49 (1964)

64 Me 1 F。 R。 Metzger; Phys。Rev。136, B374-379 (1964)

$64 \mathrm{Mi} 1 \mathrm{I}$. V。 Mitchel1, T。 R。 Ophel; Nuclear Phys。 58, 529 (1964)

$64 \mathrm{Mu} 1$ S。 F。 Mughabghab, W. E. Stenhens; Phys。Rev。 133, B660 (1964)

$64 \mathrm{Ne} 1$ B. M. K. Nefkens, G. Moscati; Phys. Rev。133, B17 (1964)

$64 \mathrm{~Pa} 1$ Jo C. Palathingal; Phys。 Rev。136, B1553-56 (1964)

$64 \mathrm{Ph} 1$ W. R。 Phillips; Nuclear Phys。 60,544 (1964)

$64 \mathrm{Ra} 1$ F. Rauch, E。 Rossle; Phys。 Letters 12, 217 (1964)

$64 \mathrm{Ra} 2$ B. So Ratner; Zhur。Ekso。 i Teoret。 Fiz॰ 46, 1480 (1964);

Soviet Phys. JETP 19, 1000 (1964)

$64 \mathrm{Ra} 3$ B. So Ratner; Zhur。Eksp॰ i Teoret. Fiz。 46, 1157 (1964); Soviet Phys。 JETP 19, 783 (1964)

64 Ri 1 L。 B。 Rice, L。 N. Bolen, W。 D. Whitehead; Phys。 Rev• 134, B557 (1964)

64 Sc 1 J。 A. Scheer, Ko Schlupmann, F。 Triantafyl1idis; Nuclear Phys. 56, $113(1964)$ 
64 Sc 2 Do Jo Schlueter, R。 W. Krone, F. W. Prosser, Jr.; Nuclear Phys. 58, 254 (1964)

64 Sc 3 Ko Schlupmann, R。 Wendling; Naturwissenschaften 16, 380(1964)

64 Sh 1 No K. Sherman, P. Barreau; Phys。 Letters 9, 151 (1964)

$64 \mathrm{Sh} 2 \mathrm{~A}$. Kh. Shardanov; V。 G. Shevchenko, B。 A. Yur'ev; Izv。 Alsad. Nauk SSSR 28, 60 (1964)

64 Sh 3 J. Shannon, W. E. Stephens, J. S. O'Connell; Phys. Rev • 134, B113 (1964)

64 Sh 4 Y。 M。 Shin, W。 E。 Stephens; Phys。 Rev • 136, 660-663 (1964)

$64 \mathrm{Si} 1$ L. Simons, E. Spring, Lo Kald, Ho Jungner, P. Holmberg, I. Forsblom; Acta Polytech. Scand., Phys. Nucl. Ser., No. 29, $1-19(1964)$

$64 \mathrm{Sm} 1$ Po Jo M. Smulders; Physica 30, 1197-1207 (1964)

$64 \mathrm{Sw} 1$ C. Po Swann; Phys。Rev。136, B1355-59 (1964)

$64 \mathrm{Ta} 1 \mathrm{~N}$. W。 Tanner, G。 C. Thomas, E。 D. Earle; Nuclear Phys. $52,29(1964)$

$64 \mathrm{Ta} 2$ N. W. Tanner, G. C. Thomas, E. D. Earle; Nuclear Phys. $52,45(1964)$

64 Ta 3 G. G. Taran, A。 No Gorbunov; Zhur. Eksn. i Teoret. Fiz。 46,1492 (1964); Soviet Phys。 JETP 19, 1010 (1964)

$64 \mathrm{Ta} 4$ No Wo Tanner, E. Do Earle; Phys。 Revo Letters 13,410(1964)

$64 \mathrm{Te} 1$ G. Tessler, W. E. Stenhens; Phys。 Rev。135, B129-136(1964)

64 To 1 M. E。 Toms; Nuclear Phys。 50, 561 (1964)

64 To 2 M. E. Toms; Nuclear Phys. 54, 625 (1964)

64 Tu 1 P. Mo Tutakin; Zhur. Eksp。 i Teoret。 Fiz. 46, 10 (1964); Soviet Phys. JETP 19,7 (1964)

64 Ul 1 Ho Ullrich; phys. Letters 12, 114 (1964)

64 U1 2 H. Ullrich; Z。 Physik 177, 514-528 (1964)

64 Va 1 A. K. Valiter, E. G. Konanets, A. N. L'vov, S. P. Tsytko; Izv. Akad. Nauk. SSSR, Ser. Fiz॰, 28, 271(1964)

64 Va 2 A. K. Val'ter, E. G. Kopanets, A. N. L'vov, S. P. Tsytko; Izv. Akad. Nauk。SSSR, Ser.Fiz॰, 28, 268(1964)

64 We 1 J. A. Weinman, L。 Meyer-Schutzmeister, L. L。 Lee, Jr.; Phys。Rev. 133, B590 (1964)

$64 \mathrm{Ye} 1 \mathrm{P}$. F。 Yergen, R。 H. Augustson, No No Kaushal, H. A. Medicus, W. R。 Moyer, E. J. Winhold; Phys. Rev。 Letters $12,733(1964)$ 


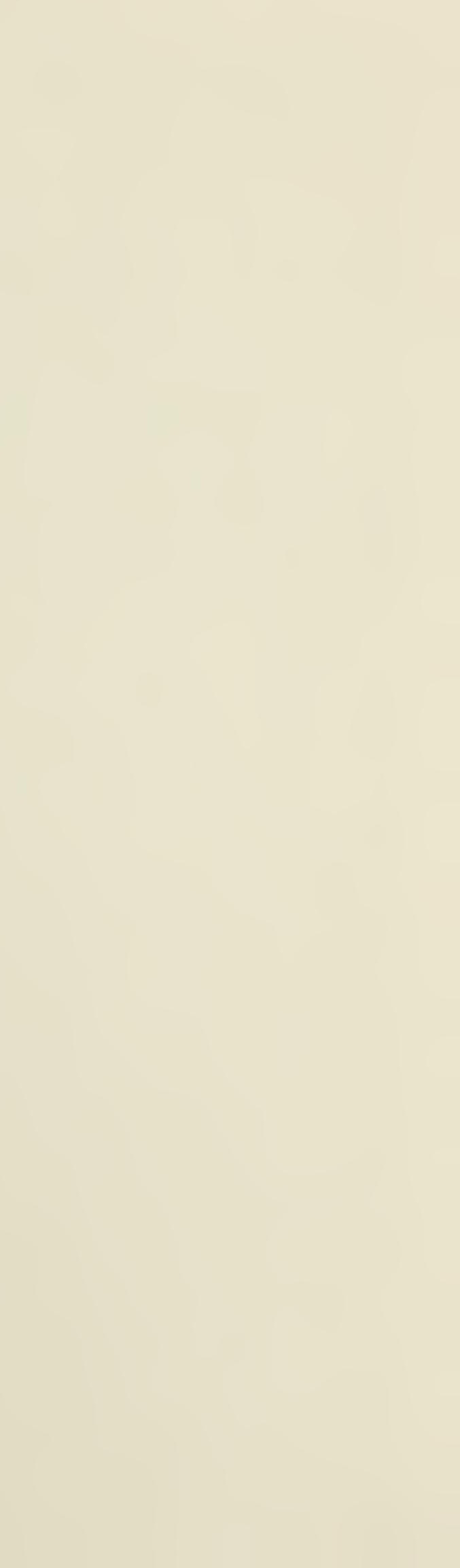
(1) 
U.S. DEPARTMENT OF COMMERCE

POSTAGE AND FEES PAID

WASHINGTON, D.C. 20230

U.S. DEPARTMENT OF COMMERCE

OFFICIAL BUSINESS 



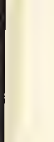

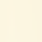





